\title{
Thermalization in one-dimensional quantum-many-body systems
}

\author{
Dissertation \\ zur Erlangung des mathematisch-naturwissenschaftlichen \\ Doktorgrades \\ „Doctor rerum naturalium" \\ der Georg-August-Universität Göttingen \\ im Promotionsprogramm ProPhys \\ der Georg-August University School of Science (GAUSS)
}

vorgelegt von

Fabian Ralf Anton Biebl

aus München

Göttingen, 2016 


\section{Betreuungsausschuss}

Prof. Dr. Stefan Kehrein

Institut für Theoretische Physik

Georg-August-Universität Göttingen

Prof. Dr. Peter E. Blöchl

Institut für Theoretische Physik

Clausthal Universität für Technologie

Prof. Dr. Markus Münzenberg

I. Physikalisches Institut

Georg-August-Universität Göttingen

\section{Mitglieder der Prüfungskommission}

Referent: Prof. Dr. Stefan Kehrein

Institut für Theoretische Physik

Georg-August-Universität Göttingen

Korreferent: Prof. Dr. Peter E. Blöchl

Institut für Theoretische Physik

Clausthal Universität für Technologie

\section{Weitere Mitglieder der Prüfungskommission}

Prof. Dr. Reiner Kree

Institut für Theoretische Physik

Georg-August-Universität Göttingen

PD Dr. Salvatore R. Manmana

Institut für Theoretische Physik

Georg-August-Universität Göttingen

Prof. Dr. Stefan Mathias

I. Physikalisches Institut

Georg-August-Universität Göttingen

Prof. Dr. Cynthia A. Volkert

Institut für Materialphysik

Georg-August-Universität Göttingen

Tag der mündlichen Prüfung: 14.12.2016 
"How peaceful life would be without love, Adso.

How safe. How tranquil.

And how dull."

Umberto Eco, The Name of the Rose 



\section{Contents}

1 Introduction 1

1.1 Motivation: Ultracold gases . . . . . . . . . . . . . . . 3

1.1.1 The scattering length ............. 4

1.1.2 Creation of ultracold gases . . . . . . . . . . . 4

1.1.3 Trapping of ultracold atoms . . . . . . . . . . . 6

1.1.4 Tuning interactions in ultracold atom systems . . . . . . 9

1.1.5 Measurement techniques . . . . . . . . . . . . 12

1.1.6 Experiments motivating the research in quantum thermalization . . . . . . . . . . . . . . 14

1.2 Time-evolution measurement of the electronic momentum distribution .......................... 18

1.2.1 Photoemission spectroscopy . . . . . . . . . . . 18

1.2.2 Angle-resolved photoemission spectroscopy . . . . . . . 21

1.2.3 Time- and angle-resolved photoemission spectroscopy . . 22

1.2.4 (Quasi) one-dimensional materials . . . . . . . . . 24

1.3 Thermalization in closed quantum systems: Theoretical overview 28

1.4 Goals and scope ..................... 33

1.4.1 The Hubbard model . . . . . . . . . . . . . . 34

1.4.2 Praseodymium calcium manganite chain: A multi-band model . . . . . . . . . . . . . . . . . 35

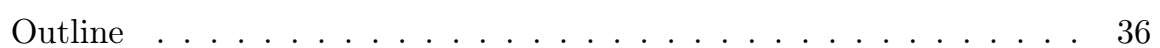

2 The multi-band Boltzmann equation $\quad 39$

2.1 Setting up a Boltzmann equation for fermions . . . . . . . . . . . 39

2.2 Linearization of the Boltzmann equation . . . . . . . . . . . . . . 44

2.3 Conserved contributions . . . . . . . . . . . . . . . 46 
2.4 State-space operators corresponding to eigenfunctions of the linearized Boltzmann operator . . . . . . . . . . . . . . 47

2.5 Interaction channels . . . . . . . . . . . . . . . . . . . 48

2.5.1 Trivial interaction channels and their elimination from the energy conservation . . . . . . . . . . . . 49

2.5.2 Existence of non-trivial interaction channels . . . . . . 50

3 Relaxation rates in the Hubbard model with next-to-nearestneighbor hopping

3.1 The Hubbard model and next-to-nearest-neighbor hopping . . . 54

3.2 The Boltzmann equation for the Hubbard model . . . . . . . . . 55

3.2.1 Linearized Boltzmann equation . . . . . . . . . . . 56

3.2.2 Separation and description of the non-trivial interaction channel ................ . . 57

3.2.3 Uniqueness of the non-trivial interaction channel for the Hubbard model . . . . . . . . . . . . . . 62

3.2.4 The reformulation of the delta distribution of the energy conservation .................. 65

3.2.5 Examples for an initial perturbation . . . . . . . . 67

3.3 Numerical scheme for diagonalizing the linearized Boltzmann equa-

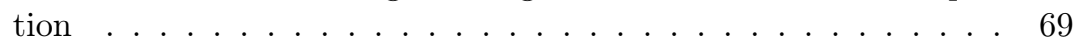

3.3.1 Calculation of the discretized linear Boltzmann operator . 69

3.3.2 Finite-size scaling . . . . . . . . . . . . 73

3.4 Results . . . . . . . . . . . . . . . . . 75

3.5 The first non-trivial eigenvalue of the linearized Boltzmann equation: The $3^{\text {rd }}$ eigenvalue . . . . . . . . . . . . . 79

3.5.1 The approximation of the $3^{\text {rd }}$ eigenfunction . . . . . . 81

3.5.2 The state-space operator corresponding to the $3^{\text {rd }}$ eigenvalue ...................... 84

3.5.3 Long-lasting currents . . . . . . . . . . . . 86

3.6 Perturbation theory of the linearized Boltzmann operator . . . . 91

3.6.1 Center of mass and relative momentum coordinates . . . 91

3.6.2 Basis transformation . . . . . . . . . . . . . 93

3.6.3 Kernel of the linearized operator in the integrable case . . 94

3.6.4 First-order perturbation theory . . . . . . . . . . . 95

3.7 Summary . . . . . . . . . . . . . . . . . 97 
4 Praseodymium calcium manganite $\quad 99$

4.1 Introduction . . . . . . . . . . . . . . . . . . . . 99

4.1.1 Crystal field splitting . . . . . . . . . . 100

4.1 .2 Competing couplings . . . . . . . . . . . . . . 104

4.1.3 Magnetic phases and one-dimensional chain . . . . . . . 107

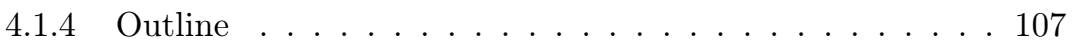

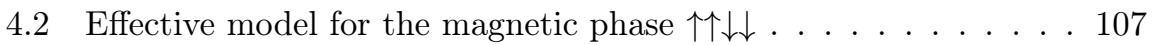

4.2.1 Effective Hamiltonian . . . . . . . . . . . . . . . . . . . 109

4.2.2 Hamiltonian in unit cell form . . . . . . . . . . . . 111

4.2.3 Hamiltonian in single-particle band form . . . . . . . . . . 113

4.3 Boltzmann equation . . . . . . . . . . . . . . 116

4.3.1 Eigenvalues and eigenfunctions of the linearized Boltzmann operator . . . . . . . . . . . . . . . . 117

4.3.2 Numerical calculation of the linearized Boltzmann operator 118

4.3.3 Corresponding state-space operators . . . . . . . . . . 120

4.3.4 Eigenfunctions with eigenvalue zero and corresponding conserved quantities . . . . . . . . . . . . . 121

4.4 Result: The lowest non-zero eigenvalue and its eigenfunction . . 122

5 Conclusions and outlook $\quad 127$

Acknowledgments/Danksagung $\quad 131$

A Appendix for the introduction 135

A.1 One-particle Green function for the grand-canonical ensemble . . 135

B Multi-band Boltzmann equation $\quad 139$

B.1 Derivation . . . . . . . . . . . . . . . . . 139

B.1.1 Quantum-mechanical time evolution ......... 139

B.1.2 Restricted quasi-freeness . . . . . . . . . . . . . . . . 142

B.1.3 Local approximation in time: A zero-interaction limit . . 145

B.2 Linearization . . . . . . . . . . . . . . . . . . 147

B.3 Scalar product, eigenfunctions, eigenvalues, and relaxation rates 148

B.4 Sommerfeld expansion of the scalar product . . . . . . . . . . . . 150

B.5 N-point interpolation . . . . . . . . . . . . . . . . 151 
C.1 Band form of the Hubbard Hamiltonian . . . . . . . . . . 157

C.2 Monotonicity of the NNN dispersion relation . . . . . . . . 158

C.3 The inverse dispersion relation and its first two derivatives . . . . 159

C.4 Small-temperature behavior of certain scalar products . . . . . 159

C.5 Particle and energy current _. . . . . . . . . . . . . . . . 162

C.6 Calculation of the total currents' expectation values . . . . . 166 C.6.1 General initial perturbation . . . . . . . . . . . 166

C.6.2 Exemplary initial perturbations . . . . . . . . . . . 169

C.7 Fixed-point Fermi-Dirac distribution . . . . . . . . . . . . . . 174

C.8 Stationary states for nearest-neighbor hopping . . . . . . . . . 174

C.9 Further simplification of $\mathcal{L}_{m n}^{0} \ldots \ldots \ldots \ldots$. . . . . . 175

C.10 Degenerate non-hermitian perturbation theory $\ldots \ldots \ldots \ldots 178$

C.11 Computing the first order correction of $\hat{\mathcal{L}} \ldots \ldots \ldots$

C.12 The relation between Fermi momentum and chemical potential . 182

C.13 The factor containing the Fermi-Dirac functions . . . . . . . 183

D Calculations for the PCMO chain $\quad 185$

D.1 Evaluation of the energy-conserving delta-function using paths . 185

D.2 First derivative of the paths of zero energy change . . . . . 187 


\title{
Chapter 1
}

\section{Introduction}

\begin{abstract}
"Thermodynamics is a funny subject. The first time you go through it, you don't understand it at all. The second time you go through it, you think you understand it, except for one or two small points. The third time you go through it, you know you don't understand it, but by that time you are so used to it, it doesn't bother you any more."
\end{abstract}

Arnold Sommerfeld, when he has been asked why he had never written a book about thermodynamics

Condensed matter systems play a key role in modern technologies. They are utterly indispensable for modern life and the development of new applications. Thus, a reliable description of such systems and their evolution in time is absolutely essential.

The physical picture of the electronic states is an electron gas, in which electrons are able to travel all over the structure. In realistic systems, the number of these electrons can be of the order of Avogadros number, i.e. $N_{\mathrm{el}} \sim 10^{23}$.

There may be a vast amount of particles involved, but the principle of their description follows the seemingly simple quantum mechanical time evolution, which is the Schrödinger equation (Schrödinger, 1926a,b,c,d)

$$
i \hbar \frac{\partial}{\partial t}|\psi\rangle=\hat{H}|\psi\rangle
$$

with the reduced Planck constant $\hbar$. This equation shows that the time evolution of the state $|\psi\rangle$ is driven by the hermitian Hamiltonian $\hat{H}$, which is the operator measuring the energy of the system. We consider a time-independent Hamiltonian. Hence the Schrödinger equation leads to the following time evolution of a state $|\psi\rangle$ :

$$
|\psi(t)\rangle=e^{-i \hat{H} t / \hbar}|\psi(t=0)\rangle
$$

The time-evolution operator $\exp (-i \hat{H} t / \hbar)$ is unitary, ${ }^{1}$ because $\hat{H}$ is hermitian. The naive way of calculating the time evolution is to diagonalize the Hamiltonian, i.e. $\hat{H}=\hat{T}^{\dagger} \hat{D} \hat{T}$, with $\hat{D}=\operatorname{diag}\left(E_{\alpha}\right)$ being a diagonal operator containing

\footnotetext{
${ }^{1}$ The time evolution operator is also unitary for time-dependent Hamiltonians.
} 
the Hamiltonian's eigenenergies $E_{\alpha}$ and $\hat{T}$ being the corresponding similarity transformation. Subsequently, the time-evolution operator is easily calculated:

$$
e^{-i \hat{H} t / \hbar}=e^{-i T^{\dagger} \hat{D} T t / \hbar}=T^{\dagger} e^{-i \operatorname{diag}\left(E_{\alpha}\right) t / \hbar} T=T^{\dagger} \operatorname{diag}\left(e^{-i \hat{E}_{\alpha} t / \hbar}\right) T .
$$

However, the core of the problem of describing a quantum many-body system lies at the point of the diagonalization of $\hat{H}$ : The dimension of the Hilbert space increases exponentially with system size. For example, a system of $N$ spins has $2^{N}$ basis-states. Evidently, a number of $10^{23}$ electrons cannot be treated by a numerical diagonalization. For very simple models, which describe the electrons of a metal, one already needs a supercomputer to perform a full diagonalization for 20 atoms with two electronic states per atom and 20 electrons (Dolfen, 2006).

This thesis is concerned with non-equilibrium quantum mechanics. Problems not only arise with the calculation of the complicated time evolution. For a rigorous description of an experimental setup, one also needs to know the initial state of the problem. However, it is not possible to determine all details of the initial state in experiments. The reasons for that are that states can not be measured, but only eigenvalues of observables, and that finding the composition of the state needs perfect reproductions of the exact states. In many cases, the initial state is simply assumed to be a thermal state with a well-defined temperature. Often, this assumption is not build on rigorous arguments. Hence, the questions we should ask are: when can an initial state be assumed to be thermal and can therefore be treated by the well-known tools of thermodynamics? In which kind of systems does thermalization occur? What happens if a system is not coupled to the environment?

An experimental way to learn more about large quantum systems are quantum simulators. These are experiments with ultracold gases that, inter alia, simulate simple and pure condensed matter systems. In contrast to real materials, they offer much more control of the model parameters. For example, in such settings, one can study the one-dimensional Hubbard model. This model is also one aim of our investigation. Therefore, the next section, Sec. 1.1, will deal with these ultracold gases and how they motivate the research in the field of non-equilibrium quantum many-body-physics. Afterwards, Sec. 1.2 will be concerned with the experimental technique called photoemission spectroscopy. We will present how it is used to measure the time-evolution of the electronic momentum distribution in solid samples. As we will show, there are also samples, in which the conduction electrons are confined to (quasi) one-dimensional structures. This is a great motivation for this work, because it deals with the long-time evolution of momentum distributions of one-dimensional systems. In any dimension, such distributions are a possible measure of thermalization in a system, which will be discussed in Sec. 1.3. This section will also introduce the theoretical framework of quantum thermalization. In addition, we will show how one can reconcile two basic concepts: (1) unitary time evolution, that leads to the fact that pure states always stay pure, and (2) the notion of thermalization, where thermal states are defined as non-pure density matrices for non-zero temperatures. The goals and scope of this thesis will be elaborated in Sec. 1.4. We will introduce the two models, which are considered in this work: a Hubbard model with nextto-nearest neighbor hopping and an effective model for a $\operatorname{Pr}_{\mathrm{x}} \mathrm{Ca}_{1-\mathrm{x}} \mathrm{MnO}_{3}$ chain. At last, we will give the outline of the thesis, where we will briefly discuss the content of the chapters. 


\subsection{Motivation: Ultracold gases}

Recent advances in the field of dilute, ultracold gases made it possible to investigate very pure, closed quantum systems (Greiner et al., 2002b; Kinoshita et al., 2006; Hofferberth et al., 2007; Bloch et al., 2008; Schreiber et al., 2015; Langen et al., 2015). The temperatures, which can be reached, are of $\mathcal{O}(10 \mathrm{pK})$ (Bloch et al., 2012), and the number of particles in such a gas is about $10^{4}$. In this section, we will show that, within these experiments, one cannot only study three-dimensional systems, but one can tune the setup in such a way that they become effectively low-dimensional for low temperatures. Furthermore, one can create one-, two-, and three-dimensional lattices, which makes it possible to simulate the electrons of condensed matter systems.

The subsections are organized as follows. First, we will introduce the concept of the scattering length in Sec. 1.1.1. This measure captures the essential features of the two-particle interaction potential for low temperatures. Sec. 1.1.2 will be about two important cooling mechanisms for creating ultracold gases. Moreover, we will elaborate on one important method for trapping atoms and on how to create lattices for the trapped atoms, see Sec. 1.1.3. Another great feature of ultracold gases setups we will consider is the possibility to tune the interaction between particles, which is explained in Sec. 1.1.4. After we will have explained two measurement techniques in Sec. 1.1.5, we will show experiments that are milestones for the field of quantum thermalization, see Sec. 1.1.6.

As a start, we clarify the technical terms "cold" and "ultracold". They emerge from scattering theory, in which one has cold and ultracold scattering processes. Scattering theory (see e.g. Gottfried and Yan (2003)) treats the interaction of two particles in their rest frame. Hence, one obtains a Schrödinger equation for the relative coordinate $\vec{r}$, which measures the distance of the particles. We assume a spherically symmetric potential and consider two identical particles. This leads to the Schrödinger equation of the relative motion (Goodman, 2009):

$$
\left[-\frac{\hbar^{2}}{2 m} \frac{d^{2}}{d r^{2}}+\frac{\hbar^{2} l(l+1)}{2 m r^{2}}+V(r)-E\right] u(r)=0,
$$

where $l$ is the quantum number of their relative angular momentum. The two atoms can only have a chance to react if their energy $E$ compensates for the centrifugal barrier $\hbar^{2} l(l+1) / 2 m r^{2}$. However, for small temperatures the relative energies of the particles are also small. Hence, the smaller the temperature, the lower the values of the angular momentum have to be. The centrifugal barrier only vanishes for $l=0$, which is known as $s$-wave scattering. The next partial waves with $l=1,2,3, \ldots$ are called $\mathrm{p}$-wave, $\mathrm{d}$-wave, $\mathrm{f}$-wave, and so on.

This leads to the defining hallmark of the cold regime of a quantum gas: Only partial waves with $l \lesssim 5$ can contribute to scattering. In the ultracold regime, all contributions with $l>0$ are negligible; only s-wave scattering can occur (Weiner, 2003; Levin et al., 2012).

It is important to note that there is no fixed border for the cold and ultracold regime, because the higher partial waves do not freeze out abruptly, but continuously. However, a quantum gas made of atoms is typically ultracold when the temperature is below $1 \mathrm{mK}$ (Jones et al., 2006). 


\subsubsection{The scattering length}

In this section, we introduce the scattering length. We will show that it is a measure for the two-particle interaction in the ultracold regime.

For distances beyond the influence of the potential, the scattered wave function of a plane wave $e^{i \vec{k} \cdot \vec{r}}$ is

$$
\psi(\vec{r}) \stackrel{\text { large } r}{\longrightarrow} e^{i \vec{k} \cdot \vec{r}}+f(k, \theta) \frac{e^{i k r}}{r},
$$

with the distance $r=|\vec{r}|$ from the scattering center and the angle $\theta$ between $\vec{r}$ and the wave vector $\vec{k}$ of the plane wave. The scattering amplitude $f(k, \theta)$ measures how much of the incoming wave $e^{i \vec{k} \cdot \vec{r}}$ is scattered into other directions. The latter are described by a spherical wave for long distances $r$, where a detector is usually placed. The scattering amplitude can be decomposed into partial wave contributions:

$$
f(k, \theta)=\sum_{l=0}^{\infty}(2 l+1) f_{l}(k) P_{l}(\cos (\theta)),
$$

where $P_{l}(x)$ are the Legendre-Polynomials.

For s-wave scattering, the scattering amplitude does not depend on the scattering angle $\theta$ any more (Basdevant and Dalibard, 2002): $f_{0}(k):=f(k, \theta)$. Furthermore, the small temperature implies that the particles have low energies. Due to the low density, the wave vector is very small for both bosons and fermions. Therefore, in the ultracold regime, the particle interaction is completely determined by the scattering amplitude in the limit $k \rightarrow 0$. This is known as the s-wave scattering length

$$
a_{\mathrm{s}}=-\lim _{k \rightarrow 0} f(k, \theta)=-\lim _{k \rightarrow 0} f_{0}(k) .
$$

It is a measure for the interaction strength, which becomes apparent when its Born-approximation is considered (Basdevant and Dalibard, 2002):

$$
a_{\mathrm{s}}=\frac{m}{2 \pi \hbar^{2}} \int d^{3} r V(r) .
$$

We will see in Sec. 1.1.4 that it is possible to change the scattering length in cold-gas experiments and therefore one can change the interaction of the atoms.

\subsubsection{Creation of ultracold gases}

In order to achieve temperatures of $\mathcal{O}(10 \mathrm{pK})$, a cascade of several cooling mechanisms is used. The available list of such mechanisms is shown in Fig. 1.1. Two very important stages are laser cooling and evaporative cooling, which make it possible to reach such low temperatures. Therefore, in this section, these two mechanisms are elaborated in more detail. There are also other cooling methods such as "optical molasses" (Dalibard and Cohen-Tannoudji, 1989; Phillips, 1998), magnetic traps (Pethick and Smith, 2008), and magneto-optical traps (Pethick and Smith, 2008). Cold gases can be trapped in a vacuum chamber with external magnetic fields or with the use of an atom chip. 


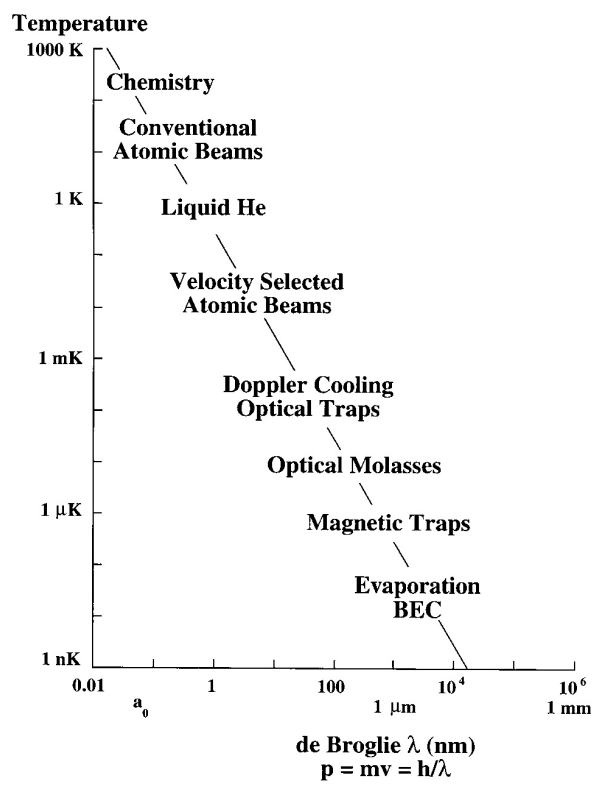

Figure 1.1: Possible cooling mechanisms. The vertical axis shows the temperatures, which can be reached with the different techniques. On the horizontal axis, there is the de Broglie wavelength for atomic sodium. Reprinted figure with permission from Weiner (2003) (C)2003 by the Cambridge University Press.

Laser cooling In principle, laser cooling achieves low temperatures by shooting photons with very small momenta onto the atom in order to slow it down. Thereby, the photon and the atom interact in the following way: The laser has a resonance frequency $\omega_{0}$, which can excite an electron. After this excitation, there is a spontaneous emission of another photon, which travels in a random direction. Hence, the momentum of the atom has changed. If the incident photons have a fixed direction, the mean acceleration of the atom is exactly in this fixed direction.

However, one wants to slow down a whole atom gas, whose constituents all travel in different directions. Thus, an atom should only interact with a photon if they travel in opposite directions. This selection is provided using the Doppler effect. It states that waves like light or sound appear to have a higher frequency when they move towards the observer, and lower if they move away from it. If both the atom and the photon move in the same direction, there should be no interaction. Therefore, the laser light has to be tuned away from the resonance frequency. If the atom and the photon travel in opposite directions, they should interact. Hence, the laser frequency has to be lowered by the Doppler shift $\omega_{\mathrm{D}}$ of the relative velocity of atom and photon, i.e. $\omega=\omega_{0}-\omega_{\mathrm{D}}$. This is referred to as red detuning of the laser with respect to the resonance $\omega_{0}$, in contrast to blue detuning, which means increasing the laser frequency.

Laser cooling can achieve very low temperatures, but has a certain temperature limit, which is known as the Doppler limit: $T_{\mathrm{D}}=\hbar \omega_{0} / 2$ (Wineland and Itano, 1979; Letokhov and Minogin, 1981; Stenholm, 1986). It is determined by the 
spontaneous emission of a photon. The kinetic energy of an initially resting atom after the emission is known as recoil energy $E_{\mathrm{r}}=\hbar^{2} k_{0}^{2} / 2 m$, where $k_{0}=\omega_{0} / c$ is the momentum of the emitted photon (Bloch et al., 2008; Stoof et al., 2009; Lewenstein et al., 2012). Even if the atom reached zero velocity by an incident photon, it would accelerate in a random direction by the spontaneous emission. Thus, the recoil energy is a measure for the Doppler temperature.

Evaporative cooling The last cooling step of the gas is inspired by hot coffee. The temperature of the coffee is mainly lowered by atoms that have so much energy that they can escape from the fluid, i.e. they evaporate. Consequently, the momentum distribution is reduced in its high-energy tail. It is important to highlight that the particles in the fluid now have to be able to interact in such a way that the momentum distribution thermalizes. This leads to a new high-energy tail, which is again cut away by evaporating atoms. Hence, for the cost of loosing a few atoms, the fluid is cooled down.

This mechanism is used to obtain ultra-cold gases. The confining potential is gradually reduced to allow fast particles to leave the gas. Obviously, the reduction has to be slower than the relaxation rate of the gas. The remaining particles are at an extremely low temperature.

The most important drawback of this process is not the loss of particles, but the fact that particles have to interact via s-wave scattering. Fermions cannot interact via s-wave scattering because of the Pauli exclusion principle. Therefore, at some point the temperature is so low that identical fermions cannot interact any more, so that the temperature cannot be further lowered any more. this problem can be solved using a two-component Fermi gas. The fermions of one species interact with the other species, which leads to thermalization.

\subsubsection{Trapping of ultracold atoms}

Now that we introduced the cooling of an atomic gas, we will show how to keep the cold gas in one place. In this chapter, we explain two techniques of trapping atoms. We elaborate on the dipole trap, which directly leads to the introduction of optical lattices for atoms.

Magnetic trap (Pethick and Smith, 2008): The principle, on which magnetic traps are based, is the Zeeman effect. Let us consider two degenerate states with magnetic moments with opposite sign. Applying a magnetic field, one level is shifted up in energy and the other one down. If the magnetic field is inhomogeneous, a particle within the state, whose level is shifted up, will move to lower magnetic fields. Therefore, this state is called a low-field seeker. The other state is called a high-field seeker. Hence, a magnetic trap will collect either low-field seekers or high-field seekers, and will push out the high-field seekers or low-field seekers, respectively.

Dipole trap (Stoof et al., 2009): Lasers can be used to trap atoms. The difference to laser cooling is that the laser is far detuned, which means that its 
frequency is far away from the resonance frequencies of the atoms. Therefore, the laser-cooling effect is negligible. The main interaction of atom and light is the following: in a semi-classical picture, the electric field of a laser, which is aligned in $x$-direction can be described by

$$
\vec{E}(\vec{r}, t)=\vec{e}_{E} \operatorname{Re}\left[E(\vec{r}) e^{i(k x-\omega t)}\right]
$$

with the position $\vec{r}=(x, y, z)$ in real-space. Let us assume that the laser is described by this electric field and that the intensity profile in $y$ - and $z$-direction is a Gaussian, i.e.,

$$
E(\vec{r})=e^{-\left(y^{2}+z^{2}\right) / 2 a^{2}(x)},
$$

with the convergence function $a(x)$, which describes the beam's focusing. Therefore, Eq. (1.9) becomes

$$
\vec{E}(\vec{r})=\vec{e}_{E} E_{0} \cos \left[(k z-\omega t) e^{-\left(y^{2}+z^{2}\right) / 2 a^{2}(x)}\right] .
$$

The light field induces a dipole in the atom: The dipole $\vec{d}$ is induced by the virtual transition of the valence electron from its unexcited state $|g\rangle$ to its excited state $|e\rangle$ :

$$
\vec{d}=e \operatorname{Re}[\langle g|\hat{\vec{R}}| e\rangle]
$$

where $\hat{\vec{R}}$ is the position operator measuring the distance of the atomic core and the atom's electron in real space. We assume that other states than $|g\rangle$ and $|e\rangle$ are not relevant. Thus, we can treat the atom as an effective two-state system.

The interaction with the electric field $\vec{E}(\vec{r})$ of the laser light is proportional to

$$
\vec{d} \cdot \vec{E}(\vec{r})
$$

In second-order perturbation theory, this leads to an effective external potential for the atom:

$$
V(\vec{r})=-\frac{1}{2 \Delta}\left|\left\langle g\left|\hat{\vec{d}} \cdot \vec{e}_{E}\right| e\right\rangle\right|^{2} E^{2}(\vec{r}) \frac{\omega_{0}}{\omega+\omega_{0}},
$$

where $\omega_{0}$ is the resonance frequency of the atom closest to the laser frequency $\omega$. The shape of this potential in real space is determined by the intensity $E^{2}(\vec{r})$. The detuning $\Delta=\omega-\omega_{0}$ can be either negative or positive, so can the effective potential. Thus, for $\Delta>0$ (blue detuning), the atom is attracted to the minima of the intensity, for $\Delta<0$ (red detuning) it is attracted to the maxima. In order to construct a trap, one focuses a blue-detuned laser at the point, where the atom gas is to be trapped. Using Eq. (1.11), the potential obtains the form

$$
V(\vec{r}, t)=-V_{0} \cos ^{2}(k x-\omega t) e^{-\left(y^{2}+z^{2}\right) / a^{2}(x)}
$$

with a constant prefactor $V_{0}$. Because the atom's reaction on the laser is much slower than $1 / \omega$, this potential is effectively averaged in time, i.e.

$$
V(\vec{r})=-\frac{V_{0}}{2} e^{-\left(y^{2}+z^{2}\right) / a^{2}(x)}
$$

A problem of this potential is that the intensity of the beam in $x$-direction does not change very much. Hence, there is only a relatively weak confinement 

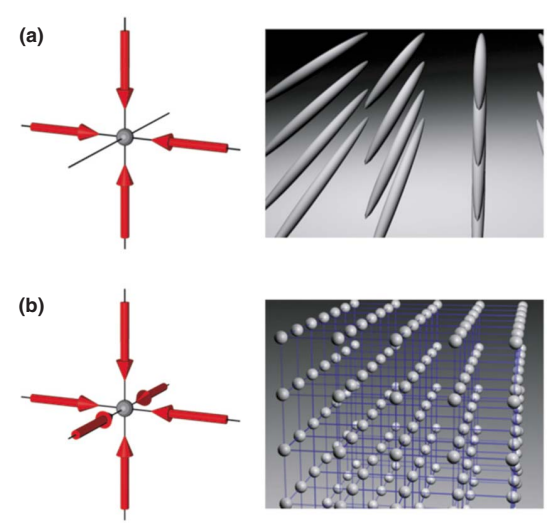

Figure 1.2: Optical lattice. Reprinted figure with permission from Bloch et al. (2008) (C) 2008 by the American Physical Society.

in $x$-direction. An improvement on this trap is the crossed-beam dipole trap Grimm et al. (2000). One uses two red-detuned lasers, which are orthogonal in space as well as in their polarization. At the crossing of the two beams, one can approximate the slowly varying convergence function by $a(x) \approx a(0)$. This leads to the potential

$$
V(\vec{r}) \approx V_{0}\left[\frac{x^{2}+y^{2}+2 z^{2}}{a^{2}(0)}-1\right] .
$$

There are many other ways to build atom traps. For example, there is also the possibility of a blue-detuned dipole trap: One surrounds the atom cloud by a wall of blue-detuned laser light, off which the atoms are repelled. The advantage is that the atom can be kept in a dark place, i.e. a place with a very low electric field Grimm et al. (2000).

Another example is the magneto-optical trap (Pethick and Smith, 2008). It combines a laser and an inhomogeneous magnetic field. The latter leads to a Zeeman shift of the electron levels of the atom. The field is tuned in such a way that the Zeeman splitting is stronger outside the trap. Hence, there, the atoms are in resonance with the laser light. This leads to a force that pulls the atoms into the trap.

Optical lattice: Detuned lasers can also be used to construct lattices for the cold atoms. Two lasers with opposite directions and same polarization lead to a standing wave by interference of the two lasers. The corresponding electric field reads

$$
\vec{E}(\vec{r}, t)=\vec{e}_{x} \operatorname{Re}\left[E(\vec{r}) e^{i(k x-\omega t)}+E(\vec{r}) e^{-i(k x-\omega t)}\right] .
$$

Assuming the same shapes $a(x)$ of the laser, as in Eq. (1.11), we obtain

$$
E(\vec{r}, t)=2 E_{0} \vec{e}_{x} \cos (k x) \cos (\omega t) e^{-\left(y^{2}+z^{2}\right) / a^{2}(x)} .
$$


The potential of the atom becomes

$$
V(\vec{r}, t)=4 V_{0} \cos ^{2}(k x) \cos ^{2}(\omega t) e^{-\left(y^{2}+z^{2}\right) / a^{2}(x)} .
$$

Here, we also have to average over time:

$$
V(\vec{r})=2 V_{0} \cos ^{2}(k x) e^{-\left(y^{2}+z^{2}\right) / a^{2}(x)} .
$$

Again, the atoms are drawn to the maxima for red-detuned lasers. They reside within the minima of the periodic factor $\cos ^{2}(k x)$. We can create a threedimensional lattice by assembling three perpendicular pairs of lasers. In the experiments we consider, the width of the laser at the crossing is much larger than the laser wavelength, i.e. $k_{j} a(0) \ll 1$ for all $j \in\{x, y, z\}$. Hence, in the region of the laser crossing, the exponential factor is approximately 1 and we obtain the time-averaged potential

$$
V(\vec{r})=V_{1} \cos ^{2}(k x)+V_{2} \cos ^{2}(k y)+V_{3} \cos ^{2}(k z) .
$$

The potential is periodic: it resembles the periodic potential of atom cores in a crystal. In this analogy, the atom gas corresponds to the valence electrons in the crystal. When the amplitudes $V_{j}$ of the optical lattice are sufficiently larger than $k_{\mathrm{B}} T$, the atoms cannot leave the optical lattice. Then, their only degree of freedom is tunneling to the neighboring potential minima, which is called hopping. Furthermore, atoms only interact if they are in the same potential well. This is described by the Hubbard model, which is one model we will study in this thesis.

By changing the intensity of one pair of counter-propagating laser beams, the potential well in that direction can be increased. Hence, the hopping-amplitude in this direction is lowered. Thus, one can tune the hopping in every direction. It is important to note, however, that the higher the intensity the more likely is a loss of two atoms on the same site due to growing inelastic collisions (Sesko et al., 1989; DePue et al., 1999). Therefore, the optical lattice depth cannot be increased to arbitrary large values.

Furthermore, one can also create other lattice forms, like a triangular lattice, the Kagome lattice and many more. This is achieved by changing the angles amongst the laser pairs.

\subsubsection{Tuning interactions in ultracold atom systems}

So far, we explained how to create and trap the cold gas. Now, a very exciting feature of cold gases experiment is presented: The possibility to tune the interaction strength of atomic interactions. We will show that this is achieved by varying an external magnetic field and making use of the Feshbach resonances (Feshbach, 1962; Fano, 1961; Stwalley, 1976; Pethick and Smith, 2008; Stoof et al., 2009).

When two atoms come close, the valence electrons interact. For our purposes, it is sufficient to consider a simple model consisting of two atoms with one valence electron. The corresponding Hamiltonian including spin-spin coupling of the 
electronic spins $\hat{\vec{s}}_{1}$ and $\hat{\vec{s}}_{2}$ and coupling to an external magnetic field $\vec{B}=B \vec{e}_{z}$ reads

$$
\hat{H}=-\frac{\hbar^{2}}{2 \bar{m}} \Delta_{\vec{r}}+V(r)+V_{\text {spin }}(r) \hat{\vec{s}}_{1} \cdot \hat{\vec{s}}_{2} / \hbar^{2}+\hat{\vec{\mu}} \cdot \vec{B} .
$$

We consider a singlet state $|S\rangle$ with electronic spins $s_{1}=s_{2}=\frac{1}{2}$, total spin $s_{S}=0$, and magnetic quantum number $m_{S}=0$ and a triplet state $|T\rangle$ with $s_{1}=s_{2}=\frac{1}{2}, s_{T}=1$, and $m_{T}=-1 . \hbar s_{j}$ and $\hbar s$ are the eigenvalues of the spin operator $\hat{\vec{s}}_{j}$, and total spin operator $\hat{\vec{s}}=\hat{\vec{s}}_{1}+\hat{\vec{s}}_{2}$, respectively. $\hbar m$ is the eigenvalue of the total-spin projection in z-direction, i.e. $\hat{s}_{z}=\hat{\vec{s}} \cdot \vec{e}_{z} \cdot|S\rangle$ and $|T\rangle$ are chosen such that they have a different magnetic moment, i.e. $\Delta \mu \neq 0$. The corresponding eigenvalues of the spin-spin couplings are

$$
\begin{aligned}
& \hat{\vec{s}}_{1} \cdot \hat{\vec{s}}_{2}|S\rangle / \hbar^{2}=\frac{1}{2}\left[s_{S}\left(s_{S}+1\right)-s_{1}\left(s_{1}+1\right)-s_{2}\left(s_{2}+1\right)\right]|S\rangle=-\frac{3}{4}|S\rangle, \\
& \hat{\vec{s}}_{1} \cdot \hat{\vec{s}}_{2}|T\rangle / \hbar^{2}=\frac{1}{2}\left[s_{T}\left(s_{T}+1\right)-s_{1}\left(s_{1}+1\right)-s_{2}\left(s_{2}+1\right)\right]|T\rangle=\frac{1}{4}|T\rangle
\end{aligned}
$$

The singlet state has the effective potential $V_{S}(r):=V(r)-\frac{3}{4} V_{\text {spin }}$ and the triplet state the effective potential $V_{T}(r):=V(r)+\frac{1}{4} V_{\text {spin }}$. We assume that $V_{T}(r \rightarrow \infty)<V_{S}(r \rightarrow \infty)$. Furthermore, the term including the global magnetic field $\vec{B}$ can be expressed by

$$
\hat{\vec{\mu}} \cdot \vec{B}=-g \mu_{B} \hat{\vec{s}} \cdot B \vec{e}_{z}=-g \mu_{B} B \hat{\vec{s}}_{z},
$$

with the gyromagnetic factor $g \approx 2$, the Bohr magneton $\mu_{B}=\hbar q / 2 m$, and the electric charge $q$. The eigenvalues of the two states are

$$
\begin{aligned}
& \hat{\vec{\mu}} \cdot \vec{B}|S\rangle=-g \mu_{B} B \hbar m_{S}|S\rangle=0, \\
& \hat{\vec{\mu}} \cdot \vec{B}|T\rangle=-g \mu_{B} B \hbar m_{T}|T\rangle=: \mu_{T} B|T\rangle .
\end{aligned}
$$

Moreover, the two states we are considering are coupled by a hyperfine interaction $V_{\mathrm{hf}}=\alpha_{\mathrm{hf}}\langle T|\hat{\vec{I}} \cdot \hat{\vec{s}}| S\rangle$, where $\hat{\vec{I}}$ is the nuclear spin operator. Moreover, we assume that the two states only couple very weakly to other eigenstates of the single atoms such that we may only consider the subsystem of the two states as a two level system. This leads to the time-independent Schrödinger equation (Stoof et al., 2009)

$$
\left[\begin{array}{cc}
-\frac{\hbar^{2} \nabla^{2}}{2 m}+V_{T}(\vec{r})+\mu_{T} B-E & V_{\mathrm{hf}} \\
V_{\mathrm{hf}} & -\frac{\hbar^{2} \nabla^{2}}{2 m}+V_{S}(\vec{r})-E
\end{array}\right]\left[\begin{array}{c}
\psi_{T}(\vec{r}) \\
\psi_{S}(\vec{r})
\end{array}\right]=0,
$$

with the spatial wave function $\psi_{X}(\vec{r})=\langle\vec{r} \mid X\rangle$ for $X \in\{S, T\}$. One eigenstate is an open channel, and the other a closed channel, i.e. the corresponding radial parts are

$$
\begin{aligned}
& u_{1}(r \rightarrow \infty)=\mathcal{A} e^{i k r}+\mathcal{B} e^{-i k r}, \text { and } \\
& u_{2}(r \rightarrow \infty)=\mathcal{C} e^{-\kappa r},
\end{aligned}
$$

respectively.

In general, there are many open and closed channels. The formalism uses a projection $\hat{P}$ onto the space of open channels and the projection $\hat{Q}=\mathbb{1}-\hat{P}$ onto the space of closed channels (Pethick and Smith, 2008). The Schrödinger 


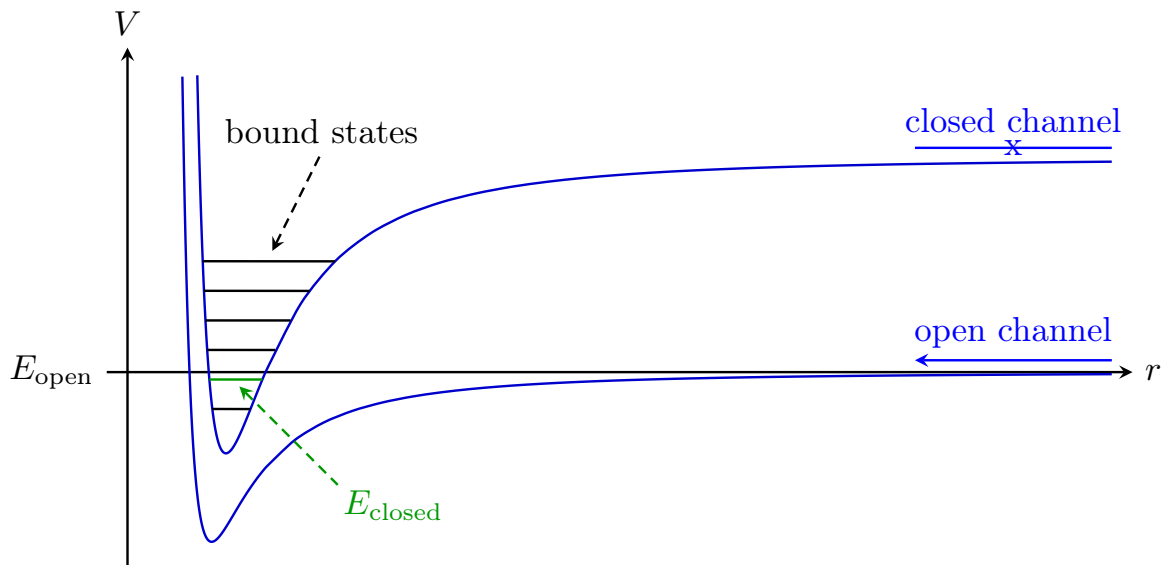

Figure 1.3: Scheme of the interaction potential of a pair of atoms satisfying the prerequisites for exhibiting Feshbach resonances. It is inspired by pictures like figure 5.4 of Pethick and Smith (2008), in which $E_{\text {open }}$ is called the threshold energy and $E_{\text {closed }}$ the resonance energy.

equation separates into two coupled equations of the two projections $\left|\psi_{P}\right\rangle=$ $\hat{P}|\psi\rangle$ and $\left|\psi_{Q}\right\rangle=\hat{Q}|\psi\rangle$ of the eigenstate $|\psi\rangle$ :

$$
\begin{aligned}
& 0=\hat{P}(\hat{H}-E)|\psi\rangle=\hat{H}_{P Q}\left|\psi_{Q}\right\rangle+\left(\hat{H}_{P P}-E\right)\left|\psi_{P}\right\rangle \\
& 0=\hat{Q}(\hat{H}-E)|\psi\rangle=\hat{H}_{Q P}\left|\psi_{P}\right\rangle+\left(\hat{H}_{Q Q}-E\right)\left|\psi_{Q}\right\rangle,
\end{aligned}
$$

with $\hat{H}_{X Y}=\hat{X} \hat{H} \hat{Y}$ and $\left|\psi_{X}\right\rangle=\hat{X}|\psi\rangle$ for all $X, Y \in\{P, Q\}^{2}$. The formal solution is

$$
\left(\hat{H}_{P P}-\hat{H}_{P Q}\left(\hat{H}_{Q Q}-E-i \delta\right)^{-1} \hat{H}_{Q P}-E\right)\left|\psi_{P}\right\rangle=0
$$

Similar to second-order perturbation theory, this leads to the following s-wave scattering length

$$
a_{\mathrm{s}}=a_{\mathrm{s}}^{(p)}+\frac{m}{4 \pi \hbar^{2}} \sum_{n} \frac{\left|\left\langle\phi_{\text {open }}\left|\hat{H}_{Q P}\right| \phi_{n}\right\rangle\right|^{2}}{E_{\text {open }}-E_{n}},
$$

neglecting the interaction between the open channels, and denoting the open channel that the system is currently in as $\phi_{\mathrm{open}}$. Moreover, $a_{\mathrm{s}}^{(p)}$ is the scattering length without closed channels and $E_{n}$ are the eigenenergies of $\hat{H}_{Q Q}$. The energy $E_{\text {open }}$ is the energy describing the following threshold: A channel is closed if the energy of the relative motion is smaller than $E_{\text {open }}$.

If there is a closed channel which has an energy $E_{\text {closed }}$ very close to $E_{\text {open }}$, the contribution of the other closed channels are negligible. Thence, the scattering length becomes

$$
a_{\mathrm{s}}=a_{\mathrm{s}}^{(0)}+\frac{m}{4 \pi \hbar^{2}} \frac{\left|\left\langle\phi_{\text {open }}\left|\hat{H}_{Q P}\right| \phi_{\text {closed }}\right\rangle\right|^{2}}{E_{\text {open }}-E_{\text {closed }}},
$$


with the non-resonant scattering length $a_{\mathrm{s}}^{(0)}$. An external magnetic field can induce shifts of the energy levels. The open channel shifts differently than the closed channel. Thus, there is a $B_{0}$, for which the denominator $E_{\text {open }}-E_{\text {closed }}$ vanishes. Therefore, we expand it in the magnetic field $B: E_{\text {open }}-E_{\text {closed }}=$ $\mu_{0}\left(B-B_{0}\right)+\mathcal{O}\left(B-B_{0}\right)^{2}$. Hence, the scattering length near $B_{0}$ has the form

$$
a_{\mathrm{s}} \approx a_{\mathrm{s}}^{(0)}\left(1-\frac{B_{1}}{B-B_{0}}\right)
$$

with a constant $B_{1}$. From scattering theory (see e.g. Sakurai (1994); Basdevant and Dalibard (2002); Friedrich (2006)), we know that the scattering length is a measure for the strength of the two-particle interaction potential. While tuning the magnetic field through a Feshbach resonance, the scattering length assumes all possible values. Therefore, in principle, one can tune the interaction to any desirable value. In an experiment, of course, the closer the magnetic field is near the resonance, the larger is the uncertainty of the scattering length. However, Feshbach resonances are a very important tool to change the effective interaction strength within a cold gas.

In summary, with the help of optical lattices, one can simulate electrons in lattice structures. One can tune the hopping of the particles from one site (potential well) to the next by altering the intensity of the lasers, which build the optical lattice. Furthermore, one exploits Feshbach resonances to change the interaction between two particles by tuning an external magnetic field. Due to the fact that the interaction of neutral atoms is short-ranged, we can approximate that atoms only interact when they reside in the same potential well. For spin- $\frac{1}{2}$ fermions this system is described by the $d$-dimensional Hubbard model

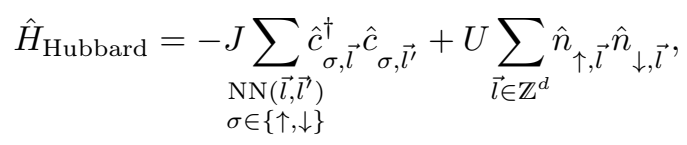

with hopping amplitude $J$, interaction $U$, the creation and annihilation operators $\hat{c}_{\sigma, l}^{\dagger}$ and $\hat{c}_{\sigma, l}$, and the number operator $\hat{n}_{\sigma, l}=\hat{c}_{\sigma, l}^{\dagger} \hat{c}_{\sigma, l}$. Moreover, the first sum runs over pairs of indices of neighboring atoms only.

\subsubsection{Measurement techniques}

In the previous sections, we explained the physics of cold gases. This section, we will be concerned with measuring the momentum distribution and the particle density in cold-gas experiments.

Time-of-flight measurement: A very common way of measuring the momentum distribution of a trapped cold gas is known as time-of-flight (TOF) measurement (Bloch et al., 2008). It is a destructive measurement, where the particles are released from the trap. After a time $t_{m}=\mathcal{O}(10 \mathrm{~ms})$, a photo of the expanded gas is taken by shining light onto the gas. The atoms absorb the light and the resulting image is captured by a camera (absorption imaging). The density distribution $n_{\text {TOF }}(\vec{x})$, which is measured by this method, is related to the momentum distribution $n_{\text {trap }}(\vec{k})$ of the previously trapped gas. Making the 


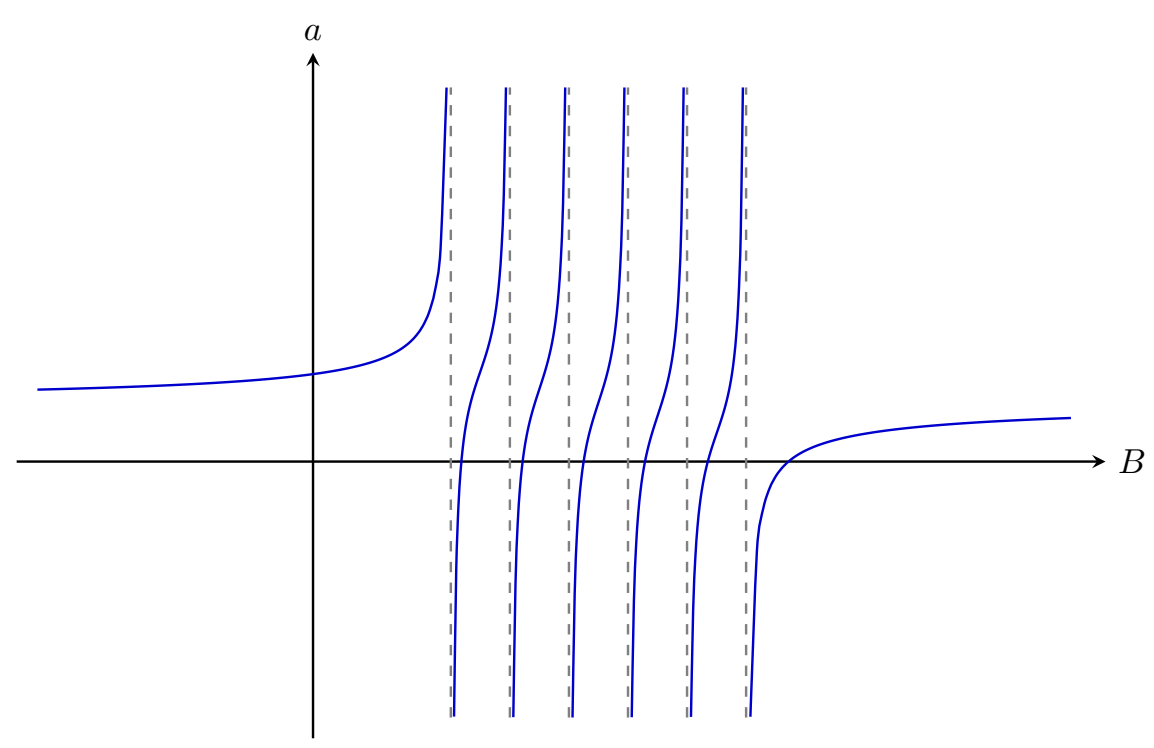

Figure 1.4: Scheme of the interaction potential of a pair of atoms satisfying the prerequisites for exhibiting Feshbach resonances.

realistic assumption of a ballistic expansion of the cloud, the relation is (Bloch et al., 2008)

$$
n_{\mathrm{TOF}}(\vec{x}(t)) \approx(M / \hbar t)^{3} n_{\text {trap }}(\vec{k}),
$$

with $\vec{x}(t)=\hbar \vec{k} t / 2 m$.

An illustrative example for an absorption image measurement is the work of Greiner et al. (2002a). They investigated a system of ultracold bosons, which is described by a Bose-Hubbard model (the bosonic equivalent of the Hubbard model Eq. (1.34)), with an additional external trap potential $\tilde{V}_{\vec{l}}$ :

$$
\hat{H}=-\underset{N \mathrm{NN}\left(\vec{l}, \vec{l}^{\prime}\right)}{ } \hat{a}_{\vec{l}}^{\dagger} \hat{a}_{\vec{l}^{\prime}}+U \sum_{\vec{l} \in \mathbb{L}} \hat{n}_{\vec{l}}\left(\hat{n}_{\vec{l}}-1\right)+\sum_{\vec{l} \in \mathbb{L}} \tilde{V}_{\vec{l}} \hat{n}_{\vec{l}},
$$

with the bosonic creation operators $\hat{a}_{\vec{l}}^{\dagger}$ and the corresponding number operator $\hat{n}_{\vec{l}}=\hat{a}_{\vec{l}}^{\dagger} \hat{a}_{\vec{l}}$. As pointed out before, the hopping amplitude $J$ is determined by the depth $V_{0}$ of the optical lattice. In this experiment, a quantum phase transition is measured when changing $V_{0}$, which can be seen by the resulting absorption images shown in Fig. 1.5. Image (a) depicts the case where the optical lattice is turned off. There, the gas exhibits Bose-Einstein condensation, which means that the single-particle ground state is occupied by an extensive fraction of the gas. Hence, picture (a) only shows a large peak at zero momentum. Turning on the lattice, more interference peaks appear at multiples of reciprocal lattice vectors $\vec{G}$, because, both, a plane wave with momentum $\vec{k}$ and one with momentum $\vec{k}+\vec{G}$ describe the same wave. These sharp peaks are hallmarks of the superfluid phase. From figure (f) to (g), the peaks vanish, because in (g) and $(\mathrm{h})$ the state is a Mott insulator. This means that the potential wells are so large that particles do not move for sufficiently low temperatures. In this case, 

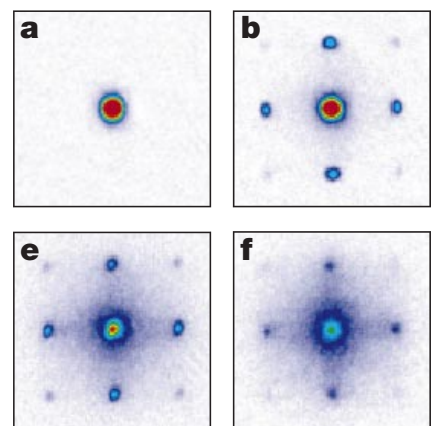
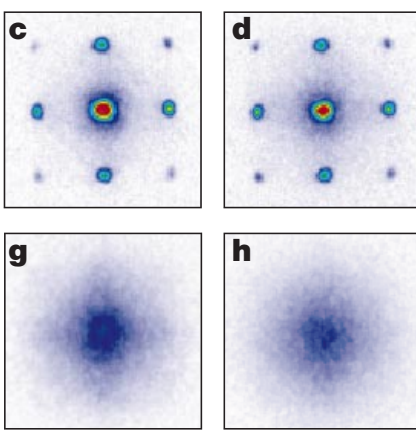

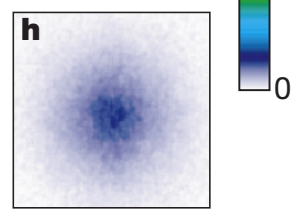

Figure 1.5: The figure shows absorption images for different optical lattice depths $V_{0}: 0 E_{\mathrm{r}}(\mathrm{a}), 3 E_{\mathrm{r}}(\mathrm{b}), 7 E_{\mathrm{r}}$ (c), $10 E_{\mathrm{r}}$ (d), $13 E_{\mathrm{r}}(\mathrm{e}), 14 E_{\mathrm{r}}$ (f), $16 E_{\mathrm{r}}(\mathrm{g}), 20 E_{\mathrm{r}}$ (h). The color scale is a measure for the atomic density. This series of pictures shows the transition from the superfluid (a-f) to the Mott-insulating phase $(\mathrm{g}, \mathrm{h})$. Reprinted by permission from Macmillan Publishers Ltd: Nature (Greiner et al., 2002a), (c)2002.

the density has sharp peaks. Therefore, the momentum distributions $(\mathrm{g})$ and (h) are broad and show no peaks.

Fluorescence imaging: Another technique is able to measure the particle density in the trap. It is a high-resolution measurement of the occupation of an optical lattice called fluorescence imaging (Bloch et al., 2012). Light with a frequency near a resonance is shone onto the optical lattice and leads to fluorescence of the atoms. To avoid hopping of the atoms, the lattice depth is increased by a factor of 100 . This, however, requires increasing the intensity, which amplifies the previously mentioned loss effect: If a lattice site is occupied by more than one atom, particles are lost in pairs due to light-induced collisions (Sesko et al., 1989). Hence, fluorescence imaging is destructive and can only measure the lattice occupation modulo 2, i.e. the parity.

\subsubsection{Experiments motivating the research in quantum thermalization}

In the previous chapters, we laid the ground work for understanding cold-gas experiments. Since this thesis deals with thermalization, we will now point out two cold-gas experiments, which had a great impact on the field of nonequilibrium physics.

Collapse and revival of a Bose-Einstein condensate: In the experiment of Greiner et al. (2002b), a Bose-Einstein condensate is in an optical lattice with a lattice depth $8 E_{\mathrm{r}}$. The optical lattice $\mathbb{L}$ is suddenly changed to a relatively high lattice depth of $22 E_{\mathrm{r}}$. In theory, this is described by a change of the Hamiltonian, which is called a (quantum) quench. The new Hamiltonian has different eigenstates. Hence, it is practically impossible that the state is an energyeigenstate or a thermal state, which means that this is an out-of-equilibrium 


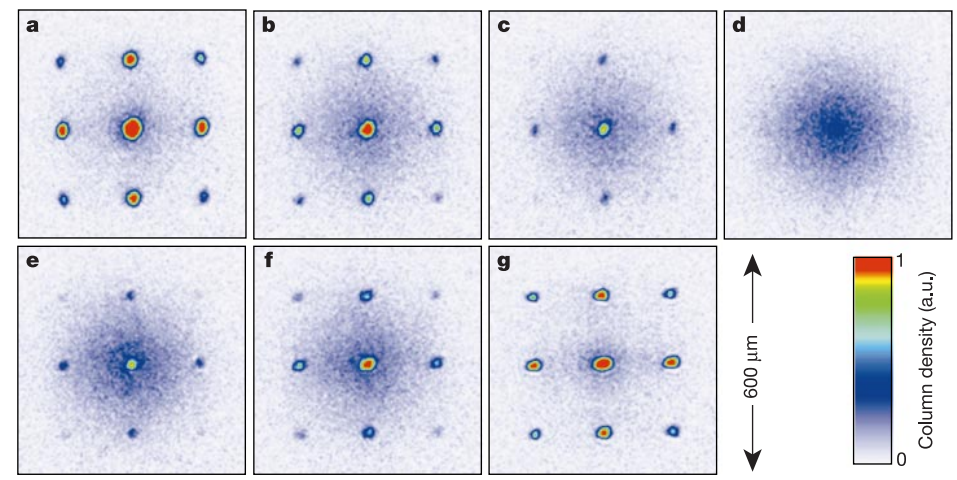

Figure 1.6: Momentum distribution of a Bose-gas observed by time of flight measurements at different times (Greiner et al., 2002b). This series shows the momentum distribution during one cycle of the collapse and revival of the Bose gas introduced in the text. Comparing these pictures to the ones in Fig. 1.5, one can see that the momentum distribution is the one of a superfluid in the beginning. Then, it changes in the one of a Mott-insulator, and cycles back to a distribution very close to the initial one. Reprinted by permission from Macmillan Publishers Ltd: Nature (Greiner et al., 2002b), (c)2002.

situation. Due to the large depth of the optical lattice, hopping is suppressed. The time evolution is solely determined by the interaction Hamiltonian

$$
\hat{H}_{\mathrm{int}}=U \sum_{\vec{l} \in \mathbb{L}} \hat{n}_{\vec{l}}\left(\hat{n}_{\vec{l}}-1\right)
$$

This leads to what is known as collapse-and-revival behavior. The reason for this can be explained as follows: The time evolution is

$$
\exp \left(-i \hat{H}_{\mathrm{int}} t / \hbar\right)=\sum_{\vec{l} \in \mathbb{L}} \sum_{n_{\vec{l}}=0}^{\infty} e^{-i U n_{\vec{l}}\left(n_{\vec{l}}-1\right) t / \hbar}\left|\vec{l}, n_{\vec{l}}\right\rangle\left\langle\vec{l}, n_{\vec{l}}\right|
$$

with the number of particles in the potential well labeled by $\vec{l}$ and the eigenstates $\left|\left\{n_{\vec{l}}\right\} \vec{l}\right\rangle$ of the corresponding number operator $\hat{n}_{\vec{l}}$. The state is assumed to be generic and therefore a superposition of all the eigenstates $\left|\vec{l}, n_{\vec{l}}\right\rangle$. However complicated this superposition may be, at times $t \in(2 \pi \hbar / U) \mathbb{Z}$ all the phase-factors $\exp \left[-i U n_{\vec{l}}\left(n_{\vec{l}}-1\right) t / \hbar\right]$ are equal to 1 , and the original state is recovered ("revival"). The momentum distribution is measured by a time-of-flight measurement, see Fig. 1.6. It oscillates back and forth from a momentum distribution of a superfluid to one of a Mott-insulator. Thus, this experiment is an example for time evolution in three dimensions without a thermalizing behavior.

Quantum Newton's Cradle: This thesis is concerned with thermalization of quantum many-body systems. A famous experiment that motivated this field of research is the Quantum Newton's Cradle by Kinoshita et al. (2006). In this experiment, a system containing cold hard-core Bosons is investigated. They are cooled down to very low temperatures and trapped in a combination of a crossed 


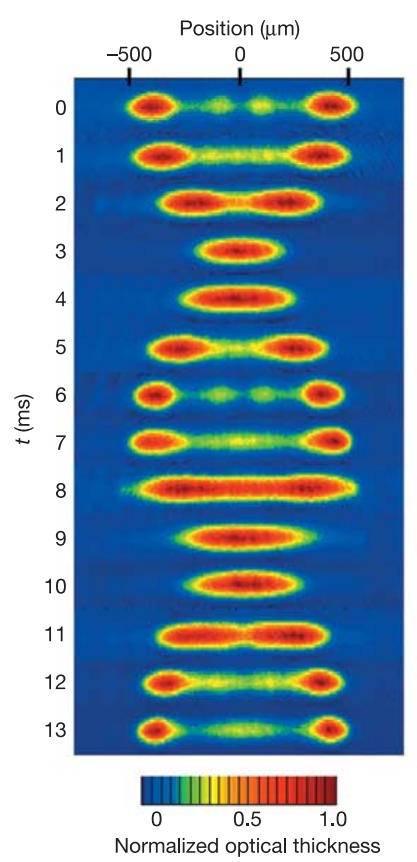

Figure 1.7: Quantum newtons cradle. This plot depicts the momentum distribution of two counterpropagating Bose-gases confined in one dimension. These measurements depict the momentum distribution. It oscillates and does not show signs of thermalization. Reprinted by permission from Macmillan Publishers Ltd: Nature (Kinoshita et al., 2006), (c)2006.

dipole trap and an optical lattice. The latter confines the particles very strongly within two dimensions, while the confinement within the third dimension is less strong. Therefore, the experimental setup consists of many parallel onedimensional tubes like the ones in Fig. 1.2a. Hence, in one run of the experiment, thousands of parallel one-dimensional Bose gases can be sampled. In one tube, there are hundreds of atoms. These atoms are prepared in a state where half of them have momentum $2 \hbar k$ and the other half $-2 \hbar k$, with the wave vector $k$ of the underlying 1d lattice. Thence, initially there are two clouds of Bosons moving in opposite directions. The time evolution of the momentum distribution is captured by time-of-flight measurements, which are plotted in Fig. 1.7. One can see that the momentum distribution oscillates. It does not show signs of thermalization. An oscillating momentum distribution implicates an oscillating particle density. The clouds oscillate back and forth with thousands of particle collisions. Despite a vast number of collisions thermalization is not seen here. There are relaxation processes, but they are negligible. They can be attributed to heating, losses from the trap, and dephasing due to trap anharmonicities.

Moreover, an important aspect of this experiment is the role of the dimensionality. Fig. 1.7 shows that the non-thermalizing behavior is present in the onedimensional version of their system. Thermalization in the three-dimensional equivalent is accomplished after 2.7 collisions per atom. However, in the one- 
dimensional case, they find that the systems are not thermalized after 600 or more collisions per atom. This is due to the fact that the underlying model is integrable in one dimension only.

The experiments discussed in this section suggest that in many-body physics the time evolution does not necessarily lead to thermalization. So far, however, we have not clarified yet what thermalization in the realm of quantum manybody physics means and entails. Thus, in the next section, we will address this issue starting with the basic theoretical frameworks of quantum mechanics and thermodynamics. 


\subsection{Time-evolution measurement of the electronic momentum distribution}

The objective of this work is to investigate the thermalization behavior of electrons in a valence band. A way of considering this is measuring the electronic momentum distribution $n^{(\mathrm{el})}(k, t)$. Therefore, this section is dedicated to an experimental technique that is able to measure the time evolution of this distribution in solid samples.

A solid consists of nuclei and electrons. Due to their interaction, they constitute a complex quantum many-body system. Photoemission spectroscopy (PES) techniques aim at gaining information about electronic occupation in a solid by shining light on them and measuring the properties of electrons that are emitted from the solid into ultra-high vacuum. This is based on the photo-electric effect found by Hertz (1887) and explained by Einstein (1905). In Sec. 1.2.1, we present a short theoretical introduction to PES.

The angle-resolved PES (ARPES) measures the energy and the angle of the emitted electrons, and therefore grants access to the spectral function, which contains all the information about the momentum distribution. In Sec. 1.2.2, we shortly explain ARPES and discuss an example for such an experiment. One can also measure the time dependence of the electronic occupation, which is known as time-resolved ARPES (trARPES). An example for this technique is shown in Sec. 1.2.3. Finally, in Sec. 1.2.4, we will show the large number of trARPES measurements treating (quasi) one-dimensional systems, which is of special interest for this thesis.

\subsubsection{Photoemission spectroscopy}

In this section, we will derive an approximate form of the electron emission rate measured by PES. We follow the derivation of Gunnarsson and Schönhammer (1987), pages 110-113. In this kind of experiment, one excites an electron into a state with such a high energy, that it is able to leave the sample. In order to describe such processes within a quantum many-body formulation, we make use of Fermi's Golden rule, see e.g. Gottfried and Yan (2003). It gives the transmission rate $\Gamma_{i \rightarrow f}$ from an initial state $|i\rangle$ to a final state $|f\rangle$ by the perturbation $\hat{V}$.

$$
\Gamma_{i \rightarrow f}=\frac{2 \pi}{\hbar}|\langle i|\hat{V}| f\rangle|^{2} \delta\left(E_{f}-E_{i}\right),
$$

where $E_{i}$ and $E_{f}$ are the initial and final energy, respectively.

Next, we approximate the photo excitations by the operator

$$
\hat{\tau}=\sum_{k k^{\prime}} \tau_{k k^{\prime}} \hat{c}_{k}^{\dagger} \hat{c}_{k^{\prime}}
$$

where $\tau_{k k^{\prime}}$ are the dipole matrix elements of an electron excitation from the single particle state $\hat{c}_{k^{\prime}}^{\dagger}|0\rangle$ to $\hat{c}_{k}^{\dagger}|0\rangle$.

Now we apply the sudden approximation. It assumes that the excited electron has no interaction with the electrons that remain in the sample. This means 
that the final state can be written like $|f\rangle=\hat{c}_{k}^{\dagger}|n\rangle$ and that its energy is $E_{f}=$ $E_{n}+\epsilon_{k}-\hbar \omega$. Hereby, $\epsilon_{k}$ is the kinetic energy of the electron created by $\hat{c}_{k}^{\dagger}$, and $\omega$ is the photon frequency. The higher $\epsilon_{k}$, the better the approximation, and this kinetic energy is typically in the range of 50 to $1000 \mathrm{eV}$. Using the sudden approximation, the matrix elements of the perturbation become

$$
\langle i|\hat{V}| f\rangle=\left\langle i\left|\hat{\tau} \hat{c}_{k}^{\dagger}\right| n\right\rangle
$$

and the energy conservation reads

$$
\delta\left(E_{f}-E_{i}\right)=\delta\left(E_{n}+\epsilon_{k}-\hbar \omega-E_{i}\right) .
$$

Furthermore, in a photoemission experiment all final states are allowed. Hence, we sum over $n$ and obtain

$$
\Gamma_{i}(k)=\sum_{n}\left|\left\langle i\left|\hat{\tau} \hat{c}_{k}^{\dagger}\right| n\right\rangle\right|^{2} \delta\left(\epsilon_{k}-\hbar \omega+E_{n}-E_{i}\right) .
$$

Our initial state is a low-temperature thermal density-matrix $\hat{\rho}=\exp (-\beta \hat{\mathcal{H}}) / Z$ describing the grand-canonical ensemble with $\hat{\mathcal{H}}=\hat{H}-\mu \hat{N}$ and the grandcanonical partition function $Z=\operatorname{tr}[\exp (-\beta \hat{\mathcal{H}})]$. Here, $\hat{H}$ and $\hat{N}$ are the Hamiltonian and total number of particles of the solid sample without the photonic perturbation $\hat{\tau}$, respectively. We assume that the time evolution of the solid sample (without the perturbation $\hat{\tau}$ by the photons) preserves the number of electrons, i.e. $[\hat{H}, \hat{N}]=0$. Hence, the eigenvalues of $\hat{\mathcal{H}}$ can be written as $K_{n}:=E_{n}-\mu N_{n}$, which is a combination of the eigenenergies $E_{n}$ and the eigenvalues $N_{n}$ of the number operator $\hat{N}$. Thence, the spectral decomposition of the density matrix is

$$
\hat{\rho}=\frac{1}{Z} \sum_{i} e^{-\beta K_{i}}|i\rangle\langle i| .
$$

Every part $\exp \left(-\beta K_{n}\right)|n\rangle\langle n|$ in the mixture leads to a certain amount of emitted electrons. Therefore, we need to sum up the individual emission rates $\Gamma_{i}(k)$ weighted by $\exp \left(-\beta K_{i}\right) / Z$ :

$$
\begin{aligned}
\Gamma(k) & =\frac{1}{Z} \sum_{i} e^{-\beta K_{i}} \Gamma_{i}(k) \\
& =\frac{1}{Z} \sum_{i, n} e^{-\beta K_{i}}\left|\left\langle i\left|\hat{\tau} \hat{c}_{k}^{\dagger}\right| n\right\rangle\right|^{2} \delta\left(\epsilon_{k}-\hbar \omega+E_{n}-E_{i}\right) .
\end{aligned}
$$

Moreover, we can express the energy difference $E_{n}-E_{i}$ using the eigenvalues $K_{n}$ :

$$
E_{n}-E_{i}=K_{n}-K_{i}+\mu\left(N_{n}-N_{i}\right)
$$

The detector measures the kinetic energy $\epsilon_{k}$ of any emitted electron with momentum $k$. Hence, PES has access to

$$
\begin{aligned}
\Gamma(\epsilon) & =\sum_{k} \delta\left(\epsilon-\epsilon_{k}\right) \Gamma(k) \\
& =\frac{1}{Z} \sum_{k} \delta\left(\epsilon-\epsilon_{k}\right) \sum_{i, n} e^{-\beta K_{i}}\left|\left\langle i\left|\hat{\tau} \hat{c}_{k}^{\dagger}\right| n\right\rangle\right|^{2} \delta\left(\epsilon_{k}-\hbar \omega+E_{n}-E_{i}\right) .
\end{aligned}
$$


We simplify the matrix element $\left\langle i\left|\hat{\tau} \hat{c}_{k}^{\dagger}\right| n\right\rangle$ by assuming that the states $|i\rangle$ contain negligible contributions from the high-lying scattering states $\hat{c}_{k}^{\dagger}|0\rangle$ that have sufficient kinetic energy $\epsilon_{k} \gtrsim 50 \mathrm{eV}$. Hence, we approximate

$$
\hat{c}_{k}|i\rangle \approx 0 \text {. }
$$

This leads to

$$
\begin{aligned}
\left\langle n\left|\hat{c}_{k} \hat{\tau}\right| i\right\rangle & =\sum_{q q^{\prime}} \tau_{q q^{\prime}}\left\langle n\left|\hat{c}_{k} \hat{c}_{q}^{\dagger} \hat{c}_{q^{\prime}}\right| i\right\rangle=\sum_{q q^{\prime}} \tau_{q q^{\prime}}\left\langle n\left|\left(\delta_{k q}-\hat{c}_{q}^{\dagger} \hat{c}_{k}\right) \hat{c}_{q^{\prime}}\right| i\right\rangle \\
& =\sum_{q} \tau_{k q}\left\langle n\left|\hat{c}_{q}\right| i\right\rangle .
\end{aligned}
$$

Furthermore we assume that the interference of different $q$ is negligible. Then, the emission rate becomes

$$
\Gamma(\epsilon)=\frac{1}{Z} \sum_{q} \sum_{i, n} e^{-\beta K_{i}}\left|\left\langle i\left|\hat{c}_{q}^{\dagger}\right| n\right\rangle\right|^{2} \delta\left(\epsilon_{k}-\hbar \omega+E_{n}-E_{i}\right) \sum_{k}\left|\tau_{k q}\right|^{2} \delta\left(\epsilon-\epsilon_{k}\right) .
$$

We approximate the factor $\left|\tau_{k q}\right|$ by an effective dipole matrix element $\tau_{\text {eff }}$ (Hedin and Lundqvist, 1970). In addition, we define $\tau(\epsilon):=\sqrt{\sum_{k} \tau_{\text {eff }}^{2} \delta\left(\epsilon-\epsilon_{k}\right)}$. Then we can write

$$
\Gamma(\epsilon)=\frac{\tau^{2}(\epsilon)}{Z} \sum_{q} \sum_{i, n} e^{-\beta K_{i}}\left|\left\langle i\left|\hat{c}_{q}^{\dagger}\right| n\right\rangle\right|^{2} \delta\left(\epsilon_{k}-\hbar \omega+E_{n}-E_{i}\right) .
$$

We can identify the Lehmann representation of the lesser Green's function $G^{<}(\vec{k}, \epsilon-\hbar \omega)$, see Eq. (A.7). It is equal to a combination of the spectral function of the retarded Green's function multiplied and the Fermi-Dirac function $f(\epsilon)=1 /[1+\exp (\beta(\epsilon-\mu))]$, see Eq. (A.13). Thence, we obtain the PES emission rate

$$
\Gamma(\epsilon)=\tau^{2}(\epsilon) \sum_{q} f(\epsilon-\hbar \omega) A(\vec{q}, \epsilon-\hbar \omega) .
$$

As a side remark, for the inverse of the PES, which is the bremsstrahlung isochromat spectroscopy (BIS), one gets a similar result, see Gunnarsson and Schönhammer (1987). The difference is that the lesser Green's function $G^{<}$is replaced by the greater Green's function $G^{>}$(definition see Eq. (A.1)). As a result, the Fermi function $f$ in Eq. (1.52) is replaced by $1-f$.

With both methods, PES and BIS, one gets access to the spectral function $A(\vec{k}, \epsilon)$. This is very valuable knowledge about the investigated sample. The spectral function not only grants access to the density of states

$$
\rho(\epsilon)=\sum_{k} A(\vec{k}, \epsilon-\hbar \omega),
$$

and the occupied density of state

$$
\sum_{k} f(\epsilon) A(\vec{k}, \epsilon-\hbar \omega)
$$

In Eq. (A.14) we show that it also grants access to the momentum distribution

$$
n(\vec{k})=\int d \epsilon G^{<}(\vec{k}, \epsilon)=\int d \epsilon f(\epsilon) A(\vec{k}, \epsilon) .
$$




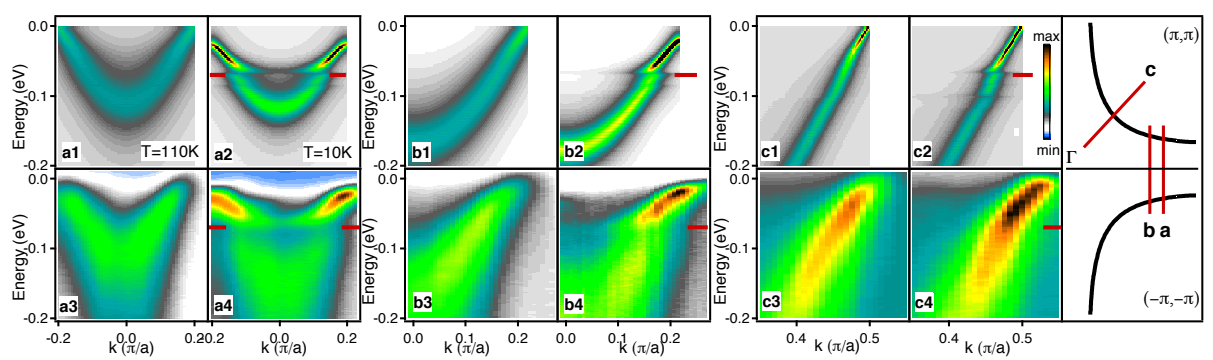

Figure 1.8: Calculated versus measured spectral function $A(\vec{k}, \omega)$ in $\mathrm{Bi}_{2} \mathrm{Sr}_{2} \mathrm{Ca}_{0.92} \mathrm{Y}_{0.08} \mathrm{Cu}_{2} \mathrm{O}_{8+\delta}$ from Devereaux et al. (2004). The upper row shows the results of the calculations and the lower row the results of the measurements. The pictures with the numbers 1 and 2 show a normal (not superconducting) state of the system. The numbers 3 and 4 show a superconducting state of the system and the red lines denote a phonon mode with approximately constant energy. The electrons and the phonon mode interact and form quasiparticles within the shown band structure. The letters of the plots (a, b, and c) are explained in the last column, where the position of the momenta in the Brillouin zone of a, b, and c is shown, respectively. One can see that both the calculation and the measurement show the same features. The difference is that the measured data is considerably broadened compared to the calculated ones. Reprinted figure with permission from Devereaux et al. (2004) (C) 2004 by the American Physical Society.

For this thesis, it is important, because $A(\vec{k}, \epsilon-\hbar \omega)$ grants access to the momentum distribution via

$$
n(\vec{k})=\int d \epsilon G^{<}(\vec{k}, \epsilon)=\int d \epsilon f(\epsilon) A(\vec{k}, \epsilon)
$$

However, only with the angle-resolved version of PES discussed in the next section, one is able to access the momentum dependence of the spectral function, which is necessary to calculate the momentum distribution.

\subsubsection{Angle-resolved photoemission spectroscopy}

In the previous section, we introduced the theoretical basis for a PES measurement. By additionally measuring the angle of the emitted electron, one can reconstruct its initial momentum $\vec{k}$, which is equal to the momentum of the electron in the solid, because the momentum of the photon is negligible. In this way, one obtains access not only to the momentum dependence of the spectral function. However, a realistic, measured intensity $P_{\text {ARPES }}(\vec{k}, \epsilon)$ is broadened in momentum and energy, which is modeled by convolutions with the Gaussian functions $R_{M}(\vec{k})$ and $R_{E}(\epsilon)$, respectively. Additionally, there is an approximately constant background $b$. This leads to

$$
P_{\mathrm{ARPES}}(\vec{k}, \epsilon) \approx \int d \epsilon^{\prime} d^{3} k^{\prime} \tau^{2}(\epsilon) f\left(\epsilon^{\prime}\right) A\left(\vec{k}^{\prime}, \epsilon^{\prime}\right) R_{M}\left(\vec{k}-\vec{k}^{\prime}\right) R_{E}\left(\epsilon-\epsilon^{\prime}\right)+b,
$$

see Damascelli et al. (2003).

We give an example for an ARPES measurement in Fig. 1.8. It shows a calculation of the spectral function $A(\vec{k}, \omega)$ and the measurement. One can see that 

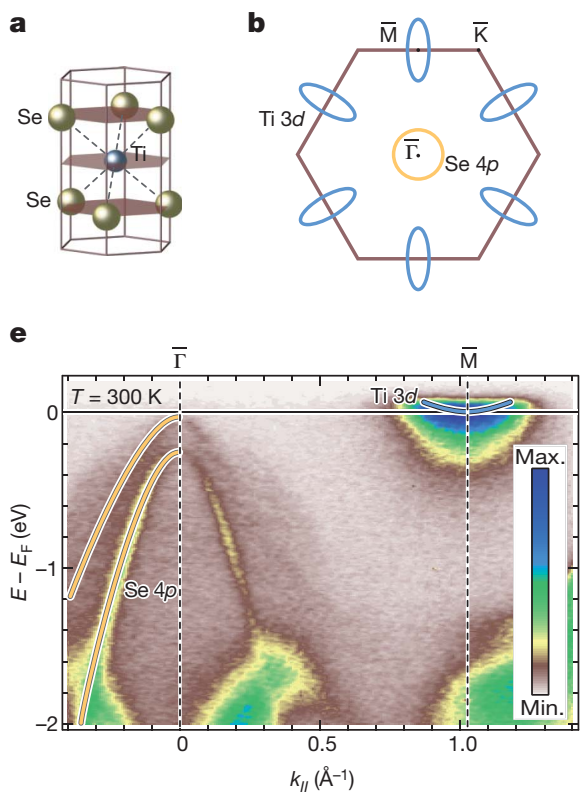

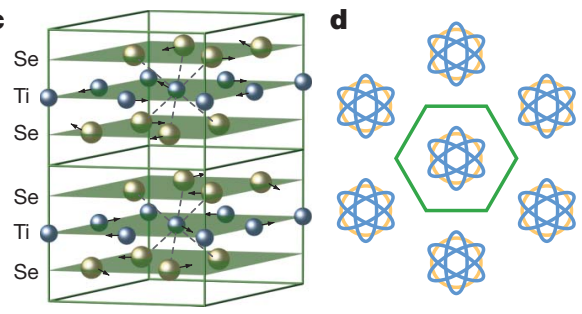

$\mathbf{f}$

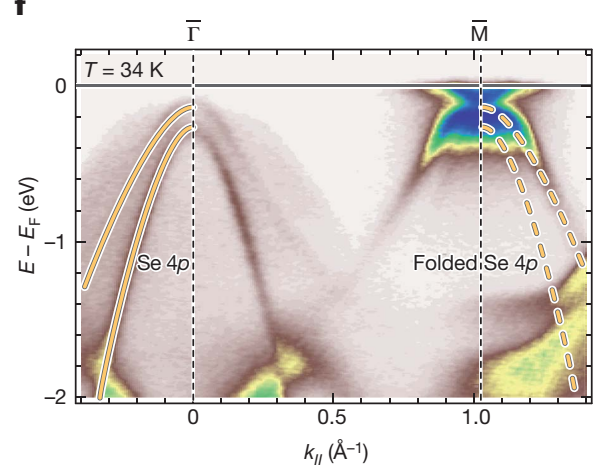

Figure 1.9: CDW phase transition of $1 \mathrm{~T}-\mathrm{TiSe}_{2}$ : The left half show the unit cell (a), Brillouin zone (b) and ARPES measurement (e) for the normal state at room temperature. The right half $(\mathrm{c}, \mathrm{d}, \mathrm{f})$ shows the same for the CDW state at $34 \mathrm{~K}$. One can see that the hole-like SE-4p bands are folded from $\bar{\Gamma}$ to $\bar{M}$. The color scale measures the number of electrons emitted from the sample. Reprinted by permission from Macmillan Publishers Ltd: Nature (Rohwer et al., 2011), (c)2011.

experiment and theory agree and reveal the same electronic structure. However, the measured distribution is broadened compared to the calculated images.

Another drawback, which occurs in angle-resolved measurements, is connected to the wave vector $\vec{k}$ of the electron: the component $\vec{k}_{\|}$parallel to the sample surface can be determined exactly, while the component $\vec{k}_{\perp}$ perpendicular to the surface cannot. This stems from the fact that $\vec{k}_{\perp}$ is not conserved across the surface because of the missing translational invariance (Damascelli et al., 2003). However, for the investigation of (quasi) two- or one-dimensional samples, this is not a problem. Therefore, we will not address this problem here, because ultimately, this thesis is concerned with low-dimensional systems.

\subsubsection{Time- and angle-resolved photoemission spectroscopy}

It is also possible to track the evolution of the electronic occupation. This is known as time-resolved ARPES (trARPES). An abundant number of timeresolved photoemission spectroscopy experiments have been performed, for example by Perfetti et al. (2007); Schmitt et al. (2008); Graf et al. (2011); Rohwer et al. (2011); Rettig et al. (2012); Smallwood et al. (2012); Liu et al. (2013). In trARPES experiments, the sample is first excited and then measured after a delay time. Both can be achieved using laser beams. One can perform such ex- 

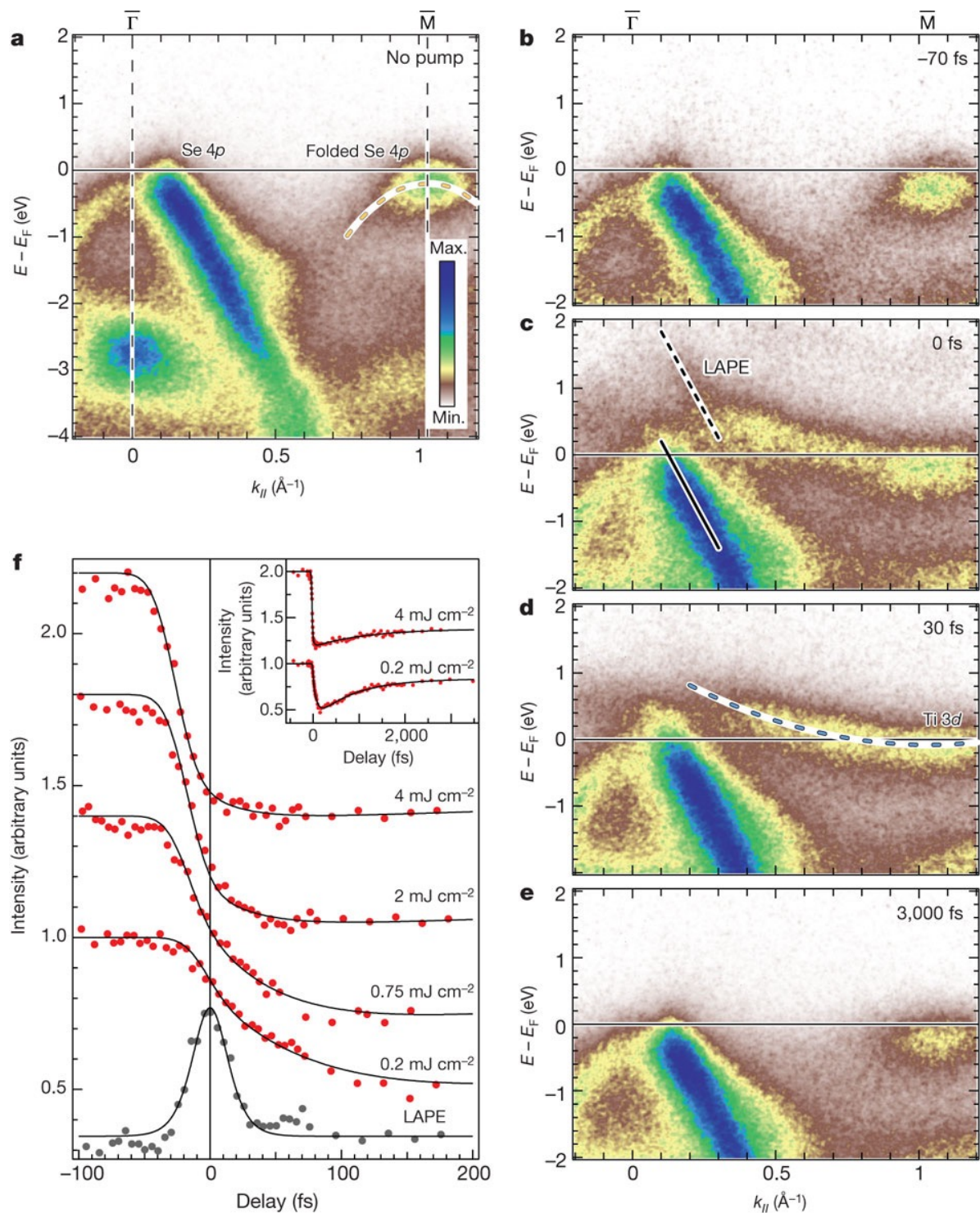

Figure 1.10: Snapshots of the time evolution of an ARPES intensity measurements (Rohwer et al., 2011) of $1 \mathrm{~T}-\mathrm{TiSe}_{2}$. They monitored the CDW phase of the material excited by infrared laser pulses $\left(E_{\text {photon }}^{\text {pump }}=1.57 \mathrm{eV}\right)$. The sample was probed by femtosecond pulses with extreme ultraviolet light $\left(E_{\text {photon }}=43 \mathrm{eV}\right)$.

a) Charge-density wave phase without the excitation at a temperature of $125 \mathrm{~K}$.

b-e) Snapshots of the measured time evolution. The time in the upper-right corner denotes the pump-probe delay.

f) Integrated intensity of the folded Se $4 \mathrm{p}$ band as a measure for the CDW order. It shows the transient excitations. The CDW order breaks down immediately. The inset shows that it recovers on a time scale of the order of picoseconds.

Reprinted by permission from Macmillan Publishers Ltd: Nature (Rohwer et al., 2011), (C) 2011 . 
periments by varying the time between excitation and measurement beam (Liu et al., 2013). The shorter the laser pulses, the higher is the time resolution.

As an example for this technique, we present the experiment performed by Rohwer et al. (2011), who investigate a two-dimensional compound. It is a monolayer of the transition metal dichalcogenide $1 \mathrm{~T}-\mathrm{TiSe}_{2}$, where $1 \mathrm{~T}$ denotes the polytype of the layer (Kolobov and Tominaga, 2016). This material is in a charge-ordered state, which occurs as a charge-density wave (CDW) at temperatures below $200 \mathrm{~K}$. There, the atoms have an equilibrium position, so that the unit cell doubles in all directions compared to higher temperatures. This leads to the Se $4 \mathrm{p}$ band being folded from $\bar{\Gamma}$ (center of the Brillouin-zone) to $\overline{\mathrm{M}}$ (edge of the Brillouin zone), which is shown in Fig. 1.9. Therefore, in this experiment, the occupation of the folded Se $4 \mathrm{p}$ band is used as measure for the order of the CDW.

Fig. 1.10 shows the time evolution of the measured intensity. During the experiment, they probe the sample by extreme ultraviolet light $E_{\text {photon }}=43 \mathrm{eV}$. At a certain time, the sample is excited by a probe beam. They observe that electrons are shifted into the $\mathrm{Ti} 3 \mathrm{~d}$ band. The excitation is instantaneous, which can be seen in Fig. 1.10c, where the delay between the pump and the probe beam is $0 \mathrm{fs}$. Fig. $1.10 \mathrm{f}$ displays the occupation of the folded Se $4 \mathrm{p}$ band. It instantaneously changes, and the CDW order is reduced in an instant. The inset of the figure shows that the order recovers on times of $\mathcal{O}(1 \mathrm{ps})$.

This is an example for the benefits of time-resolved ARPES. The evolution of the non-equilibrium physics of electrons can be studied. Furthermore, one can observe relaxation processes, which we will investigate from the theoretical point of view in this thesis.

\subsection{4 (Quasi) one-dimensional materials}

There are materials which exhibit one-dimensional features. By construction, carbon nanotubes (Egger and Gogolin, 1997; Kane et al., 1997; Egger and Gogolin, 1998) are one-dimensional on a microscopic level. There are also solids, which have one-dimensional electronic patterns embedded in their threedimensional structure. In these materials, there are chains made up of atoms or molecules, where the coupling within the chain is distinctly stronger than the other couplings. For example, in superconducting cuprates like $\mathrm{SrCuO}_{2}$ (Keren et al., 1993; Motoyama et al., 1996; Kojima et al., 1997) and $\mathrm{YBa}_{2} \mathrm{Cu}_{2} \mathrm{O}_{4}$ (Karpinski et al., 1988; Kaldis et al., 1989; Zhou and Goodenough, 1996), chains of $\mathrm{CuO}$ build up $\mathrm{CuO}_{2}$ planes. Further examples for quasi one-dimensional compounds are $\mathrm{K}_{0.3} \mathrm{MoO}_{3}$ (Travaglini et al., 1981), Bechgaard salts, and an organic conductor abbreviated by TTF-TCNQ (Jérome and Schulz, 1982). The latter stands for a complex formed by tetrathiafulvalene (TTF, $\left.\mathrm{C}_{6} \mathrm{H}_{4} \mathrm{~S}_{4}\right)$ and tetracyanoquinodimethane (TCNQ, $\mathrm{C}_{12} \mathrm{H}_{4} \mathrm{~N}_{4}$ ). As a last example, Blumenstein et al. (2011) were able to manipulate gold on a solid surface, thereby creating gold stripes, and measured the system with ARPES. Also for the previous examples, photoemission spectroscopy studies were performed. E.g., Ishii et al. (2003) considered carbon nanotubes, Kim et al. (1996), Kim et al. (2006), and Koitzsch et al. (2006) $\mathrm{SrCuO}_{2}$, Kondo et al. (2010) $\mathrm{YBa}_{2} \mathrm{Cu}_{2} \mathrm{O}_{4}$, Perfetti et al. 
(a)

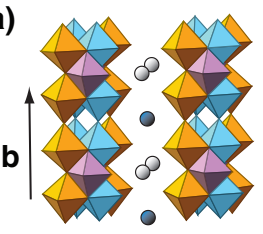

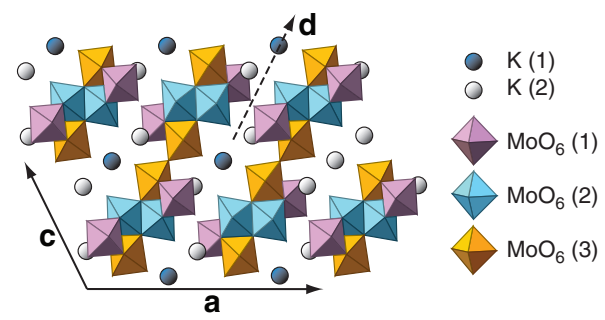

(b)

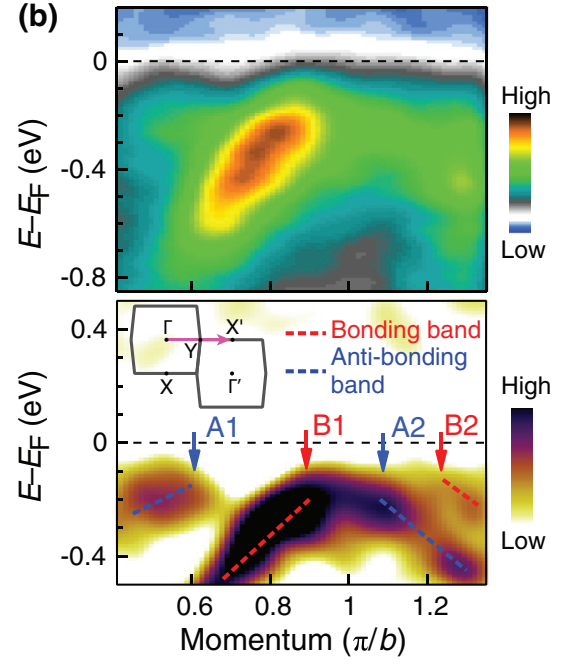

(c)

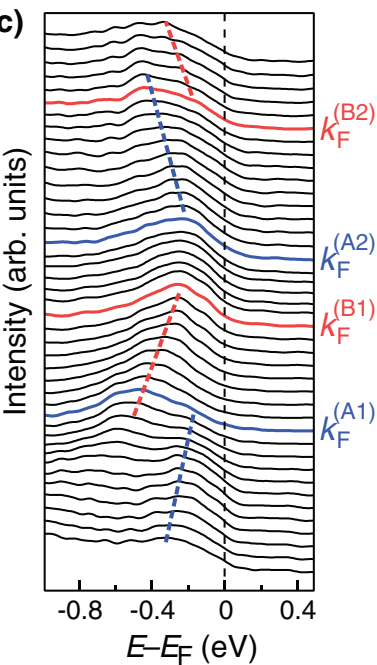

Figure 1.11: Crystal structure and static band properties of $\mathrm{K}_{0.3} \mathrm{MoO}_{3}$. (a) Chains (left) of $\mathrm{MoO}_{6}$ in $b$ directions building up cleavage planes (right). (b) Upper picture: ARPES intensity map $I$ of the unperturbed sample at a temperature of $20 \mathrm{~K}$. Lower picture: Plot of $\partial I^{2} / \partial E^{2}$, which brings out the band structure. The material has four bands near the Fermi-edge (A1, A2, B1, and B2). They are marked in the plot by dashed lines. Their corresponding Fermi momenta are denoted by $k_{\mathrm{F}}^{(\mathrm{A} 1)}$ and $k_{\mathrm{F}}^{(\mathrm{B} 1)}$, $k_{\mathrm{F}}^{(\mathrm{A} 2)}$, and $k_{\mathrm{F}}^{(\mathrm{B} 2)}$ (c) Individual energy distribution curves used to create (b). Again the four bands are marked. Reprinted figure with permission from Liu et al. (2013) (c) 2013 by the American Physical Society.

(2002) and Liu et al. (2013) $\mathrm{K}_{0.3} \mathrm{MoO}_{3}$, Zwick et al. (1997) Bechgaard salts, and Claessen et al. (2002) and Sing et al. (2003) TTF-TCNQ.

Such effectively one-dimensional materials exhibit a lot of fascinating effects. For example, in carbon nanotubes features of a Luttinger-liquid have been observed (Ishii et al., 2003). The one-dimensional structures of $\mathrm{SrCuO}_{2}$ are approximate Heisenberg chains (Keren et al., 1993; Motoyama et al., 1996; Kojima et al., 1997). Below $180 \mathrm{~K}, \mathrm{~K}_{0.3} \mathrm{MoO}_{3}$ shows the Peierls transition to a charge-density wave and a gap opening at the Fermi-level (Travaglini et al., 1981; Johnston, 1984). The Bechgaard salt (TMTSF $)_{2} \mathrm{PF}_{6}$ was the first organic superconductor (Jérome, D. et al., 1980). These materials exhibit a spin-charge wave below $12 \mathrm{~K}$ (Dressel, 2003). The spectra of the TTF-TCNQ compound are perfectly described by a one-dimensional Hubbard model at metallic doping and exhibits a charge-density wave below $54 \mathrm{~K}$ (Claessen et al., 2002; Sing et al., 2003; Benthien et al., 2004; Seabra et al., 2014). 

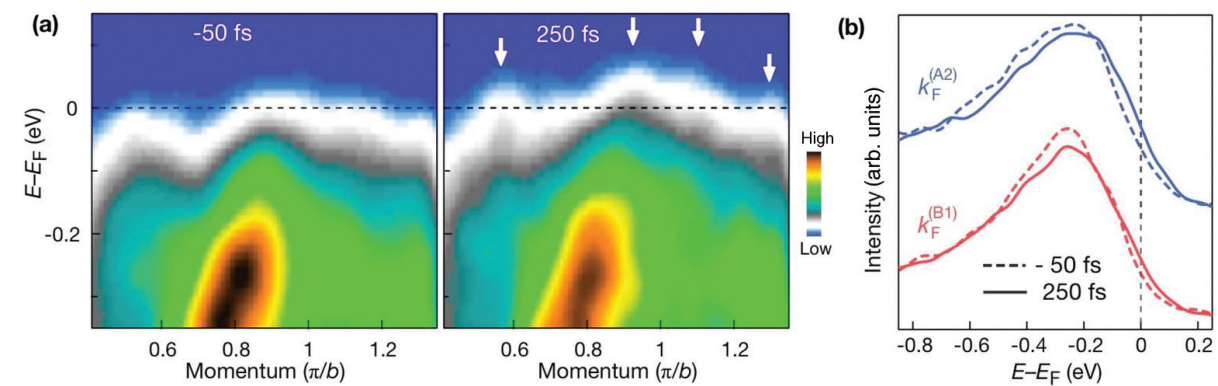

Figure 1.12: ARPES intensity for $\mathrm{K}_{0.3} \mathrm{MoO}_{3}$. (a) Intensity maps for delay times of $-50 \mathrm{fs}$ and $250 \mathrm{fs}$, (b) Intensity at the Fermi momenta $k_{\mathrm{F}}^{(\mathrm{B} 1)}$ and $k_{\mathrm{F}}^{(\mathrm{A} 2)}$. Reprinted figure with permission from Liu et al. (2013) (C)2013 by the American Physical Society.

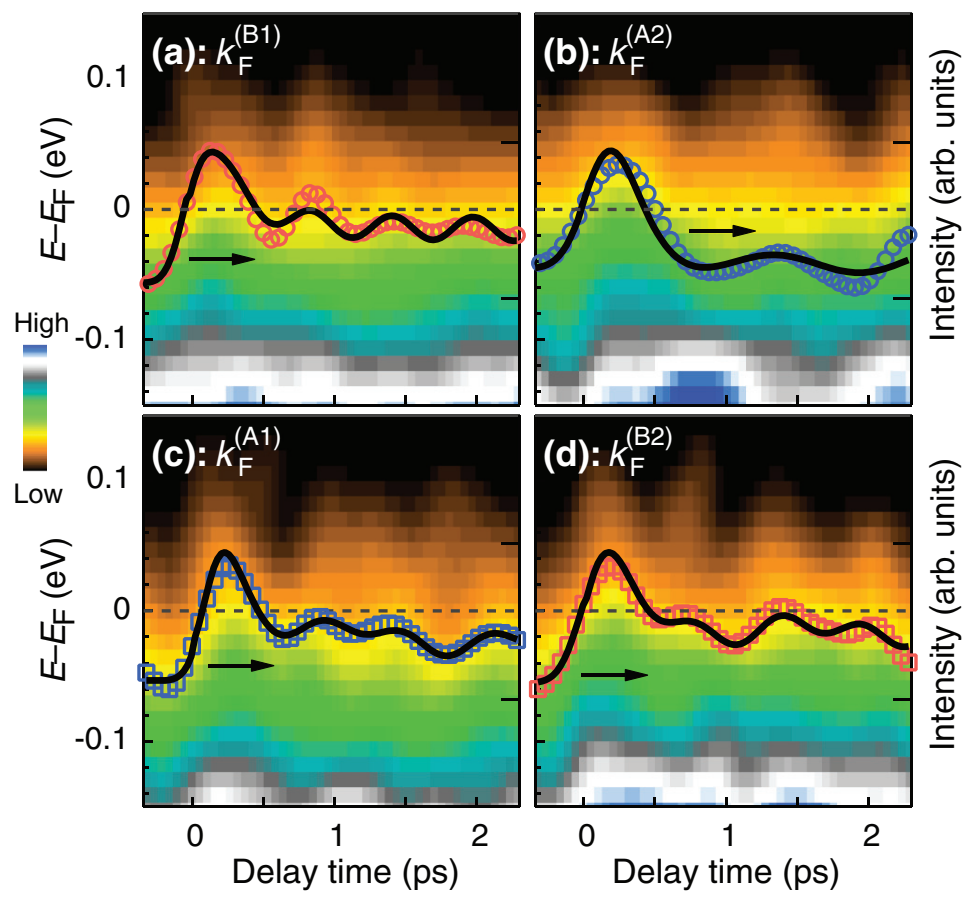

Figure 1.13: Time evolution of the electronic structure of $\mathrm{K}_{0.3} \mathrm{MoO}_{3}$ near the Fermi edge for each of the Fermi momenta $k_{\mathrm{F}}^{(\mathrm{A} 1)}, k_{\mathrm{F}}^{(\mathrm{B} 1)}, k_{\mathrm{F}}^{(\mathrm{A} 2)}$, and $k_{\mathrm{F}}^{(\mathrm{B} 2)}$. The symbols are the intensity integrated over the energy from -50 to $100 \mathrm{meV}$. One can see the onset of relaxation, while the occupation is oscillating. The lines are fits with functions that are a combination of an exponential and a sine function, with which the frequencies of the oscillations were extracted: (a) $1.7 \mathrm{THz}$, (b) $0.8 \mathrm{THz}$, (c, d) both. Reprinted figure with permission from Liu et al. (2013) (c)2013 by the American Physical Society. 
As an example for trARPES performed for a quasi one-dimensional system, we briefly discuss the experiment of Liu et al. (2013), who investigated $\mathrm{K}_{0.3} \mathrm{MoO}_{3}$. The structure of the quasi one-dimensional material is shown in Fig. 1.11a. Near the Fermi-edge, it exhibits the four bands A1, A2, B1, and B2, which is confirmed by the various plots of Figs. $1.11 \mathrm{~b}$ and c. In this experiment, intensity maps were recorded like those in Fig. 1.12.

Fig. 1.13 shows their result, i.e. the dynamics of the energy distribution for the four Fermi momenta $k_{\mathrm{F}}^{(\mathrm{A} 1)}, k_{\mathrm{F}}^{(\mathrm{B} 1)}, k_{\mathrm{F}}^{(\mathrm{A} 2)}$, and $k_{\mathrm{F}}^{(\mathrm{B} 2)}$. The latter are labeled by their respective band. One can clearly see oscillations during the onset of an overall relaxation process. This is quantified by the symbol plots, because they show the intensity at the Fermi-momentum integrated over the energy from $-50 \mathrm{meV}$ to $100 \mathrm{meV}$. The fits (solid lines) reveal two different oscillation frequencies $(0.8 \mathrm{THz}$ and $1.7 \mathrm{THz})$. Furthermore, one can see that the electrons are not relaxed during the relatively long measurement time of about 2 ps. Although the ARPES measurements are broadened, they show that it is possible to track the thermalization. This experiment shows that one is able to track the time evolution of one-dimensional, real materials.

\section{Summary}

In this chapter, we have shown how the momentum distribution in combination with the band structure can be measured. The measurements are considerably broadened, but they reveal the same features as numerical calculations. Furthermore, we have presented an example for the observation of time evolution of the electronic occupation. Moreover, we have pointed out the existence of one-dimensional materials, which can be investigated by photoemission spectroscopy. Hence, the time evolution of momentum distributions in (quasi) onedimensional materials are accessible and a useful measure for the investigation of the electronic relaxation in solids. 


\subsection{Thermalization in closed quantum systems: Theoretical overview}

In this thesis, we are concerned with the long time dynamics of closed manybody systems. In the previous section, we presented the experimental motivation for this work. It shows that the long time dynamics may not lead to thermalization within the time of an experiment. For a further investigation of this, we now introduce the theoretical basis for calculating the long time evolution of large quantum many-body systems.

As a first step, we consider a well known treatment of large quantum systems, i.e. thermodynamics. There, thermal equilibrium is assumed, which means that the system is in a state described by a certain mixed state. In a closed system, the density matrix of this mixed state is (Balian, 2007)

$$
\rho_{\mathrm{mc}}=\frac{1}{Z_{\mathrm{mc}}} \sum_{E-d E \leqslant E_{\alpha} \leqslant E+d E}|\alpha\rangle\langle\alpha|,
$$

where $|\alpha\rangle$ are the energy eigenstates, i.e. the eigenstates of the Hamiltonian $\hat{H}$. Evidently, $\rho_{\mathrm{mc}}$ is an indifferently weighted composition of all energy eigenstates in a small energy shell, so that there is a defined energy $E$ in the system. This is known as the microcanonical ensemble. Moreover, the normalization factor $Z_{\mathrm{mc}}=\operatorname{tr}\left(\sum_{E-d E \leqslant E_{\alpha} \leqslant E+d E}|\alpha\rangle\langle\alpha|\right)$ is the microcanonical partition function.

Now we bring the investigated system (S) in contact with a heat bath (B) By definition, B has to be much larger than $\mathrm{S}$. The equilibrium state of $\mathrm{S}$ is obtained by maximizing the entropy (Jaynes, 1957a,b; Balian, 2007)

$$
S[\rho]=-k_{\mathrm{B}} \operatorname{tr}(\rho \ln \rho) .
$$

Hereby, the energy of $\mathrm{S}$ is not fixed but only its expectation value, which leads to the constraint $\langle\hat{H}\rangle=\operatorname{tr}(\rho \hat{H})$. The method of performing this maximization with constraints is the Lagrangian multipliers scheme. The Lagrangian multiplier for the energy constraint is the inverse temperature $\beta$. The result is (Balian, 2007)

$$
\rho_{\text {can }}=\frac{1}{Z_{\text {can }}} e^{-\beta \hat{H}} .
$$

This expression matches the density matrix obtained by taking the partial trace over the bath, $\operatorname{tr}_{\text {bath }} \rho_{\text {full }}$, if the interaction between system and bath is small. More precisely, the energy stored in the interaction has to be much smaller than both the energies stored in the bath and the one stored in the system (Balian, 2007).

Moreover, one can also add a particle bath, resulting in the grandcanonical ensemble (Balian, 2007). The corresponding equilibrium state is

$$
\rho_{\mathrm{gc}}=\operatorname{tr}_{\mathrm{bath}} \rho_{\mathrm{full}}=\frac{1}{Z_{\mathrm{gc}}} e^{-\beta(\hat{H}-\mu \hat{N})}
$$

Here, neither energy nor the total particle number are fixed, so that the constraints for maximizing the entropy are $\langle\hat{H}\rangle=\operatorname{tr}(\rho \hat{H})$ and $\langle\hat{N}\rangle=\operatorname{tr}(\rho \hat{N})$. The 
respective Lagrangian multipliers are $\beta$ and $\beta \mu$, where $\mu$ is the chemical potential. It measures how much energy is needed to add a particle to the system.

However, a system may not be in a state described by thermal density operators like Eqs. (1.58), (1.60), and (1.61). There are examples, in which systems do not exhibit signs of thermalization over a long time, for instance in the collapse and revival or the Newton's cradle experiments (Greiner et al., 2002b; Kinoshita et al., 2006) introduced in Sec. 1.1.6.

Hence, it is important to learn more about non-equilibrium quantum mechanics. A major issue in this field is that pure states always stay pure in the quantummechanical unitary time evolution $|\psi(t)\rangle=e^{-i \hat{H} t / \hbar}|\psi(t=0)\rangle$, see Eq. (1.2). Thus, the question arises how thermalization is established, knowing that a pure state can never approach a mixed state like a thermal one.

An important point is that a quantum state cannot be observed in experiments. The only measurable objects are the eigenvalues of observables (Basdevant and Dalibard, 2002).

Another point is realizing that there is no well-defined, long-time limit of a pure state $|\psi\rangle=\sum_{\alpha} C_{\alpha} e^{-i E_{\alpha} t / \hbar}|\alpha\rangle$ because of the oscillating factors $e^{-i E_{\alpha} t / \hbar}$. Therefore, also expectation values of observables have no well-defined long-time limit. Thus, one needs to refrain from the question of what happens at infinite time, but one should rather investigate the following question: How does the system behave most of the time? For this reason, time averages are investigated. In this way, the diagonal ensemble (Deutsch, 1991; Rigol et al., 2008) is found. The long time average of an expectation value of an observable $\hat{O}$ in a pure state $|\psi\rangle=\sum_{\alpha} C_{\alpha} e^{-i E_{\alpha} t / \hbar}|\alpha\rangle$ is

$$
\begin{aligned}
\lim _{T \rightarrow \infty} \frac{1}{T} \int_{0}^{T} d t\langle\psi|\hat{O}| \psi\rangle & =\sum_{\alpha, \beta}\langle\alpha|\hat{O}| \beta\rangle C_{\alpha}^{*} C_{\beta} \lim _{T \rightarrow \infty} \frac{1}{T} \int_{0}^{T} d t e^{-i\left(E_{\beta}-E_{\alpha}\right) t / \hbar} \\
& =\sum_{\alpha}\langle\alpha|\hat{O}| \alpha\rangle\left|C_{\alpha}\right|^{2}=: \operatorname{tr}\left(\rho_{\operatorname{diag}} \hat{O}\right)
\end{aligned}
$$

Obviously, the off-diagonal elements $\langle\alpha|\hat{O}| \beta\rangle, \alpha \neq \beta$ do not play a role because of dephasing, i.e. $\lim _{T \rightarrow \infty} \frac{1}{T} \int_{0}^{T} d t e^{-i\left(E_{\beta}-E_{\alpha}\right) t / \hbar}=0$. This is only true if the Hamiltonian exhibits level repulsion ${ }^{2}$, which is the case if the systems are not integrable $^{3}$ (Santos and Rigol, 2010; Polkovnikov et al., 2011). Eq. (1.62) defines the diagonal ensemble (Deutsch, 1991; Rigol et al., 2008)

$$
\rho_{\text {diag }}=\sum_{\alpha}\left|C_{\alpha}\right|^{2}|\alpha\rangle\langle\alpha|
$$

It is a first step towards an ensemble description, despite the fact that the initial state is pure. However, due to its strong dependence on the initial state, it is not an equilibrium description in general.

The dependence of the diagonal ensemble on the initial state is weakened if the eigenstate thermalization hypothesis (ETH) (Deutsch, 1991; Srednicki, 1994;

\footnotetext{
${ }^{2}$ Level repulsion means that there is a non-zero minimum energy difference between any pair of energy eigenstates, i.e. $\left|E_{\alpha}-E_{\beta}\right|>0 \forall(\alpha, \beta)$.

${ }^{3}$ Integrable systems have a large number of conserved quantities. We introduce the notion of integrability later on.
} 
Rigol et al., 2008; Rigol, 2009) holds and the initial state is composed of energy eigenstates within an energy shell, $|\psi\rangle=\sum_{E-d E \leqslant E_{\alpha} \leqslant E+d E} C_{\alpha} e^{-i E_{\alpha} t / \hbar}|\alpha\rangle$. The ETH states that the expectation value of a few-body observable $\hat{O}$ in an energy eigenstate is a smooth function of the energy of the eigenstate (Rigol, 2009; Rigol and Srednicki, 2012):

$$
\langle\alpha|\hat{O}| \alpha\rangle=O\left(E_{\alpha}\right) .
$$

A few-body operator is one that contains products of a small number of creation and annihilation operators. An example for a few-body operator is the number operator, be it in real or momentum space, or the total number of particles. In contrast to this, a many-body operator is, for instance, the exponential of a few-body operator, such as the time-evolution operator. The reason is its series expansion, because it contains operators with $2 n$ creation and annihilation operators with all possible $n \in \mathbb{N}_{0}$.

Employing Eq. (1.64), the long time average in Eq. (1.62) becomes

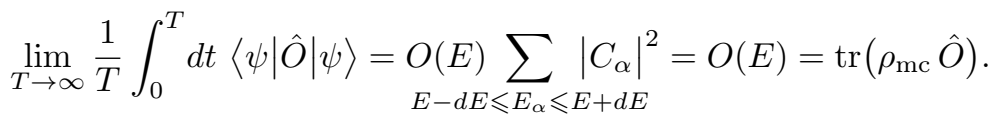

Of course, this statement is restricted to systems where the ETH holds, to few-body operators, and to initial states, which are a composition of energy eigenstates picked from a small energy shell. However, the idea of the ETH tempts one to define a system as thermalized when the expectation values of fewbody observables are (approximately) equal to the thermal expectation values for most of the times.

A more general statement about non-equilibrium quantum mechanics of pure states is that, in large systems, nearly all pure states are locally thermal (Popescu et al., 2006). Thus, after tracing out a much larger part of the system, the resulting density matrix is very close to being thermal. Of course, pure states stay pure for all times, but during the time evolution of a generic pure state, it is very probable that this state will be locally thermal for most times. This was later formulated as the local relaxation conjecture (Cramer et al., 2008a). Therefore, a reasonable definition of thermalization is that the state is thermal in all small, finite subsystems.

In summary, triggered by the vast amount of publications and opinions regarding thermalization presented in this chapter, for the author of this thesis, there is no fixed definition of thermalization. There are strong and weak definitions of thermalization. They heavily depend upon the requirements of an experiment. The narrowest definition of thermalization one can make is that the initial quantum mechanical state has to evolve into a thermal ensemble. This is of course neither the case for pure states nor for mixed states, except for the thermal states. Thus, this definition is not very useful for closed quantum systems. Consequently, one has to find weaker definitions of thermalization. We have shown for instance that local thermalization of the state can be used as a definition. However, there is also the possibility to abandon the consideration of states in that matter and to restrict oneself to objects that can be measured in experiments instead. Hence, we understand a state to be thermalized when the expectation values of observables have thermalized, i.e. $\operatorname{tr}[\rho(t) \hat{O}]=\operatorname{tr}\left[\rho_{\text {thermal }} \hat{O}\right]$. 
This is also a very narrow definition, because, surely, one can construct an observable in such a way that it does not thermalize. Therefore, this definition should also be weakened. Like mentioned before, a reasonable definition is that a state is considered to be thermal when all few-body observables have thermalized.

For this work the relevant observables to measure thermalization are the number operators in momentum space $\hat{n}_{k}=\hat{c}_{k}^{\dagger} \hat{c}_{k}$. Their expectation values give the momentum distribution. The use of the momentum distribution as an indicator for thermalization is of course not a new notion, as it has been extensively used before (Srednicki, 1994; Manmana et al., 2007; Moeckel and Kehrein, 2008; Eckstein et al., 2009; Rigol et al., 2007; Rigol, 2009; Kinoshita et al., 2006). In this thesis, we will introduce a Boltzmann equation that describes the time evolution of the momentum distribution of the quasiparticles of the system, and we will investigate its tendency to thermalize.

As we mentioned before, there is a prominent class of systems that do not show signs of thermalization, which are known as integrable systems. These kind of systems include a number of conserved quantities, which grows with system size. In Sec. 1.1.6, we already discussed two examples for such systems: The collapse-and-revival experiment of Greiner et al. (2002b) and the quantum Newton's cradle performed by Kinoshita et al. (2006).

The equilibrium density matrix of an integrable theory is found by the same principle as Eq. (1.60) and Eq. (1.61), namely by maximizing entropy with respect to the constraints $\left\langle\hat{Q}_{j}\right\rangle=\operatorname{tr}\left(\rho \hat{Q}_{j}\right)$, where $\hat{Q}_{j}$ are the conserved quantities. The maximization with constraints is performed by the Lagrangian multipliers method. It leads to the ensemble, which is known as generalized Gibbs ensemble (GGE) (Rigol et al., 2007, 2008)

$$
\rho_{\mathrm{GGE}}=\frac{1}{Z_{\mathrm{GGE}}} \exp \left\{-\sum_{j} \beta_{j} \hat{Q}_{j}\right\},
$$

where $\beta_{j}$ are the Lagrangian multipliers corresponding to $\hat{Q}_{j}$.

The integrability of a model can be broken by changing its Hamiltonian. Then, the quantities $\hat{Q}_{j}$ are no longer conserved. If integrability is broken slightly, one typically sees a special effect called prethermalization (Eckstein et al., 2009; Fagotti, 2014; Bertini and Fagotti, 2015; Gring et al., 2012). The expectation value of the investigated few-body operator relaxes after a relatively small time, but the value is not thermal and seems to be stationary for a relatively long time. The reason for this effect is that the quantities $\hat{Q}_{j}$ are still approximately conserved, relax very slowly, and thus prevent thermalization for a very long time. During the prethermalization, the expectation values match the prediction of the GGE, which assumes the quasi-conserved quantities to be conserved. However, on a longer time scale, thermalization is expected (Berges et al., 2004; Eckstein et al., 2009; Bertini and Fagotti, 2015; Gring et al., 2012).

The experiment of Kinoshita et al. (2006) is close to being integrable apart from minor issues. The interaction is not exactly a point-interaction as in the corresponding integrable model and also the previously mentioned heating, particle loss, and anharmonicity effects give rise to non-integrability. Thus, this experiment is an example for systems with weakly broken integrability. Nevertheless, 
despite the broken integrability, the features of the corresponding integrable system are clearly visible. This suggests that the effects of integrability are not immediately gone when integrability is broken, but they slowly wear off the further away the theory departs from an integrable point. To have a better understanding of how a small integrability breaking influences the long time dynamics, we investigate a model with weakly broken integrability.

We will study how the thermalization time depends on the integrability-breaking parameter. In order to do this, we need a method that can reach very long times. They must be so long that one can reach thermalization even for very small integrability breaking. There are many methods for calculating the time evolution of a one-dimensional quantum mechanical system. However, most of them can only investigate small systems or lack the ability to reach times long enough to observe thermalization.

An important analytical approach is the flow equation or continuous unitary transformation (CUT) method (Wegner, 1994; Głazek and Wilson, 1993, 1994; Kehrein, 2006), where the Hamiltonian is gradually diagonalized by a sequence of small, unitary transformations. By this procedure, the time evolution becomes very simple, but simple observables are transformed into very complicated objects. Thus, in practice one considers a small interaction strength to describe these objects perturbatively (Moeckel and Kehrein, 2008; Essler et al., 2014). Hence, there is also a maximum time scale on which the results are valid.

Another more naive approach to gain information about the time evolution is an expansion in time. For example, Hamerla and Uhrig (2013) studied the expansion of the Heisenberg equations of motions for the creation operator in a one-dimensional Hubbard model. Depending on how many terms one takes into account, the maximum time, for which the computation is valid, increases. However, this approach is not able reach very long times.

Besides analytical methods, there are also numerical methods. The most obvious one is exact diagonalization (Rigol, 2009; Roux, 2009; Santos and Rigol, 2010; Essler et al., 2014). It is an exact approach, but, as we mentioned before, it can only be used for computing the time evolution of rather small systems, even when using super-computers.

A very successful exact numerical approach is the time-dependent density matrix renormalization group method (tDMRG) (White, 1992, 1993; Daley et al., 2004; Manmana et al., 2005; Schollwöck, 2005, 2011). Originally designed for finding ground states of one-dimensional systems, it can calculate the time evolution of a state. For example, tDMRG and CUT have been compared calculating a nearest-neighbor Green's function. The results cannot be distinguished by the bare eye (Essler et al., 2014). With this method, one can compute much larger systems compared to exact diagonalization. However, the maximal time it can reach is limited by growing entanglement and at some point finite size effects set in. Therefore, the long time scale of thermalization cannot be reached (Manmana et al., 2007; Daley et al., 2004; Kollath et al., 2007; Cramer et al., 2008b; Essler et al., 2014).

In the next section, we will introduce our approach for studying a many-body quantum system for long times. Moreover, we will elaborate on the goals of this thesis. 


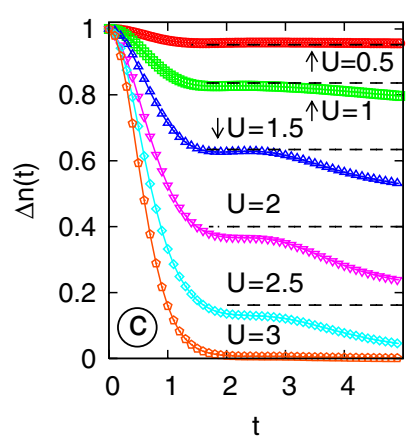

Figure 1.14: Time evolution of the quasiparticle residue in a Hubbard model for several interaction strenghs $U \leqslant 3$. The colored symbols connected by lines are the result of the numerical calculation of Eckstein et al. (2009). One can clearly see the prethermalization plateau. This confirms the findings of Moeckel and Kehrein (2008). Their prediction of the prethermalization plateau are the dashed lines. Reprinted figure with permission from Eckstein et al. (2009) (C)2009 by the American Physical Society.

\subsection{Goals and scope}

In this thesis, we investigate the long time evolution of a many-body quantum system. The previous section showed the difficulties of such an undertaking. In order to tackle this problem, we need to find a measure for thermalization, with which we are able to calculate for long times. Hence, we consider the quasiparticle momentum distribution $n(k, t)$. Our method of choice to study $n(k, t)$ is a quantum Boltzmann equation.

The motivation for such an approach is the physical picture that is depicted in the work of Moeckel and Kehrein (2008). For small times, quasiparticles are building up. On a larger time scale, the quasiparticles interact until they finally thermalize. This interaction and equilibration of the quasiparticle momentum distribution is described by a Boltzmann equation (Moeckel and Kehrein, 2008). In their work, they investigated a Hubbard model ${ }^{4}$ in $d \geqslant 2$ dimensions, which is integrable if the interaction strength is zero. Their findings were confirmed by a non-equilibrium dynamical-mean-field-theory calculation of Eckstein et al. (2009), see Fig. 1.14. They found prethermalization plateaus for several interaction strengths, which matched the prediction of Moeckel and Kehrein (2008). In general, a Boltzmann equation can be derived for systems, in which the lifetime of the quasiparticles is so long that it can be assumed to be infinite (Rammer and Smith, 1986; Erdös et al., 2004; Moeckel and Kehrein, 2008; Kamenev and Levchenko, 2009).

When certain prerequisites are fulfilled, the collision term of a Boltzmann equation is the leading order contribution of $\dot{n}(k, t)$. We will show in detail in Chapter 2 that $n(k, t)$ evolves according to

$$
\dot{n}(k, t)=\mathcal{I}_{\text {coll }}[n](k, t)+o\left(U^{2}\right),
$$

\footnotetext{
${ }^{4}$ We introduce the Hubbard model in Sec. 1.4.1. There we will also elaborate on its onedimensional version
} 
where $\mathcal{I}_{\text {coll }}$ is the collision term of the Boltzmann equation and $U$ is the interaction strength. The approximations for obtaining a Boltzmann equation will be discussed in Chapter 2. Furthermore, a detailed derivation of the collision term is given in App. B.1.

However, in some cases, the two-particle collision term $\mathcal{I}_{\text {coll }}$ does not lead to thermalization. Yet, this does not exclude the higher-order terms from being able to induce thermalization. Since the higher-order terms are normally much smaller than the leading order, the thermalization of the momentum distribution will accordingly take very long, meaning orders of magnitude longer than naively expected.

An important goal is predicting thermalization times of the quasiparticle momentum distribution for experiments and theoretical studies like for example tDMRG calculations. As pointed out before, thermalization is hardly seen in numerical calculations of one-dimensional systems and we want to contribute to answering why that is the case. With the method we use, one is able to tell if two-particle collisions are ineffective for certain models, which may lead to unexpectedly long thermalization times.

The two models we will investigate are the one-dimensional Hubbard model and a certain magnetic phase of a one-dimensional Manganite chain. Both models will shortly be introduced in the following two subsections. We will elaborate on the two of them in Chapters 3 and 4, respectively.

\subsubsection{The Hubbard model}

The Hubbard model introduced by Hubbard (1963, 1964a,b), Gutzwiller (1963), and Kanamori (1963) describes the electrons of a crystal structure, where the unit cell is made up of one atom. Each atom contributes only two energetically degenerate valence states, which differ in their spin direction. One state has spin $\uparrow$, the other spin $\downarrow$. The Hamiltonian of the Hubbard model in $d$ dimensions (see Eq. (1.34)) reads

$$
\hat{H}_{\text {Hubbard }}=-\underset{\substack{\mathrm{NN}\left(l, l^{\prime}\right) \\ \sigma \in\{\uparrow, \downarrow\}}}{J} \hat{c}_{\sigma, l}^{\dagger} \hat{c}_{\sigma, l^{\prime}}+\text { H.c. }+U \sum_{l \in \mathbb{Z}^{d}} \hat{n}_{\uparrow, l} \hat{n}_{\downarrow, l},
$$

with the creation and annihilation operators $\hat{c}_{\sigma, l}^{\dagger}$ and $\hat{c}_{\sigma, l}$, respectively, and the number operator $\hat{n}_{\sigma, l}=\hat{c}_{\sigma, l}^{\dagger} \hat{c}_{\sigma, l}$. The first sum runs only over pairs of indices of neighboring atoms.

The first term reflects the ability of electrons to hop from atom to atom. It connects the valence electron states of the atoms and forms the band of states, which we mentioned before. The second term models the screened Coulomb interaction of the electrons. Due to charge screening, the effective electronelectron interaction within a sample becomes short-ranged. Hence, we may approximate this interaction so that the electrons only feel a Coulomb repulsion if they reside on the same atom. The Hubbard Hamiltonian in one dimension is

$$
\hat{H}_{\text {Hubbard }}=-J \sum_{\substack{l \in \mathbb{Z}, \sigma \in\{\uparrow, \downarrow\}}} \hat{c}_{\sigma, l}^{\dagger} \hat{c}_{\sigma, l+1}+\text { H.c. }+U \sum_{l \in \mathbb{Z}} \hat{n}_{\uparrow, l} \hat{n}_{\downarrow, l} .
$$


The one-dimensional Hubbard model is integrable for every $J$ and $U$ (Essler et al., 2005). To investigate the breaking of integrability and its consequence for the equilibration dynamics, we introduce an additional next-to-nearest-neighbor hopping term in the Hamiltonian:

$$
\hat{H}=\hat{H}_{\text {Hubbard }}-J^{\prime} \sum_{\substack{l \in \mathbb{Z}, \sigma \in\{\uparrow, \downarrow\}}} \hat{c}_{\sigma, l}^{\dagger} \hat{c}_{\sigma, l+2}+H . c . .
$$

It accounts for the non-zero overlap of the valence states of next-to-nearestneighbor atoms, and thus enables the electrons to move from one atom to the one after the next. Reasonably, the overlap of next-to-nearest-neighbor atom states is considerably smaller than their nearest-neighbor counterparts.

We will investigate the model in Eq. (1.70) with respect to its long time evolution in the thermodynamic limit by means of a quantum Boltzmann equation. This was previously studied by Fürst et al. (2012, 2013b,a). They examined the time evolution of several initial momentum distributions for the integrable as well as for the non-integrable case. In the integrable case they found non-thermal stationary distributions, to which the momentum distributions converge. This is owed to the integrability of the model. In the non-integrable case, they observed thermalization. In both cases, the relaxation times were measured. Since there is no thermalization in the integrable model, the relaxation time of the integrable case is not the thermalization time (which is infinite for an integrable theory). It can rather be considered a prethermalization time. As argued before, the reason is that models near an integrable point exhibit the dynamics of the integrable point for sufficiently small times. Hence, the relaxation time of the non-integrable case is the prethermalization time of the case with weakly broken integrability. Consistently, Fürst et al. found that this prethermalization time is significantly smaller than the thermalization time of the non-integrable model. We will add a systematic investigation of the thermalization rates to their previous work. Our approach is linearizing the Boltzmann equation around the thermal distribution. The eigenvalues of the resulting linear operator are the relaxation rates, which we are going to find in this thesis.

The Hubbard model has one single-particle band, which can be seen from the kinetic part of the Hamiltonian:

$$
J \sum_{\substack{l \in \mathbb{Z}, \sigma \in\{\uparrow, \downarrow\}}} \hat{c}_{\sigma, l}^{\dagger} \hat{c}_{\sigma, l+1}+J^{\prime} \sum_{\substack{l \in \mathbb{Z}, \sigma \in\{\uparrow, \downarrow\}}} \hat{c}_{\sigma, l}^{\dagger} \hat{c}_{\sigma, l+2}+H . c .=2 \int d k \sum_{\sigma \in\{\uparrow, \downarrow\}} \omega(k) \hat{n}_{\sigma}(k),
$$

with the momentum creation operator $\hat{c}_{\sigma}^{\dagger}(k)=\sum_{l} e^{i 2 \pi k l} \hat{c}_{\sigma l}^{\dagger}$, the momentum number operator $n_{\sigma}(k)=\hat{c}_{\sigma}^{\dagger}(k) \hat{c}_{\sigma}(k)$, and the single-particle band $\omega(k)=$ $J \cos (2 \pi k)+J^{\prime} \cos (4 \pi k)$. We will investigate the case for more than one band, too. In the next section, an effective model with four bands will be introduced, which is suitable for this purpose.

\subsubsection{Praseodymium calcium manganite chain: A multi- band model}

In the last section, the Hubbard model was considered. As we have seen, it consists of exactly one single-particle band. We will also apply our method of 
finding the thermalization rates on a model with more than one band. In order to have more bands, a larger unit cell is needed, because the number of bands is equal to the number of sites in the unit cell.

Our multi-band model is motivated by the material $\mathrm{Pr}_{\mathrm{x}} \mathrm{Ca}_{1-\mathrm{x}} \mathrm{MnO}_{3}$. It is a fascinating material, which we will introduce in detail in Chapter 4. Thereby, we will also point out the property of manganites showing many different magnetic phases. We will consider a one-dimensional effective model of the material and investigate one certain magnetic phase. The effective model describing this phase is similar to the Hubbard model. The difference is that the $\uparrow$ and $\downarrow$ electrons have different energies. These energies alternate in such a way that it leads to a unit cell with four sites.

For the examination of this model, we also use a Boltzmann equation. However, here we need a Boltzmann equation that takes care of more than one bands. Therefore, in Chapter 2, we derive the multi-band Boltzmann equation building on the derivation of the single-band version of Erdös et al. (2004).

\section{Outline}

In this thesis, we consider two one-dimensional models. The first one is the integrable integrable Hubbard model with an additional integrability breaking term in the Hamiltonian, which is a next-to-nearest-neighbor hopping term. This has been previously investigated by Fürst et al. (2012, 2013b). They considered several initial quasiparticle momentum distributions and their relaxation times for different strengths of the next-to-nearest-neighbor hopping amplitude $J^{\prime}$. We want to find the dependence of the thermalization rates on $J^{\prime}$, on the filling and on temperature for any initial quasiparticle momentum distribution in the regime of small integrability breaking. However, we will restrict to the situation, where $\uparrow$ - and $\downarrow$-spin fermions have the same quasiparticle momentum distribution, $n(k)=n_{\sigma}(k)$. This means that the material has no magnetization and the excitation of the system is independent of spin. An example for this is a light-induced excitation, where the number of photons is so huge that there are enough electrons excited to safely assume the momentum distribution's independence of spin. Furthermore, the off-diagonal terms of the matrix-valued Boltzmann equation (Fürst et al., 2012, 2013b) are assumed to have already relaxed to zero, which is the value in thermal equilibrium, where the the spins of all the quasiparticles have aligned to the same spin basis. Therefore, we investigate the corresponding single-valued Boltzmann equation. Chapter 2 contains the introduction of a Boltzmann equation in one dimension, which can be used for any kind of band structure.

In Chapter 3, we investigate the first model which is a single-band model. There, we will investigate the interaction among $\uparrow$ - and $\downarrow$-spin fermions leading to thermalization of the quasiparticle momentum distribution if integrability is broken by an next-to-nearest-neighbor hopping. Away from half filling, we will see an exponentially big timescale in the inverse temperature $\beta$. At half filling, Umklapp processes relax the exponential growth of these timescales. Yet, for some initial quasiparticle momentum distributions, the timescale will still be 
exponentially large in $\beta$. We will suggest an interpretation of the properties of such quasiparticle momentum distributions.

The second model has four single-particle bands. It is an effective model to describe a one-dimensional version of a manganite material. In Chapter 4, we investigate the effects of a band structure on the thermalization rate. We will see that for large band gaps, there are quantities arising that are conserved for two-particle-interaction.

Chapter 5 summarizes the results of this work and gives an outlook on future research possibilities. The appendix includes calculations, that were omitted in the main part in order to simplify the reading flow. Nonetheless, they can be very instructive to get a deeper knowledge about the theoretical basis of the thesis. 


\section{Chapter 2}

\section{The multi-band Boltzmann equation}

The purpose of this thesis is to investigate the thermalization of a closed quantum system in the thermodynamic limit. As we explained in the introduction, this is why we use a Boltzmann-equation approach. In this chapter, we introduce the Boltzmann equation using a general Hamiltonian. In Chapters 3 and 4, we will apply this approach to the Hubbard model and the praseodymium calcium manganite chain, respectively. With the linearization scheme (shown in Sec. 2.2), we lay the groundwork to systematically describe the long-time convergence of the quasiparticle momentum distribution to the thermal one in Chapter 3 and Chapter 4. Furthermore, the linearization enables to identify quantities, which are conserved by the Boltzmann equation. The two quantities, which are conserved in every case, are treated in Sec. 2.3. We will show that they are connected to particle-number conservation and energy conservation. Moreover, the linearized Boltzmann equation includes a linear operator. The eigenvalues of the latter will turn out to be the relaxation rates. Sec. 2.4 will show that all of the respective eigenfunctions can be linked to a state-space operator, which is the physical quantity, whose expectation value relaxes with the relaxation rate given by the corresponding eigenvalue.

The last section of this chapter, Sec. 2.5, contains the proof that in certain situations the collision term of the Boltzmann equation cannot vanish. Such a situation occurs, as this section will show, if the dispersion relation is continuous and periodic.

\subsection{Setting up a Boltzmann equation for fermions}

We will investigate the relaxation of a multi-band quasiparticle momentum distribution $n_{\sigma \nu}(k, t)$. The method of our choice is a Boltzmann-equation approach. This section is concerned with setting up a multi-band Boltzmann equation of the form

$$
\dot{n}_{\sigma \nu}(k, t)=\mathcal{I}_{\text {coll }}[n]_{\sigma \nu}(k, t),
$$


with the spin $\sigma \in\{\uparrow, \downarrow\}$, the band index $\nu \in \mathbb{B}:=\left\{1,2, \ldots, N_{\mathrm{b}}\right\}$ and momentum $k$ being in the first Brillouin zone $\mathbb{K}:=\left(-\frac{1}{2}, \frac{1}{2}\right] . \mathcal{I}_{\text {coll }}$ is the collision term of the Boltzmann equation. It is an operator acting on the quasiparticle momentum distribution and describing the change of the quasiparticle momentum distribution $\dot{n}_{\sigma \nu}(k, t) \equiv \partial n_{\sigma \nu}(k, t) / \partial t$. We will derive this Boltzmann equation for fermionic Hamiltonians of the form

$$
\begin{aligned}
\hat{H} & =\hat{H}_{0}+\hat{H}_{\mathrm{int}}, \\
\hat{H}_{0} & =\int d k \sum_{\sigma \nu} \hbar \Omega_{\nu}(k) \hat{n}_{\sigma \nu}(k), \\
\hat{H}_{\mathrm{int}} & =U \int_{\mathbb{K}^{4}} d^{4} k \delta\left(\operatorname{kmod}\left(\Delta k_{\vec{k}}\right)\right) \sum_{\vec{\nu} \in \mathbb{B}^{4}} \Phi_{\vec{\nu}, \vec{k}} \hat{c}_{\uparrow \nu_{1}}^{\dagger}\left(k_{1}\right) \hat{c}_{\downarrow \nu_{2}}^{\dagger}\left(k_{2}\right) \hat{c}_{\downarrow \nu_{3}}\left(k_{3}\right) \hat{c}_{\uparrow \nu_{4}}\left(k_{4}\right) .
\end{aligned}
$$

Hereby, we introduced the fermionic creation and annihilation operators $\hat{c}_{\sigma \nu}(k)$ and $\hat{c}_{\sigma \nu}^{\dagger}(k)$, the fermionic number operators $\hat{n}_{\sigma \nu}(k)=\hat{c}_{\sigma \nu}^{\dagger}(k) \hat{c}_{\sigma \nu}(k)$, the singleparticle bands $\Omega_{\nu}(k)$, the change in total momentum $\Delta k_{\vec{k}}:=k_{1}+k_{2}-k_{3}-k_{4}$, the interaction strength $U$, and the dimensionless interaction matrix element $\Phi_{\vec{\nu}, \vec{k}}$. Lastly, we defined the function

$$
\begin{aligned}
\operatorname{kmod}: \mathbb{R} & \rightarrow \mathbb{K}=\left(-\frac{1}{2}, \frac{1}{2}\right] \\
k & \mapsto \begin{cases}\frac{1}{2} & \text { for } k \in \mathbb{Z}+\frac{1}{2} \\
\left(\left(k+\frac{1}{2}\right) \bmod 1\right)-\frac{1}{2} & \text { else. }\end{cases}
\end{aligned}
$$

It maps its parameter into the first Brillouin zone $\left(-\frac{1}{2}, \frac{1}{2}\right]$ using shifts with the reciprocal lattice vector 1 . The $\delta$-distribution containing kmod simply results from summing up Fourier functions

$$
\sum_{j} e^{i k j}=\delta(\operatorname{kmod}(k))
$$

This part of the thesis is concerned with statements valid for any number of bands and dispersion relations $\Omega_{\nu}(k)$. Both of the Hamiltonians, which we will consider later on, can be written in the form of Eq. (2.2).

In the following, we will summarize the derivations in App. B.1. The derivation of the Boltzmann equation is based on Erdös et al. (2004) and starts out with the quantum mechanical time evolution, which is known from any textbook about quantum mechanics, e.g. Basdevant and Dalibard (2002):

$$
\dot{n}_{\sigma \nu}(k, t)=\frac{\partial}{\partial t} \operatorname{tr}\left\{\hat{\rho}(t) \hat{n}_{\sigma \nu}(k)\right\}=\frac{i}{\hbar} \operatorname{tr}\left\{\hat{\rho}(t)\left[\hat{n}_{\sigma \nu}(k), \hat{H}\right]\right\},
$$

where $\hat{\rho}(t)$ is the density matrix. We introduce a shorter notation for the expectation values:

$$
\langle\hat{A}\rangle_{t}:=\operatorname{tr}\{\hat{\rho}(t) \hat{A}\}
$$

for every operator $\hat{A}$.

Using Eq. (2.5) and the time evolution of the four-point-function, we derive the collision term for the momentum distribution $n_{\sigma \nu}(k, t)$ of the quasiparticles in 
Eq. (B.49):

$$
\begin{aligned}
\mathcal{I}_{\text {coll }}[n]_{\nu_{1}}\left(k_{1}, t\right)=\frac{2 \pi U^{2}}{\hbar J} & \int_{\mathbb{K}^{3}} d k_{2} d k_{3} d k_{4} \delta\left(\operatorname{kmod}\left(\Delta k_{\vec{k}}\right)\right) \sum_{\substack{\nu_{2}, \nu_{3}, \nu_{4} \\
\in\{1, ., 4\}}} \delta\left(\Delta \Omega_{\vec{\nu}, \vec{k}}\right)\left|\Phi_{\vec{\nu}, \vec{k}}\right|^{2} \\
\times & \{\underbrace{\left[1-n_{\nu_{1}}\left(k_{1}, t\right)\right]\left[1-n_{\nu_{2}}\left(k_{2}, t\right)\right] n_{\nu_{3}}\left(k_{3}, t\right) n_{\nu_{4}}\left(k_{4}, t\right)}_{\text {gain term }} \\
& \underbrace{-n_{\nu_{1}}\left(k_{1}, t\right) n_{\nu_{2}}\left(k_{2}, t\right)\left[1-n_{\nu_{3}}\left(k_{3}, t\right)\right]\left[1-n_{\nu_{4}}\left(k_{4}, t\right)\right]}_{\text {loss term }}\} .
\end{aligned}
$$

This collision term is a low interaction limit. It consists of momentum conservation and energy conservation with the change in momentum $\Delta k_{\vec{k}}:=k_{1}+k_{2}-k_{3}-k_{4}$ and the change in energy $\Delta \Omega_{\vec{\nu}, \vec{k}}:=\Omega_{\nu_{1}}\left(k_{1}\right)+\Omega_{\nu_{2}}\left(k_{2}\right)-\Omega_{\nu_{3}}\left(k_{3}\right)-\Omega_{\nu_{4}}\left(k_{4}\right)$. The last factor is the sum of the gain and the loss term. Considering the loss term, the quasiparticle momentum distribution $n_{\nu_{1}}\left(k_{1}, t\right)$ can only become less occupied if it is not empty already, i.e. $n_{\nu_{1}}\left(k_{1}, t\right)>0$. In the classical Boltzmann equation, the factors $1-n_{\nu_{i}}\left(k_{i}, t\right)$ are missing. Here, they prevent the quasiparticle momentum distribution $n_{\nu_{1}}\left(k_{1}, t\right)$ from growing larger than 1 . The gain term leads to the fact that the quasiparticle momentum distribution $n_{\nu_{1}}\left(k_{1}, t\right)$ only increases if it is not completely full, i.e. $n_{\nu_{1}}\left(k_{1}, t\right)<1$. Thence, the factors $1-n_{\nu_{i}}\left(k_{i}, t\right)$ are attributed to the fermionic nature of the Hamiltonian.

The details of the collision term's calculation are written in App. B.1. Here, we will only point out the assumptions, which lead to the collision term.

Assumption 1: Weak interaction strength $U$. The momentum distribution $n_{\sigma \nu}(k, t)$, which will appear in the collision term, is taken in the zero interaction limit. Therefore, the Boltzmann equation describes the time evolution of the quasiparticle momentum distribution. As pointed out by Erdös et al. (2004), instead of small $U$, one can also take the low-density limit to derive a Boltzmann equation.

Assumption 2: The Boltzmann equation is assumed to be correct on times $t=\mathcal{O}\left(J / U^{2}\right)$, which are known as kinetic timescales (Fürst et al., 2013a). In combination with $U \rightarrow 0$, this leads to the energy conserving delta distribution $\delta\left(\Delta \Omega_{\vec{\nu}, \vec{k}}\right)$.

Assumption 3: We need to make an assumption about the 2-by-2 matrices

$$
\left(\left\langle\hat{c}_{\sigma \nu}^{\dagger}(k) \hat{c}_{\sigma^{\prime} \nu}(k)\right\rangle_{t}\right)_{\sigma \sigma^{\prime}} \text {. }
$$

Having derived a Boltzmann equation for these matrices, Fürst et al. (2012, $2013 b$ ) found that they become diagonal during relaxation. As an example, the thermal density matrix $e^{-\beta \hat{H}+\beta \mu \hat{N}}$ has the property (2.9), because the Hamiltonian $\hat{H}$ and the total particle number operator $\hat{N}$ both commute with the total spin operator $\frac{\hbar}{2}\left(\hat{N}_{\uparrow}-\hat{N}_{\downarrow}\right)$. Furthermore, in a discussion with Spohn (2014) it became clear, that it is unknown how to linearize their matrix-valued Boltzmann equation, because the occurring 2-by-2-matrices do not commute. However, the linearization of the Boltzmann equation is an essential part of this thesis. Therefore, we assume that the off-diagonal elements of Eq. (2.8) have already vanished, i.e.

$$
\left\langle\hat{c}_{\sigma \nu}^{\dagger}(k) \hat{c}_{\sigma^{\prime} \nu}(q)\right\rangle_{t} \propto \delta_{\sigma \sigma^{\prime}}
$$


This means that our method cannot track the diagonalization process of Eq. (2.8), but we will find only the relaxation rates of the time evolution of the quasiparticle momentum distribution $\left\langle\hat{c}_{\sigma \nu}^{\dagger}(k) \hat{c}_{\sigma \nu}(q)\right\rangle_{t}$. Furthermore, Eq. (2.9) leads to the fact that two-point-functions as in Eq. (2.9) are reduced to quasiparticle momentum distributions.

Assumption 4: We need to assume that spin-up fermions have the same quasiparticle momentum distribution like the spin-down fermions. The result is

$$
\left\langle\hat{c}_{\sigma \nu}^{\dagger}(k) \hat{c}_{\sigma^{\prime} \nu}(q)\right\rangle_{t} \propto \delta_{\sigma \sigma^{\prime}} n_{\nu}(k, t)
$$

with $n_{\nu}(k, t):=n_{\uparrow \nu}(k, t)=n_{\downarrow \nu}(k, t)$. This is not an artificial assumption, because, for example, the excitation of an electron with a photon does not depend on the spin of the electron.

Assumption 5: Spatial homogeneity. This means that we require

$$
\left\langle\hat{c}_{i, \sigma}^{\dagger} \hat{c}_{j, \sigma^{\prime}}\right\rangle_{t}=\left\langle\hat{c}_{i-j, \sigma}^{\dagger} \hat{c}_{0, \sigma^{\prime}}\right\rangle_{t},
$$

with the real-space creation and annihilation operators $\hat{c}_{i, \sigma}^{\dagger}$ and $\hat{c}_{i, \sigma^{\prime}}$, respectively. It leads to

$$
\left\langle\hat{c}_{\sigma \nu}^{\dagger}(k) \hat{c}_{\sigma^{\prime} \nu^{\prime}}(q)\right\rangle_{t} \propto \delta(k-q) .
$$

This means that two particles with different momenta are uncorrelated.

Assumption 6: Building on the last assumption, for the case of multiple bands, we have to assume that also particles in different single-particle bands are uncorrelated in the same way:

$$
\left\langle\hat{c}_{\sigma \nu}^{\dagger}(k) \hat{c}_{\sigma^{\prime} \nu}(q)\right\rangle_{t} \propto \delta_{\nu \nu^{\prime}}
$$

If one would want to relax this condition, one had to derive a Boltzmann equation for the $N_{\mathrm{b}}$-by- $N_{\mathrm{b}}$ matrices $\left[\left\langle\hat{c}_{\sigma \nu}^{\dagger}(k) \hat{c}_{\sigma \nu}(k)\right\rangle_{t}\right]_{\nu \nu^{\prime}}$. This would lead to a matrix-valued Boltzmann equation. However, as we stated in the assumption for Eq. (2.9), it is not known how to linearize this kind of Boltzmann equation. In total, Eqs. (2.9), (2.10), and (2.13) yield

$$
\left\langle\hat{c}_{\sigma \nu}^{\dagger}(k) \hat{c}_{\sigma^{\prime} \nu}(q)\right\rangle_{t} \propto \delta_{\sigma \sigma^{\prime}} \delta_{\nu \nu^{\prime}} \delta(k-q) .
$$

This is necessary to transform the four- and eight-point functions into expressions that are made up of momentum distributions $n_{\sigma \nu}(k, t)=\left\langle\hat{c}_{\sigma \nu}^{\dagger}(k) \hat{c}_{\sigma \nu}(k)\right\rangle_{t}$.

Assumption 7: Restricted quasi-freeness. Quasi-freeness means that the density matrix has the form $\exp \left(\sum_{\sigma i j} A_{\sigma i j} \hat{c}_{\sigma i}^{\dagger} \hat{c}_{\sigma j}\right)$. This results into a Wick theorem for all n-point function. The restricted quasi-freeness, however, only assumes a density matrix so that there is a Wick theorem for the four- and six-point functions. Hence, these functions are reduced to sums of products of two-point functions.

In the classical Boltzmann equation, a particle is supposed to collide just once or twice. This justifies the assumption that particles are completely uncorrelated. This assumption leads to the fact that there are no reoccurrences ${ }^{1}$ in the Boltzmann equation. It always converges to a stationary state, while the entropy is

\footnotetext{
${ }^{1}$ Reoccurrence means that the system is in the initial state again after the reoccurrence time.
} 
monotonically increasing. This is alright, as long as the times are much shorter than the reoccurrence time, which is practically always the case for macroscopic systems. The restricted quasi-freeness is the quantum-mechanical equivalent to the classical particles being completely uncorrelated. It assumes that the correlation functions can be treated as if we had a free state. This means that particles are not influenced by each other, as long as they do not collide.

Next, we will get rid of the kmod function in the momentum conservation. All the functions in the integrand are 1-periodic in all the $k_{j}$, therefore we can trade it for a larger integration space. Specifically, we have to enlarge the integration interval of one momentum. We choose this momentum to be $k_{4}$ and obtain

$$
\int_{\mathbb{K}^{3}} d k_{2} d k_{3} d k_{4} \delta\left(\operatorname{kmod}\left(\Delta k_{\vec{k}}\right)\right) \rightarrow \int_{\mathbb{K}^{2} \times \mathbb{R}} d k_{2} d k_{3} d k_{4} \delta\left(\Delta k_{\vec{k}}\right) .
$$

This will be very useful later on in the calculation of the interaction channels. It basically means that for any $\left(k_{1}, k_{2}, k_{3}\right) \in \mathbb{K}^{3}$ one gets exactly $k_{4}=k_{1}+k_{2}-k_{3}$. If $k_{4}$ is outside the first Brillouin zone it simply means that the process is an Umklapp process. This does not affect the other parts of the integrand, because it is 1 -periodic in all $k_{j}$.

The Boltzmann equation now reads

$$
\begin{aligned}
\mathcal{I}_{\text {coll }}[n]_{\nu_{1}}\left(k_{1}, t\right)=\frac{2 \pi U^{2}}{\hbar J} \int_{\mathbb{K}^{2} \times \mathbb{R}} d k_{2} d k_{3} d k_{4} \delta\left(\Delta k_{\vec{k}}\right) \sum_{\nu_{2,3,4} \in \mathbb{B}} \delta\left(\Delta \Omega_{\vec{\nu}, \vec{k}}\right)\left|\Phi_{\vec{\nu}, \vec{k}}\right|^{2} \\
\times\left\{\left[1-n_{\nu_{1}}\left(k_{1}, t\right)\right]\left[1-n_{\nu_{2}}\left(k_{2}, t\right)\right] n_{\nu_{3}}\left(k_{3}, t\right) n_{\nu_{4}}\left(k_{4}, t\right)\right. \\
\left.\quad-n_{\nu_{1}}\left(k_{1}, t\right) n_{\nu_{2}}\left(k_{2}, t\right)\left[1-n_{\nu_{3}}\left(k_{3}, t\right)\right]\left[1-n_{\nu_{4}}\left(k_{4}, t\right)\right]\right\} .
\end{aligned}
$$

It describes the time evolution of the quasiparticle momentum distribution. This is confirmed by derivations using Green functions, see Rammer and Smith (1986); Kamenev and Levchenko (2009). Note that momentum conservation leads to $\left|k_{4}\right|<\frac{3}{2}$.

For any kind of dispersion relation, the last factor in Eq. (2.16) vanishes if the distribution is equal to the Fermi-Dirac distribution

$$
f_{\nu}(k)=\frac{1}{\exp \left[\beta\left(\Omega_{\nu}(k)-\mu\right)\right]+1},
$$

see App. C.7. Therefore, $f_{\nu}(k)$ is a fixed point of the Boltzmann equation. Furthermore, it is a stable fixed point, because the linearization around this distribution leads to a positive semidefinite linear operator $\hat{\mathcal{L}}$, which we will discuss in Sec. 2.2.

It is important to note that depending on the dispersion relation, there may be more fixed points of the Boltzmann equation. For example, the cosine dispersion relation has many non-thermal stationary states, see Sec. 3.2.1. 


\subsection{Linearization of the Boltzmann equation}

In order to find the relaxation rates of the quasiparticle momentum distribution, we will linearize the Boltzmann equation around its thermal distribution, the Fermi-Dirac distribution $f_{n}(k)$ defined in Eq. (2.17). The linearization scheme we use is described in the book by Haug and Jauho (1996) and presented in this section. Its advantage of the linearized Boltzmann equation is that it reveals, which parts of the momentum distributions relax how fast. Without the possibility of linearization, one would have to evolve different initial momentum distributions in time, and try to extract the longest relaxation time.

It is important to note that it is an open question (Spohn, 2014) how to linearize the full matrix-valued Boltzmann-equation derived by Fürst et al. (2012, 2013a,b). The 2-by-2 matrices involved in this case make the problem very difficult, because they do not commutate in general.

The first step is defining the perturbation $\phi_{\nu}(k, t)$ of the quasiparticle momentum distribution:

$$
n_{\nu}(k, t)=\frac{1}{1+\exp \left[\beta\left(\Omega_{\nu}(k)-\mu\right)-\phi_{\nu}(k, t)\right]} .
$$

For any real-valued functions $\phi_{\nu}(k, t)$, the resulting momentum distribution $n_{\nu}(k, t)$ has values between 0 and 1 . Thus, $n_{\nu}(k, t)$ is a valid quasiparticle momentum distribution for fermions.

The linearization of Eq. (2.18) yields

$$
n_{\nu}(k, t)=f_{\nu}(k)+f_{\nu}(k)\left[1-f_{\nu}(k)\right] \phi_{\nu}(k, t)+\mathcal{O}\left(\phi^{2}\right) .
$$

We plug this into the collision term, Eq. (2.16), and then linearize the result. The linearization is shown in detail in App. B.2. The resulting linearized Boltzmann equation takes the form

$$
\dot{\phi}_{\nu}(k, t)=-\hat{\mathcal{L}}[\phi]_{\nu}(k, t)
$$

with the linear operator

$$
\begin{array}{r}
\hat{\mathcal{L}}[\phi]\left(k_{1}, t\right)=\frac{2 \pi U^{2}}{\hbar^{2}} \int_{\mathbb{K}^{2} \times \mathbb{R}} d k_{2} d k_{3} d k_{4} \sum_{\substack{\nu_{2}, \nu_{3}, \nu_{4} \\
\in\{1, . ., 4\}}} \frac{\left[1-f_{\nu_{1}}\left(k_{1}\right)\right]\left[1-f_{\nu_{2}}\left(k_{2}\right)\right] f_{\nu_{3}}\left(k_{3}\right) f_{\nu_{4}}\left(k_{4}\right)}{f_{\nu_{1}}\left(k_{1}\right)\left[1-f_{\nu_{1}}\left(k_{1}\right)\right]} \\
\times \delta\left(\Delta k_{\vec{k}}\right) \delta\left(\Delta \Omega_{\vec{\nu}, \vec{k}}\right)\left|\Phi_{\vec{\nu}, \vec{k}}\right|^{2}\left[\phi_{\nu_{1}}\left(k_{1}, t\right)+\phi_{\nu_{2}}\left(k_{2}, t\right)-\phi_{\nu_{3}}\left(k_{3}, t\right)-\phi_{\nu_{4}}\left(k_{4}, t\right)\right] .
\end{array}
$$

This formula is Eq. (B.62) from the appendix. In order to formally solve the linear rate equation in Eq. (2.20), we will introduce the eigenfunctions of $\hat{\mathcal{L}}$, which is possible, because $\hat{\mathcal{L}}$ is hermitian in the scalar product (see Eq. (B.64))

$$
\langle\phi, \psi\rangle_{\mathrm{F}}:=\int d k \sum_{n} \phi_{\nu}(k) f_{\nu}(k)\left[1-f_{\nu}(k)\right] \psi_{\nu}(k), .
$$

Therefore, its eigenvalues $\lambda_{n}$ are real-valued and the eigenfunctions $\chi_{n}(k)$ are orthogonal in this scalar product. The scalar product induces the norm

$$
\|\phi\|_{\mathrm{F}}:=\sqrt{\langle\phi, \phi\rangle_{\mathrm{F}}}
$$


It is important to note that for a small final temperature $T$, the scalar product is of $\mathcal{O}(T)$, which is shown by a Sommerfeld expansion, see App. B.4. For $T=0$, the scalar product vanishes, therefore it cannot induce a norm. However, even $\|\cdot\|_{\mathrm{F}} / T=\mathcal{O}(1)$ is not a norm for temperature zero. The reason is the factor $f_{\nu}(k)\left[1-f_{\nu}(k)\right] / T=f_{\nu}^{\prime}(k) / \Omega_{\nu}^{\prime}(k)$ in the scalar product $\langle\phi, \psi\rangle_{\mathrm{F}} / T$. The derivative $f_{\nu}^{\prime}(k)$ becomes increasingly narrow in the limit $T \rightarrow 0$. Finally, at $T=0$, it becomes proportional to $\delta\left(k+k_{\mathrm{F}}\right)-\delta\left(k-k_{\mathrm{F}}\right)$. Hence, in this limit, the scalar product only takes into account the values of $\phi$ and $\psi$ at the two Fermi momenta $\pm k_{\mathrm{F}}$. Therefore, $\lim _{T \rightarrow 0}\|\cdot\|_{\mathrm{F}} / T$ is only a semi-norm. However, we are not interested in a final temperature of zero, because this implies that the initial momentum distribution has already been the thermal distribution with temperature zero. Thus, the scalar product induces a norm for the cases we are considering.

The scalar product and the induced norm lead to the Hilbert space

$$
\left\{g \in L^{2}(\mathbb{R}, \mathbb{R}) \mid g(k+1)=g(k) \forall k\right\} .
$$

The eigenfunctions $\chi_{\nu}^{(j)}(k)$ of $\hat{\mathcal{L}}$ span this space. Thus, we can represent the perturbation $\phi_{\nu}(k)$ with the eigenfunctions $\chi_{\nu}^{(j)}(k)$ :

$$
\phi_{\nu}(k, t)=\sum_{j} A_{j}(t) \chi_{\nu}^{(j)}(k)
$$

The linear rate Eq. (2.20) leads to

$$
A_{j}(t)=A_{j}(0) e^{-\lambda_{j} t}
$$

and therefore

$$
\phi_{\nu}(k, t)=\sum_{j} A_{j}(0) e^{-\lambda_{j} t} \chi_{\nu}^{(j)}(k) .
$$

This is Eq. (B.76) from the appendix. The coefficient

$$
A_{j}(0)=\frac{\left\langle\chi^{(j)}, \phi\right\rangle_{\mathrm{F}}}{\left\|\chi^{(j)}\right\|_{\mathrm{F}}^{2}}
$$

is the initial contribution of the $j^{\text {th }}$ eigenfunction. Eq. (2.26) shows that a contribution $A_{j}(t)$ decays exponentially with the rate $\lambda_{j}$. Thence, the eigenvalues $\lambda_{j}$ of the linear operator $\hat{\mathcal{L}}$ are the relaxation rates.

Since $\hat{\mathcal{L}}$ is positive semidefinite, the eigenvalues are positive. Thus, there is no exponential growth, which would lead away from the thermal distribution $f_{\nu}(k)$.

Furthermore, it is important to note that $\hat{\mathcal{L}} / U^{2}$ is independent of $U$. It is obvious that this leads to the following two consequences. Firstly, every eigenvalue $\lambda_{j}$ is proportional to $U^{2}$. Secondly, the eigenfunctions $\chi_{\nu}^{(j)}(k)$ do not depend on $U$ at all. This means that the eigenfunctions always stay in the $U \rightarrow 0$ limit. 


\subsection{Conserved contributions}

The eigenvalues $\lambda_{j}$, which are zero, correspond to constant contributions, i.e. $A_{j}(t)=A_{j}(0)$ for all times $t$. Independent of the form of the dispersion relation $\Omega_{\nu}(k)$, there are always two eigenvalues that are zero, the eigenvalue $\lambda_{1}=$ 0 corresponding to the constant function and $\lambda_{2}=0$ corresponding to the dispersion relation, i.e.:

$$
\begin{aligned}
& \chi_{\nu}^{(1)}(k)=\text { const } \\
& \chi_{\nu}^{(2)}(k)=\Omega_{\nu}(k) .
\end{aligned}
$$

The formal reason for them to have an eigenvalue of zero is that the factor $\phi_{\nu_{1}}\left(k_{1}, t\right)+\phi_{\nu_{2}}\left(k_{2}, t\right)-\phi_{\nu_{3}}\left(k_{3}, t\right)-\phi_{\nu_{4}}\left(k_{4}, t\right)$ in the operator $\hat{\mathcal{L}}$ (see Eq. $\left.(2.21)\right)$ vanishes for both of them:

$$
\begin{aligned}
& \chi_{\nu_{1}}^{(1)}\left(k_{1}\right)+\chi_{\nu_{2}}^{(1)}\left(k_{2}\right)-\chi_{\nu_{3}}^{(1)}\left(k_{3}\right)-\chi_{\nu_{4}}^{(1)}\left(k_{4}\right)=\mathrm{const} \cdot(1+1-1-1)=0, \\
& {\left[\chi_{\nu_{1}}^{(2)}\left(k_{1}\right)+\chi_{\nu_{2}}^{(2)}\left(k_{2}\right)-\chi_{\nu_{3}}^{(2)}\left(k_{3}\right)-\chi_{\nu_{4}}^{(2)}\left(k_{4}\right)\right] \delta\left(\Delta \Omega_{\vec{\nu}, \vec{k}}\right)=\Delta \Omega_{\vec{\nu}, \vec{k}} \delta\left(\Delta \Omega_{\vec{\nu}, \vec{k}}\right)=0 .}
\end{aligned}
$$

In order to find the physical reason, we have to see what happens if there are contributions of these two eigenfunctions $\chi_{\nu}^{(1,2)}(k)$ in the perturbation $\phi_{\nu}(k, t)$. Therefore, we consider the definition $n_{\nu}(k, t)=1 /\left\{1+\exp \left[\beta\left(\Omega_{\nu}(k)-\mu\right)-\right.\right.$ $\left.\left.\phi_{\nu}(k, t)\right]\right\}$ of the perturbation, see Eq. (2.18). Plugging Eq. (2.27) into this equation, the argument of the exponential becomes

$$
\begin{aligned}
& \beta\left(\Omega_{\nu}(k)-\mu\right)-\sum_{j} A_{j}(0) e^{-\lambda_{j} t} \chi_{\nu}^{(j)}(k) \\
& =\left(\beta-A_{1}(0)\right) \Omega_{\nu}(k)-\left(\beta \mu+A_{2}(0)\right)-\sum_{j \geqslant 3} A_{j}(0) e^{-\lambda_{j} t} \chi_{\nu}^{(j)}(k) .
\end{aligned}
$$

If $\phi_{\nu}(k)$ has a contribution of either of these eigenfunctions, the inverse temperature $\beta$ and the chemical potential $\mu$ may become modified. Thus, the time evolution leads to another Fermi-Dirac distribution consisting of a different final temperature $\beta_{\text {final }}$ and a different chemical potential $\mu_{\text {final }}$ :

$$
\begin{aligned}
\beta_{\text {final }} & =\beta-A_{2}(0) \\
\beta_{\text {final }} \mu_{\text {final }} & =\beta \mu-A_{1}(0) \Longrightarrow \mu_{\text {final }}=\frac{\beta \mu-A_{1}(0)}{\beta-A_{2}(0)}
\end{aligned}
$$

Hence, $\beta$ and $\mu$ are not the true final temperature and chemical potential, respectively, but $\beta_{\text {final }}$ and $\mu_{\text {final }}$ are. If we redefine $\beta_{\text {final }} \rightarrow \beta$ and $\mu_{\text {final }} \rightarrow \mu$, the contributions $A_{1}(0)$ and $A_{2}(0)$ vanish. Consequently, for every quasiparticle momentum distribution $n_{\nu}(k, t)$ the perturbation $\phi_{\nu}(k)$ can be defined in such a way that $A_{1}(0)=A_{2}(0)=0$. Therefore, any contribution from the first two eigenfunctions $\chi_{\nu}^{(1,2)}(k)$ can be avoided.

Furthermore, the eigenfunction $\chi_{\nu}^{(1)}(k)=$ const seems to be connected to the total particle number, because it modifies $\beta \mu$ and the eigenfunction $\chi_{\nu}^{(2)}(k)=$ $\Omega_{\nu}(k)$ is apparently related to the total energy, because it modifies the inverse temperature $\beta$. These two connections become clearer in Sec. 2.4.

It is important to note that $\phi_{\nu}(k)=k$, which corresponds to the total momentum, is not an eigenfunction of $\hat{\mathcal{L}}$, since Umklapp processes are allowed. 


\subsection{State-space operators corresponding to eigen- functions of the linearized Boltzmann opera- tor}

In order to understand the physical meaning of the eigenfunctions of $\hat{\mathcal{L}}$, we make a connection to the state space, i.e. the space of the quantum-mechanical states. Therefore, we define the operator

$$
\hat{\Phi}[\psi]:=\int d k \sum_{\nu} \psi_{\nu}(k) \hat{n}_{\nu}(k),
$$

which maps the function $\psi_{\nu}(k)$ to an operator in state space. These mappings are motivated by the connection of the first two eigenfunctions $\chi_{\nu}^{(1,2)}(k)$ (see Eq. (2.29)) to the total particle number and the total energy, see Sec. 2.3. The corresponding operators are

$$
\hat{N}_{\text {tot }}=\int d k \sum_{\nu} \hat{n}_{\nu}(k)=\hat{\Phi}\left[\chi^{(1)}\right]
$$

and

$$
\hat{H}=\int d k \sum_{\nu} \Omega_{\nu}(k) \hat{n}_{\nu}(k)+\mathcal{O}(U)=\hat{\Phi}\left[\chi^{(2)}\right]+\mathcal{O}(U) .
$$

In Sec. 2.3, we learned that the eigenfunctions always stay in the $U \rightarrow 0$ limit. So does the eigenfunction $\chi_{\nu}^{(2)}(k)$, which is related to the energy. This is the reason why the operator $\hat{\Phi}\left[\chi^{(2)}\right]$ is only the $U \rightarrow 0$ limit of the total energy, i.e. the kinetic energy, see Eq. (2.35).

So far, we only defined operators. Now, we will explain, why this definition is physically reasonable for every eigenfunction of $\hat{\mathcal{L}}$. This is done by the expectation values of the operators.

The expectation values are

$$
\begin{aligned}
\langle\hat{\Phi}[\psi]\rangle_{t} & =\int d k \sum_{\nu} \psi_{\nu}(k) n_{\nu}(k, t) \\
\stackrel{(2.19)}{=} \int d k \sum_{\nu} \psi_{\nu}(k)\left[f_{\nu}(k)+f_{\nu}(k)\left(1-f_{\nu}(k)\right) \phi_{\nu}(k)\right]+\mathcal{O}\left(\phi^{2}\right) & \\
& =\int d k \sum_{\nu} \psi_{\nu}(k) f_{\nu}(k)+\langle\psi, \phi\rangle_{\mathrm{F}}+\mathcal{O}\left(\phi^{2}\right) \\
& =\underbrace{\int d k \sum_{\nu} \psi_{\nu}(k) f_{\nu}(k)}_{=\text {const }}+\sum_{j} A_{j}(0) e^{-\lambda_{j} t}\left\langle\psi, \chi^{(j)}\right\rangle_{\mathrm{F}}+\mathcal{O}\left(\phi^{2}\right) .
\end{aligned}
$$

In the case of $\psi$ being antisymmetric around $k=0$, the constant contribution is zero.

Now, we plug the eigenfunctions into $\hat{\Phi}$ and get the expectation value

$$
\left\langle\hat{\Phi}\left[\chi^{(j)}\right]\right\rangle_{t}=\underbrace{\int d k \chi_{\nu}^{(j)}(k) f_{\nu}(k)}_{=\text {const }}+A_{j}(0) e^{-\lambda_{j} t} .
$$


We find that the time evolution of the expectation value of $\hat{\Phi}\left[\chi^{(j)}\right]$ resembles the time evolution of perturbation $\phi_{\nu}(k)$ of the quasiparticle momentum distribution $n_{\nu}(k, t)$. Thus, the operators $\hat{\Phi}\left[\chi^{(j)}\right]$ help us to understand the physical meaning of the eigenfunctions.

Further examples of operators in the form of Eq. (2.33) are the total momentum operator, the total particle current, the total energy current, and the total heat current:

$$
\begin{aligned}
\hat{K} & =\int d k \sum_{\nu} k \hat{n}_{\nu}(k)=\hat{\Phi}[\mathrm{id}] \\
\hat{J}^{N} & =\frac{J}{\pi \hbar} \int d k \sum_{\nu} \Omega_{\nu}^{\prime}(k) \hat{n}(k)=\hat{\Phi}\left[j^{N}\right] \\
\hat{J}^{E} & =\frac{4 J^{2}}{\pi \hbar} \int d k \sum_{\nu} \Omega_{\nu}^{\prime}(k) \Omega_{\nu}(k) \hat{n}(k)+\mathcal{O}(U)=\hat{\Phi}\left[j^{E}\right]+\mathcal{O}(U) \\
\hat{J}^{Q} & =\hat{J}^{E}-\mu \hat{J}^{N}=\hat{\Phi}\left[j^{Q}\right]+\mathcal{O}(U),
\end{aligned}
$$

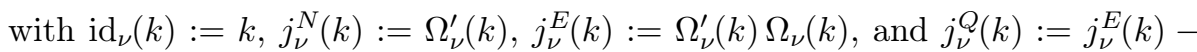
$\mu j_{\nu}^{N}(k)$. The currents are derived for one band in App. C.5. It is important to note that the Boltzmann equation can only cover the zeroth order in interaction. For the energy current, this means that the $U$-term cannot be measured. However, if $U$ is small enough, this part is negligible. In the discussion of the Hubbard model in Chapter 3 the operators in Eq. (2.38) will play an important role in identifying the physical meaning of the eigenfunction, which belongs to the smallest relaxation rate, see Sec. 3.5.2.

\subsection{Interaction channels}

In order to calculate the collision term $\mathcal{I}_{\text {coll }}$ (see Eq. (2.16)) or the linearized Boltzmann operator $\hat{\mathcal{L}}$ (see Eq. (2.21)), we have to perform the integrations over $k_{2}, k_{3}$, and $k_{4}$. These integrations are restricted by $\delta\left(k_{1}+k_{2}-k_{3}-k_{4}\right)$ and $\delta\left(\Omega_{\nu_{1}}\left(k_{1}\right)+\Omega_{\nu_{2}}\left(k_{2}\right)-\Omega_{\nu_{3}}\left(k_{3}\right)-\Omega_{\nu_{4}}\left(k_{4}\right)\right)$. The two $\delta$-distributions are momentum conservation and energy conservation, respectively. In order to perform the integrals, we have to know the possible combinations of $k_{1}, \ldots, k_{4}$, which obey these two conservation laws. We call these combinations "interaction channels".

There are two kinds of channels, which we denote as trivial interaction channels and non-trivial interaction channels. Both are valid interaction channels. However, trivial channels are all the channels, which do not contribute to the collision term $\mathcal{I}_{\text {coll }}$. Since $\hat{\mathcal{L}}$ is the linearization of $\mathcal{I}_{\text {coll }}$ and the trivial channels do not contribute for all quasiparticle momentum distributions, the trivial channels do not contribute to $\hat{\mathcal{L}}$ either. Only non-trivial interaction channels can make $\mathcal{I}_{\text {coll }}$ and $\hat{\mathcal{L}}$ become non-zero.

We will prove in Sec. 2.5.2 that a continuous periodic dispersion relation always lead to non-trivial channels in the Boltzmann equation. This means that in these cases the Boltzmann equation is never zero. However, it does not mean that such Boltzmann equations always lead to the thermalization of the momentum distribution, which they describe. 


\subsubsection{Trivial interaction channels and their elimination from the energy conservation}

The formal definition of a trivial channel is that

$$
\begin{aligned}
& n_{\nu_{1}}\left(k_{1}, t\right) n_{\nu_{2}}\left(k_{2}, t\right)\left[1-n_{\nu_{3}}\left(k_{3}, t\right)\right]\left[1-n_{\nu_{4}}\left(k_{4}, t\right)\right] \\
& \quad-\left[1-n_{\nu_{1}}\left(k_{1}, t\right)\right]\left[1-n_{\nu_{2}}\left(k_{2}, t\right)\right] n_{\nu_{3}}\left(k_{3}, t\right) n_{\nu_{4}}\left(k_{4}, t\right)=0
\end{aligned}
$$

for every quasiparticle momentum distribution $n_{\nu}(k, t)$. Because the perturbation $\phi_{\nu}(k, t)$ is defined by $n_{\nu}(k, t)$ (see Eq. (2.18)), Eq. (2.39) is also valid for every $\phi_{\nu}(k, t)$. Thus, when Eq. (2.39) is linearized, every coefficient has to vanish, especially the coefficient of the linear term:

$$
\begin{aligned}
f_{\nu_{1}}\left(k_{1}\right) & f_{\nu_{2}}\left(k_{2}\right)\left[1-f_{\nu_{3}}\left(k_{3}\right)\right]\left[1-f_{\nu_{4}}\left(k_{4}\right)\right] \\
& \times\left[\phi_{\nu_{1}}\left(k_{1}, t\right)+\phi_{\nu_{2}}\left(k_{2}, t\right)-\phi_{\nu_{3}}\left(k_{3}, t\right)-\phi_{\nu_{4}}\left(k_{4}, t\right)\right]=0 .
\end{aligned}
$$

The prefactor containing the Fermi-Dirac functions is positive, therefore we have

$$
\phi_{\nu_{1}}\left(k_{1}, t\right)+\phi_{\nu_{2}}\left(k_{2}, t\right)-\phi_{\nu_{3}}\left(k_{3}, t\right)-\phi_{\nu_{4}}\left(k_{4}, t\right)=0 \text {. }
$$

Let us consider an example for a trivial channel. For intra-band interactions $\left(\nu=\nu_{1}=\nu_{2}=\nu_{3}=\nu_{4}\right)$, there are trivial solutions to momentum and energy conservation, for instance $k_{1}=k_{3} \wedge k_{2}=k_{4}$. These solutions are momentum exchanges and do not change the quasiparticle momentum distribution, i.e. they do not contribute to $\mathcal{I}_{\text {coll }}$. They are trivial interaction channels, because

$$
\begin{aligned}
& n_{\nu}\left(k_{1}, t\right) n_{\nu}\left(k_{2}, t\right)\left[1-n_{\nu}\left(k_{1}, t\right)\right]\left[1-n_{\nu}\left(k_{2}, t\right)\right] \\
& \quad-\left[1-n_{\nu}\left(k_{1}, t\right)\right]\left[1-n_{\nu}\left(k_{2}, t\right)\right] n_{\nu}\left(k_{1}, t\right) n_{\nu}\left(k_{2}, t\right)=0 .
\end{aligned}
$$

Moreover, one also has

$$
\phi_{\nu}\left(k_{1}, t\right)+\phi_{\nu}\left(k_{2}, t\right)-\phi_{\nu}\left(k_{1}, t\right)-\phi_{\nu}\left(k_{2}, t\right)=0
$$

When we state the general cases of trivial channels, we do not restrict the $k_{j}$ to a certain Brillouin zone. We use a description where $k_{1}, k_{2}, k_{3} \in \mathbb{Z}$ and $k_{4}=k_{1}+k_{2}-k_{3}+m$, for any $m \in \mathbb{Z}$.

In general, there are trivial interaction channels if $\nu_{3}=\nu_{1} \wedge \nu_{4}=\nu_{2}$, or $\nu_{3}=$ $\nu_{2} \wedge \nu_{4}=\nu_{1}$. In the first case, $\nu_{3}=\nu_{1} \wedge \nu_{4}=\nu_{2}$, there is a trivial channel if there are $m, n \in \mathbb{Z}$ so that $k_{3}=k_{1}+n \wedge k_{4}=k_{2}+m$. This is equivalent to the equality

$$
\sin \left(\pi\left(k_{3}-k_{1}\right)\right)=0 .
$$

Similarly, the second case, where $\nu_{3}=\nu_{2} \wedge \nu_{4}=\nu_{1}$, is described by

$$
\sin \left(\pi\left(k_{3}-k_{2}\right)\right)=0
$$

For intra-band interactions, both cases are applicable. Then, we have

$$
\sin \left(\pi\left(k_{3}-k_{1}\right)\right) \sin \left(\pi\left(k_{3}-k_{2}\right)\right)=0 .
$$


The product of the two sinuses can be written as $\frac{1}{2}\left[\cos \left(\pi\left(2 k_{3}-k_{1}-k_{2}\right)\right)-\right.$ $\left.\cos \left(\pi\left(-k_{1}+k_{2}\right)\right)\right]$. Thus, Eq. (2.46) is equivalent to

$$
\cos \left[\pi\left(k_{1}-k_{2}\right)\right]=\cos \left[\pi\left(k_{3}-k_{4}\right)\right]
$$

which also holds for a more general quasi momentum conservation $k_{4}=k_{1}+$ $k_{2}-k_{3}+n, n \in \mathbb{Z}$.

The trivial interaction channels can be omitted in numerics to save CPU time. However, it turns out that it takes a lot of effort to do so. For the Hubbard model, there is an equation that describes only the non-trivial channels, see Eq. (3.27). This simplifies the numerical implementation in this case.

For other band structures like the one of the manganite chain in Eq. (4.38), the trivial channels can be eliminated using a division by $\sin \left(\pi\left(k_{3}-k_{1}\right)\right), \sin \left(\pi\left(k_{3}-\right.\right.$ $\left.k_{2}\right)$ ), or both. As an example, we consider $\nu_{3}=\nu_{1} \wedge \nu_{4}=\nu_{2}$. There, the nontrivial solutions are described by

$$
\frac{\Delta \Omega_{\nu_{1} \nu_{2} \nu_{1} \nu_{2}}(\vec{k})}{\sin \left(\pi\left(k_{3}-k_{1}\right)\right)}=\frac{\Omega_{\nu_{1}}\left(k_{1}\right)-\Omega_{\nu_{1}}\left(k_{3}\right)+\Omega_{\nu_{2}}\left(k_{2}\right)-\Omega_{\nu_{2}}\left(k_{2}+k_{1}-k_{3}\right)}{\sin \left(\pi\left(k_{3}-k_{1}\right)\right)} .
$$

For a very small $y:=k_{3}-k_{1}$, one can get into trouble when performing numerical calculations, because both the enumerator and the denominator go to zero. For a small $y$, we perform a Taylor expansion in $\hat{s}_{y}:=\sin (\pi y)$. For every $y \in \mathbb{R}$, we find that

$$
\Omega_{\nu}(x+\arctan [\tan (\pi y)] / \pi)=\Omega_{\nu}(x+\operatorname{kmod}(y))=\Omega_{\nu}(x+y) .
$$

It is important to note, that we do not use $\arcsin [\sin (\pi y)] / \pi$, because it gives the wrong sign in the second Brillouin zone. Now, we can expand

$$
\begin{aligned}
& \Omega_{\nu}\left(x+\arctan \left[\hat{t}_{y}\right] / \pi\right) \\
& =\Omega_{\nu}(x)+\Omega_{\nu}^{\prime}(x) \frac{\hat{t}_{y}}{\pi}+\Omega_{\nu}^{\prime \prime}(x) \frac{\hat{t}_{y}^{2}}{2 \pi^{2}}+\left[-\frac{\Omega_{\nu}^{\prime}(x)}{4 \pi}+\frac{\Omega_{\nu}^{\prime \prime \prime}(x)}{6 \pi^{3}}\right] \hat{t}_{y}^{3}+\mathcal{O}\left(\hat{t}_{y}^{4}\right),
\end{aligned}
$$

with $\hat{t}_{y}:=\tan (\pi y)$. Expanding $\Omega_{\nu_{1}}\left(k_{3}\right)$ and $\Omega_{\nu_{2}}\left(k_{2}+k_{1}-k_{3}\right)$ in this manner, we can bring Eq. (2.48) in a form, which can be numerically evaluated for small $\operatorname{kmod}(y):$

$$
\frac{\Delta \Omega_{\nu_{1} \nu_{2} \nu_{1} \nu_{2}}(\vec{k})}{\hat{s}_{y}}=\left[-\frac{\Omega_{\nu}^{\prime}\left(k_{1}\right)-\Omega_{\nu}^{\prime}\left(k_{2}\right)}{\pi \hat{c}_{y}}-\frac{\Omega_{\nu}^{\prime \prime}\left(k_{1}\right)+\Omega_{\nu}^{\prime \prime}\left(k_{2}\right)}{2 \pi^{2} \hat{c}_{y}^{2}} \hat{s}_{y}\right]+\mathcal{O}\left(\hat{s}_{y}^{2}\right)
$$

with $\hat{c}_{y}:=\cos (\pi y)$ and $y=k_{3}-k_{1}$. To find only the non-trivial channels and keep up a high precision, the points where trivial and non-trivial channels meet have to be treated very carefully.

\subsubsection{Existence of non-trivial interaction channels}

The interesting channels are those interaction channels, which contribute to the collision term. We denote these channels as non-trivial interaction channels. 


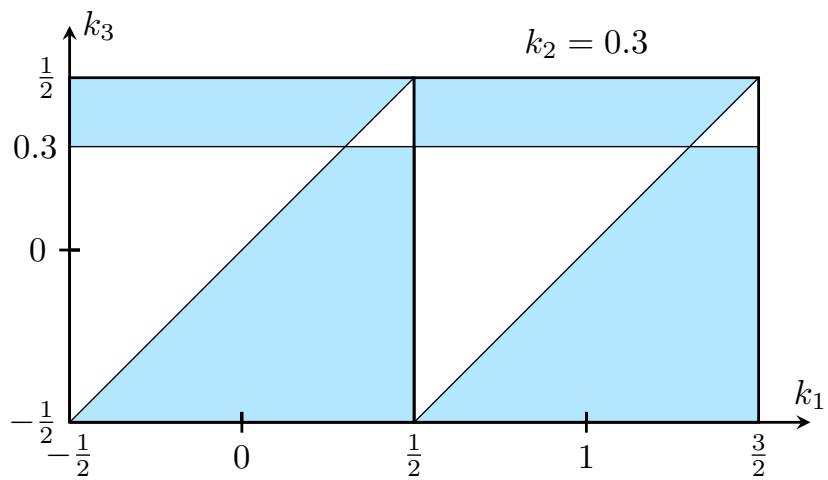

Figure 2.1: For the proof of the existence of non-trivial interaction channels: For $k_{2}=0.3$, we schematically plot $\operatorname{sgn}(\Delta E)$ as a function of $k_{1}$ and $k_{3} . \square$ denotes one sign and $\square$ the other. The trivial channels are the vertical line $k_{3}=k_{2}$ and the diagonal line $k_{3}=k_{1}$. Since $\Delta E$ is periodic in all $k_{j}$, there must be an additional sign change, which is at the Brillouin-zone in this figure.

For inter-band interactions, we prove below that such channels exist (see also Biebl and Kehrein (2016)).

The change of energy for this single band with index $\nu$ is

$$
\Delta E:=\Delta \Omega_{\nu, \nu, \nu, \nu}(\vec{k})=\Omega_{\nu}\left(k_{1}\right)+\Omega_{\nu}\left(k_{2}\right)-\Omega_{\nu}\left(k_{3}\right)-\Omega_{\nu}\left(k_{1}+k_{2}-k_{3}\right) .
$$

The proof is visualized in Fig. 2.1. There, we plotted the $\operatorname{sign}^{2}$ of the energy change $\operatorname{sgn}(\Delta E)$ as a function of $k_{1}$ and $k_{3}$ for a fixed $k_{2}=0.3$. When $\operatorname{sgn}(\Delta E)$ is zero, we have a possible two-particle interaction channel because of the continuity of the dispersion relation $\Omega_{\nu}(k)$. Beside the first Brillouin zone of $k_{1}$ and $k_{3}$, we plotted the region $\left(k_{1}, k_{3}\right) \in\left[\frac{1}{2}, \frac{3}{2}\right] \times\left[-\frac{1}{2}, \frac{1}{2}\right]$. This region looks exactly like the first Brillouin zone due to the 1-periodicity of $\Delta E$ in every $k_{j}$, which is inherited from the 1-periodicity of $\Omega_{\nu}(k)$.

If there is no non-trivial channel within the first Brillouin zone, the proof is done. Otherwise, the situation is the same as in Fig. 2.1, which means that there is a non-trivial channel at the border where $k_{1}=\frac{1}{2}$. So in fact, there is a non-trivial channel, which proves our claim.

Of course, there is the possibility that the trivial channels could be double zeros, i.e. there is a zero, but no sign change. If only one of the trivial channels is a double zero, then there will still be a sign change on at least one of the Brillouin-zone boundaries.

For the last case, we assume that both trivial interaction channels are double zeros of $\Delta E$. This leads to the fact that there is no sign change at the trivial interaction channels. Thus, if there is no further channel within the first Brillouin

${ }^{2}$ The sign function is defined as

$$
\operatorname{sgn}(x)=\left\{\begin{aligned}
1 & \text { for } x>0 \\
0 & \text { for } x=0 \\
-1 & \text { else. }
\end{aligned}\right.
$$


zone, then $\Delta E$ has exactly one sign, w.l.o.g.

$$
\Delta E \geqslant 0 \forall k_{1,2,3}, k_{4}=k_{1}+k_{2}-k_{3} .
$$

Now, we interchange $k_{1}$ with $k_{4}$ and $k_{2}$ with $k_{3}$. Momentum is still conserved, but the energy change has gained a minus sign. Hence, we have

$$
\Delta E \leqslant 0 \forall k_{1,2,3}, k_{4}=k_{1}+k_{2}-k_{3} .
$$

The result is that $\Delta E$ is zero everywhere. But this means that the dispersion relation is a constant, i.e. the band is completely flat. The constant dispersion relation has a solution for every combination of momenta, as long as momentum is conserved. Thus also in this case there are non-trivial interaction channels.

It is important to note, however, that for the case of $\Delta E \equiv 0$ the Boltzmann equation diverges, which rules out the use of the constant dispersion relation. Furthermore, the existence of non-trivial channels does not necessarily lead to thermalization within two-particle scattering, e.g. in integrable systems like the standard 1d-FHM (Fürst et al., 2012). However, we expect thermalization to happen in most cases, like the integrability broken one-dimensional Hubbard model (Fürst et al., 2013b). 


\section{Chapter 3}

\section{Relaxation rates in the Hubbard model with next-to-nearest-neighbor hopping}

In this chapter, we consider the Hubbard model with a next-to-nearest-neighbor hopping term and try to find the relaxation rates of the quasiparticle momentum distribution. In the first section, we introduce the model. Then, in Sec. 3.2, we set up the Boltzmann equation and introduce the perturbation $\phi(k)$, which linearizes it. This is needed to find the relaxation rates. Moreover, we also show physically meaningful examples that illustrate the physical nature of $\phi(k)$. In Sec. 3.3, we will show the numerical scheme, which calculates the relaxation rates from the linearized Boltzmann equation. The section that follows, Sec. 3.4, displays the results of the numerical scheme. We will show how the relaxation rates depend on the inverse final temperature, the chemical potential, and nextto-nearest-neighbor hopping parameter.

In the previous chapter, it was already been noted that the third relaxation rate is most important, because it is the thermalization rate. In Sec. 3.5, we will find an approximation for its eigenfunction. Then, we use this approximation to link this thermalization rate to an operator in state space. Afterwards, we will give an example on how approximating the eigenfunction helps us to determine the long-time temperature dependence for certain operators. As examples, the total particle current and the total energy current are used in Sec. 3.5.3.

In Sec. 3.6, we will calculate the first-order perturbation theory of the linearized Boltzmann equation in the next-to-nearest-neighbor hopping amplitude. Afterwards, it will be shown that the first order contribution of the lowest eigenvalues vanishes. We will show that this agrees with our numerical findings. 


\subsection{The Hubbard model and next-to-nearest-neighbor hopping}

The Hubbard model is a minimal model describing the valence electrons in a simple metal with one sort of atoms. It was invented by Hubbard (1963, 1964a,b); Gutzwiller (1963); Kanamori (1963). For one dimension, the Hubbard Hamiltonian (see also Eq. (1.69)) in the Wannier form is

$$
\hat{H}=-\sum_{l, m \in \mathbb{Z}} \sum_{\sigma \in\{\uparrow, \downarrow\}} J_{l m} \hat{c}_{l, \sigma}^{\dagger} \hat{c}_{m, \sigma}+\text { H.c. }+U \sum_{l \in \mathbb{Z}} \hat{n}_{l, \uparrow} \hat{n}_{l, \downarrow} .
$$

The first term is called the hopping term because it is the kinetic part of the Hamiltonian. The second term is the interaction term. It assumes that the Coulomb interaction is screened and consequently short-ranged. Therefore, in good approximation, electrons only interact, when they are at the same atom.

The most simple form of a hopping term assumes that the highest occupied atomic orbitals overlap only for neighboring atoms. This kind of hopping is called nearest-neighbor hopping and leads to the Hamiltonian

$$
\hat{H}=-J \sum_{l \in \mathbb{Z}} \sum_{\sigma \in\{\uparrow, \downarrow\}} \hat{c}_{l, \sigma}^{\dagger} \hat{c}_{l+1, \sigma}+H . c .+U \sum_{l \in \mathbb{Z}} \hat{n}_{l, \uparrow} \hat{n}_{l, \downarrow} .
$$

We denote this model as the integrable Hubbard model. It is integrable, because it has an infinite amount of conserved quantities in the thermodynamic limit. For this model, integrability can be broken by an additional hopping or interaction term. Thus, the integrable model is only a singular part of all possible Hubbard models and extended Hubbard models. It is important to note that a Boltzmann equation, like the one we will consider, cannot detect integrability breaking by an interaction term, because an additional interaction term would only modify $\left|\Phi_{\vec{\nu}, \vec{k}}\right|^{2}$. Therefore, the resulting Boltzmann equation would have the same conserved quantities as the one without an additional interaction term. Moreover, it is also not clear if it can detect integrability breaking by a hopping term. However, we will investigate the relaxation rates in the Boltzmann-equation approach when we extend the integrable Hubbard model by an additional next-to-nearest-neighbor hopping term, which takes into account the non-zero overlap of next-to-nearest valence orbitals. The Hamiltonian of the corresponding Hubbard model reads

$$
\hat{H}=\sum_{l \in \mathbb{Z}} \sum_{\sigma \in\{\uparrow, \downarrow\}}\left[-J \hat{c}_{l, \sigma}^{\dagger} \hat{c}_{l+1, \sigma}-J^{\prime} \hat{c}_{l, \sigma}^{\dagger} \hat{c}_{l+2, \sigma}\right]+H . c .+U \sum_{l \in \mathbb{Z}} \hat{n}_{l, \uparrow} \hat{n}_{l, \downarrow} .
$$

For simplicity, we define dimensionless parameters $\varepsilon:=J^{\prime} / J$ and $\beta:=2 J / k_{B} T$, where $T$ is the final temperature, which is dependent on the energy of the initial state. Furthermore, we introduce the dimensionless chemical potential $\mu$, which is measured in units of $2 J$.

The Boltzmann equation resulting from Eq. (3.3) was previously investigated by Fürst et al. (2012, 2013b). They found that for $J^{\prime}=0$, the quasiparticle momentum distributions do not thermalize, but relax to non-thermal stationary quasiparticle momentum distributions. The timescales for the relaxation 
are relatively fast. Conversely, for $J^{\prime} \neq 0$, the initial quasiparticle momentum distributions thermalize within the Boltzmann-equation description. Moreover, the timescales for the thermalization at $J \neq 0$ are much longer than the relaxation times of the integrable case. We will come back to this in Sec. 3.2.1 and Chapter 5 .

The Hubbard Hamiltonian has one single-particle band, therefore $N_{\mathrm{b}}=1$. In the Bloch form, cf. Eq. (2.2), it reads

$$
\begin{aligned}
\hat{H} & =\hat{H}_{0}+\hat{H}_{\text {int }} \\
\hat{H}_{0} & =2 J \int d k \sum_{\sigma} \omega(k) \hat{n}_{\sigma}(k) \\
\hat{H}_{\text {int }} & =U \int d^{4} k \delta\left(\operatorname{kmod}\left(\Delta k_{\vec{k}}\right)\right) \hat{c}_{\uparrow}^{\dagger}\left(k_{1}\right) \hat{c}_{\downarrow}^{\dagger}\left(k_{2}\right) \hat{c}_{\downarrow}\left(k_{3}\right) \hat{c}_{\uparrow}\left(k_{4}\right) .
\end{aligned}
$$

The comparison with Eq. (2.2) yields

$$
\Omega_{1}(k)=\frac{2 J}{\hbar} \omega(k) \quad \text { and } \quad \Phi_{\vec{k}}=1
$$

Moreover, the dispersion relation $\omega(k)$ of Eq. (3.4) is easily calculated from Eq. (3.3). It is

$$
\omega(k)=-\cos (2 \pi k)-\varepsilon \cos (4 \pi k) .
$$

The transformed creation operators are

$$
\hat{c}_{\sigma}(k)=\sum_{l} e^{-i 2 \pi k j} \hat{c}_{l, \sigma} .
$$

For the derivation of Eq. (3.4), see App. C.1.

\subsection{The Boltzmann equation for the Hubbard model}

In Sec. 3.1, we found the 1-band form of the Hubbard Hamiltonian. Using Eq. (3.5), the Boltzmann equation in Eq. (2.16) reduces to

$$
\dot{n}(k, t)=\mathcal{I}_{\text {coll }}[n](k, t),
$$

with the collision integral

$$
\begin{aligned}
\mathcal{I}_{\text {coll }}[n]_{1}= & \frac{2 \pi U^{2}}{\hbar^{2}} \int d k_{2} d k_{3} d k_{4} \delta\left(\Delta k_{\vec{k}}\right) \delta\left(2 J \Delta \omega_{\vec{k}} / \hbar\right) \\
& \times\left[\left(1-n_{1}\right)\left(1-n_{2}\right) n_{3} n_{4}-n_{1} n_{2}\left(1-n_{3}\right)\left(1-n_{4}\right)\right] \\
= & \frac{\pi}{\hbar J} \int d k_{2} d k_{3} d k_{4} \delta\left(\Delta k_{\vec{k}}\right) \delta\left(\Delta \omega_{\vec{k}}\right) \\
& \times\left[\left(1-n_{1}\right)\left(1-n_{2}\right) n_{3} n_{4}-n_{1} n_{2}\left(1-n_{3}\right)\left(1-n_{4}\right)\right] .
\end{aligned}
$$

To shorten the notation, we introduce $X_{j}=X\left(k_{j}, t\right)$ for $X=n, \phi$ and $Y_{j}=$ $Y\left(k_{j}, t\right)$ for $Y=f, \omega$. The two terms in the square brackets are the gain and the loss term taking into account Fermi-Dirac statistics. Furthermore, $\Delta k_{\vec{k}}=$ $\operatorname{kmod}\left(k_{1}+k_{2}-k_{3}-k_{4}\right)$ is the change in total momentum and $\Delta \omega_{\vec{k}}=\omega\left(k_{1}\right)+$ $\omega\left(k_{2}\right)-\omega\left(k_{3}\right)-\omega\left(k_{4}\right)$ is the change in energy. Defined in Eq. (2.3), the kmod function maps its parameter into the first Brillouin zone $\left[-\frac{1}{2}, \frac{1}{2}\right)$. It is important to note that the missing factor 2 in the prefactor comes from the 2 in Eq. (3.5). 

next-to-nearest-neighbor hopping

\subsubsection{Linearized Boltzmann equation}

In this section, we will apply the findings of the general section on the linearized Boltzmann equation (Sec. 2.2) to the Hubbard model. The linearized quasiparticle momentum distribution is

$$
n(k, t)=\frac{1}{1+\exp [\beta(\omega(k)-\mu)-\phi(k, t)]} \approx f(k)+f(k)[1-f(k)] \phi(k, t) .
$$

It leads to the rate equation (Eq. (2.20))

$$
\dot{\phi}(k, t)=-\hat{\mathcal{L}}[\phi](k, t) .
$$

In this equation, the linear, positive semidefinite, and hermitian operator $\hat{\mathcal{L}}$ is

$$
\begin{aligned}
\hat{\mathcal{L}}[\phi]_{1}= & \frac{\pi U^{2}}{\hbar J} \int d k_{2} d k_{3} d k_{4} \sum_{m=-1}^{1} \delta\left(\Delta k_{\vec{k}}+m\right) \delta\left(\Delta \omega_{\vec{k}}\right) \\
& \times F_{1234}\left[\phi_{1}+\phi_{2}-\phi_{3}-\phi_{4}\right]
\end{aligned}
$$

see Eq. (2.21). Here, we defined the factor $F_{1234}:=\left(1-f_{2}\right) f_{3} f_{4} / f_{1}$ containing all the Fermi-Dirac functions involved. It can be rewritten into

$$
\begin{aligned}
F_{1234} & =\left[\left(1-f_{1}\right)+f_{1}\right] \frac{\left(1-f_{2}\right) f_{3} f_{4}}{f_{1}} \\
& =\left(1-f_{1}\right) \frac{\left(1-f_{2}\right) f_{3} f_{4}}{f_{1}}+f_{1} \frac{\left(1-f_{2}\right) f_{3} f_{4}}{f_{1}} \\
& =\left(1-f_{1}\right) \frac{f_{2}\left(1-f_{3}\right)\left(1-f_{4}\right)}{1-f_{1}}+f_{1} \frac{\left(1-f_{2}\right) f_{3} f_{4}}{f_{1}} \\
& =f_{2}\left(1-f_{3}\right)\left(1-f_{4}\right)+\left(1-f_{2}\right) f_{3} f_{4} .
\end{aligned}
$$

Scalar product, norm and decomposition: For the single-band model, the scalar product defined in Eq. (2.22) looses the sum and becomes

$$
\langle\psi, \phi\rangle_{\mathrm{F}}:=\int d k \psi(k) f(k)[1-f(k)] \phi(k) .
$$

It induces the norm

$$
\|\psi\|_{\mathrm{F}}:=\sqrt{\langle\psi, \psi\rangle_{\mathrm{F}}}
$$

The operator $\hat{\mathcal{L}}$ is hermitian in this scalar product, see Eq. (B.64). The expansion in Eq. (2.27) is formally the same in the case of a single band:

$$
\phi(k, t)=\sum_{j} A_{j}(0) e^{-\lambda_{j} t} \chi^{(j)}(k) .
$$

Hereby, the coefficients $A_{j}(0)$ are determined by the initial state. The exponential factor shows that $\lambda_{j}$ are the relaxation rates we are looking for.

An eigenvalue $\lambda_{j}$, which is zero, means that the contribution $A_{j}(t)$ of the corresponding eigenfunction $\chi_{j}(k)$ persists over time. The other contributions decay exponentially in time. 
Fixed points: In this paragraph, we will identify fixed points of the Boltzmann equation established in Eq. (3.9). One fixed point, which is present for any dispersion relation, is the thermal distribution. In our case, it is the Fermi-Dirac distribution

$$
f(k)=\frac{1}{1+\exp [\beta(\omega(k)-\mu)]} .
$$

This means that the collision term vanishes for $n=f$, so that

$$
\mathcal{I}_{\text {coll }}[f] \equiv 0 \quad \forall \omega .
$$

The derivation can be found in App. C.7.

For the special case of the integrable Hubbard model, $\varepsilon=0$, there is a whole class of additional fixed points. Fürst et al. (2012) found stationary quasiparticle momentum distributions of the form

$$
f^{S}(k)=\frac{1}{1+\exp \left[\phi^{S}(k)-a\right]},
$$

where the 1-periodic function $\phi^{S}(k)$ is antisymmetric around $k= \pm \frac{1}{4}$ :

$$
\phi^{S}\left( \pm \frac{1}{4}+k\right)=-\phi^{S}\left( \pm \frac{1}{4}-k\right) .
$$

This means that the collision term vanishes for the stationary quasiparticle momentum distributions, cf. App. C.8:

$$
\left.\mathcal{I}_{\text {coll }}\left[f^{S}\right]\right|_{\varepsilon=0} \equiv 0
$$

The reason for the stationarity is the fact that $\left.\Delta \omega_{\vec{k}}\right|_{\varepsilon=0}=0$ is equivalent to $\operatorname{kmod}\left(k_{1}+k_{2}-\frac{1}{2}\right)=0$.

The relation between the perturbation $\phi(k, t)$ and the quasiparticle momentum distribution $n(k, t)$, as defined in Eq. (3.10), has the same form as the nonthermal stationary distributions $f^{S}(k)$, see Eq. (3.19). Thence, $\phi^{S}(k)$ is a fixed point of the linear operator for the integrable Hubbard model, i.e.

$$
\left.\hat{\mathcal{L}}\left[\phi^{S}\right]\right|_{\varepsilon=0} \equiv 0
$$

This means that every $\phi^{S}(k)$ fulfilling Eq. (3.20) is an eigenfunction of $\left.\hat{\mathcal{L}}\right|_{\varepsilon=0}$ and corresponds to the eigenvalue zero. The respective contribution to the total perturbation $\phi(k)$ is conserved.

\subsubsection{Separation and description of the non-trivial inter- action channel}

In Sec. 2.5, we introduced the interaction channels of the Boltzmann equation. The trivial channels we described in Sec. 2.5.1 do not contribute to $\mathcal{I}_{\text {coll }}$ and $\hat{\mathcal{L}}$. Only the non-trivial channels can contribute to $\mathcal{I}_{\text {coll }}$ and $\hat{\mathcal{L}}$. In Sec. 2.5.2, we proved that they exist, as long as the dispersion relation is continuous and 

next-to-nearest-neighbor hopping

periodic, like the dispersion relation $\omega(k)$ of the Hubbard model. In the following, we will demonstrate that the trivial channels can be separated from the non-trivial channels in the case of the Hubbard model.

Defined in Eq. (3.6), the dispersion relation reads

$$
\omega(k)=-\cos (2 \pi k)-\varepsilon \cos (4 \pi k) .
$$

To shorten our notation, we define

$$
\begin{array}{ll}
c_{k}:=\cos (2 \pi k), & \tilde{c}_{k}:=\cos (4 \pi k), \\
s_{k}:=\sin (2 \pi k), & \tilde{s}_{k}:=\sin (4 \pi k) .
\end{array}
$$

In order to simplify the calculation of the non-trivial interaction channels, we introduce new momentum coordinates

$$
\begin{aligned}
k:=\frac{1}{2}\left(k_{1}+k_{2}\right), & k^{\prime}:=\frac{1}{2}\left(k_{3}+k_{4}\right), \\
p:=\frac{1}{2}\left(k_{1}-k_{2}\right), & q:=\frac{1}{2}\left(k_{3}-k_{4}\right) .
\end{aligned}
$$

We will denote this set of momentum coordinates containing relative and total momenta as "center of mass and relative momentum coordinates". These variables are very well suited for describing scattering, since momentum conservation is trivially fulfilled by identifying $k=k^{\prime}$. The other set of coordinates, $\left\{k_{1}, k_{2}, k_{3}, k_{4}\right\}$, we call "original momentum coordinates", because they define the scattering process in the first place.

For writing the dispersion relation in the new variables, the first step is

$$
\begin{aligned}
& c_{k_{1}}+c_{k_{2}}=2 c_{k} c_{p} \\
& \tilde{c}_{k_{1}}+\tilde{c}_{k_{2}}=2 \tilde{c}_{k} \tilde{c}_{p}=2 \tilde{c}_{k}\left(2 c_{p}^{2}-1\right) .
\end{aligned}
$$

This holds similarly for $k_{1}, k_{2}, p \rightarrow k_{3}, k_{4}, q$. Thence, the energy conservation becomes

$$
\begin{aligned}
\Delta \omega_{\vec{k}} & =-c_{k_{1}}-c_{k_{2}}+c_{k_{3}}+c_{k_{4}}-\varepsilon\left[\tilde{c}_{k_{1}}+\tilde{c}_{k_{2}}-\tilde{c}_{k_{3}}-\tilde{c}_{k_{4}}\right] \\
& =-2 c_{k}\left(c_{p}-c_{q}\right)-2 \varepsilon \tilde{c}_{k}\left[\left(2 c_{p}^{2}-\not 1\right)-\left(2 c_{q}^{2}-\not 1\right)\right] \\
& =2\left(c_{p}-c_{q}\right)\left[c_{k}+2 \varepsilon \tilde{c}_{k}\left(c_{p}+c_{q}\right)\right] .
\end{aligned}
$$

If the factor $c_{p}-c_{q}$ is zero, we have trivial channels, see Eq. (2.47). In this case, we get an equation that describes the non-trivial channels only:

$$
c_{k}+2 \varepsilon \tilde{c}_{k}\left(c_{p}+c_{q}\right)=0
$$

One can easily consider two extreme cases: For $\varepsilon \rightarrow 0$, the second term of the l.h.s. in Eq. (3.28) vanishes and we are left with

$$
c_{k}=0 \text {. }
$$

This leads to

$$
k_{1}+k_{2}= \pm \frac{1}{2}
$$

Later on, we will see that increasing $\varepsilon$ a little bit will change the statement by $\mathcal{O}(\varepsilon)$. 
In the other extreme, $\varepsilon \rightarrow \infty$, there is no nearest-neighbor hopping, only nextto-nearest-neighbor hopping. Thus, the chain splits into two independent ones: The chain with the odd positions and the chain with the even positions. Thence, the band $-\varepsilon \cos (4 \pi k)$ with $k \in \mathbb{K}$ is split into two independent bands. The first band is $\omega_{1}\left(k^{\prime}\right)=-\cos \left(2 \pi k^{\prime}\right)$, with $k^{\prime}=2 k$ and $k \in\left(-\frac{1}{4}, \frac{1}{4}\right]$. The second band looks similar, $\omega_{2}\left(k^{\prime}\right)=-\cos \left(2 \pi k^{\prime}\right)$, but now $k^{\prime}=\operatorname{kmod}(2 k)$ and $k \in$ $\left(-\frac{1}{2},-\frac{1}{4}\right] \wedge\left(-\frac{1}{4}, \frac{1}{2}\right]$. Hence, we have two bands with $k^{\prime}=\operatorname{kmod}(2 k) \in \mathbb{K}$.

The trivial interaction channels come from $c_{p^{\prime}}-c_{q^{\prime}}=0$, as demonstrated in Eq. (2.47). With the primed variables being twice the unprimed variables, we obtain

$$
0=c_{p^{\prime}}-c_{q^{\prime}}=\tilde{c}_{p}-\tilde{c}_{q}=2\left(c_{p}-c_{q}\right)\left(c_{p}+c_{q}\right) .
$$

Let us compare this to the full energy conservation, Eq. (3.27). In our case, only the second term $4 \varepsilon \tilde{c}_{k}\left(c_{p}-c_{q}\right)\left(c_{p}+c_{q}\right)$ contributes. It describes the trivial channels correctly, because it contains the factor $\tilde{c}_{p}-\tilde{c}_{q}$. The remaining factor, $\tilde{c}_{k}=0$, is equivalent to $c_{k^{\prime}}=0$. This describes the non-trivial channels and is consistent with the finding for one chain, i.e. $\varepsilon=0$, see Eq. (3.29).

Now that we have established that the non-trivial channels are consistent and physically reasonable in the extreme cases, we consider the solutions between the two extreme cases, i.e. for general next-to-nearest-neighbor hopping $\varepsilon$. Therefore, we consider the non-trivial solutions

$$
c_{k}+2 \varepsilon r_{p q}\left(2 c_{k}^{2}-1\right)=0,
$$

where we defined $r_{p q}:=\left(c_{p}+c_{q}\right)$. The momenta $k$, which solve Eq. (3.32) are denoted by $\tilde{k}_{p q}^{\varepsilon}$. Completing the square, we find

$$
\cos \left(2 \pi \tilde{k}_{p q}^{\varepsilon}\right)=\frac{-1 \pm \sqrt{1+32\left(\varepsilon r_{p q}\right)^{2}}}{8 \varepsilon r_{p q}} .
$$

Next, we show that for $\varepsilon<\frac{1}{4}$ the solution with the minus sign is not valid. Setting $x:=4 \varepsilon r_{p q}$, the right-hand side is

$$
\left|\frac{-1-\sqrt{1+2 x^{2}}}{2 x}\right| \leqslant 1 \Longleftrightarrow \sqrt{1+2 x^{2}} \leqslant 2|x|-1 .
$$

If $|x|<1$, the inequality cannot be fulfilled, since $\sqrt{1+2 x^{2}} \geqslant 1$. In the other case, the right-hand side is bigger than zero. Hence, we are allowed to square both sides and get

$$
\Longleftrightarrow 2 x^{2} \leqslant 4 x^{2}-4|x| \Longleftrightarrow 0 \leqslant|x|(|x|-2) .
$$

For $|x|<2$, this cannot be fulfilled. Thus, for $\varepsilon=\frac{|x|}{4\left|r_{p q}\right|} \leqslant \frac{|x|}{8}<\frac{1}{4}$, the minus sign solution is not valid.

The limiting case is $\varepsilon=\frac{1}{4}$. Thence, we have

$$
\begin{aligned}
& x= \pm 2 \Leftrightarrow r_{p q}= \pm 2 \Leftrightarrow p=q=\frac{n}{2} \text { with } n \in \mathbb{Z} \\
\Leftrightarrow & k_{1}-k_{2}=2 k_{3}-k_{1}-k_{2}=n \text { with } n \in \mathbb{Z} \\
\Leftrightarrow & k_{1}=k_{3} \wedge k_{2}=k_{1}+n \text { with } n \in \mathbb{Z} .
\end{aligned}
$$



next-to-nearest-neighbor hopping

Shifting the $k_{j}$ into the Brillouin zone $\mathbb{K}=\left(-\frac{1}{2}, \frac{1}{2}\right]$, this equation describes the point $k_{1}=k_{2}=k_{3}=k_{4}$ as a special coincidence of the trivial channels. Hence, for $\varepsilon=\frac{1}{4}$, the additional non-trivial channel is actually coinciding with trivial channels. It is important to note that for $\varepsilon>\frac{1}{4}$, the additional channel does not coincide with trivial channels in general.

We have proven that Eq. (3.33) does not have the solution with the minus sign when

$$
\varepsilon \leqslant \frac{1}{4}
$$

This is sufficient to describe small integrability breaking. Thus, from now on, we will only consider this case. This is also motivated by the fact that for $\varepsilon>\frac{1}{4}$ we would have to implement the additional interaction channel into our numerical code.

The plus-sign solution of Eq. (3.33) is valid for all $\varepsilon r_{p q}$ :

$$
\left|\frac{-1+\sqrt{1+2 x^{2}}}{2 x}\right| \leqslant 1 \Longleftrightarrow \sqrt{1+2 x^{2}} \leqslant 2|x|+1 \Longleftrightarrow 0 \leqslant 2 x^{2}+4|x| .
$$

Thus, in our case, we obtain

$$
\cos \left(2 \pi \tilde{k}_{p q}^{\varepsilon}\right)=\frac{\sqrt{1+32\left(\varepsilon r_{p q}\right)^{2}}-1}{8 \varepsilon r_{p q}} .
$$

For $\varepsilon=0$, the non-trivial interaction channel yields $\tilde{k}_{p q}^{0}= \pm \frac{1}{4}$, see Eq. (3.30). Therefore, we define

$$
d_{p q}^{\varepsilon}:=\tilde{k}_{p q}^{\varepsilon}-\frac{1}{4}
$$

This yields

$$
\sin \left(2 \pi d_{p q}^{\varepsilon}\right)=\frac{\sqrt{1+32\left(\varepsilon r_{p q}\right)^{2}}-1}{8 \varepsilon r_{p q}} .
$$

This equation has two solutions and we choose

$$
d_{p q}^{\varepsilon}=\frac{1}{2 \pi} \arcsin \left\{\frac{\sqrt{1+32\left(\varepsilon r_{p q}\right)^{2}}-1}{8 \varepsilon r_{p q}}\right\} \in\left[-\frac{1}{4}, \frac{1}{4}\right] .
$$

Hence, we obtain $\tilde{k}_{p q}^{\varepsilon} \in\left[0, \frac{1}{2}\right]$ and

$$
\tilde{k}_{p q}^{\varepsilon}:=+\frac{1}{2 \pi} \arccos \left\{\frac{\sqrt{1+32\left(\varepsilon r_{p q}\right)^{2}}-1}{8 \varepsilon r_{p q}}\right\} .
$$

However, we must keep in mind that $-\tilde{k}_{p q}^{\varepsilon}$ is also a solution of the equation $\Delta \omega_{\vec{k}}=0$. At this point, we notice that $\tilde{k}_{p q}^{\varepsilon}$ and $d_{p q}^{\varepsilon}$ only depend on $\varepsilon r_{p q}$. Furthermore, $d_{p q}^{\varepsilon}$ is an odd function of $\varepsilon r_{p q}$. A Taylor expansion around $\varepsilon=0$ yields

$$
d_{p q}^{\varepsilon}=-\frac{1}{\pi} \varepsilon r_{p q}+\frac{22}{3 \pi}\left(\varepsilon r_{p q}\right)^{3}+O\left(\varepsilon r_{p q}\right)^{5} .
$$


Therefore, we get

$$
\tilde{k}_{p q}^{\varepsilon}=\frac{1}{4}+\mathcal{O}(\varepsilon) \text {. }
$$

This leads to the fact that non-trivial interaction channels are altered by $\mathcal{O}(\varepsilon)$ when turning on the next-to-nearest-neighbor hopping.

The solution $\tilde{k}_{p q}^{\varepsilon}$ can be expressed using the original momentum coordinates. However, it can not be solved for one of the $k_{j}$. But the Taylor expansion can be found using an iterative approach. As an example, we demonstrate the iteration for $k_{2}$. It starts using the zeroth order expansion

$$
k_{2}= \pm 2 \tilde{k}_{p q}^{\varepsilon}-k_{1}= \pm \frac{1}{2}+\mathcal{O}(\varepsilon) .
$$

This is plugged into the r.h.s. of Eq. (3.43), which yields the first-order expansion

$$
\begin{aligned}
k_{1}+k_{2} & = \pm \frac{1}{2}-\varepsilon \Delta_{13}, \\
\Delta_{13} & =\frac{2}{\pi}\left(s_{k_{1}}+s_{k_{3}}\right)+O\left(\varepsilon^{3}\right) .
\end{aligned}
$$

Hence, the $n^{\text {th }}$ iteration step is plugging the $n^{\text {th }}$-order expansion into the r.h.s. of Eq. (3.43), which leads to the $(n+1)^{\text {st }}$-order expansion. This way, the Taylor expansion can be calculated up to every finite order. The third-order expansion of $k_{1}+k_{2}= \pm \frac{1}{2}-\varepsilon \Delta_{13}$ yields

$$
\Delta_{13}=\frac{2}{\pi}\left(s_{k_{1}}+s_{k_{3}}\right)\left[1+2 \varepsilon\left(c_{k_{1}}+c_{k_{3}}\right)+4 \varepsilon^{2}\left[\left(c_{k_{1}}+c_{k_{3}}\right)^{2}-\frac{7}{3}\left(s_{k_{1}}+s_{k_{3}}\right)^{2}\right]\right]+\mathcal{O}\left(\varepsilon^{3}\right) .
$$

The fact that $\tilde{k}_{p q}^{\varepsilon}=\frac{1}{4}+\mathcal{O}(\varepsilon)$ leads to very tight bounds on $\tilde{k}_{p q}^{\varepsilon}$. Rough bounds can be found easily by noticing that $\tilde{k}_{p q}^{\varepsilon}$ is a monotonic function of $\varepsilon r_{p q}$ :

$$
\begin{aligned}
& \frac{d}{d x} \frac{\sqrt{1+2 x^{2}}-1}{2 x}=\frac{1}{\sqrt{1+2 x^{2}}}-\frac{\sqrt{1+2 x^{2}}-1}{2 x^{2}} \\
& =\frac{1}{2 x^{2}}+\frac{1}{\sqrt{1+2 x^{2}}}\left[1-\frac{1+2 x^{2}}{2 x^{2}}\right]=\frac{1}{2 x^{2}}\left[1-\frac{1}{\sqrt{1+2 x^{2}}}\right] \\
& =\frac{1}{2 x^{2} \sqrt{1+2 x^{2}}}\left[\sqrt{1+2 x^{2}}-1\right] \geqslant 0 \quad \forall x .
\end{aligned}
$$

Furthermore, we make use of the fact that the cosine is bounded, in particular $-2 \leqslant r_{p q} \equiv c_{p}+c_{q} \leqslant 2$. Due to the monotonicity of $\tilde{k}_{p q}^{\varepsilon}$, we obtain bounds of $\tilde{k}_{p q}^{\varepsilon}$ by plugging the bounds on $r_{p q}$ into Eq. (3.43):

$$
-\frac{\sqrt{1+128 \varepsilon^{2}}-1}{16 \varepsilon} \leqslant \cos \left(2 \pi \tilde{k}_{p q}^{\varepsilon}\right) \leqslant \frac{\sqrt{1+128 \varepsilon^{2}}-1}{16 \varepsilon} .
$$

We defined $\tilde{k}_{p q}^{\varepsilon}$ to be non-negative. With the cosine being monotonically decreasing in the parameter range $\left(0, \frac{1}{2}\right)$, we obtain

$$
\frac{1}{2 \pi} \arccos \left(\frac{\sqrt{1+128 \varepsilon^{2}}-1}{16 \varepsilon}\right) \leqslant \tilde{k}_{p q}^{\varepsilon} \leqslant \frac{1}{2 \pi} \arccos \left(-\frac{\sqrt{1+128 \varepsilon^{2}}-1}{16 \varepsilon}\right) .
$$




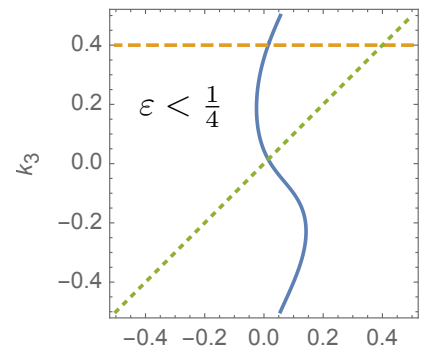

(A)

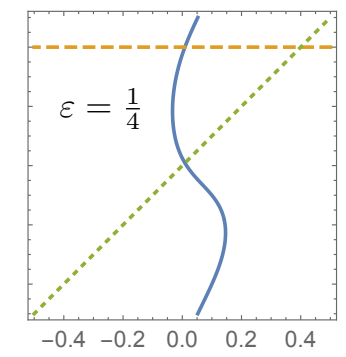

(B) next-to-nearest-neighbor hopping

Figure 3.1: Emergence of the additional non-trivial interaction channel. In all pictures, we set $k_{1}=0.4$. The next-to-nearest-neighbor hopping parameter is $\varepsilon=0.22$ (A), $\varepsilon=0.25(\mathrm{~B})$, and $\varepsilon=0.27(\mathrm{C})$. In the last picture, an additional solution appears in form of a circle. It is the minus-sign solution of Eq. (3.58).

For very small $\varepsilon$ :

$$
\frac{1}{4}-\frac{2 \varepsilon}{\pi}+\mathcal{O}\left(\varepsilon^{3}\right)<\tilde{k}_{p q}^{\varepsilon}<\frac{1}{4}+\frac{2 \varepsilon}{\pi}+\mathcal{O}\left(\varepsilon^{3}\right) .
$$

Thus, the solution $\tilde{k}_{p q}^{\varepsilon}$ is bound to a small region around $\frac{1}{4}$. The region is of $\mathcal{O}(\varepsilon)$. Even for our maximal next-to-nearest-neighbor hopping, $\varepsilon=\frac{1}{4}$, the region is still quite narrow:

$$
\frac{1}{6} \leqslant \tilde{k}_{p q}^{\varepsilon} \leqslant \frac{1}{3}
$$

We conclude that for weakly broken integrability ( $($ mall $\varepsilon$ ), the interaction channels change only slightly. However, it is not clear yet if this is sufficient to see thermalization within the Boltzmann equation's description. We will address this issue in Sec. 3.4.

\subsubsection{Uniqueness of the non-trivial interaction channel for the Hubbard model}

In this section, we make a contribution to the implementation of the numerical scheme that diagonalizes the linear Boltzmann operator $\hat{\mathcal{L}}$. We investigate the energy conservation $\Delta \omega_{\vec{k}}=0$, written in the original momentum coordinates $k_{1}, k_{2}, k_{3}$, and $k_{4}$. The momentum $k_{4}$ is fixed by momentum conservation: $k_{4}=k_{1}+k_{2}-k_{3}$. Since $\hat{\mathcal{L}}[\phi]\left(k_{1}\right)$ depends on $k_{1}$, the momentum $k_{1}$ is also fixed. The two integrations over $k_{2}$ and $k_{3}$ are left. In general, one has to scan all three parameters $\left(k_{2}, k_{3}\right) \in \mathbb{K}^{3} \equiv\left(-\frac{1}{2}, \frac{1}{2}\right]^{3}$ and find the zeros of the function $\left(k_{1}, k_{2}, k_{3}\right) \mapsto \Delta \omega_{\vec{k}}$. However, for the Hubbard model with next-tonearest-neighbor hopping, there is a big simplification making the numerical implementation considerably simpler: Given $k_{1}$ and $k_{3}$, there is exactly one non-trivial $k_{2}$, which fulfills the energy conservation. In the following, we will proof this statement.

Let us rearrange the energy conservation $\Delta \omega_{\vec{k}}=0$ :

$$
\omega\left(k_{2}\right)-\omega\left(k_{2}+k_{1}-k_{3}\right)=\omega\left(k_{3}\right)-\omega\left(k_{1}\right) .
$$


We can rewrite the left side in the form

$$
f(x):=\omega(x+z)-\omega(x-z),
$$

with the variable $x=k_{2}+\frac{1}{2}\left(k_{1}-k_{3}\right)$ and the constant $z=\frac{1}{2}\left(k_{3}-k_{1}\right)$. Then, we define $C:=\omega\left(k_{3}\right)-\omega\left(k_{1}\right)$. Thus, Eq. (3.54) becomes

$$
f(x)=C .
$$

This equation can have two solutions for $x$ at most, because the function $f(x)$ has exactly one maximum and one minimum in the interval $\left(-\frac{1}{2}, \frac{1}{2}\right]$ for $\varepsilon \leqslant \frac{1}{4}$. The extrema are the solutions of

$$
\begin{aligned}
0 & =\frac{d}{d x}[\omega(x+z)-\omega(x-z)] \\
& =2 \pi\left[s_{x+z}-s_{x-z}\right]+4 \pi \varepsilon\left[\tilde{s}_{x+z}-\tilde{s}_{x-z}\right] \\
& =4 \pi s_{z}\left[c_{x}+4 \varepsilon \tilde{c}_{x} c_{z}\right] \\
& =4 \pi s_{z}\left[c_{x}+8 \varepsilon c_{x}^{2} c_{z}-4 \varepsilon c_{z}\right] .
\end{aligned}
$$

This is solved by

$$
\Rightarrow c_{x}=\frac{-1 \pm \sqrt{1+2 \alpha^{2}}}{2 \alpha},
$$

with $\alpha:=8 \varepsilon c_{z}$. The solution with the positive sign gives two solutions (if $x=x_{0}$ is a solution, so is $x=-x_{0}$, because of $c_{-x}=c_{x}$ ).

Considering Eq. (3.58) with the negative sign, there is no solution as long as $\varepsilon<\frac{1}{4}$, because $-1 \leqslant c_{x} \leqslant 1$. This can be proven by considering the minimum value of $c_{x}$, which is -1 :

$$
\begin{aligned}
& -1=\frac{-1-\sqrt{1+2 \alpha^{2}}}{2 \alpha} \\
& \Leftrightarrow 2 \alpha=1+\sqrt{1+2 \alpha^{2}} .
\end{aligned}
$$

The l.h.s. shows that $\alpha$ has to be bigger than 1 . Thus, we have

$$
\begin{aligned}
& 4 \alpha^{2}-4 \alpha+1=1+2 \alpha^{2} \\
& \Leftrightarrow 0=2 \alpha^{2}-4 \alpha=2 \alpha(\alpha-2) .
\end{aligned}
$$

$\alpha=0$ is not a solution, because $\alpha>1$. Therefore, we obtain

$$
\alpha=2 \Leftrightarrow c_{z}=\frac{1}{4 \varepsilon} .
$$

This can only work for $\varepsilon \geqslant \frac{1}{4}$.

So far, we have shown that the function $\omega(x+z)-\omega(x-z)$ has exactly two extrema in $x \in\left(-\frac{1}{2}, \frac{1}{2}\right]$. These extrema can only be one minimum and one maximum because of the continuity and the periodicity of the dispersion relation $\omega(k)$. Between two consecutive extrema, the function has to be monotonic. Thus, setting the function equal to a constant, there can only be one solution between two consecutive extrema. Therefore, there are exactly two solutions of $\omega(x+z)-\omega(x-z)=C$, as long as the constant $C$ does not lie on the extreme 

next-to-nearest-neighbor hopping

values or exceeds them. The constant $C$ cannot exceed the function values, because it is a value of the function $f(x)$ :

$$
C=f\left(\frac{k_{3}+k_{1}}{2}\right) .
$$

Approaching an extremum, the two solutions lie closer together. At the extremum, the trivial solution $k_{2}=k_{3}$ and the non-trivial solution coincide. Thus, there is one point where the non-trivial solution is actually trivial. For every $k_{1}$, there is a $k_{3}$-value, where the trivial and the non-trivial solution coincide.

For the case of $k_{1}=k_{3}$, obviously all $k_{2}$ are possible solutions, but these are of course trivial solutions. In this case, there is one $k_{2}$, where the solution coincides with the non-trivial channel.

To visualize this, we consider the space $\left(k_{2}, k_{3}\right) \in \mathbb{K}^{2}$ with a fixed $k_{1}$ in Fig. 3.1. There, we plot all the points having a solution to the energy conservation. This gives us three continuous lines: The non-trivial solution's line, and the two trivial solutions' straight lines $k_{2}=k_{1}$ and $k_{2}=k_{3}$. Indeed one can see that, at one point, the trivial solution $k_{2}=k_{3}$ and the non-trivial solution coincide. Moreover, in the case of $k_{1}=k_{3}$, one sees that there is also one point, where the solutions coincide, i.e. the previously mentioned continuation.

Fig. 3.1 also shows that for $\varepsilon>\frac{1}{4}$, there is an additional line forming a circle. It stems from the solution with the minus sign in Eq. (3.58). For $\varepsilon=\frac{1}{4}$, these solutions are contracted to a single point. Hence, in principle, this limiting case also has additional extrema in the function $f(x)$ :

$$
\begin{aligned}
& \pm 1=\frac{-1-\sqrt{1+8 c_{z}^{2}}}{4 c_{z}} \wedge c_{x}= \pm 1 \\
\Leftrightarrow & \mp 4 c_{z}-1=\sqrt{1+8 c_{z}^{2}} \wedge c_{x}= \pm 1 \\
\Leftrightarrow & 16 c_{z}^{2} \pm 8 c_{z}+1=1+8 c_{z}^{2} \wedge \mp 4 c_{z}-1>1 \wedge c_{x}= \pm 1 \\
\Leftrightarrow & 8 c_{z}^{2} \pm 8 c_{z}=0 \wedge \mp c_{z}>\frac{1}{2} \wedge c_{x}= \pm 1 \\
\Leftrightarrow & \left(c_{z}=\mp 1 \vee c_{z}=0\right) \wedge \mp c_{z}>\frac{1}{2} \wedge c_{x}= \pm 1 \\
\Leftrightarrow & c_{z}=\mp 1 \wedge \mp c_{z}>\frac{1}{2} \wedge c_{x}= \pm 1 \\
\Leftrightarrow & c_{z}=\mp 1 \wedge c_{x}= \pm 1 \\
\Leftrightarrow & \bigvee\left[\left(z=n \wedge x=\frac{1}{2}+m\right) \vee\left(z=\frac{1}{2}+n \wedge x=m\right)\right] . \\
& m, n \in \mathbb{Z}
\end{aligned}
$$

Using $x=k_{2}+\frac{1}{2}\left(k_{1}-k_{3}\right)$ and $z=\frac{1}{2}\left(k_{3}-k_{1}\right)$, we obtain

$$
\begin{aligned}
& \bigvee\left[\left(z=n \wedge x=\frac{1}{2}+m\right) \vee\left(z=\frac{1}{2}+n \wedge x=m\right)\right] \\
& \Leftrightarrow \bigvee_{m, n \in \mathbb{Z}}\left[\left(\frac{1}{2}\left(k_{3}+k_{1}\right)=n \wedge k_{2}+\frac{1}{2}\left(k_{1}-k_{3}\right)=\frac{1}{2}-m\right)\right. \\
& \left.\quad \vee\left(\frac{1}{2}\left(k_{3}-k_{1}\right)=\frac{1}{2}+n \wedge k_{2}+\frac{1}{2}\left(k_{1}-k_{3}\right)=m\right)\right] \\
\Leftrightarrow & \bigvee_{m, n \in \mathbb{Z}}\left[\left(k_{3}=k_{1}+2 n \wedge k_{2}=\frac{1}{2}+m-n\right) \vee\left(k_{3}=k_{1}+1+2 n \wedge k_{2}=\frac{1}{2}+n+m\right)\right] .
\end{aligned}
$$


Shifting the momenta $k_{j}$ into the Brillouin zone $\mathbb{K}=\left(-\frac{1}{2}, \frac{1}{2}\right]$, we are left with the single solution

$$
k_{3}=k_{1} \wedge k_{2}=\frac{1}{2} .
$$

It follows that the two additional extrema coincide and we thus only have one additional extremum. Having three extrema in the Brillouin zone means that one still has one maximum and one minimum. The third extremum is either the same as the minimum or the maximum or it is an inflection point. Either way, the reasoning for the unique solution for $\varepsilon<\frac{1}{4}$ is still valid here for $\varepsilon=\frac{1}{4}$. It is important to note that this is consistent with the fact that the additional non-trivial channel coincides with a trivial channel for $\varepsilon=\frac{1}{4}$, see Eq. (3.36).

In summary, for $\varepsilon \leqslant \frac{1}{4}$ there is a unique non-trivial solution

$$
k_{2}=\tilde{k}_{2}\left(k_{1}, k_{3}\right)
$$

solving the energy conservation for a fixed $k_{1}$ and $k_{3}\left(k_{4}\right.$ follows from momentum conservation). Hence, the algorithm for the numerical scheme is as follows:

1. Fix the momenta $k_{1}$ and $k_{3}$, for which a solution is supposed to be found.

2. Calculate an approximate solution using the Taylor expansion of $\tilde{k}_{2}\left(k_{1}, k_{3}\right)=$ $\frac{1}{2}-k_{1}-\Delta_{13}$, see Eq. (3.48).

3. Use a root-finding algorithm like the Newton-Raphson procedure to find the roots of the non-trivial part of the energy conservation, see Eq. (3.28).

\subsubsection{The reformulation of the delta distribution of the energy conservation}

For both analytical and numerical calculation, it is inevitable to rewrite the delta-distribution $\delta\left(\Delta \omega_{\vec{k}}\right)$, which is a factor of the collision term $\mathcal{I}_{\text {coll }}$ and the linearized Boltzmann operator. The first paragraph treats $\delta\left(\Delta \omega_{\vec{k}}\right)$ written with the original momentum coordinates $k_{1}, k_{2}, k_{3}$, and $k_{4}$, which we use for the numerical evaluation, the second paragraph deals with $\delta\left(\Delta \omega_{\vec{k}}\right)$ using the center of mass and relative momentum coordinates $k, p$, and $q$, which is necessary for the analytical calculations.

Original momentum coordinates: Using the momentum conservation $k_{4}=$ $k_{1}+k_{2}-k_{3}$, we can express $\delta\left(\Delta \omega_{\vec{k}}\right)$ with the original momentum coordinates, $k_{1}, k_{2}, k_{3}$. Fixing the tupel $\left(k_{1}, k_{3}\right)$, we obtain a unique non-trivial solution $k_{2}=\tilde{k}_{2}\left(k_{1}, k_{3}\right)$, see Sec. 3.2.3. The delta-distribution becomes

$$
\delta\left(\Delta \omega_{\vec{k}}\right)=\frac{\delta\left(k_{2}-\tilde{k}_{2}\left(k_{1}, k_{3}\right)\right)}{\left|\frac{d \omega}{d k}\left(\tilde{k}_{2}\left(k_{1}, k_{3}\right)\right)-\frac{d \omega}{d k}\left(\tilde{k}_{4}\left(k_{1}, k_{3}\right)\right)\right|},
$$

with the group velocity $\frac{d \omega}{d k}=2 \pi[\sin (2 \pi k)+2 \varepsilon \sin (4 \pi k)]$. This removes the integration over $k_{2}$. Since the $k_{4}$ integration is removed by the momentumconservation, the only integration left is the one over $k_{3}$. 

next-to-nearest-neighbor hopping

Center of mass and relative momentum coordinates: Now, we simplify $\delta\left(\Delta \omega_{k, p, q}^{\varepsilon}\right)$ with $\Delta \omega_{k, p, q}^{\varepsilon}=\omega(k+p)+\omega(k-p)-\omega(k+q)-\omega(k-q)$. From Sec. 3.2.2, we obtain the solutions of $\Delta \omega_{k, p, q}^{\varepsilon}=0$. They are $\tilde{k}_{p q}^{\varepsilon}$ and $-\tilde{k}_{p q}^{\varepsilon}$. The energy-conserving delta function becomes

$$
\begin{aligned}
\delta\left(\Delta \omega_{k, p, q}^{\varepsilon}\right) & =\sum_{\substack{k_{0}(p, q) \in\left[-\frac{1}{2}, \frac{1}{2}\right] \\
\Delta \omega_{\vec{k}}\left(k_{0}(p, q), p, q\right)=0}} \delta\left(k-k_{0}(p, q)\right)\left|\left[\frac{\partial \Delta \omega_{k, p, q}^{\varepsilon}}{\partial k}\right]_{k=k_{0}(p, q)}\right|^{-1} \\
& =\delta\left(k-\tilde{k}_{p q}^{\varepsilon}\right)\left|\left[\frac{\partial \Delta \omega_{k, p, q}^{\varepsilon}}{\partial k}\right]_{k=\tilde{k}_{p q}^{\varepsilon}}\right|^{-1}+\delta\left(k+\tilde{k}_{p q}^{\varepsilon}\right)\left|\left[\frac{\partial \Delta \omega_{k, p, q}^{\varepsilon}}{\partial k}\right]_{k=-\tilde{k}_{p q}^{\varepsilon}}\right|^{-1} .
\end{aligned}
$$

To calculate the derivative of $\Delta \omega_{\vec{k}}$, we use $\Delta \omega_{\vec{k}}=2\left(c_{p}-c_{q}\right)\left[c_{k}+2 \varepsilon \tilde{c}_{k}\left(c_{p}+c_{q}\right)\right]$, see Eq. (3.27). This leads to

$$
\begin{aligned}
\left|\frac{\partial \Delta \omega_{k, p, q}^{\varepsilon}}{\partial k}\right| & =\left|\frac{\partial}{\partial k} 2\left(c_{p}-c_{q}\right)\left[c_{k}+2 \varepsilon r_{p q} \tilde{c}_{k}\right]\right| \\
& =4 \pi\left|\left(c_{p}-c_{q}\right)\left[s_{k}+4 \varepsilon r_{p q} \tilde{s}_{k}\right]\right| \\
& =4 \pi\left|s_{k}\right|\left|c_{p}-c_{q}\right|\left[1+8 \varepsilon r_{p q} c_{k}\right] .
\end{aligned}
$$

In the last factor of the last line, we may omit the absolute value, because

$$
1+8 \varepsilon r_{p q} c_{\tilde{k}_{p q}^{\varepsilon}}=1+8 \varepsilon r_{p q} \frac{\sqrt{1+32\left(\varepsilon r_{p q}\right)^{2}}-1}{8 \varepsilon r_{p q}}=\sqrt{1+32\left(\varepsilon r_{p q}\right)^{2}} \geqslant 1 .
$$

Moreover, $\left|\partial \Delta \omega_{\vec{k}} / \partial k\right|$ is symmetric in $k$. Next, we plug in the solution $\tilde{k}_{p q}^{\varepsilon}$ :

$$
\left|\left[\frac{\partial \Delta \omega_{k, p, q}^{\varepsilon}}{\partial k}\right]_{k= \pm \tilde{k}_{p q}^{\varepsilon}}\right|=4 \pi s_{\tilde{k}_{p q}^{\varepsilon}}\left|c_{p}-c_{q}\right| \sqrt{1+32\left(\varepsilon r_{p q}\right)^{2}} .
$$

Here, we dropped the absolute value in $\left|s_{\tilde{k}_{p q}^{\varepsilon}}\right|$, because $s_{\tilde{k}_{p q}^{\varepsilon}}$ is non-negative due to the fact that we defined $\tilde{k}_{p q}^{\varepsilon} \in\left[0, \frac{1}{2}\right]$, see Eq. (3.42). Furthermore, we obtain

$$
\frac{1}{\left|d \Delta \omega_{\vec{k}} / d \tilde{k}_{p q}^{\varepsilon}\right|}=\frac{1}{4 \pi} \frac{1}{\left|c_{p}-c_{q}\right|} \underbrace{\frac{1}{s_{\tilde{k}_{p q}^{\varepsilon}}} \frac{1}{\sqrt{1+32\left(\varepsilon r_{p q}\right)^{2}}}}_{=: \gamma\left(\varepsilon r_{p q}\right)} .
$$

The function $\gamma(x)$ is even and positive. It does not diverge, because $\tilde{k}_{p q}^{\varepsilon}$ does neither reach 0 nor $\frac{1}{2}$, see Eq. (3.51). This function is

$$
\begin{aligned}
\gamma(x) & =\frac{1}{\sqrt{1-c_{\tilde{k}_{p q}^{\varepsilon}}^{2}}} \frac{1}{\sqrt{1+32\left(\varepsilon r_{p q}\right)^{2}}} \\
& =\left[1-\left(\frac{\sqrt{1+32 x^{2}}-1}{8 x}\right)^{2}\right]^{-1 / 2} \frac{1}{\sqrt{1+32 x^{2}}} \\
& =\frac{1}{\sqrt{32 x^{2}+2\left(\sqrt{1+32 x^{2}}-1\right)}} \frac{1}{\sqrt{1+32 x^{2}}} \\
& =1-14 x^{2}+326 x^{4}+\mathcal{O}\left(x^{6}\right) .
\end{aligned}
$$


To summarize, we find

$$
\delta\left(\Delta \omega_{k, p, q}^{\varepsilon}\right)=\frac{1}{4 \pi} \frac{\gamma\left(\varepsilon r_{p q}\right)}{\left|c_{p}-c_{q}\right|}\left\{\delta\left(k-\tilde{k}_{p q}^{\varepsilon}\right)+\delta\left(k+\tilde{k}_{p q}^{\varepsilon}\right)\right\}
$$

The advantage of this form of $\delta\left(\Delta \omega_{\vec{k}}\right)$ is the separation of the diverging factor $1 /\left|c_{p}-c_{q}\right|$ from the $\varepsilon$-dependent factor. This is used for computing the first-order perturbation theory in Sec. 3.6.4.

\subsubsection{Examples for an initial perturbation}

At this point, we present two examples for initial perturbations $\phi_{0}(k)$. They should give an impression on the meaning of the perturbation $\phi(k, t)$. We will investigate an electric field and a temperature gradient, which create particle and heat currents in electronic systems. Each of them creates a certain initial quasiparticle momentum distributions. Our method investigates the relaxation of these two initial distributions when the electric field and the temperature gradient are turned off.

Example 1: Electric field: The first example is created by applying a homogeneous electric field $E$ to a Fermi-distribution for a time $\Delta t_{\mathrm{E}}$. The initial temperature is $T_{0}$, the initial chemical potential $\mu_{0}$. During the application of the field, we have

$$
\hbar \dot{k}(t)=e E .
$$

After the time $\Delta t_{\mathrm{E}}$, this results in a shift of the distribution,

$$
f(k) \rightarrow n_{0}(k)=f\left(k+\Delta k_{\mathrm{E}}\right)=\frac{1}{1+\exp \left[\beta_{0}\left(\omega\left(k+\Delta k_{\mathrm{E}}\right)-\mu_{0}\right)\right]},
$$

with $\Delta k_{\mathrm{E}}=\int_{0}^{\Delta t_{\mathrm{E}}} d t \dot{k}(t)=\Delta t_{\mathrm{E}} e E / \hbar$. We assume that the $\Delta k_{\mathrm{E}}$ is small. To read off the initial perturbation, we expand $\omega\left(k+\Delta k_{\mathrm{E}}\right)$ for small $\Delta k_{\mathrm{E}}$. Thus, the exponent results in

$$
\beta_{0}\left(\omega\left(k+\Delta k_{\mathrm{E}}\right)-\mu_{0}\right) \approx \beta_{0}\left(\omega(k)-\mu_{0}\right)+\Delta k_{\mathrm{E}} \beta_{0} \omega^{\prime}(k) .
$$

We have to compare this with the exponent in the definition of the perturbation, see Eq. (3.10). We find

$$
\begin{aligned}
\phi_{0}(k) & =\beta_{0} \Delta k_{\mathrm{E}} \omega^{\prime}(k) \\
& =-2 J \beta_{0} \Delta k_{\mathrm{E}} j^{N}(k) .
\end{aligned}
$$

Furthermore, we find that in first order in $\Delta k_{\mathrm{E}}$, the temperature has not changed after shutting down the electric field, i.e. $\beta=\beta_{0}$, and $\mu=\mu_{0}$. Including the next order in $\Delta k_{\mathrm{E}}$, we find that the temperature actually changes slightly:

$$
T_{0}=T\left(1-2 \pi^{2} \Delta k_{\mathrm{E}}^{2}\right)+\mathcal{O}\left(\Delta k_{\mathrm{E}}^{2} \varepsilon T\right) .
$$

Hence, we can safely assume that $T_{0}=\mathcal{O}(T)$. 

next-to-nearest-neighbor hopping

We need $\phi(k)$ to be small. Therefore, we have to demand that the time $\Delta t_{\mathrm{E}}$ is sufficiently small, i.e. the electric field should only be applied for a small time. Moreover, we define $\eta:=\Delta t_{\mathrm{E}} e / \hbar$, and obtain

$$
\phi_{0}(k)=\beta \eta E \omega^{\prime}(k)
$$

with $\beta \eta E=\beta \Delta t_{\mathrm{E}} e E / \hbar=\mathcal{O}(1)$. We see that the perturbation is proportional to the function $j^{N}(k)=\omega^{\prime}(k)$ of the total particle current, see Eq. (2.38). This seems to be consistent, because we applied an electric field that results in the acceleration of electrons.

Example 2: Temperature gradient: As a second example, we consider a temperature gradient. Our realization of such a gradient is preparing the initial quasiparticle momentum distribution so that the left-moving fermions have a different temperature than the right-moving ones:

$$
n_{0}(k)=\left.\theta(-k) f(k)\right|_{\beta=\beta_{1}}+\left.\theta(k) f(k)\right|_{\beta=\beta_{2}} .
$$

To calculate $\phi_{0}(k)$, we use the final temperature $T=\frac{1}{2}\left(T_{1}+T_{2}\right)$ and define the temperature difference $\Delta T=\frac{1}{2}\left(T_{2}-T_{1}\right)$. This leads to $k_{\mathrm{B}} \beta_{1}=1 /(T-\Delta T)$ and $k_{\mathrm{B}} \beta_{2}=1 /(T+\Delta T)$. We assume a small temperature difference $\Delta T$. Thus, we get

$$
\begin{aligned}
n_{0}(k) \approx & \frac{\theta(-k)}{1+\exp \left[\beta(\omega(k)-\mu)+\beta^{2} \Delta T(\omega(k)-\mu)\right]} \\
& \quad+\frac{\theta(k)}{1+\exp \left[\beta(\omega(k)-\mu)-\beta^{2} \Delta T(\omega(k)-\mu)\right]} \\
= & \frac{1}{1+\exp \left[\beta(\omega(k)-\mu)+\operatorname{sgn}(k) \beta^{2} \Delta T(\omega(k)-\mu)\right]} .
\end{aligned}
$$

We read off the corresponding perturbation:

$$
\phi_{0}(k) \approx \beta^{2} \Delta T \operatorname{sgn}(k)(\omega(k)-\mu) .
$$

It is supposed to be small. Thus, we demand for $\Delta T$ that $\beta^{2} \Delta T=\mathcal{O}(1)$ :

$$
\phi_{0}(k) \approx \beta^{2} \Delta T \operatorname{sgn}(k)(\omega(k)-\mu)
$$

It is important to note that one can choose a smoother function to replace $\operatorname{sgn}(k)$ like $\sin (2 \pi k)$ for instance. This means that one has a $k$-dependent temperature of $\beta_{0}+\beta_{0}^{2} \Delta T \sin (2 \pi k)$. One could also choose the group velocity $\omega^{\prime}(k)$, which is a sin-function with an $\varepsilon$-correction. This would mean that the initial perturbation was $\phi_{0}(k) \approx \beta_{0}^{2} \Delta T j^{Q}(k)$. Since this seems to be very artificial, we will use the example in Eq. (3.84).

Summary: Both examples lead to anti-symmetric initial perturbations $\phi_{0}(k)$. Thence, $\left\langle\phi_{0}, \chi^{(3)}\right\rangle_{\mathrm{F}}$ is non-zero. Therefore, there are long-living currents for these two initial perturbations, which we will see in Sec. 3.5.3. 


\subsection{Numerical scheme for diagonalizing the lin- earized Boltzmann equation}

In this section, we start with a description of the implementation of the numerical part of this work. We already showed how to treat the energy conservation in Sec. 3.2.3 and Eq. (3.67). First, we will introduce the discretization of the linear Boltzmann operator $\hat{\mathcal{L}}$ in Sec. 3.3.1. In App. B.5, we justify the need for a good interpolation scheme and derive the $N$-point interpolation. Sec. 3.3.2 demonstrates the finite size scaling, which is necessary due to the discretization of $\hat{\mathcal{L}}$.

The structure of the numerical algorithm is roughly:

1. Calculation of the discretized version of $\hat{\mathcal{L}}$, see Sec. 3.3.1.

2. Diagonalization of the matrix. This was achieved using algorithms from the Lapack library.

3. Extrapolation of the eigenvalues to the limit of infinitely large discretization points, see Sec. 3.3.2.

For the numerical computation, we use a rescaled version of $\hat{\mathcal{L}}$ :

$$
\hat{\mathcal{L}}^{(\mathrm{num})}[\phi](k, t):=\frac{t_{0}}{u^{2}} \hat{\mathcal{L}}[\phi](k, t),
$$

with the timescale $t_{0}=\hbar /(\pi J)$ and the dimensionless interaction $u=U / J$. By this rescaling, we obtain a dimensionless operator. Furthermore, this operator is independent of the interaction. The time scale we introduced is roughly $t_{0} \approx 0.2$ fs for $J=1 \mathrm{eV}$.

We implemented our numerical code on a computer, using the programming languages $\mathrm{C}++$ and Python. With $\mathrm{C}++$, we got the dimensionless eigenvalues $\lambda_{n}^{(\text {num })}:=t_{0} u^{-2} \lambda_{n}$ and eigenfunctions of the linearized Boltzmann operator $\hat{\mathcal{L}}^{\text {(num) }}$ for several discretizations, final chemical potentials $\mu$, final inverse temperatures $\beta$, and next-to-nearest-neighbor hoppings $\varepsilon$. Then, we used the scripting-language Python to perform finite-size scalings of the eigenvalues and to find the dependences on $\mu, \beta$, and $\varepsilon$.

\subsubsection{Calculation of the discretized linear Boltzmann op- erator}

Since a computer can only deal with a finite set of numbers, we have to choose a discretization for the linearized Boltzmann operator $\hat{\mathcal{L}}$ and the perturbation $\phi(k)$. We define the discretized momenta by

$$
\kappa_{n}=-\frac{1}{2}+\frac{n}{N_{\mathrm{k}}},
$$

with $n \in\left\{1,2, \ldots, N_{\mathrm{k}}\right\}$. With this, we discretize the perturbation $\phi(k)$ :

$$
\tilde{\phi}_{n}:=\phi\left(\kappa_{n}\right) .
$$



next-to-nearest-neighbor hopping

The objective of this section is obtaining the dimensionless discretized linearized Boltzmann operator $\mathcal{L}_{m n}$ :

$$
\frac{1}{N_{\mathrm{k}}} \sum_{n=1}^{N_{\mathrm{k}}} \mathcal{L}_{m n} \tilde{\phi}_{n} \stackrel{N_{\mathrm{k}} \rightarrow \infty}{\longrightarrow} \hat{\mathcal{L}}^{(\mathrm{num})}[\phi]\left(k_{1}\right)
$$

where $m=m_{N_{\mathrm{k}}}\left(k_{1}\right)$ is a sequence such that $\kappa_{m}$ is the discretized momentum closest to $k_{1}$. Therefore, $\kappa_{m} \rightarrow k_{1}$ in the limit $N_{\mathrm{k}} \rightarrow \infty$.

We start the derivation of $\mathcal{L}_{m n}$ by performing the integrals over $k_{2}$ and $k_{4}$ in Eq. (3.12), using the formula for the energy-conserving delta-function Eq. (3.67):

$$
\begin{aligned}
& \hat{\mathcal{L}}^{(\text {num })}[\phi]_{1} \stackrel{(3.12)}{=} \int d k_{2} d k_{3} d k_{4} \sum_{m=-1}^{1} \delta\left(\Delta k_{\vec{k}}+m\right) \delta\left(\Delta \omega_{\vec{k}}\right) \\
& \times F_{1234}\left[\phi_{1}+\phi_{2}-\phi_{3}-\phi_{4}\right] \\
& \stackrel{(3.67)}{=} \int_{-1 / 2}^{1 / 2} d k_{3} \frac{F_{1234}}{\left|\omega_{2}^{\prime}-\omega_{4}^{\prime}\right|}\left[\phi_{1}+\phi_{2}-\phi_{3}-\phi_{4}\right] \mid \begin{array}{l} 
\\
k_{2}=\tilde{k}_{2}\left(k_{1}, k_{3}\right) \\
k_{4}=k_{1}+k_{2}-k_{3}
\end{array}
\end{aligned}
$$

The remaining integral is replaced by a sum over discretized momenta:

$$
\left.\hat{\mathcal{L}}^{(\text {num })}[\phi]_{1} \approx \frac{1}{N_{\mathrm{k}}} \sum_{k_{3} \in \mathbb{D}} \frac{F_{1234}}{\left|\omega_{2}^{\prime}-\omega_{4}^{\prime}\right|}\left[\phi_{1}+\phi_{2}-\phi_{3}-\phi_{4}\right]\right|_{\substack{k_{2}=\tilde{k}_{2}\left(k_{1}, k_{3}\right) \\ k_{4}=k_{1}+k_{2}-k_{3}}} .
$$

That approximation becomes exact in the limit $N_{\mathrm{k}} \rightarrow \infty$.

Mollification: The factor $\left|\omega_{2}^{\prime}-\omega_{4}^{\prime}\right|$ vanishes for $k_{1}=k_{3}$. Obviously, at this point, the contribution to the collision term has to be zero, because $k_{1}=k_{3}$ is a trivial interaction channel, i.e. the factor $\delta_{k_{1}, \kappa_{n}}+B_{n}\left(k_{2}\right)-\delta_{k_{3}, \kappa_{n}}-B_{n}\left(k_{4}\right)$ vanishes. The numerical code, however, may not be able to account for the zero caused by this factor. Thus, we make use of the mollification procedure proposed by Fürst et al. (2012). We do it the following way:

$$
\frac{1}{\sqrt{\left(\omega_{2}^{\prime}-\omega_{4}^{\prime}\right)^{2}+\left(5 / N_{\mathrm{k}}\right)^{2}}} \stackrel{N_{\mathrm{k}} \rightarrow \infty}{\longrightarrow} \frac{1}{\left|\omega_{2}^{\prime}-\omega_{4}^{\prime}\right|} .
$$

The factor 5 is not arbitrary: We did a lot of testing, and found that for some reason the mollification number has to span at least a small number of momentum differences $1 / N_{\mathrm{k}}$.

Thence, the approximate Boltzmann operator becomes

$$
\left(\hat{\mathcal{L}}^{\text {(num) }}[\phi]_{1}\right)_{\text {approx }}=\left.\frac{1}{N_{\mathrm{k}}} \sum_{k_{3} \in \mathbb{D}} \frac{F_{1234}\left[\phi_{1}+\phi_{2}-\phi_{3}-\phi_{4}\right]}{\sqrt{\left(\omega_{2}^{\prime}-\omega_{4}^{\prime}\right)^{2}+\left(5 / N_{\mathrm{k}}\right)^{2}}}\right|_{\substack{k_{2}=\tilde{k}_{2}\left(k_{1}, k_{3}\right) \\ k_{4}=k_{1}+k_{2}-k_{3}}}
$$

It still fulfills the constraint

$$
\left(\hat{\mathcal{L}}^{(\text {num })}[\phi]_{1}\right)_{\text {approx }} \stackrel{N_{\mathrm{k}} \rightarrow \infty}{\longrightarrow} \hat{\mathcal{L}}^{(\text {num })}[\phi]_{1} .
$$


Interpolation: Now, we have to take care of the perturbation $\phi(k)$, because we only know it at the discretization points $\kappa_{n}$. The momentum $k_{3}$ is already discretized. Therefore, calculating $\phi\left(k_{3}\right)$ is not a problem. Furthermore, we choose $k_{1} \in \mathbb{D}$, so there is no problem calculating $\phi\left(k_{1}\right)$ either. However, in general, the solution $\tilde{k}_{2}\left(k_{1}, k_{3}\right)$ is not on the grid $\kappa_{n}$ for any $\varepsilon>0$. Thus, we have to choose a scheme to interpolate for the calculation of $\phi\left(\tilde{k}_{2}\right)$ and $\phi\left(k_{1}+k_{2}-k_{3}\right)$. Because simple interpolation schemes are not able to give precise results, we used the $\mathrm{N}$-point interpolation described in App. B.5. Using Eq. (B.94) from this section, we obtain

$$
\phi\left(k_{2}\right) \approx \sum_{n=1}^{N_{\mathrm{k}}} \tilde{\phi}_{n} B_{n}\left(k_{2}\right) .
$$

This equation becomes exact in the limit $N_{\mathrm{k}} \rightarrow \infty$. Plugging this into Eq. (3.90) yields

$$
\begin{aligned}
& \hat{\mathcal{L}}^{(\text {num })}[\phi]_{1} \approx \frac{1}{N_{\mathrm{k}}} \\
& \qquad \sum_{k_{3} \in \mathbb{D}} \frac{F_{1234}}{\sqrt{\left(\omega_{2}^{\prime}-\omega_{4}^{\prime}\right)^{2}+\left(5 / N_{\mathrm{k}}\right)^{2}}} \\
& \quad \times\left.\left[\phi_{1}-\phi_{3}+\sum_{n=1}^{N_{\mathrm{k}}}\left(\tilde{\phi}_{n} B_{n}\left(k_{2}\right)-\tilde{\phi}_{n} B_{n}\left(k_{4}\right)\right)\right]\right|_{\substack{k_{2}=\tilde{k}_{2}\left(k_{1}, k_{3}\right) \\
k_{4}=k_{1}+k_{2}-k_{3}}} .
\end{aligned}
$$

The next step is bringing this into a form that enables us to read off the matrix:

$$
\begin{aligned}
\hat{\mathcal{L}}^{(\text {num })}[\phi]_{1} \approx & \frac{1}{N_{\mathrm{k}}} \sum_{n=1}^{N_{\mathrm{k}}} \sum_{k_{3} \in \mathbb{D}} \frac{F_{1234}}{\sqrt{\left(\omega_{2}^{\prime}-\omega_{4}^{\prime}\right)^{2}+\left(5 / N_{\mathrm{k}}\right)^{2}}} \\
& \times\left.\left[\delta_{k_{1}, \kappa_{n}}+B_{n}\left(k_{2}\right)-\delta_{k_{3}, \kappa_{n}}-B_{n}\left(k_{4}\right)\right]\right|_{\substack{k_{2}=\tilde{k}_{2}\left(k_{1}, k_{3}\right) \\
k_{4}=k_{1}+k_{2}-k_{3}}} \tilde{\phi}_{n} \\
=: & \frac{1}{N_{\mathrm{k}}} \sum_{n=1}^{N_{\mathrm{k}}} \mathcal{L}_{m n} \tilde{\phi}_{n},
\end{aligned}
$$

with the dimensionless matrix

$$
\mathcal{L}_{m n}=\left.\sum_{k_{3} \in \mathbb{D}} \frac{F_{1234}\left[\delta_{k_{1}, \kappa_{n}}+B_{n}\left(k_{2}\right)-\delta_{k_{3}, \kappa_{n}}-B_{n}\left(k_{4}\right)\right]}{\sqrt{\left(\omega_{2}^{\prime}-\omega_{4}^{\prime}\right)^{2}+\left(5 / N_{\mathrm{k}}\right)^{2}}}\right|_{\begin{array}{l}
k_{1}=\kappa_{m} \\
k_{2}=\tilde{k}_{2}\left(k_{1}, k_{3}\right) \\
k_{4}=k_{1}+k_{2}-k_{3}
\end{array}}
$$

Thence, the final formula for the dimensionless discretized linearized Boltzmann operator is

$$
\mathcal{L}_{m n}=\left.\sum_{k_{3} \in \mathbb{D}} \frac{F_{1234}\left[\delta_{k_{1}, \kappa_{n}}+B_{n}\left(k_{2}\right)-\delta_{k_{3}, \kappa_{n}}-B_{n}\left(k_{4}\right)\right]}{\sqrt{\left(\omega_{2}^{\prime}-\omega_{4}^{\prime}\right)^{2}+\left(5 / N_{\mathrm{k}}\right)^{2}}}\right|_{\begin{array}{l}
k_{1}=\kappa_{m} \\
k_{2}=\tilde{k}_{2}\left(k_{1}, k_{3}\right) \\
k_{4}=k_{1}+k_{2}-k_{3}
\end{array}}
$$

By construction, this matrix fulfills Eq. (3.88), i.e. it describes $\hat{\mathcal{L}}^{(\text {num })}[\phi]$ in the limit $N_{\mathrm{k}} \rightarrow \infty$. 

next-to-nearest-neighbor hopping
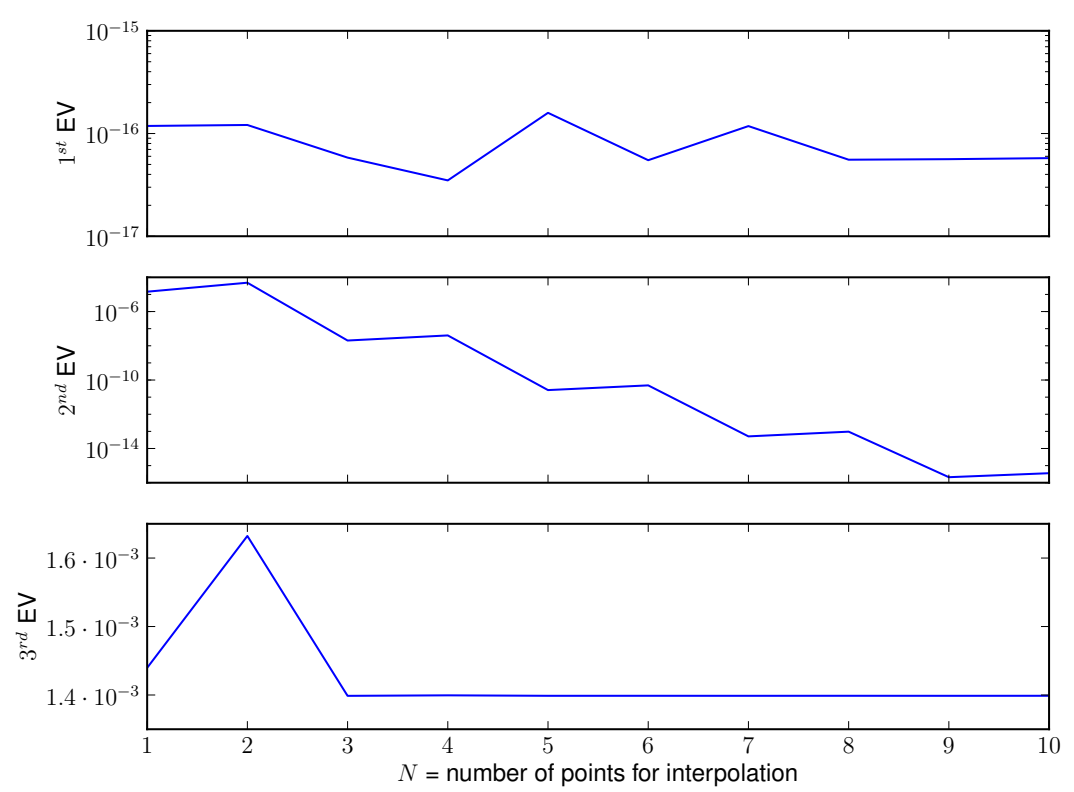

Figure 3.2: $1^{\text {st }}, 2^{\text {nd }}$, and $3^{\text {rd }}$ dimensionless eigenvalue $\lambda_{n}^{(\text {num })}=t_{0} u^{-2} \lambda_{n}$ for different $\mathrm{N}$-point interpolations.

Accuracy of the interpolation scheme: The motivation to find the accuracy of the interpolation scheme is that $\mathcal{L}_{m n}$ did not give reasonable results. We first defined test functions $\phi(k)$ and calculated $\tilde{\phi}_{n}$. Then, we compared $\frac{1}{N_{\mathrm{k}}} \sum_{n} \mathcal{L}_{m n} \tilde{\phi}_{n}$ with $\left(\hat{\mathcal{L}}^{(\text {num })}[\phi]_{1}\right)_{\text {approx }}$. We found that simple interpolation schemes were not precise enough. Only more accurate interpolation schemes give reasonably small errors.

In order to find a measure for the error, we use the fact that the first two eigenvalues have to be zero analytically. The eigenvalue $\lambda_{1}$ has the constant function as eigenfunction. Therefore, the interpolation scheme is irrelevant for this eigenvalue, which can be seen in the numerical results. There, the eigenvalue is always about 15 orders of magnitude smaller than the largest eigenvalue, see Fig. 3.2. However, for crude interpolation schemes, the second eigenvalue $\lambda_{2}$ can be 10 orders of magnitude larger, and becomes quite close to $\lambda_{3}$ for some instances. The reason is that the eigenfunction $\omega(k)$ is curved. Thus, the eigenvalue $\lambda_{2}$ measures the error from the interpolation scheme. It can be decreased by using more points for the interpolation, see Fig. 3.2.

We chose the 9-point interpolation for the whole numerical evaluation, because with this interpolation, $\lambda_{2}$ comes in the vicinity of $\lambda_{1}$. This is confirmed in Fig. 3.3, where we plot $\lambda_{1}$ and $\lambda_{2}$ for $\mu=0$ and various $\varepsilon$ and $\beta$. Both eigenvalues in Fig. 3.3 are calculated using the 9-point interpolation.

Testing the numerical code: In order to test the numerical program, we compared the results of the various algorithms at the first time step:

- "Exact": Here we calculated the Boltzmann dynamics using the exact 


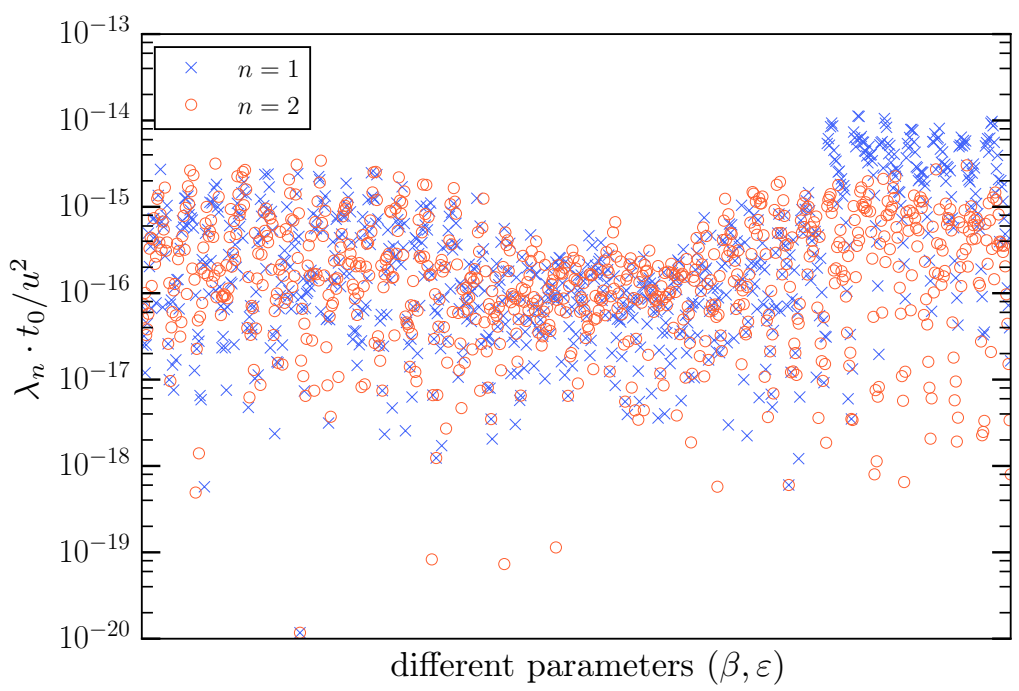

Figure 3.3: First two eigenvalues $\lambda_{1}$ and $\lambda_{2}$ for $\mu=0$, several $\varepsilon \in\left[2.5 \cdot 10^{-4}, 0.05\right]$, and several $\beta \in[0.001,40]$. They are supposed to be zero, thus the numbers in this plot can be considered the numerical error of our data.

initial quasiparticle momentum distribution $n(k, t=0)$. The advantage of this procedure is that the function can be evaluated for any momentum. Of course, this works only for the first time step. The term "exact" only refers to the exact initial quasiparticle momentum distribution. The operator is still discretized.

- "Naive": Here, we employ the discretized version of the initial function, with an approximation for the function values between two momenta. To evaluate the integral, we use the variables $k_{1}, k_{2}, k_{3}$, and $k_{4}$.

- Algorithm similar to Fürst et al. (2012): The same as above, but using relative variables $p=\frac{1}{2}\left(k_{1}-k_{2}\right)$ and $q=\frac{1}{2}\left(k_{3}-k_{4}\right)$.

- "Exact" and linearized: This procedure is similar to the "exact" scheme. The only difference is that we calculate the linearized Boltzmann operator using the exact initial perturbation $\phi(k, t=0)$.

- Linearization with N-point interpolation: Instead of the exact initial perturbation $\phi(k)$, we use an interpolation of its discretized version. The interpolation scheme uses $N$ points of the discretized perturbation $\tilde{\phi}_{n}$. The results of this scheme is closest to the exact scheme.

\subsubsection{Finite-size scaling}

Given a set of parameters $(\mu, \beta, \varepsilon)$, we calculate the discretized, linearized Boltzmann operator $\mathcal{L}_{m n}$ for different $N_{\mathrm{k}}$ up to $N_{\mathrm{k}}=2000$. We define $N_{\mathrm{k}}$ as the number of momenta on the discretization grid $\mathbb{D}$. Then, the eigenvalues are 

next-to-nearest-neighbor hopping

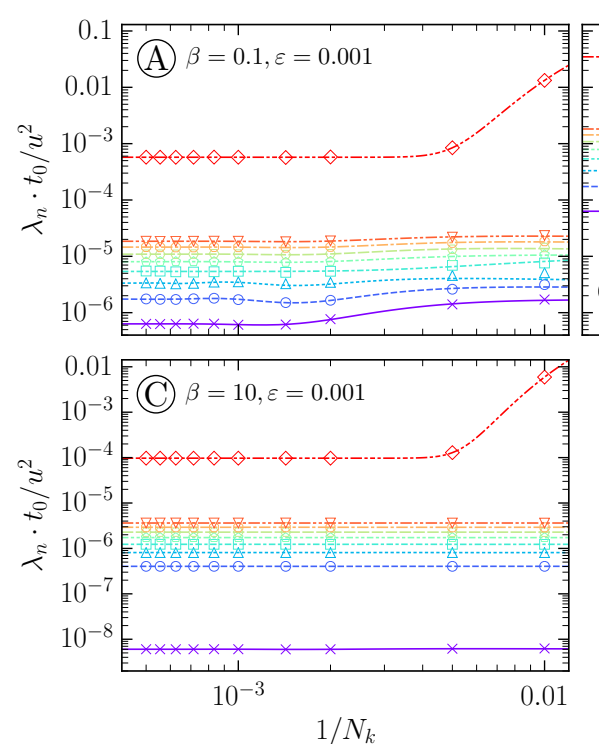

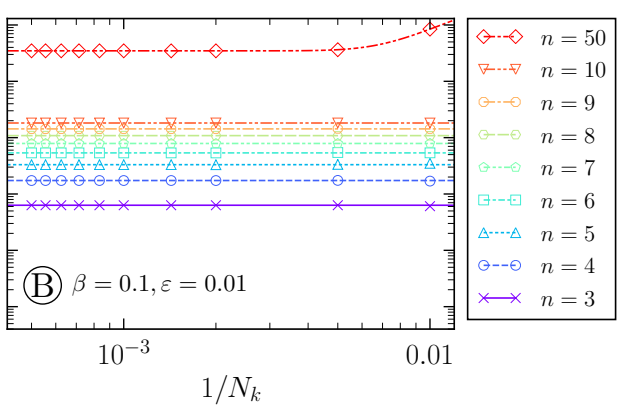

Figure 3.4: We display the interpolation to $N_{\mathrm{k}} \rightarrow \infty$ for $\mu=0$. The symbols are the data. The eigenvalue on top is $\lambda_{50}$. The lower eigenvalues are $\lambda_{3}$ till $\lambda_{10}$ from bottom to top. The lines are the fits explained in the text.

calculated and a finite-size scaling is performed with them, i.e. we extrapolate them to the limit $N_{\mathrm{k}} \rightarrow \infty$. Thereby, three cases occur (see Fig. 3.4):

1. Very small fluctuations: The convergence is so good, that the respective eigenvalue fluctuates with just a very small error, see lower eigenvalues in Figs. 3.4B and C. Here, we simply took the mean value and computed the error from the variance.

2. Exponential behavior in $1 / N_{\mathrm{k}}$ : One function is a simple exponential, see highest eigenvalue in Figs. 3.4A, B, and C.

3. Exponential decay and oscillation: At low inverse temperature $\beta$ and very low next-to-nearest-neighbor hopping $\varepsilon$ the smallest eigenvalues show a damped oscillation as a function of $1 / N_{\mathrm{k}}$, see lower eigenvalues in Fig. 3.4A. In this case, we found that the function $x \mapsto A e^{B x} \sin (C x)$ fitted very nicely.

For the fits, we gave more weight to the data with higher $N_{\mathrm{k}}$, because they are more reliable due to the fact that they lie closer to the $N_{\mathrm{k}} \rightarrow \infty$ limit.

In any case, the total error is a combination of the fit error and the error on the data points. The eigenvalues $\lambda_{1}$ and $\lambda_{2}$, which are zero analytically, fluctuate around zero in the numerical calculation due to the finite precision of the algorithm. Therefore, they can also acquire negative signs. Hence, we estimate the error of the numerical data, by the maximum of $\left|\lambda_{1}\right|$ and $\left|\lambda_{2}\right|$.

This extrapolation procedure needs to be automated, because we require the eigenvalues for a vast amount of parameter sets. Therefore, we created a script using the scripting language Python. 

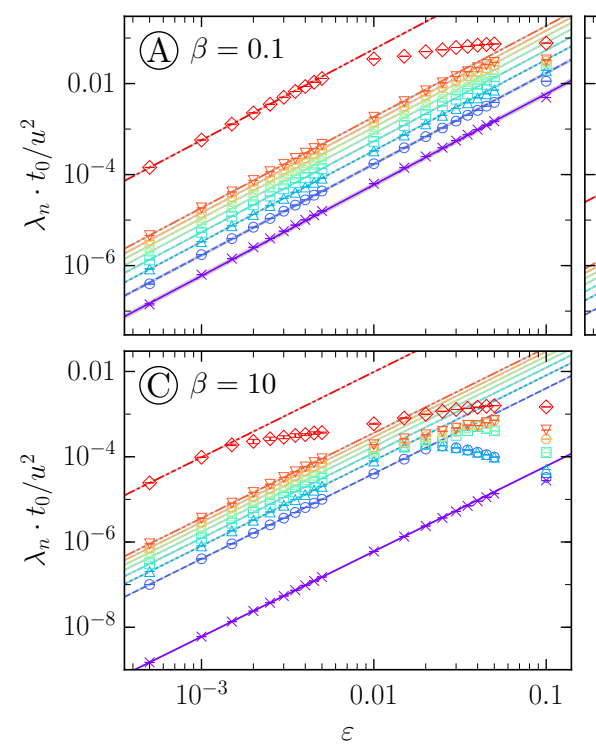

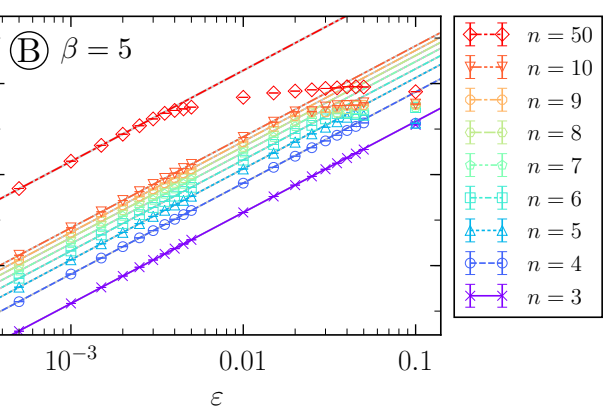

Figure 3.5: Here, we plot the eigenvalues $\lambda_{n}$ over the next-to-nearest-neighbor hopping $\varepsilon$ for different inverse temperatures $\beta$. The straight lines are fits with the function $\varepsilon \mapsto A \varepsilon^{2}$. They show that the eigenvalues are proportional to $\varepsilon^{2}$ for small $\varepsilon$.

\subsection{Results}

In the previous sections, we discussed how to diagonalize the linearized Boltzmann operator $\hat{\mathcal{L}}=\left(2 \pi U^{2} / \hbar J\right) \hat{\mathcal{L}}^{\text {(num) }}$ numerically. We showed how we computed the eigenvalues $\lambda_{n}$ of $\hat{\mathcal{L}}$. The eigenvalues are the relaxation rates of the different contributions to the perturbation $\phi(k)$ of the quasiparticle momentum distribution $n(k)$, see Eq. (3.16). In this section, we present some of the central results of this thesis, namely the behavior of the smallest relaxation rates when changing integrability breaking (next-to-nearest-neighbor hopping $\varepsilon$ ), the final temperature $\beta$, and the chemical potential $\mu$.

We plot the other eigenvalues in Fig. 3.5. There, we see double logarithmic plots with the eigenvalues' dependence on the next-to-nearest-neighbor hopping $\varepsilon$. The symbols are the eigenvalues and the lines are fits with the function $\varepsilon \mapsto \tilde{\lambda}_{n} \varepsilon^{2}$. Those lines fit the eigenvalues very well for small $\varepsilon$, thus we deduce at least for the smallest eigenvalues that

$$
\lambda_{n}=\varepsilon^{2} \tilde{\lambda}_{n} \frac{u^{2}}{t_{0}}+\mathcal{O}\left(\varepsilon^{3}\right) .
$$

This equation shows how the decay of the perturbations $\phi(k)$ changes in the regime of low $\varepsilon$.

Considering Eq. (3.99), we see that the first two coefficients in the Taylor expansion of the smallest eigenvalues $\lambda_{n}(\varepsilon)$ are zero. The expansion's zeroth order, i.e. the constant term, vanishes, because there are many zero eigenvalues for $\varepsilon=0$, which correspond to the perturbations $\phi^{S}(k)$ that are antisymmetric around $k= \pm \frac{1}{4}$, see Sec. 3.2.1. Hence, we define the low-lying eigenvalues as all the eigenvalues that vanish in the limit $\varepsilon \rightarrow 0$. In Sec. 3.6.4, we will indeed find proof that the linear part in the Taylor expansion of the low-lying eigenvalues vanishes. Thus, $\lambda_{n}=\mathcal{O}\left(\varepsilon^{2}\right)$ is numerically and will be analytically confirmed. 


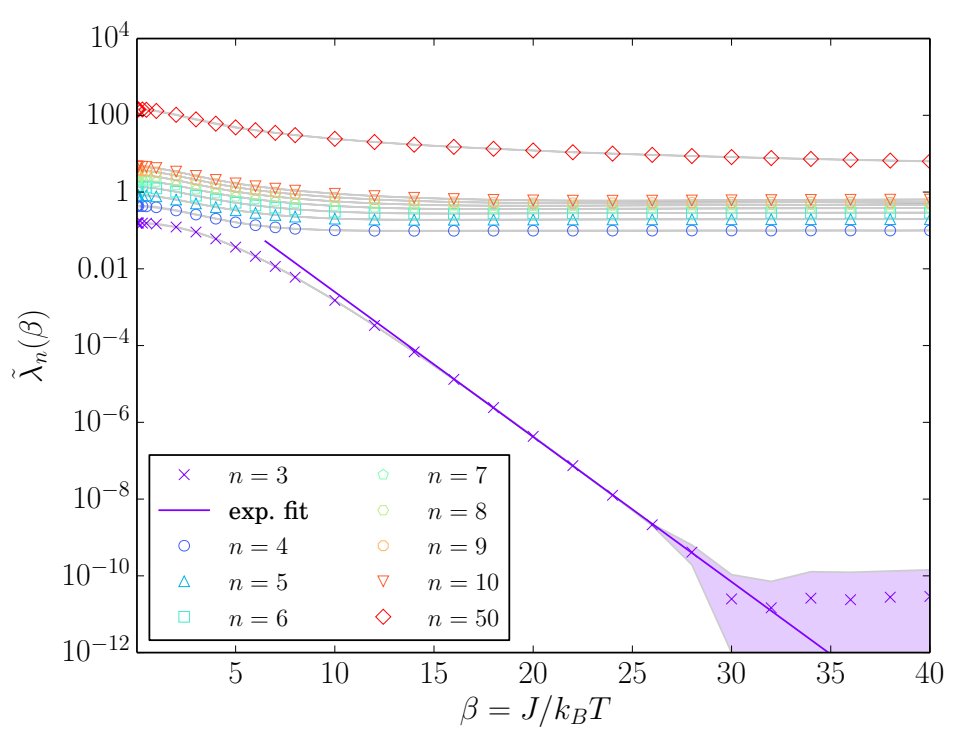

Figure 3.6: Dependence of the rescaled eigenvalues $\tilde{\lambda}_{n}$ on the final inverse temperature $\beta$. We see that only the eigenvalue $\tilde{\lambda}_{3}$ has a strong dependence on $\beta$. For high temperature, its dependence on $\beta$ is exponential.

As a next step, we make a statement about the temperature dependence of the lowest eigenvalues for small integrability breaking using the previously mentioned fits of $\lambda_{n}(\varepsilon)$ with $\varepsilon \rightarrow \tilde{\lambda}_{n} \varepsilon^{2}$, see Fig. 3.5. This is reasonable, because the fits do not only describe the small- $\varepsilon$ regime of the eigenvalues $\lambda_{n}(\varepsilon)$, they are also upper bounds on them:

$$
\lambda_{n}(\varepsilon) \leqslant \tilde{\lambda}_{n} \varepsilon^{2} .
$$

Therefore, we extract the second-order Taylor-expansion coefficient of the lowlying eigenvalues:

$$
\tilde{\lambda}_{n}:=\left.\frac{t_{0}}{2 ! u^{2}} \frac{\partial^{2}}{\partial \varepsilon^{2}} \lambda_{n}(\varepsilon)\right|_{\varepsilon=0} .
$$

In Fig. 3.6, we plot $\tilde{\lambda}_{n}$ as a function of $\beta$ with $\mu=0$. The symbols denote the data and the lines illustrate the border of the error regions. Most of the $\tilde{\lambda}_{n}$ do not vary much with inverse temperature $\beta$. Only $\tilde{\lambda}_{3}$ is different. At about $\beta=5$, it starts to decay exponentially in $\beta$. This is shown by an exponential fit, which presents itself as a line, because the plot's vertical axis is logarithmic. At the right end of the fit, there is a region of highly fluctuating values with large errors, because the values become smaller than our numerical precision, i.e. $\lambda_{3}$ cannot be distinguished from $\lambda_{1}$ and $\lambda_{2}$ within our numerical calculation. With $\varepsilon^{2} \tilde{\lambda}_{3} u^{2} / t_{0}$ being an upper bound on $\lambda_{3}$ (see Eq. (3.100)), the corresponding contribution $A_{3}(t)=A_{3}(0) e^{-\lambda_{3} t}$ to the perturbation $\phi$ can be considered conserved.

Thence, for low temperature, there is an approximately conserved quantity, i.e. a quantity that is conserved over a very long time $1 / \lambda_{3}$. This quantity is 


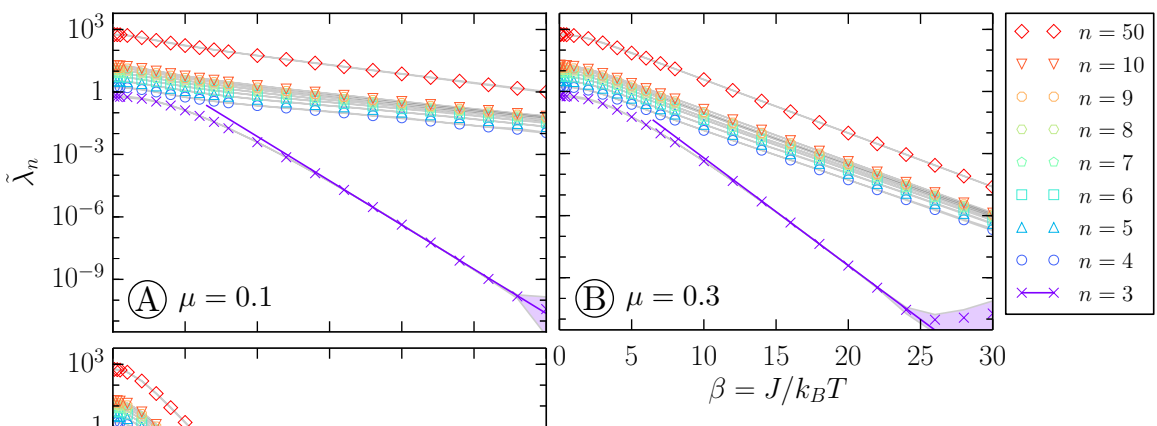

Figure 3.7: $\quad \tilde{\lambda}_{n}(\beta)$ for $\mu=0.1,0.3,0.9$ from left to right. The relaxation rates are more suppressed the further one moves away from half filling, i.e. the larger $|\mu|$. The suppression is exponential in the final inverse temperature $\beta$. Only one mode $(n=3)$ is always suppressed exponentially.

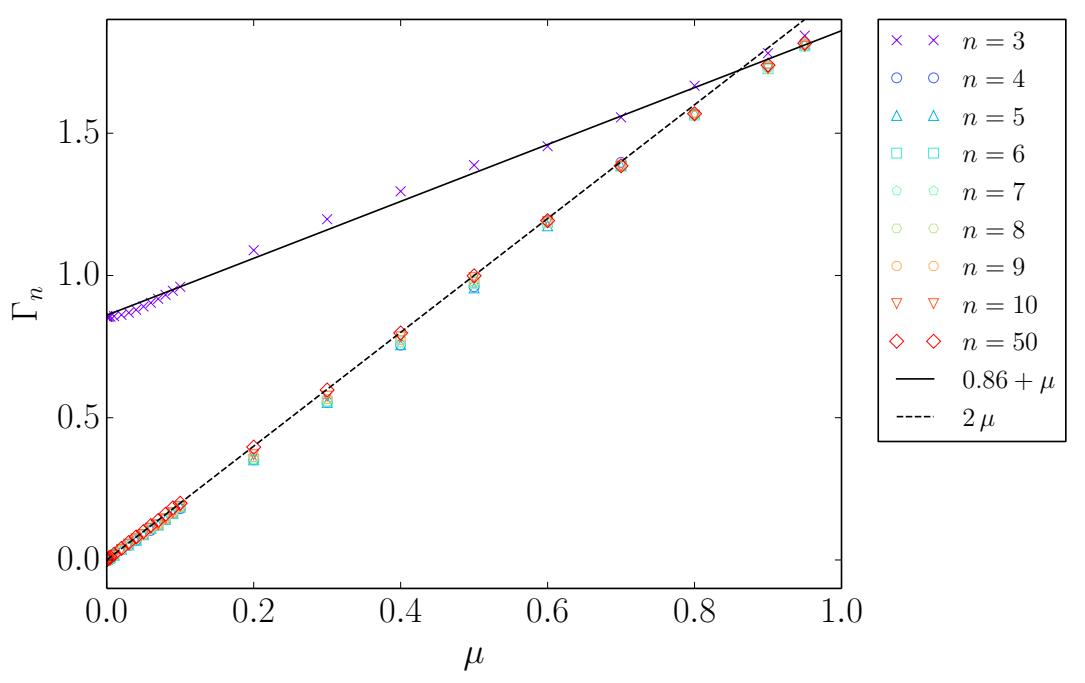

Figure 3.8: The eigenvalues decay proportional to $\exp \left[-\Gamma_{n} \beta\right]$. Here, we plot $\Gamma_{n}$. It is approximately $\Gamma_{n}=0.86+\mu$ for $n=3$ and $\Gamma_{n}=2 \mu$ for $n \geqslant 4$. 


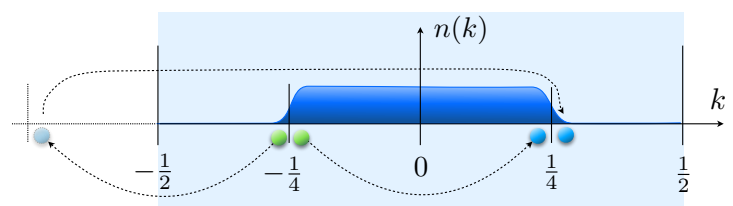

Figure 3.9: Umklapp process near the Fermi edge for $k_{\mathrm{F}} \approx \frac{1}{4}$, which means $\mu \approx 0$. A reciprocal lattice vector \pm 1 is added to the momentum of one of the fermions. Therefore, the total momentum is changed by exactly $1 / 2$. Hence, Umklapp processes at low temperature are only effective if the Fermi momentum $k_{F}$ is near $1 / 4$. Here, this means that $\mu \approx 0$, i.e. half filling.

described by the respective eigenfunction $\chi_{3}(k)$ and the corresponding statespace operator $\hat{\Psi}_{3}:=\int d k \chi_{3}(k) \hat{n}(k)$, see Sec. 2.4. In Sec. 3.5, we will give a physical meaning to this quantity and we will show that there are long-lasting currents in the systems if the initial perturbation has a contribution $A_{3}$ of the eigenvalue $\lambda_{3}$.

The eigenvalue $\lambda_{3}$ is the smallest non-zero relaxation rate. Hence, on times $t \gg 1 / \lambda_{4}$, the perturbation becomes proportional to the eigenfunction $\chi^{(3)}$, i.e.

$$
\phi(k, t) \approx\left\langle\chi^{(3)}, \phi_{0}\right\rangle_{\mathrm{F}} e^{-\lambda_{3} t} \chi^{(3)}(k),
$$

because the contributions $A_{1}(0)$ and $A_{2}(0)$ are zero, see Secs. 2.3 and 3.2.1.

So far, everything was done for a chemical potential of $\mu=0$, i.e. for half-filling. In the case of $\mu \neq 0$, we again find that $\lambda_{n}=\varepsilon^{2} \tilde{\lambda}_{n} u^{2} / t_{0}+\mathcal{O}\left(\varepsilon^{3}\right)$ (Eq. (3.99)) is still valid. The coefficient $\tilde{\lambda}_{3}$ is still exponentially suppressed as a function of $\beta$ for large $\beta$. However, the coefficients $\tilde{\lambda}_{n \geqslant 4}$ also show an exponential decay in $\beta$. For small $\mu$, there is small exponential decay, see Fig. 3.7A. For $\mu$ approaching its maximum $\left(\mu_{\max }=\omega\left(\frac{1}{2}\right)=1-\varepsilon\right)$, the exponential decay becomes as strong as the one of $\tilde{\lambda}_{3}$, see Fig. 3.7B.

We show this by fitting exponential functions $A_{n} \exp \left(x_{n}-\Gamma_{n} \beta\right)$ to the small temperature tails of $\tilde{\lambda}_{n}$ for $n \geqslant 3$. In Fig. 3.8, we illustrate the dependence $\Gamma_{n}$ on $\mu$ for $n \geqslant 3$ and $\mu \in[0,0.95]$. In good approximation, we find that

$$
\begin{aligned}
\Gamma_{3} & \approx 0.86+\mu, \\
\Gamma_{n \geqslant 4} & \approx 2 \mu .
\end{aligned}
$$

Thus, the relaxation rates are

$$
\lambda_{3} \approx \text { const } \cdot \frac{u^{2}}{t_{0}} \varepsilon^{2} e^{-\beta(0.86+|\mu|)}
$$

and

$$
\lambda_{n \geqslant 4} \approx \text { const } \cdot \frac{u^{2}}{t_{0}} \varepsilon^{2} e^{-2 \beta|\mu|}
$$

for small next-to-nearest-neighbor hopping $\varepsilon$ and for small temperature $T$. In addition, Eqs. (3.104) and (3.105) are upper bounds for the respective eigenvalues for general $\varepsilon$, see Eq. (3.100). 
We explain this behavior with the effectivity of Umklapp processes. For small temperatures, fermions can only scatter from non-empty regions of the Fermi Dirac-distribution to regions that are not fully occupied. Furthermore, energy conservation restricts these scattering processes further. For possible scattering processes, the fermions should be near the Fermi edges. At half-filling, the Fermi-edges have a distance of $\frac{1}{2}$. This is exactly the difference of the initial mean momentum and the final mean momentum of an Umklapp process, see Fig. 3.9. Thus, for $\mu=0$, Umklapp processes make the relaxation rates relatively big, even for small temperatures. However, going away from $\mu=0$, these Umklapp processes become increasingly less possible, especially for low temperature, where the Fermi edge becomes more narrow. We believe that this is why the eigenvalues $\lambda_{n} \geqslant 4$ become exponentially suppressed as a function of $\beta$ when increasing $\mu$.

Conversely, the eigenvalue $\lambda_{3}$ behaves differently than $\lambda_{n \geqslant 4}$, especially at half filling. Thus, we deduce that for $\lambda_{3}$ Umklapp processes are ineffective even for half filling.

Since the physical meaning of $\lambda_{3}$ is encoded in its corresponding eigenfunction $\chi^{(3)}(k)$, the special form of $\chi^{(3)}(k)$ may be responsible for the ineffectiveness of the Umklapp processes. In Fig. 3.10, we plot $\chi^{(3)}(k)$ for several inverse temperatures $\beta$. For high temperatures, it approaches $\omega^{\prime}(k) \omega(k)$. This function is connected to the total energy current, see Eq. (C.45). For low temperatures, the function seems to approach the saw-tooth-shaped function $k-\frac{1}{4} \operatorname{sgn}(k)$.

In order to investigate why the eigenfunction $\chi^{(3)}(k)$ has a very special eigenvalue, we compare it to the eigenfunctions $\chi^{(4,5,6)}(k)$, plotted in Fig. 3.11. They behave significantly different. Around $k=\frac{1}{4}$, the eigenfunctions $\chi^{(4,5,6)}(k)$ become flat in the low-temperature limit in contrast to $\chi^{(3)}(k)$, which obtains a large linear region in this limit. This linear region is responsible for the suppression of scattering processes for $\chi^{(3)}(k)$. We will elaborate on this in Sec. 3.5.1. There, we will define and investigate an approximate version of $\chi^{(3)}(k)$ in the zero-temperature limit.

With $\lambda_{3}$ being the smallest non-zero relaxation rate, we devote the whole Sec. 3.5 to it. Besides the already mentioned investigation of an approximate eigenfunction in Sec. 3.5.1, we will also relate a certain observable in the quantummechanical state space to $\lambda_{3}$, see Sec. 3.5.2. Moreover, in Sec. 3.5.3 we will describe the currents present on long times $t \gg 1 / \lambda_{4}$, where the perturbation $\phi(k, t)$ is described by $\chi^{(3)}(k)$.

\subsection{The first non-trivial eigenvalue of the linearized Boltzmann equation: The $3^{\text {rd }}$ eigenvalue}

In this section, we discuss the eigenvalue $\lambda_{3}$, which is the smallest non-trivial relaxation rate. In Sec. 3.5.1, we will introduce a low temperature approximation for its eigenfunction $\chi^{(3)}(k)$. Furthermore, we investigate an operator in quantum-mechanical state space, which is connected to $\lambda_{3}$, see Sec. 3.5.2. Moreover, we consider the long-time limit of operators of the form $\int d k \psi(k) \hat{n}(k)$ in 


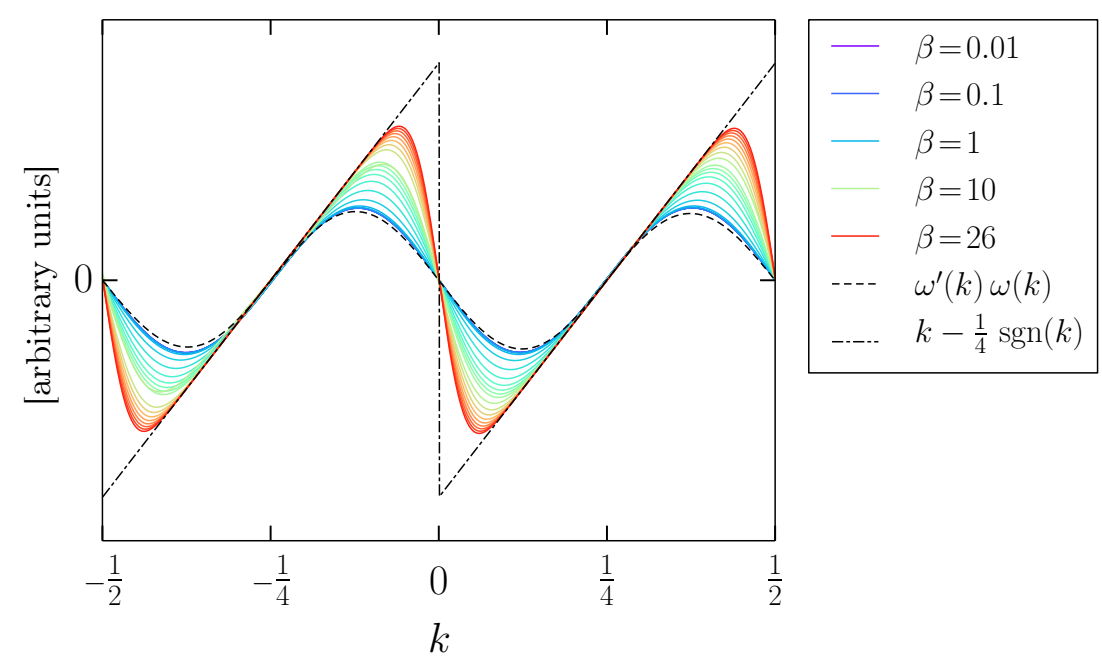

Figure 3.10: $\quad 3^{\text {rd }}$ eigenfunction for $\varepsilon=0.005$ and $L=2000$. The vertical scale is arbitrary and we chose the normalization so that the slope at $k=\frac{1}{4}$ is the same for all curves. The colored lines were found numerically and range from $\beta=0.01$ to 26. The dashed line is the function $k \mapsto A \omega^{\prime}(k) \omega(k)$. The dash-dotted line is the approximation $\tilde{\chi}^{(3)}(k) \propto k-\frac{1}{4} \operatorname{sgn}(k)$ introduced in Eq. (3.106). Thus, for low temperatures, $\chi^{(3)}(k)$ exhibits a large linear region around $k=\frac{1}{4}$. Moreover, for any temperatures, $\chi^{(3)}(k)$ is anti-symmetric around $k=\frac{1}{4}$.
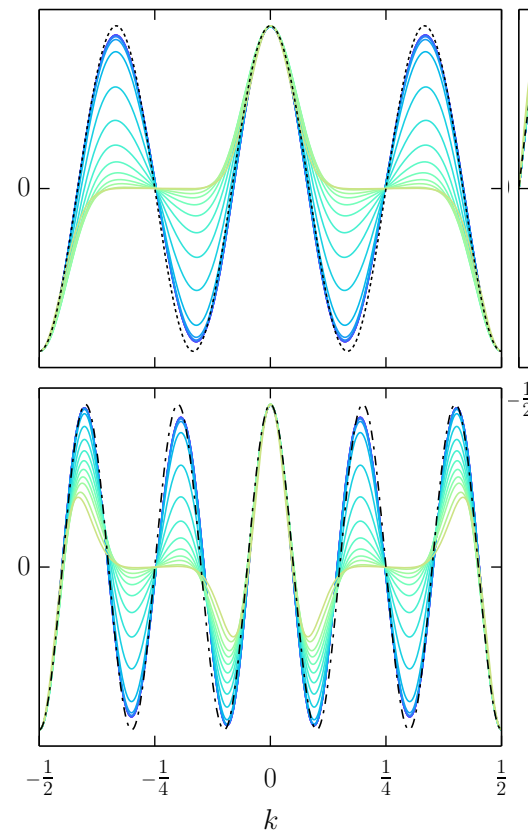

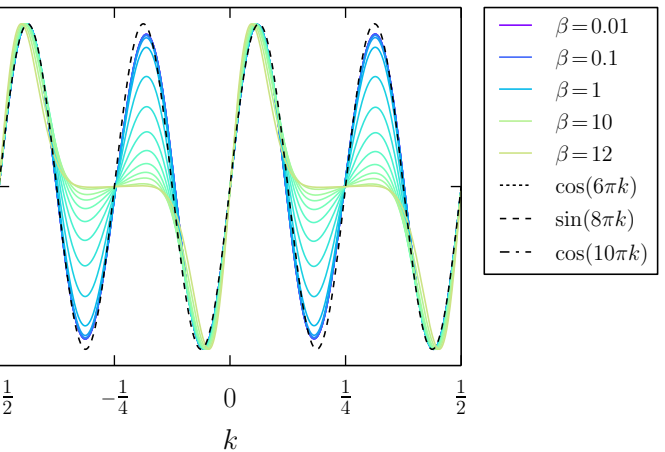

Figure 3.11: $4^{\text {th }}, 5^{\text {th }}$, and $6^{\text {th }}$ eigenfunction for $\varepsilon=0.005$ and $L=2000$. The vertical scale is arbitrary. The colored lines were found numerically and range over several values of $\beta$. The dashed lines are analytical guesses. One can clearly see that all the functions are antisymmetric around $k= \pm \frac{1}{4}$ and become very flat in that region for $T \rightarrow 0$. 


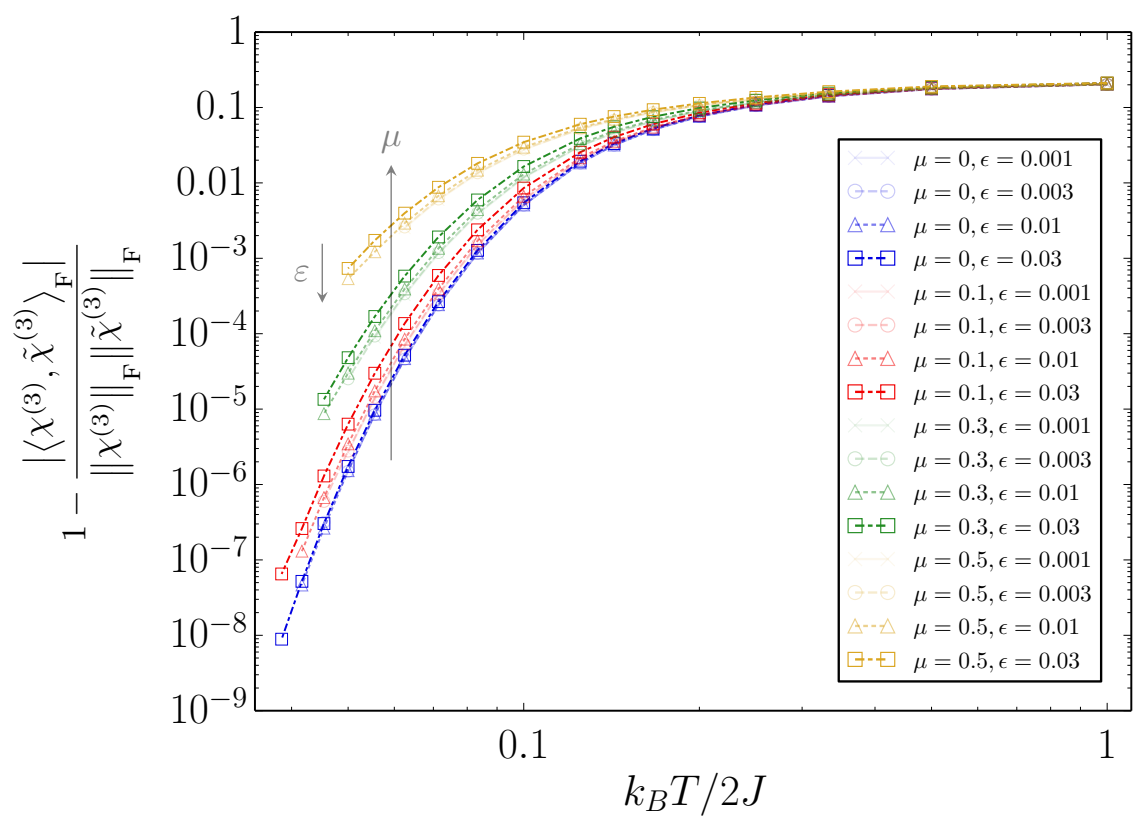

Figure 3.12: The overlap of $\chi^{(3)}(k)$ and $\tilde{\chi}^{(3)}(k)$. We plot 1 $\left|\left\langle\chi^{(3)}, \tilde{\chi}^{(3)}\right\rangle_{\mathrm{F}}\right| /\left\|\chi^{(3)}\right\|_{\mathrm{F}}\left\|\tilde{\chi}^{(3)}\right\|_{\mathrm{F}}$ as function of the dimensionless temperature $k_{\mathrm{B}} T / 2 J$. It is a good measure to see how small the overlap becomes. The plot shows that the overlap becomes small for low temperatures $T$. In this case, $\tilde{\chi}^{(3)}(k)$ is a good approximation for $\chi^{(3)}(k)$.

Sec. 3.5.3. Additionally, we present two examples for such an operator: We will calculate the expectation values of two total-current operators.

\subsubsection{The approximation of the $3^{\text {rd }}$ eigenfunction}

In this section, we introduce an approximation of the $3^{\text {rd }}$ eigenfunction $\chi^{(3)}(k)$. We will use it to determine the physical meaning of $\chi^{(3)}(k)$ by calculating the corresponding observable in the state space, which is connected in $\chi^{(3)}(k)$.

Eqs. (3.104) and (3.105) show that $\chi^{(3)}(k)$ has a different temperature dependence than the larger eigenvalues $\lambda_{n \geqslant 4}$. For $\mu=0$, it is the only eigenvalue that decreases exponentially when decreasing temperature. If $\mu \neq 0$, then all low-lying eigenvalues decrease exponentially, but $\chi^{(3)}(k)$ decays faster than the others, see Figs. 3.6 and 3.7. Hence, for sufficiently low temperature, the contribution $A_{3}(t)$ becomes approximately conserved for all $\mu$. We are interested in the physical interpretation of this conservation. To do this, we will make use of the approximate eigenfunction

$$
\tilde{\chi}^{(3)}(k):=\operatorname{kmod}(k)-\frac{1}{4} \operatorname{sgn}(\operatorname{kmod}(k))
$$



next-to-nearest-neighbor hopping

which we found in Sec. 3.4 and Fig. 3.10. The kmod function makes the $\tilde{\chi}^{(3)}(k)$ periodic. The shape of $\tilde{\chi}^{(3)}(k)$ is a saw-tooth function.

In this section, we determine the quality of the approximate eigenfunction $\tilde{\chi}^{(3)}(k)$. A natural measure for this is the scalar product $\langle\cdot, \cdot\rangle_{\mathrm{F}}$, because it is the scalar product, in which the linearized Boltzmann operator $\hat{\mathcal{L}}$ is hermitian, see Eqs. (2.22) and (B.64). Thus, for this discussion a logical choice for a quality measure of the approximation is the overlap

$$
\frac{\left|\left\langle\tilde{\chi}^{(3)}, \chi_{3}\right\rangle_{F}\right|}{\left\|\tilde{\chi}^{(3)}\right\|_{F}\left\|\chi_{3}\right\|_{F}} \text {. }
$$

The closer its value is to 1 , the better $\tilde{\chi}^{(3)}(k)$ approximates $\chi_{3}(k)$. As an example, we plot Eq. (3.107) in Fig. 3.12. Decreasing with temperature $T$, the error goes to zero, because the scalar product's factor $f(k)(1-f(k))$ gives more weight to region around the Fermi edge. The other momentum regions are exponentially suppressed by the weight factor. Therefore, according to the measure in Eq. (3.107), the approximation is very good for small temperatures, despite the fact that $\tilde{\chi}^{(3)}(k)$ does not resemble $\chi_{3}(k)$ around $k=0, \pm \frac{1}{2}$, see Fig. 3.10.

Now, we find the physical reason for the slow relaxation of the eigenfunction $\chi^{(3)}(k)$. We address this problem by using the approximate eigenfunction $\tilde{\chi}^{(3)}(k)$. The latter can provide the reason why the scattering processes are not very effective for the eigenfunction $\chi^{(3)}(k)$. In order to find it, we consider the change of the quasiparticle momentum distribution in time:

$$
\dot{n}(k, t)=f(k)(1-f(k)) \phi(k, t)=f(k)(1-f(k)) \hat{\mathcal{L}}[\phi](k, t) .
$$

Next, we assume that enough time has past that only the contribution of the $3^{\text {rd }}$ eigenfunction remains, i.e. $1 / \lambda_{4} \ll t \ll 1 / \lambda_{3}$ :

$$
\dot{n}(k, t)=A_{3}(t) f(k)(1-f(k)) \hat{\mathcal{L}}\left[\chi^{(3)}\right](k, t) .
$$

Furthermore, we replace the eigenfunction with its approximation and plug it into $\hat{\mathcal{L}}$, see Eq. (3.12):

$$
\begin{aligned}
\dot{n}_{1}=A_{3}(t) & \frac{\pi U^{2}}{\hbar J} \int d k_{2} d k_{3} \delta\left(\Delta \omega_{\vec{k}}\right) f_{1} f_{2}\left(1-f_{3}\right)\left(1-f_{4}\right) \\
\times & {\left.\left[\tilde{\chi}_{1}^{(3)}+\tilde{\chi}_{2}^{(3)}-\tilde{\chi}_{3}^{(3)}-\tilde{\chi}_{4}^{(3)}\right]\right|_{k_{4}=k_{1}+k_{2}-k_{3}}, }
\end{aligned}
$$

where we used the previously defined notation $X_{j}:=X\left(k_{j}, t\right)$. Therefore, we split the last factor into two parts:

$$
\left[\tilde{\chi}_{1}^{(3)}+\tilde{\chi}_{2}^{(3)}-\tilde{\chi}_{3}^{(3)}-\tilde{\chi}_{4}^{(3)}\right]=: K_{1234}-\Sigma_{1234},
$$

with

$$
\begin{aligned}
& K_{1234}:=\operatorname{kmod}\left(k_{1}\right)+\operatorname{kmod}\left(k_{2}\right)-\operatorname{kmod}\left(k_{3}\right)-\operatorname{kmod}\left(k_{4}\right), \\
& \Sigma_{1234}:=\frac{1}{4}\left[\operatorname{sgn}\left(\operatorname{kmod}\left(k_{1}\right)\right)+\operatorname{sgn}\left(\operatorname{kmod}\left(k_{2}\right)\right)-\operatorname{sgn}\left(\operatorname{kmod}\left(k_{3}\right)\right)-\operatorname{sgn}\left(\operatorname{kmod}\left(k_{4}\right)\right)\right] .
\end{aligned}
$$




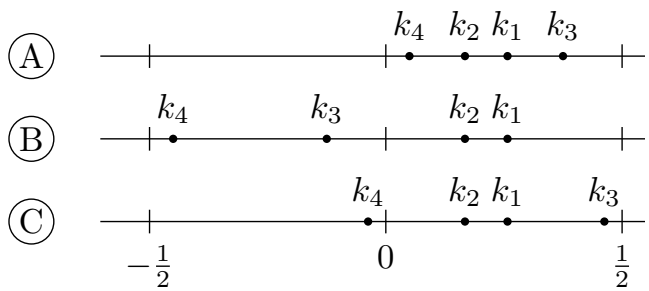

Figure 3.13: Examples for scattering processes. A: forward scattering. B: Umklapp scattering. C: Backward scattering.

We can distinguish three types of scattering processes. The first one is forward scattering, see Fig. 3.13A. All the momenta have the same sign. Here, both $K_{1234}$ and $\Sigma_{1234}$ vanish separately.

$$
\begin{aligned}
& K_{1234}=k_{1}+k_{2}-k_{3}-k_{4}=0, \\
& \Sigma_{1234}=\frac{1}{4}+\frac{1}{4}-\frac{1}{4}-\frac{1}{4}=0 .
\end{aligned}
$$

Thence, for these processes, gain and loss term balance each other out and cannot contribute to $\dot{n}(k, t)$.

The second kind is Umklapp scattering, see Fig. 3.13B. In these kind of interactions, the initial momenta have one sign and the final momenta have the opposite sign. The two terms mentioned in the first case are not zero anymore, but cancel each other out. E.g., for $k_{1}>0$, the terms become

$$
\begin{aligned}
& K_{1234}=k_{1}+k_{2}-k_{3}-\left(k_{4}-1\right)=1, \\
& \Sigma_{1234}=\frac{1}{4}+\frac{1}{4}+\frac{1}{4}+\frac{1}{4}=1 .
\end{aligned}
$$

Hence, also in this case, the processes balance each other out and do not contribute to $\dot{n}(k, t)$.

The third and last type of scattering processes is backward scattering, see Fig. 3.13C. In this case one momentum has a different sign than the other three. For the situation, depicted in the figure, said terms are

$$
\begin{aligned}
& K_{1234}=k_{1}+k_{2}-k_{3}-k_{4}=0 . \\
& \Sigma_{1234}=\frac{1}{4}+\frac{1}{4}+\frac{1}{4}-\frac{1}{4}=\frac{1}{2} .
\end{aligned}
$$

Thus, the third scattering-process type trivially conserves total momentum $\hat{K}$, but violates the difference in left and right movers. Hence, this type is the only one contributing to $\dot{n}\left(k_{1}, t\right)$. However, in this case, the factor $f_{1} f_{2}\left(1-f_{3}\right)\left(1-f_{4}\right)$ is exponentially small, because this factor only allows for processes that start in regions of the Fermi-Dirac distribution, where there are fermions, and end in regions, where fermions are missing. Note that $f_{1} f_{2}\left(1-f_{3}\right)\left(1-f_{4}\right)=(1-$ $\left.f_{1}\right)\left(1-f_{2}\right) f_{3} f_{4}$, see Eq. (B.59). Therefore, there are scattering process with $k_{1}, k_{2} \rightarrow k_{3}, k_{4}$, as well as those, where $k_{3}$ and $k_{4}$ are the initial momenta, i.e. $k_{3}, k_{4} \rightarrow k_{1}, k_{2}$. For instance, in the example of Fig. 3.13C, the momentum $k_{3}$ is near $k=\frac{1}{2}$ and $k_{4}$ near $k=0$. Hence, $\left(1-f_{3}\right)\left(1-f_{4}\right)$ is exponentially suppressed. 

next-to-nearest-neighbor hopping

The reasoning for the third scattering-process type also holds for the eigenfunctions $\chi^{(n \geqslant 4)}(k)$, whereas the types 1 and 2 do not apply to $\chi^{(n \geqslant 4)}(k)$. Hence, these two types are the reason for the difference between the low-temperature behavior of $\lambda_{3}$ and the one of $\lambda_{n} \geqslant 4$. The Umklapp and forward-scattering processes have no effect on $\tilde{\chi}^{(3)}(k)$. However, for the real eigenfunction $\chi^{(3)}(k)$, there are Umklapp and forward scattering processes that contribute, but they have to be at momenta $k_{j}=0$ or $k_{j}= \pm \frac{1}{2}$. Then, however, the factor $f_{1} f_{2}\left(1-f_{3}\right)\left(1-f_{4}\right)$ suppresses these scattering processes as in the third scattering-process type.

In this section, we introduced and justified the approximate eigenfunction $\tilde{\chi}^{(3)}(k)$. Furthermore, we explained why all the scattering processes either balance each other out or are exponentially suppressed in $\beta$.

\subsubsection{The state-space operator corresponding to the $3^{\text {rd }}$ eigenvalue}

In Sec. 2.4 we showed that the eigenfunctions $\chi^{(j)}(k)$ of the linearized Boltzmann operator $\hat{\mathcal{L}}$ are connected to the operators $\int d k \chi^{(j)}(k) \hat{n}(k)$, see Eq. (2.33). In this section, we investigate the operator corresponding to the $3^{\text {rd }}$ eigenvalue $\lambda_{3}$ :

$$
\hat{I}_{3}:=\int d k \chi^{(3)}(k) \hat{n}(k) .
$$

We will explore its physical meaning by replacing the eigenfunction with its approximation $\tilde{\chi}^{(3)}(k)=k-\frac{1}{4} \operatorname{sgn}(k)$, which we investigated in Sec. 3.5.1. This leads to

$$
\hat{I}_{3} \approx \hat{K}-\frac{1}{4}\left(\hat{N}_{\mathrm{R}}-\hat{N}_{\mathrm{L}}\right)
$$

where $\hat{K}=\int d k k \hat{n}(k)$ is the total momentum operator defined in Eq. (2.38) and it corresponds to $\phi(k)=k$. Furthermore, for Eq. (3.117), we introduced the number operator counting the fermions with positive momenta and the one counting the fermions with negative momenta:

$$
\begin{aligned}
\hat{N}_{\mathrm{R}}: & =\int_{0}^{1 / 2} d k \hat{n}(k) \\
\hat{N}_{\mathrm{L}}: & =\int_{-1 / 2}^{0} d k \hat{n}(k) .
\end{aligned}
$$

The approximation in Eq. (3.117) gives a physical meaning to the eigenfunction of the $3^{\text {rd }}$ eigenvalue $\lambda_{3}$. It is a linear composition of three simple observables. If $\hat{I}_{3}$ was equal to the total momentum, it would mean that Umklapp processes did not play a role at low temperatures. Since $\phi(k)=k$ is neither conserved for high temperature (cf. Sec. 2.3) nor for low temperature (especially for $\mu=0$, see Fig. 3.6), the expectation value of $\hat{K}$ is also not conserved, see Sec. 2.4. This means that Umklapp processes still contribute to relaxation, even at low temperatures. 
The expectation value of $\hat{I}_{3}$ is

$$
\begin{aligned}
\left\langle\hat{I}_{3}\right\rangle_{t} \stackrel{(2.37)}{=} \underbrace{\int d k \chi^{(3)} f(k)}_{=0}+\sum_{j \in \mathbb{N}} A_{j}(0) e^{-\lambda_{j} t} \underbrace{\left\langle\chi^{(3)}, \chi^{(j)}\right\rangle_{\mathrm{F}}}_{=0 \text { for } j \neq 3} \\
=A_{3}(0) e^{-\lambda_{3} t}\left\|\chi^{(3)}\right\|_{\mathrm{F}}^{2} \\
\stackrel{(2.28)}{=}\left\langle\chi^{(3)}, \phi_{0}\right\rangle_{\mathrm{F}} e^{-\lambda_{3} t} .
\end{aligned}
$$

The expectation value of $\hat{I}_{3}$ resembles the time evolution of the $3^{\text {rd }}$ eigenvalue's contribution to the perturbation $\phi(k, t)$. Hence, $\hat{I}_{3}$ is the observable connected to $\lambda_{3}$. Thus, it is an (approximately) conserved quantity for low temperatures.

There is also another way to deduce a connection between $\lambda_{3}$ and $\hat{I}_{3}$. For times $1 / \lambda_{4} \ll t \ll 1 / \lambda_{3}$, the contribution of the eigenfunction $\chi^{(3)}(k)$ can be considered to be conserved. Therefore, we apply the well-known way of finding the quasiparticle momentum distribution of the Gibbs ensemble, see for example Fetter and Walecka (2003). The only change that is made is that we add the approximately conserved quantity $\hat{I}_{3}$ to the energy and the particle number. This leads to the following partition function:

$$
Z_{\mathrm{GGE}}=\operatorname{tr} e^{-\beta \hat{H}+\beta \mu \hat{N}-\beta_{3} \hat{I}_{3}} .
$$

Since the eigenfunctions of $\hat{\mathcal{L}}$ are independent of $U$, we can consider the limit of small $U$. Therefore, the Hamiltonian is approximately equal to its kinetic part. Hence, the partition function becomes

$$
\begin{aligned}
Z_{\mathrm{GGE}} & =\sum_{\left\{n_{k}\right\}} \exp \left\{\sum_{k} n_{k}\left[-\beta \omega(k)+\beta \mu-\beta_{3} \chi^{(3)}(k)\right]\right\} \\
& =\prod_{k} \sum_{n_{k}=0}^{1} e^{n_{k}\left[-\beta \omega(k)+\beta \mu-\beta_{3} \chi^{(3)}(k)\right]} \\
& =\prod_{k}\left\{1+e^{-\beta \omega(k)+\beta \mu-\beta_{3} \chi^{(3)}(k)}\right\} .
\end{aligned}
$$

The quasiparticle momentum distribution follows from

$$
\sum_{k} n_{\mathrm{GGE}}(k)=\frac{\partial}{\partial(\beta \mu)} \ln Z_{\mathrm{GGE}}=\sum_{k} \frac{1}{1+e^{\beta(\omega(k)-\mu)+\beta_{3} \chi^{(3)}(k)}} .
$$

Therefore, we obtain

$$
\Rightarrow n_{\mathrm{GGE}}(k)=\frac{1}{1+e^{\beta(\omega(k)-\mu)+\beta_{3} \chi^{(3)}(k)}} .
$$

Comparing this to the definition of the perturbation $\phi(k)$ in Eq. (3.10), we find that the perturbation corresponding to this quasiparticle momentum distribution is

$$
\phi(k)=\beta_{3} \chi^{(3)}(k) .
$$

The coefficient $\beta_{3}$ is determined by the representation of the perturbation by the eigenfunctions of $\hat{\mathcal{L}}$ in Eq. (3.16):

$$
\beta_{3}=A_{3}(0) .
$$



next-to-nearest-neighbor hopping

This confirms the connection of the eigenfunction $\chi^{(3)}(k)$ and the operator $\hat{I}_{3}$.

In this section, we gave an example of how to relate eigenfunctions of the linearized Boltzmann operator $\hat{\mathcal{L}}$ to observables in the quantum-mechanical state space. For generic eigenfunctions, it may be very difficult to find an interpretation of the observable, especially if they have complicated peak structures, which can hardly be approximated by analytical expressions, see e.g. Fig. 4.12. We have seen that the rather simple form of the eigenfunction $\chi^{(3)}(k)$ makes the interpretation considerably easier.

\subsubsection{Long-lasting currents}

Not only can we calculate the physical quantity that is approximately conserved, but we can also test if other physical quantities do not decay for a long time. With the linearized Boltzmann equation, we can access the long-time behavior of operators of the form

$$
\hat{\Phi}[\psi]=\int d k \psi(k) \hat{n}(k),
$$

see Eq. (2.36). In this section, we study the total particle current $\hat{J}^{N}=\hat{\Phi}\left[j^{N}\right]$ and the total heat current $\hat{J}^{Q}=\hat{\Phi}\left[j^{Q}\right]$, see Eq. (2.38). The reason we concern ourselves with these current operators is the anti-symmetry of the eigenfunction $\chi^{(3)}(k)$. It suggests that a contribution from $\chi^{(3)}(k)$ to the perturbation $\phi(k)$ induces net currents. We will prove this hypothesis in the following. Also, the question arises, whether the perturbations that have a contribution from $\chi^{(3)}(k)$ are pathological or not. We will answer this question presenting the currents of the physically obvious examples from Sec. 3.2.5.

We start the investigation by considering the expectation value of $\hat{\Phi}[\psi]$ :

$$
\langle\hat{\Phi}[\psi]\rangle_{t} \stackrel{(2.36)}{=} \int d k \psi(k) f(k)+\sum_{j=3}^{\infty} A_{j}(0) e^{-\lambda_{j} t}\left\langle\chi^{(j)}, \psi\right\rangle_{\mathrm{F}} \cdot
$$

For the currents $\hat{J}^{N}$ and $\hat{J}^{Q}$, the function $\psi(k)$ is anti-symmetric. Therefore, the first term vanishes. The sum is dominated by the term with $j=3$ for long times $t \geqslant 1 / \lambda_{4}$. On these time scales, the expectation value reduces to

$$
\begin{gathered}
\langle\hat{\Phi}[\psi]\rangle_{t} \approx e^{-\lambda_{3} t} \frac{\left\langle\phi_{0}, \chi^{(3)}\right\rangle_{\mathrm{F}}\left\langle\chi^{(3)}, \psi\right\rangle_{\mathrm{F}}}{\left\|\chi^{(3)}\right\|_{\mathrm{F}}^{2}} \\
\stackrel{(3.119)}{=}\left\langle\hat{I}_{3}\right\rangle_{t} \frac{\left\langle\chi^{(3)}, \psi\right\rangle_{\mathrm{F}}}{\left\|\chi^{(3)}\right\|_{\mathrm{F}}^{2}} .
\end{gathered}
$$

Note that this expectation value is generated by a non-zero $\left\langle\phi_{0}, \chi^{(3)}\right\rangle_{\mathrm{F}}$. The factor $\left\langle\chi^{(3)}, \psi\right\rangle_{\mathrm{F}} /\left\|\chi^{(3)}\right\|_{\mathrm{F}}^{2}$ measures how different $\langle\hat{\Phi}[\psi]\rangle_{t}$ is from $\left\langle\hat{I}_{3}\right\rangle_{t}$ and how enhanced or alleviated the expectation value is compared to $\left\langle\hat{I}_{3}\right\rangle_{t}$. Thus, the expectation value $\left\langle\hat{I}_{3}\right\rangle_{t}$ generates the currents we are about to investigate and $\left\langle\chi^{(3)}, \psi\right\rangle_{\mathrm{F}} /\left\|\chi^{(3)}\right\|_{\mathrm{F}}^{2}$ can be considered as conductivities. 
Temperature dependence of expectation values: Now, we present the temperature dependence of the expectation values necessary for calculating the currents. For small temperature, the calculation can be done analytically using the Sommerfeld expansion in App. B.4 and replacing the eigenfunction $\chi^{(3)}(k)$ by its approximation $\tilde{\chi}^{(3)}(k)=k-\frac{1}{4} \operatorname{sgn}(k)$, see Sec. 3.5.1. The full calculation is presented in App. C.4.

For the total particle current $\hat{J}^{N}=\hat{\Phi}\left[j^{N}\right]$, we find the following expression in Eq. (C.12):

$$
\left\langle\tilde{\chi}^{(3)}, j^{N}\right\rangle_{\mathrm{F}}=\left\{\begin{array}{lr}
\frac{a_{0} k_{\mathrm{B}}}{4 \pi J} \arcsin (\mu-\varepsilon) T+\mathcal{O}\left(\varepsilon(\mu-\varepsilon)^{2} T\right)+\mathcal{O}\left(\varepsilon^{2} T\right)+\mathcal{O}\left(T^{3}\right) \\
\frac{a_{1} k_{\mathrm{B}}^{3}}{8 \pi J^{3}} \varepsilon T^{3}+\mathcal{O}\left(T^{5}\right) & \text { for } \varepsilon T^{2} \ll|\mu-\varepsilon| \\
\text { for }|\mu-\varepsilon| \ll \varepsilon T^{2} .
\end{array}\right.
$$

Away from half filling, the particle current is quite large, because it scales with $T$. However, for $\mu$ approaching $\varepsilon$, the prefactor becomes very small. For $\mu=\varepsilon$, which is equivalent to $k_{\mathrm{F}}=\frac{1}{4}$, there is another leading term, which scales with $T^{3}$. Note that $\mu=\varepsilon$ is approximately half filling when $\varepsilon$ is supposed to be small.

The scalar product $\left\langle\tilde{\chi}^{(3)}, j^{E}\right\rangle_{\mathrm{F}}$, which is connected to the total energy current, has different leading terms, too. Instead, we consider the heat current $\hat{J}^{Q}=$ $\hat{J}^{E}-\mu \hat{J}^{N}$. The corresponding scalar product is found in Eq. (C.14):

$$
\begin{aligned}
\left\langle\tilde{\chi}^{(3)}, j^{Q}\right\rangle_{\mathrm{F}} & =\frac{a_{1} k_{\mathrm{B}}^{3} k_{\mathrm{F}}^{\prime}}{16 J^{3}} T^{3}+\mathcal{O}\left(T^{5}\right) \\
& =\frac{a_{1} k_{\mathrm{B}}^{3}}{16 J^{3}} \frac{1}{2 \pi \sqrt{1-(\mu-\varepsilon)^{2}}} T^{3}+\mathcal{O}\left(\varepsilon^{2} T^{3}\right)+\mathcal{O}\left(\varepsilon(\mu-\varepsilon) T^{3}\right)+\mathcal{O}\left(T^{5}\right),
\end{aligned}
$$

with $j^{Q}(k)=j^{E}(k)-\mu j^{N}(k)$. We can see that it always has the same leading term regardless of the value of $\mu$.

Furthermore, the heat current is always quite small in temperature. However, it may still be larger than the particle current, because its prefactor does not scale with $\varepsilon$ or $\mu-\varepsilon$, like the one in the particle current's scalar product, see Eq. (3.129).

Temperature dependence of the currents: In the following, we investigate the total particle current $\left\langle\hat{J}^{N}\right\rangle_{t}$ and the total heat current $\left\langle\hat{J}^{Q}\right\rangle_{t}$. They have a similar temperature dependence for long times as the expectation values considered in Eqs. (3.129) and (3.130), as long as $\left\langle\phi_{0}, \chi^{(3)}\right\rangle_{\mathrm{F}} /\left\|\chi^{(3)}\right\|_{\mathrm{F}}^{2}$ is constant in the limit $T \rightarrow 0$. The elaborate calculation is presented in App. C.6.

First, we consider the total particle current using Eq. (3.128):

$$
\left\langle\hat{J}^{N}\right\rangle_{t}=e^{-\lambda_{3} t} \frac{\left\langle\phi_{0}, \chi^{(3)}\right\rangle_{\mathrm{F}}\left\langle\chi^{(3)}, j^{N}\right\rangle_{\mathrm{F}}}{\left\|\chi^{(3)}\right\|_{\mathrm{F}}^{2}}
$$

The scalar product $\left\langle\chi^{(3)}, j^{N}\right\rangle_{\mathrm{F}}$ was already presented in Eq. (3.129). In a similar fashion, we calculate the scalar products $\left\langle\phi_{0}, \chi^{(3)}\right\rangle_{\mathrm{F}}$, and $\left\langle\chi^{(3)}, \chi^{(3)}\right\rangle_{\mathrm{F}}$, see 

next-to-nearest-neighbor hopping

App. C.4 and Eqs. (C.16) and (C.18). Thus, we obtain in Eq. (C.50):

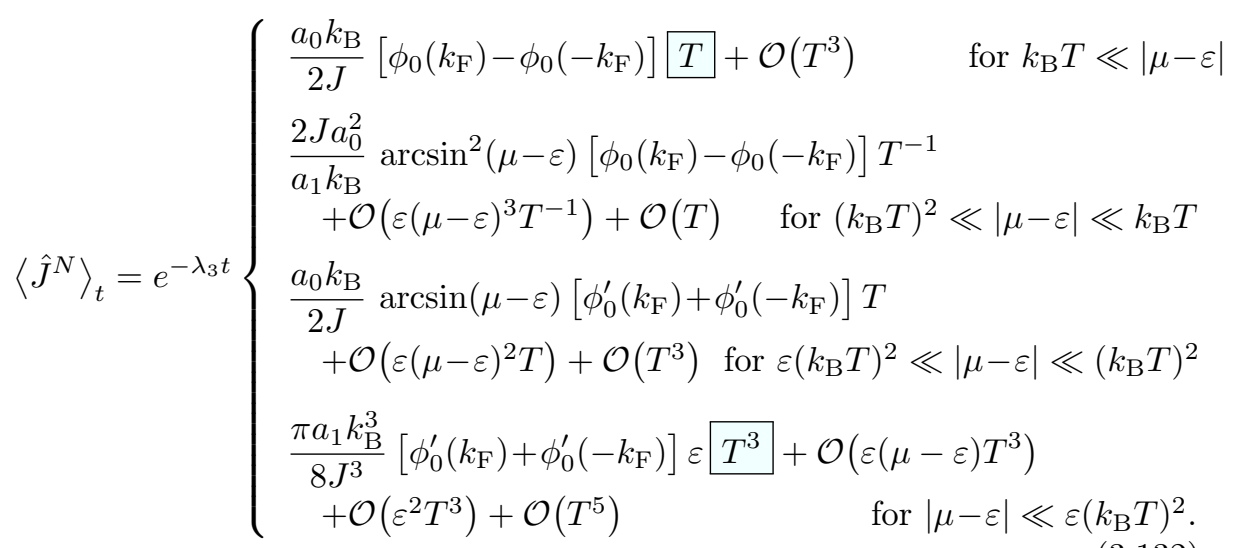

The most important regime in this equation is the first one, i.e. $k_{\mathrm{B}} T \ll|\mu-\varepsilon|$. In most cases, the temperature will, at some point, become much smaller than $|\mu-\varepsilon|$. There, the particle current is of $\mathcal{O}(T)$. For the case $\mu=\varepsilon$, this regime can never be reached. In this case, the particle current is determined by the last regime in Eq. (3.132), i.e. it is of $\mathcal{O}\left(T^{3}\right)$ for low temperatures. We will comment on the dependence on $\phi_{0}(k)$ after considering the heat current.

The expectation value of the total heat current $\hat{J}^{Q}$ can be calculated by the same computation as for the particle current. This leads to

$$
\left\langle\hat{J}^{Q}\right\rangle_{t}=\left\langle\hat{\Phi}\left[j^{Q}\right]\right\rangle_{t} \stackrel{(3.128)}{=} e^{-\lambda_{3} t} \frac{\left\langle\phi_{0}, \chi^{(3)}\right\rangle_{\mathrm{F}}\left\langle\chi^{(3)}, j^{Q}\right\rangle_{\mathrm{F}}}{\left\|\chi^{(3)}\right\|_{\mathrm{F}}^{2}} .
$$

The computation in App. C.6 leads to Eq. (C.55), which reads

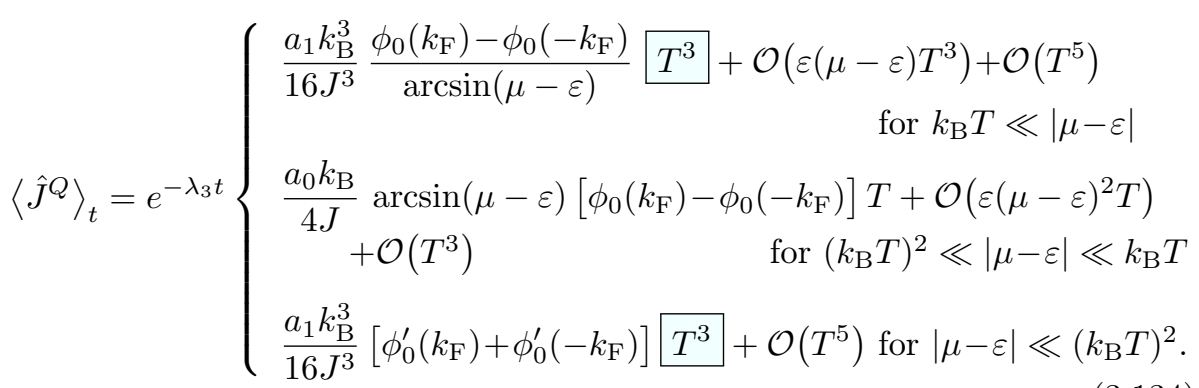

Here, the limiting regimes have the same temperature dependence, namely $T^{3}$, in contrast to the expectation value of the total particle current. Eq. (3.134) shows that the expectation value of the total energy current is proportional to $T^{3}$ if the temperature is low enough, even in the case $\mu=\varepsilon$. However, for $\mu \neq \varepsilon$, there is an intermediate regime connecting the two $T^{3}$ regimes.

For both currents, we do not consider the case $\varepsilon=0$, because there the approximation that $\lambda_{3}$ is the lowest non-zero eigenvalue is not valid any more. Furthermore, the dependencies of the factor $\phi_{0}\left(k_{\mathrm{F}}\right)-\phi_{0}\left(-k_{\mathrm{F}}\right)$ on $\varepsilon$ and $\mu$ could not be considered, because the initial perturbation $\phi_{0}(k)$ is arbitrary. If however $\phi_{0}(k)$ is symmetric around $k=0$, then both calculations give zero currents, 
because in this case, the contribution $A_{3}(0)$ of the $3^{\text {rd }}$ eigenvalue is not present in the perturbation $\phi(k, t)$ at all times. Thus, there is no current at least for times $t \gg 1 / \lambda_{4}$.

Note that the calculation of the two currents is representative of the calculation of any observable of the form $\hat{\Phi}[\psi]$. The scheme can easily be adapted to operators $\hat{\Phi}[\psi]$, as long as $\psi(k)$ and its derivatives are finite and known at $k= \pm k_{\mathrm{F}}$.

\section{Examples}

The calculation of the currents proves that there are long-lasting currents in many situations. However, it is not clear yet if these situations are just pathological. In order to address this issue, we calculate the long-lasting currents for the two initial perturbations $\phi_{0}(k)$ given in Sec. 3.2.5. Here, we present the results of the calculation in App. C.6.2. Moreover, we calculate the long-time evolution of the electrical and thermal conductivity.

Example 1: The first example is that of an electric field. We found in Sec. 3.2.5 that the initial perturbation is $\phi_{0}(k)=-(\beta \eta E / 2 J) j^{N}(k)$. In Eq. (C.64), we find that this perturbation gives the particle current

$$
\left\langle\hat{J}^{N}\right\rangle_{t}=\sigma(t) E
$$

Based on this example, we can calculate the electrical conductivity for low temperatures, and has the form

$$
\sigma(t)=e^{-\lambda_{3} t}\left\{\begin{array}{cc}
\frac{2 \pi a_{0} \eta}{\sqrt{1-(\mu-\varepsilon)^{2}}}+\mathcal{O}(\varepsilon)+\mathcal{O}\left(T^{2}\right) & \text { for } k_{\mathrm{B}} T \ll|\mu-\varepsilon| \\
\frac{8 \pi J^{2} a_{0}^{2} \eta}{a_{1} k_{\mathrm{B}}^{2}} \arcsin (\mu-\varepsilon)^{2} T^{-2}+\mathcal{O}\left(\varepsilon^{4} T^{2}\right)+\mathcal{O}\left(T^{0}\right) & \text { for } \varepsilon\left(k_{\mathrm{B}} T\right)^{2} \ll|\mu-\varepsilon| \ll k_{\mathrm{B}} T \\
\frac{4 \pi a_{1} k_{\mathrm{B}}^{2} \eta}{J^{2}} \varepsilon^{2} T^{2}+\mathcal{O}\left(\varepsilon(\mu-\varepsilon)^{3} T^{2}\right)+\mathcal{O}\left(T^{4}\right) & \text { for }|\mu-\varepsilon| \ll \varepsilon\left(k_{\mathrm{B}} T\right)^{2} .
\end{array}\right.
$$

The heat current is given in Eq. (C.68):

$$
\left\langle\hat{J}^{Q}\right\rangle_{t}=e^{-\lambda_{3} t} E\left\{\begin{array}{cc}
\frac{\pi a_{1} k_{\mathrm{B}}^{2} \eta}{4 J^{2}} \frac{1}{\arcsin (\mu-\varepsilon)} T^{2}+\mathcal{O}\left(\varepsilon T^{2}\right)+\mathcal{O}\left(T^{4}\right) & \text { for } k_{\mathrm{B}} T \ll|\mu-\varepsilon| \\
2 \pi a_{0} \eta \arcsin (\mu-\varepsilon)+\mathcal{O}\left(\varepsilon(\mu-\varepsilon)^{2}\right)+\mathcal{O}\left(T^{2}\right) & \quad \text { for } \varepsilon\left(k_{\mathrm{B}} T\right)^{2} \ll|\mu-\varepsilon| \ll k_{\mathrm{B}} T \\
\frac{\pi^{3} a_{1} k_{\mathrm{B}}^{2} \eta}{8 J^{2}} \varepsilon T^{2}+\mathcal{O}\left(\varepsilon^{2} T^{2}\right)+\mathcal{O}\left(T^{4}\right) \quad \text { for }|\mu-\varepsilon| \ll \varepsilon\left(k_{\mathrm{B}} T\right)^{2} .
\end{array}\right.
$$


Example 2: The second example is given by a temperature difference, which leads to the initial perturbation $\phi_{0}(k)=\beta^{2} \Delta T \operatorname{sgn}(k)(\omega(k)-\mu)$, see Eq. (3.84). In Eq. (C.77), we obtain the particle current

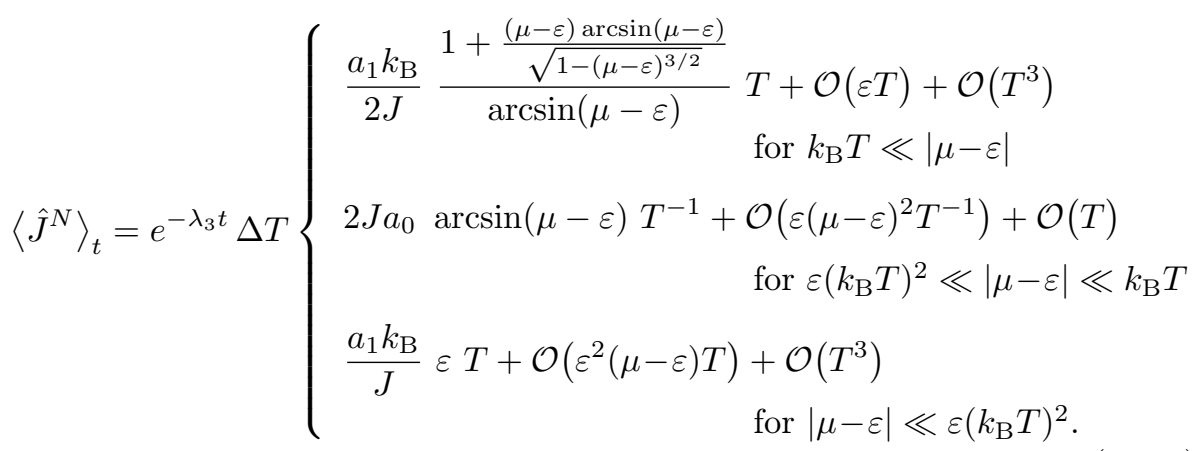

We obtain the heat current in Eq. (C.82). It is

$$
\left\langle\hat{J}^{Q}\right\rangle_{t}=\kappa \Delta T
$$

with the thermal conductivity $\kappa$ given by

$$
\kappa=e^{-\lambda_{3} t}\left\{\begin{array}{cc}
\frac{a_{1}^{2} k_{\mathrm{B}}^{3} T^{3}}{128 \pi^{3} a_{0} J^{3} \sqrt{1-(\mu-\varepsilon)^{2}}}\left[\frac{1}{1-(\mu-\varepsilon)^{2}}+\frac{\arcsin (\mu-\varepsilon)}{\sqrt{1-(\mu-\varepsilon)^{3 / 2}}}\right] \\
+\mathcal{O}\left(T^{5}\right) & \text { for } k_{\mathrm{B}} T \ll|\mu-\varepsilon| \\
\frac{a_{1} k_{\mathrm{B}}}{4 J} T+\mathcal{O}((\mu-\varepsilon) T)+\mathcal{O}\left(T^{3}\right) & \text { for }|\mu-\varepsilon| \ll k_{\mathrm{B}} T .
\end{array}\right.
$$

On the basis of this second example, we can see that the temperature dependence of the long-living currents also depends on the form of the initial perturbation $\phi_{0}(k)$. For the regime of reasonably large $\mu-\varepsilon$, the example $\phi_{0}(k) \propto \operatorname{sgn}(k)[\omega(k)-\mu]$ leads to a heat current, which is proportional to $T^{5}$, whereas the first example gives a heat current proportional to $T^{3}$. Also the particle current is different for this regime: in the first example, it is of $\mathcal{O}(T)$ and in the second it is of $\mathcal{O}\left(T^{3}\right)$.

The heat current of the first example and the particle current of the second example look very similar. Note, however, that this is not very astonishing. These, two currents would even be the same except for a constant factor if the sgn-function was replaced by $\omega^{\prime}(k)$ in the second example.

With the consideration of the two examples, we found that there are longlasting currents in realistic situations. In fact, there are many situations where currents stay in the system for a very long time. However, there are also initial perturbations, which do not lead to long-lasting currents. This is the case when $\left\langle\phi_{0}, \chi^{(3)}\right\rangle=0$. At half filling, the currents then decay on a timescale $1 / \lambda_{4} \ll 1 / \lambda_{3}$ or faster. Away from half filling, we have seen in Fig. 3.7 that all low-lying eigenvalues decay exponentially in inverse temperature. In this case, one can at least say that initial perturbations $\phi_{0}(k)$, that are symmetric around $k= \pm \frac{1}{4}$, lead to $\left\langle\phi_{0}, \chi^{(n \geqslant 3)}\right\rangle=0$ (see Figs. 3.10 and 3.11 ) for all low-lying eigenvalues $\lambda_{n} \geqslant 3$. Hence, with this symmetry, $\phi_{0}$ is expected to decay relatively fast away from half filling. 
In this section, we have seen how to calculate currents, especially long-lasting currents, in the system by means of the linearized Boltzmann equation. This kind of investigation is exemplary for other observables of the form $\int d k \psi(k) \hat{n}(k)$ that might be of interest. We showed that we can calculate the time evolution of their expectation values and their dependence on the final temperature in the case of long times and small temperatures. Note that this calculation for long times is rather simple, because there is only one non-zero relaxation rate $\lambda_{3}$, that is much smaller than the others. For other models, there can be a vast amount of low relaxation rates, which are of the same order of magnitude, which means that many relaxation rates have to be considered for the long-time behavior of currents and other physical quantities. Examples for such models include this model close to complete or empty filling (see Fig. 3.7) or the one-dimensional manganite model presented in Fig. 4.11.

\subsection{Perturbation theory of the linearized Boltz- mann operator}

This section is concerned with the perturbation theory of the linear operator $\hat{\mathcal{L}}$ for small $\varepsilon$. We will show that the first order vanishes. The second order perturbation theory is complicated by the fact that the eigenvalue zero is infinitely degenerate. Thus, when calculating the second-order approximation, one would have to diagonalize an infinite-dimensional matrix. Therefore, we will not calculate it, but only use the fact of the vanishing first order to explain the numerical results in Sec. 3.4.

We will first reformulate $\hat{\mathcal{L}}$ from Eq. (3.12). The momenta $k_{1}, k_{2}, k_{3}$, and $k_{4}=$ $k_{1}+k_{2}-k_{3}$ will be replaced with the variables $k, p$, and $q$ defined in Eq. (3.25). We use the latter, because they seem to be natural for scattering processes, since $k$ measures the conserved total momentum. Furthermore, introducing these variables, $\Delta \omega_{\vec{k}}=0$ factors into the trivial solution and the non-trivial solution, see Eq. (3.28).

\subsubsection{Center of mass and relative momentum coordinates}

A Fourier basis is utilized to map the integral operator to an infinite matrix. We choose this basis so that half of it is in $\operatorname{ker} \hat{\mathcal{L}}^{\varepsilon=0}$ and the others are orthogonal to all functions in $\operatorname{ker} \hat{\mathcal{L}}^{\varepsilon=0}$. This will be used to perform the perturbation theory later on. The basis functions in $\operatorname{ker} \hat{\mathcal{L}}^{\varepsilon=0}$ are

$$
v_{n}(k):=\sqrt{2} \sin \left(2 \pi n\left(k-\frac{1}{4}\right)\right), \quad \forall n \in \mathbb{N} .
$$

They are antisymmetric around $k= \pm \frac{1}{4}$, like the perturbations of the stationary states in Eq. (3.20). The other functions are

$$
w_{n}(k):=\sqrt{2} \cos \left(2 \pi n\left(k-\frac{1}{4}\right)\right), \quad \forall n \in \mathbb{N}_{0} .
$$

Thus, the set of eigenfunctions with eigenvalue zero has an infinitely large basis, and so does the set of the remaining eigenfunctions. 
We can determine the relative degeneracy of the eigenvalue zero. In order to do this, we first put a cut-off $|n| \leqslant N$ on the wavelength $2 \pi n$ of the basis functions. Hence, the $N+1$ functions $v_{n}(k)$ have eigenvalue zero. Furthermore, there are $N$ remaining functions $w_{n}(k)$. The relative degeneracy is

$$
\frac{N+1}{2 N+1} \stackrel{N \rightarrow \infty}{\longrightarrow} \frac{1}{2}
$$

Thus, half the eigenfunctions of $\hat{\mathcal{L}}$ have eigenvalue zero.

In Eq. (3.12), we saw that the operator $\hat{\mathcal{L}}$ of the linearized Boltzmann equation acting on a perturbation $\phi$ has the form

$$
\begin{gathered}
\hat{\mathcal{L}}[\phi]_{1}=\frac{\pi U^{2}}{\hbar J} \int d k_{2} d k_{3} d k_{4} \sum_{m=-1}^{1} \delta\left(\Delta k_{\vec{k}}+m\right) \delta\left(\Delta \omega_{\vec{k}}\right) \\
\times \frac{1-f_{2}}{f_{1}} f_{3} f_{4}\left[\phi_{1}+\phi_{2}-\phi_{3}-\phi_{4}\right] .
\end{gathered}
$$

It is convenient to use relative momenta $p, q$ and half total momenta $k, k^{\prime}$ before and after the collision instead of $k_{1}, \ldots, k_{4}$. The transformation to from the former to the latter reads

$$
k_{1}=k+p, \quad k_{2}=k-p, \quad k_{3}=k^{\prime}+q, \quad k_{4}=k^{\prime}-q .
$$

These can be used to replace the integration variables $k_{1,2,3,4}$ :

$$
\int d^{4} k \delta\left(\Delta k_{\vec{k}}+m\right) \ldots=\left|\operatorname{det}\left(\begin{array}{cc}
1 & 1 \\
1 & -1
\end{array}\right)\right|_{\left[-\frac{1}{2}, \frac{1}{2}\right]^{4}}^{2} \int_{k} d k d k^{\prime} d p d q \delta\left(2 k-2 k^{\prime}\right) \ldots
$$

Applying the momentum conservation $k=k^{\prime}$, we obtain

$$
\int d^{4} k \delta\left(\Delta k_{\vec{k}}+m\right) \ldots=2 \int_{\left[-\frac{1}{2}, \frac{1}{2}\right]^{3}} d k d p d q \ldots
$$

In the calculation of the operator $\hat{\mathcal{L}}$, there is no integral over $k_{1}$. However, we can add an integral $\int d k_{1}^{\prime} \delta\left(k_{1}^{\prime}-k_{1}\right)$ and get

$$
\begin{aligned}
\hat{\mathcal{L}}[\phi]=-\frac{2 \pi U^{2}}{\hbar J} & \int d k d p d q \delta\left(k+p-k_{1}\right) \delta\left(\Delta \omega_{k, p, q}^{\varepsilon}\right) \frac{1-f_{p-}}{f_{p+}} f_{q+} f_{q-} \\
& \times\left[\phi_{p+}, \frac{1}{2}\right]^{3} \\
& \left.\times \phi_{p-}-\phi_{q+}-\phi_{q-}\right]
\end{aligned}
$$

where we introduced the index notation $g_{p \pm}:=g(k \pm p)$. In the next section, we will perform the basis transformation and consider the energy-conserving delta-function $\delta\left(\Delta \omega_{\vec{k}}\right)$. 


\subsubsection{Basis transformation}

We write the Boltzmann operator $\hat{\mathcal{L}}$ in the following Fourier basis

$$
\zeta_{n}(k)=\bar{\vartheta}_{n} v_{|n|}(k)+\vartheta_{n} w_{n}(k), \quad n \in \mathbb{Z},
$$

with

$$
\begin{aligned}
& \vartheta_{n}:= \begin{cases}1 & \text { if } n \geqslant 0 \\
0 & \text { else }\end{cases} \\
& \bar{\vartheta}_{n}:=1-\vartheta_{n}
\end{aligned}
$$

and the definitions in Eqs. (3.141) and (3.142) of $v_{n}$ and $w_{n}$, respectively. $\zeta_{0}$ is the constant function, $\zeta_{n \geqslant 0}$ are eigenfunctions with eigenvalue zero for $\varepsilon=0$, and the $\zeta_{n<0}$ are the other Fourier modes.

In this way, the operator $\hat{\mathcal{L}}$ maps into an infinite matrix

$$
\begin{aligned}
\mathcal{L}_{m n}^{\varepsilon}:= & \left\langle\zeta_{m}, \hat{\mathcal{L}}\left[\zeta_{n}\right]\right\rangle_{\mathrm{F}} \\
=- & \frac{2 \pi U^{2}}{\hbar J} \int_{\left[-\frac{1}{2}, \frac{1}{2}\right]^{3}} d k d p d q \zeta_{m, p+} \delta\left(\Delta \omega_{\vec{k}}\right)\left[1-f_{p+}\right]\left[1-f_{p-}\right] f_{q+} f_{q-} \\
& \times\left[\zeta_{n, p+}+\zeta_{n, p-}-\zeta_{n, q+}-\zeta_{n, q-}\right] .
\end{aligned}
$$

We now replace the energy conservation with

$$
\delta\left(\Delta \omega_{k, p, q}^{\varepsilon}\right) \stackrel{(3.74)}{=} \frac{1}{4 \pi} \frac{\gamma\left(\varepsilon r_{p q}\right)}{\left|c_{p}-c_{q}\right|}\left\{\delta\left(k-\tilde{k}_{p q}^{\varepsilon}\right)+\delta\left(k+\tilde{k}_{p q}^{\varepsilon}\right)\right\}
$$

Thence, we obtain

$$
\begin{aligned}
\mathcal{L}_{m n}^{\varepsilon}=- & \frac{U^{2}}{2 \hbar J} \int d k d p d q \zeta_{m, p+} \frac{\gamma\left(\varepsilon r_{p q}\right)}{\left|c_{p}-c_{q}\right|}\left\{\delta\left(k-\tilde{k}_{p q}^{\varepsilon}\right)+\delta\left(k+\tilde{k}_{p q}^{\varepsilon}\right)\right\} \\
& \times\left[1-f_{\left.p+\frac{1}{2}, \frac{1}{2}\right]^{3}}\right]\left[1-f_{p-}\right] f_{q+} f_{q-}\left[\zeta_{n, p+}+\zeta_{n, p-}-\zeta_{n, q+}-\zeta_{n, q-}\right]
\end{aligned}
$$

with the index notation $g_{p \pm}:=g(k \pm p)$. To evaluate $\delta\left(k+\tilde{k}_{p q}^{\varepsilon}\right)$, we need to know how $\zeta_{n}$ transforms under $k \rightarrow-k$ :

$$
\begin{aligned}
\zeta_{n} & (-k)=\mathcal{N}_{n}\left[\bar{\vartheta}_{n} \sin \left(2 \pi|n|\left(-k-\frac{1}{4}\right)\right)+\vartheta_{n} \cos \left(2 \pi n\left(-k-\frac{1}{4}\right)\right)\right] \\
& =\mathcal{N}_{n}\left[-\bar{\vartheta}_{n} \sin \left(2 \pi|n|\left(k-\frac{1}{4}\right)+\pi|n|\right)+\vartheta_{n} \cos \left(2 \pi n\left(k-\frac{1}{4}\right)+\pi n\right)\right] \\
& =\mathcal{N}_{n}\left[(-1)^{n+1} \bar{\vartheta}_{n} \sin \left(2 \pi|n|\left(k-\frac{1}{4}\right)+\pi|n|\right)+(-1)^{n} \vartheta_{n} \cos \left(2 \pi n\left(k-\frac{1}{4}\right)+\pi n\right)\right] \\
& =(-1)^{n+\bar{\vartheta}_{n}} \zeta_{n}(k) .
\end{aligned}
$$

Moreover, we notice that

$$
\left.g\left(\tilde{k}_{p, q}^{\varepsilon}+p\right)\right|_{p \rightarrow-p}=g\left(\tilde{k}_{-p, q}^{\varepsilon}-p\right) \stackrel{(3.43)}{=} g\left(\tilde{k}_{p q}^{\varepsilon}-p\right)=g\left(\tilde{k}_{p, q}^{\varepsilon}-p\right)
$$

for any function $g$, because $\tilde{k}_{p q}^{\varepsilon}$ only depends on $\varepsilon r_{p q}=\varepsilon\left(c_{p}+c_{q}\right)$. Continuing the calculation of the matrix elements, we additionally use the symmetries of 
$f_{q \pm}$ and $\zeta_{n, p+}+\zeta_{n, p-}-\zeta_{n, q+}-\zeta_{n, q-}$ to obtain next-to-nearest-neighbor hopping

$$
\begin{aligned}
\mathcal{L}_{m n}^{\varepsilon}=-\frac{U^{2}}{2 \hbar J} & \int_{\left[-\frac{1}{2}, \frac{1}{2}\right]^{2}} d p d q \frac{\gamma\left(\varepsilon r_{p q}\right)}{\left|c_{p}-c_{q}\right|}\left\{\zeta_{m, \tilde{p}+}+(-1)^{m+\bar{\vartheta}_{m}+n+\bar{\vartheta}_{n}} \zeta_{m, \tilde{p}-}\right\} \\
& \left.\times\left[1-f_{\tilde{p}+}\right]\left[1-f_{\tilde{p}-}\right] f_{\tilde{q}+} f_{\tilde{q}-}\left[\zeta_{n, \tilde{p}+}+\zeta_{n, \tilde{p}-}-\zeta_{n, \tilde{q}+}-\zeta_{n, \tilde{q}-}\right]\right\}
\end{aligned}
$$

We continue with changing the integration over $p$ to one over $-p$ in the term including $(-1)^{m+\bar{\vartheta}_{m}+n+\bar{\vartheta}_{n}} \zeta_{m, \tilde{p}-}$ :

$$
\begin{aligned}
\mathcal{L}_{m n}^{\varepsilon}=-\frac{U^{2}}{\hbar J} & \Delta_{n+m+\bar{\vartheta}_{n}+\bar{\vartheta}_{m}} \int_{\left[-\frac{1}{2}, \frac{1}{2}\right]^{2}} d p d q \frac{\gamma\left(\varepsilon r_{p q}\right)}{\left|c_{p}-c_{q}\right|} \zeta_{m, \tilde{p}+}\left[1-f_{\tilde{p}+}\right]\left[1-f_{\tilde{p}-}\right] f_{\tilde{q}+} f_{\tilde{q}-} \\
& \times\left[\zeta_{n, \tilde{p}+}+\zeta_{n, \tilde{p}-}-\zeta_{n, \tilde{q}+}-\zeta_{n, \tilde{q}-}\right]
\end{aligned}
$$

where we introduced $g_{\tilde{p} \pm}=g\left(\tilde{k}_{p q}^{\varepsilon} \pm p\right)$. Furthermore, we changed the integration variable $p$ to $-p$ in the term including $\zeta_{m, \tilde{p}-}$. Additionally, in the last line we defined

$$
\Delta_{l}:=\frac{1}{2}\left(1+(-1)^{l}\right) \in 0,1 .
$$

Thence, the factor $\Delta_{n+m+\bar{\vartheta}_{n}+\bar{\vartheta}_{m}}$ makes half the matrix elements vanish.

\subsubsection{Kernel of the linearized operator in the integrable case}

After we performed the basis transformation of $\hat{\mathcal{L}}$ to $\mathcal{L}_{m n}^{\varepsilon}$ in the last section, we continue with the search for a basis of $\operatorname{ker}(\hat{\mathcal{L}})$, i.e. the eigenfunctions of $\hat{\mathcal{L}}$ with eigenvalue zero. Using $\gamma(0)=1$ (Eq. (3.73)), the matrix $\mathcal{L}_{m n}^{\varepsilon}$ in Eq. (3.157) attains the form

$$
\begin{aligned}
\mathcal{L}_{m n}^{0}=- & \frac{U^{2}}{\hbar J} \Delta_{n+m+\bar{\vartheta}_{n}+\bar{\vartheta}_{m}} \int d p d q \frac{\zeta_{m}\left(\frac{1}{4}+p\right)}{\left|c_{p}-c_{q}\right|}\left[1-f^{0}\left(\frac{1}{4}+p\right)\right]\left[1-f^{0}\left(\frac{1}{4}-p\right)\right] \\
& \times f^{0}\left(\frac{1}{4}+q\right) f^{0}\left(\frac{1}{4}-q\right)\left[\zeta_{n}\left(\frac{1}{4}+p\right)+\zeta_{n}\left(\frac{1}{4}-p\right)-\zeta_{n}\left(\frac{1}{4}+q\right)-\zeta_{n}\left(\frac{1}{4}-q\right)\right] .
\end{aligned}
$$

Furthermore, we plug in the basis $\zeta_{n}(k)$ defined in Eq. (3.149):

$$
\begin{aligned}
\mathcal{L}_{m n}^{0}= & -\frac{U^{2} \Delta_{n+m+\bar{\vartheta}_{n}+\bar{\vartheta}_{m}}}{\hbar J} \int d p d q \frac{\left[1-f^{0}\left(\frac{1}{4}+p\right)\right]\left[1-f^{0}\left(\frac{1}{4}-p\right)\right] f^{0}\left(\frac{1}{4}+q\right) f^{0}\left(\frac{1}{4}-q\right)}{\left|c_{p}-c_{q}\right|} \\
& \times\left[\bar{\vartheta}_{m} \sin (2 \pi|m| p)+\vartheta_{m} \cos (2 \pi m p)\right]\left[2 \vartheta_{n} \cos (2 \pi n p)-2 \vartheta_{n} \cos (2 \pi n q)\right] .
\end{aligned}
$$

Next, we make use of the fact that the term including $\sin (2 \pi|m| p)$ is odd in $p$ and therefore vanishes:

$$
\begin{aligned}
\mathcal{L}_{m n}^{0}=- & \frac{2 U^{2}}{\hbar J} \vartheta_{m} \vartheta_{n} \Delta_{m+n} \int_{\left[-\frac{1}{2}, \frac{1}{2}\right]^{2}} d p d q \frac{c_{n p}-c_{n q}}{\left|c_{p}-c_{q}\right|} c_{m p} \\
& \times\left[1-f^{0}\left(\frac{1}{4}+p\right)\right]\left[1-f^{0}\left(\frac{1}{4}-p\right)\right] f^{0}\left(\frac{1}{4}+q\right) f^{0}\left(\frac{1}{4}-q\right) .
\end{aligned}
$$


Now, we symmetrize the $p$ - and $q$-dependent integrand. This is possible since $\left[1-f_{1}\right]\left[1-f_{2}\right] f_{3} f_{4}=f_{1} f_{2}\left[1-f_{3}\right]\left[1-f_{4}\right]$, see Eq. (B.59). We obtain

$$
\begin{aligned}
\mathcal{L}_{m n}^{0}=- & \frac{U^{2}}{\hbar J} \vartheta_{m} \vartheta_{n} \Delta_{m+n} \int_{\left[-\frac{1}{2}, \frac{1}{2}\right]^{2}} d p d q \frac{\left(c_{n p}-c_{n q}\right)\left(c_{m p}-c_{m q}\right)}{\left|c_{p}-c_{q}\right|} \\
& \times\left[1-f^{0}\left(\frac{1}{4}+p\right)\right]\left[1-f^{0}\left(\frac{1}{4}-p\right)\right] f^{0}\left(\frac{1}{4}+q\right) f^{0}\left(\frac{1}{4}-q\right) .
\end{aligned}
$$

In the case of $n \leqslant 0$, the matrix elements are zero. For $n<0$, the reason is the antisymmetry of $v_{n}(k)$ around $k= \pm \frac{1}{4}$ leading to the factor $\bar{\vartheta}_{n}$. For $n=0$, the factor $w_{0}(k+p)+w_{0}(k-p)-w_{0}(k+q)-w_{0}(k-q)=0$ leads to the factor $c_{n p}-c_{n q}=0$. Therefore, the associated $\zeta_{n \leqslant 0}$ are eigenfunctions of $\hat{\mathcal{L}}^{\varepsilon=0}$ with eigenvalue zero, see Eq. (3.141). Hence, we have

$$
v_{n} \in \operatorname{ker} \hat{\mathcal{L}}^{\varepsilon=0} \quad \forall n \leqslant 0
$$

with $v_{n}(k)=\sqrt{2} \sin \left(2 \pi n\left(k-\frac{1}{4}\right)\right)$, cf. Eq. (3.141). The first two eigenfunctions $\zeta_{0}(k)=1$ and $\zeta_{-1}(k) \propto \cos (2 \pi k)$ are the eigenfunctions of ker $\hat{\mathcal{L}}$ considered in Sec. 3.2.1. They are connected to particle number and energy conservation. Hence, their eigenvalues are always zero. The eigenvalues of the other eigenfunctions are not expected to be zero for broken integrability. In Sec. 3.6.4, we will see that they increase with $\varepsilon^{2}$. App. C.9 displays how to simplify $\mathcal{L}_{m n}^{0}$ even more and how the zeros of the factor $\left|c_{p}-c_{q}\right|$ in the denominator can be eliminated analytically.

\subsubsection{First-order perturbation theory}

We have shown, that $\hat{\mathcal{L}}^{\varepsilon=0}$ has an infinitely large kernel dimension. The two basis functions $\zeta_{0}=$ const and $\zeta_{-1}=\omega^{\varepsilon=0}$ will still be eigenfunctions of $\hat{\mathcal{L}}$, even for non-zero $\varepsilon$. They are the constant function $\zeta_{0}^{\varepsilon}=$ const and the dispersion relation $\zeta_{-1}^{\varepsilon}=\omega$, respectively.

The other functions $\zeta_{n \leqslant 2}$ are eigenfunctions of $\hat{\mathcal{L}}$ in zeroth-order perturbation theory. However, they are not the $\varepsilon \rightarrow 0$ limit of the eigenfunctions $\chi^{(n)}(k)$. These $\varepsilon \rightarrow 0$ limits can be found by higher-order contributions of perturbation theory as superpositions of $\zeta_{n \leqslant 2}$. A hint that $\zeta_{n \leqslant 2}$ cannot be the equal to the eigenfunctions $\lim _{\varepsilon \rightarrow 0} \chi^{(n)}(k)$ is that all the eigenfunctions are orthogonal for $\varepsilon \neq 0$, but the functions $\zeta_{n}$ are not: $\left\langle\chi^{(m)}, \chi^{(n)}\right\rangle_{\mathrm{F}}=\delta_{m n}$ (cf. Eqs. (3.14) and (B.71)), but $\left\langle\zeta_{m}, \zeta_{n}\right\rangle_{\mathrm{F}} \neq 0$ for all $m \neq n, m, n<0$, and $\beta \neq 0^{1}$.

In Sec. 3.4, the low-lying eigenvalues were defined as those eigenvalues, which vanish in the limit $\varepsilon \rightarrow 0$. This means that they are the smallest eigenvalues. Now, we additionally introduce another designation: We refer to the eigenfunctions of low-lying eigenvalues as low-lying eigenfunctions.

\footnotetext{
${ }^{1}$ For $\beta=0$ the Fermi-Dirac function becomes $f(k)=$ const. Therefore, the scalar product $\langle\cdot, \cdot\rangle_{\mathrm{F}}$ defined in Eq. (3.14) is proportional to the standard scalar product. This makes the basis $\zeta_{n}$ orthonormal.
} 

next-to-nearest-neighbor hopping

It is very probable that the low-lying eigenvalues cannot be calculated analytically, but we will show, that they are $\mathcal{O}\left(\varepsilon^{2}\right)$. This will be done by showing that the first-order contribution $\tilde{\lambda}_{1}$ of a low-lying eigenvalue $\lambda^{\varepsilon}$ vanishes, i.e.

$$
\tilde{\lambda}_{1}=\left.\frac{d}{d \varepsilon} \lambda^{\varepsilon}\right|_{\varepsilon=0}
$$

In App. C.10, we find that $\tilde{\lambda}_{1}$ is defined by the eigenvalue problem

$$
\sum_{n=-\infty}^{-1} \mathcal{L}_{m n}^{(1)} A_{n}=\tilde{\lambda}_{1} A_{m}, \quad m \leqslant-1 .
$$

In order to solve this eigenvalue problem, we have to diagonalize the matrix $\mathcal{L}_{m n}^{(1)}$ given in Eq. (C.111):

$$
\mathcal{L}_{m n}^{(1)} \equiv\left\langle v_{m}, \hat{\mathcal{L}}_{1}\left[v_{n}\right]\right\rangle_{\mathrm{F}} \equiv\left\langle v_{m}\left|\left[\frac{d}{d \varepsilon} \hat{\mathcal{L}}\right]_{\varepsilon=0}\right| v_{n}\right\rangle=\left[\frac{d}{d \varepsilon} \mathcal{L}_{m n}^{\varepsilon}\right]_{\varepsilon=0}
$$

The next step is plugging in the matrix $\mathcal{L}_{m n}^{\varepsilon}$ given in Eq. (3.157):

$$
\begin{aligned}
& \mathcal{L}_{m n}^{(1)}=\frac{-U^{2} \Delta_{m+n}}{\hbar J} \int d p d q\left\{\frac{d}{d \varepsilon} \frac{\gamma\left(\varepsilon r_{p q}\right) \zeta_{m}\left(\tilde{k}_{p q}^{\varepsilon}+p\right)}{\left|c_{p}-c_{q}\right|}\left[1-f\left(\tilde{k}_{p q}^{\varepsilon}+p\right)\right]\left[1-f\left(\tilde{k}_{p q}^{\varepsilon}-p\right)\right]\right. \\
& \left.\quad \times f\left(\tilde{k}_{p q}^{\varepsilon}+q\right) f\left(\tilde{k}_{p q}^{\varepsilon}-q\right)\left[v_{n}\left(\tilde{k}_{p q}^{\varepsilon}+p\right)+v_{n}\left(\tilde{k}_{p q}^{\varepsilon}-p\right)-v_{n}\left(\tilde{k}_{p q}^{\varepsilon}+q\right)-v_{n}\left(\tilde{k}_{p q}^{\varepsilon}-q\right)\right]\right\}_{\varepsilon=0}
\end{aligned}
$$

for $m, n \leqslant 0$. Actually, we do not need $m=0$ or $n=0$, because we already know that the constant function is always an eigenfunction of $\hat{\mathcal{L}}$. The other eigenfunctions are orthogonal to it. The calculation in App. C.11 leads to

$$
\begin{aligned}
\mathcal{L}_{m n}^{(1)}= & \frac{U^{2} \beta}{\hbar J} \vartheta_{m} \vartheta_{n} \Delta_{m+n} \int_{\left[-\frac{1}{2}, \frac{1}{2}\right]^{2}} d p d q \frac{1+2 c_{p} c_{q}}{\left|c_{p}-c_{q}\right|}\left(c_{m p}-c_{m q}\right)\left(c_{n p}-c_{n q}\right) \\
& \times f^{0}\left(\frac{1}{4}+p\right) f^{0}\left(\frac{1}{4}-p\right) f^{0}\left(\frac{1}{4}+q\right) f^{0}\left(\frac{1}{4}-q\right)[\eta(p)+\eta(q)] .
\end{aligned}
$$

Due to the factor $\vartheta_{n}\left(c_{n p}-c_{n q}\right)$, the matrix $\mathcal{L}_{m n}^{(1)}$ vanishes for $n \leqslant 0$ for all $m$. The factor also vanishes for $m \leqslant 0$ for all $n$. This symmetry comes from the fact that $\hat{\mathcal{L}}$ is hermitian in the scalar product $\langle\cdot, \cdot\rangle_{\mathrm{F}}$.

$$
\mathcal{L}_{m, n}^{(1)}=0 \text { for } n \leqslant 0(\text { or } m \leqslant 0) .
$$

Thus, the 1.h.s. in Eq. (3.165) is zero and so is the r.h.s. $\tilde{\lambda}_{1}$. We have proven that the first-order correction to the low-lying eigenvalues of $\hat{\mathcal{L}}$ vanishes. This confirms the $\varepsilon^{2}$ behavior, we have found in the numerical calculation for the relaxation rates $\lambda_{n}$ in Fig. 3.5.

Due to the vanishing first-order contribution of the perturbation theory, the $\varepsilon \rightarrow 0$ limits of the low-lying eigenfunctions are determined by the second-order contribution of perturbation theory. However, it is important to note that in general the high-lying eigenvalues, which we define as non-low-lying eigenvalues, do have a first-order correction in $\varepsilon$. 


\subsection{Summary}

The final temperature $T$ measures the energy content of the electronic system we are studying. The more energy we have, the more excited electrons we have compared to the ground state $(T=0)$. When the energy content approaches half the possible total energy $(T \rightarrow \infty)$, the relaxation time decreases. The trend of decreasing relaxation times for increasing temperature is also observed in higher-dimensional systems, see e.g. Mueller and Rethfeld (2013). However, for small temperatures we found three scenarios:

1. Away from half filling: The relaxation rates become exponentially small as function of inverse final temperature $\beta$.

2. Half filling and $\left\langle\hat{I}_{3}\right\rangle \neq 0$ : The thermalization rate is again exponentially small as a function of $\beta$ for low temperature.

3. Half filling and $\left\langle\hat{I}_{3}\right\rangle=0$ : The thermalization rate is hardly dependent on $\beta$ and the prefactor $\tilde{\lambda}_{4}$ is of order $\mathcal{O}(1)$.

At half filling, we have to consider the operator $\hat{I}_{3} \approx \hat{K}-\frac{1}{4}\left(\hat{N}_{\mathrm{R}}-\hat{N}_{\mathrm{L}}\right)$. If it has a zero expectation value, then the eigenfunction $\chi^{(3)}(k)$ does not contribute to the perturbation $\phi(k)$ of the quasiparticle momentum distribution. This means that the time is not set by $\lambda_{3}$, which is exponentially small in $\beta$, but by $\lambda_{4}=\tilde{\lambda}_{4} \varepsilon^{2} u^{2} / t_{0}$, see Fig. 3.6. Hence, the relaxation time is much faster than in the other two cases. This is explained by Umklapp processes: For low $T$, they are only effective at half filling. 


\section{Chapter 4}

\section{Praseodymium calcium manganite}

\subsection{Introduction}

In this chapter, we apply our numerical method to a model with a more complex band structure. The substance we are interested in belongs to the class that is known as "manganites" (Dagotto, 2003), although it incorporates $\mathrm{Mn}^{3+}$ as well as $\mathrm{Mn}^{4+}$ (the latter is a manganate). In general, the manganites we are referring to have the chemical formula $\mathrm{A}_{\mathrm{x}} \mathrm{B}_{1-\mathrm{x}} \mathrm{MnO}_{3}$, where $A$ and $B$ are for example Lanthanum (La), Calcium (Ca), or Praseodymium (Pr). The manganese and the oxygen form lattices of octahedra, see Fig. 4.1. The atoms A and $\mathrm{B}$ are distributed into the remaining spaces. By placing different sorts of atoms $\mathrm{A}$ and $\mathrm{B}$ in different relative concentrations $x$, the electronic doping can be changed. For example, for $\operatorname{Pr}_{0.5} \mathrm{Ca}_{0.5} \mathrm{MnO}_{3}$, half of the manganese atoms miss an additional electron, therefore formally half of them have the oxidation state $3+$ and the other one $4+$.

Manganites are known for many fascinating features (Dagotto, 2003), with the colossal magneto resistance being the most famous one. The most important for us, however, is that it is a promising material for a more efficient conversion of solar energy to electric energy compared to the conventional silicon-based solar cells (Saucke et al., 2012), because the long lifetime of photo-excitations of electrons. Furthermore, we will show below that the manganites exhibit many different magnetic phases. These influence the band structure. Thus, one expects a tunability of the photovoltaic properties of manganites using electric and magnetic fields (Ni et al., 2012).

For understanding the conduction properties of manganites, it is essential to describe the corresponding electron orbitals. Since only the manganese and oxygen atoms contribute to electronic conduction, we investigate the change of the valence orbitals, when bringing both of them in close proximity. Thus, the first section of this chapter, Sec. 4.1.1, introduces the crystal-field splitting, which leads to the reorganization of the manganese $d$-orbitals into $e_{g}$-and $t_{2 g}$ orbitals. 


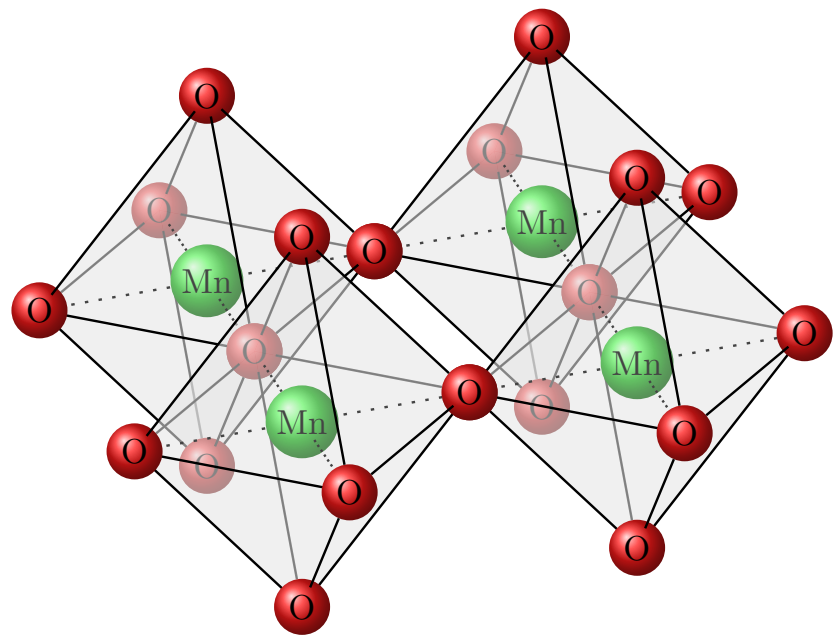

Figure 4.1: The perovskite structure of $\mathrm{A}_{1-\mathrm{x}} \mathrm{B}_{\mathrm{x}} \mathrm{MnO}_{3}$ (Dagotto, 2003). We only show the manganese and oxygen atoms. Between the octahedra, there is space for electron donors and acceptors, which are denoted by A and B. Note that the size of the ions are not to scale for a better illustration of the geometry.

In Sec. 4.1.2, we will introduce the exchange mechanisms of $\mathrm{Pr}_{\mathrm{x}} \mathrm{Ca}_{1-\mathrm{x}} \mathrm{MnO}_{3}$ (PCMO) for $x=0.5$, which leads to conduction, but also to the many possible magnetic phases in general.

\subsubsection{Crystal field splitting}

In this section, we review the splitting of the $d$ states of the manganese atoms into $\mathrm{e}_{\mathrm{g}}$ and $\mathrm{t}_{2 \mathrm{~g}}$ states. This is called crystal field splitting. We follow the derivation of Dagotto (2003).

The electronic configuration of manganese is ${ }^{1}[\operatorname{Ar}] 3 \mathrm{~d}^{5} 4 \mathrm{~s}^{2}$. The $3 \mathrm{~d}$ subshell is partly filled. Its states, which we from now on denote as $d$ states, have the highest energy compared to the states, in which the other electrons are residing. Hence, we can safely assume that the other shells are not influenced by nearby atoms. $3 \mathrm{~d}^{5}$ means that the principal quantum number of the shell is $n=3$, the angular momentum of the subshell is $l=2$, and the number of electrons in this subshell is equal to 5 . The d states change when there are oxygen atoms in the vicinity. In zeroth order of perturbation theory, the new states are a superposition of the original d states, which we will demonstrate in this section. However, it is important that in higher orders they also acquire smallf contributions from other states with $l \neq 2$

The states in the manganese $\mathrm{d}$ shell have the principle quantum number $n=3$ and the azimuthal quantum number $l=2$. Both can be considered fixed for the derivation below. The $d$ states only differ in the magnetic quantum number

\footnotetext{
${ }^{1}$ The electronic configuration of Argon (Ar) is $1 \mathrm{~s}^{2} 2 \mathrm{~s}^{2} 2 \mathrm{p}^{6} 3 \mathrm{~s}^{2} 3 \mathrm{p}^{6}$. The information about electronic configurations can be deducted from the chemical periodic table.
} 
$m$. Furthermore, they have the same eigenenergy, as long as we neglect the influence of magnetic fields on the states with different quantum number $m$. This eigenenergy we denote as $W_{n=3, l=2}$ and the unperturbed Hamiltonian of the manganese electrons as $\hat{H}_{\mathrm{Mn}}$. Hence, we have

$$
\hat{H}_{\mathrm{Mn}}|n, l, m\rangle=W_{n, l}|n, l, m\rangle .
$$

The eigenstates $|n, l, m\rangle$ in real space are given by $\langle\vec{r} \mid n, l, m\rangle=R_{n l} Y_{l m}(\theta, \phi)$ with the radial part $R_{n l}(r)$ and the spherical harmonics $Y_{l m}(\theta, \phi)$. In the following, we will not explicitly write the principal quantum $n$ any more, since we set it constant anyway.

The Hamiltonian $\hat{H}_{\mathrm{Mn}}$ for the electrons of a manganese atom is perturbed by the six adjacent oxygen atoms. The oxygen imposes a perturbative potential $\hat{V}$. The original eigenstates of $\hat{H}_{\mathrm{Mn}}$ are the $|l, m\rangle$. The perturbation $\hat{V}$ leads to new eigenstates $|\psi\rangle=\left|\psi_{0}\right\rangle+\alpha\left|\psi_{1}\right\rangle+\mathcal{O}\left(\alpha^{2}\right)$, with $\alpha$ being the small real number, to which $\hat{V}$ is proportional. Hence, we define $\hat{V}=\alpha \hat{V}^{(1)}$. As a start, we expand $|\psi\rangle=\sum_{l m} Q_{l m}|l, m\rangle$, with $Q_{l m}=Q_{l m}^{(0)}+\alpha Q_{l m}^{(1)}+\mathcal{O}\left(\alpha^{2}\right)$. The perturbation theory begins by considering the eigenvalue equation for the total Hamiltonian:

$$
\hat{H}_{\mathrm{Mn}}|\psi\rangle+\hat{V}|\psi\rangle=E|\psi\rangle
$$

where $E=E_{0}+\alpha E_{1}+\mathcal{O}\left(\alpha^{2}\right)$. The zeroth order gives

$$
\sum_{l^{\prime} m^{\prime}}\left(W_{l^{\prime}}-E_{0}\right) Q_{l^{\prime} m^{\prime}}^{(0)}\left|l^{\prime}, m^{\prime}\right\rangle=0
$$

In order to find non-zero coefficients $Q_{l^{\prime} m^{\prime}}^{(0)}$, the leading contribution $E_{0}$ of the eigenenergy must be equal to one of the coefficients $W_{l}$. This fixes the angular momentum $l$ for $|\psi\rangle$ (in our case $l=2$ ). The rest of the sum in Eq. (4.3) leads to $Q_{l^{\prime} m^{\prime}}^{(0)}=0$ for $l^{\prime} \neq l$. Thus, we have

$$
|\psi\rangle=\sum_{m^{\prime}} Q_{l, m^{\prime}}^{(0)}\left|l, m^{\prime}\right\rangle .
$$

The first order of Eq. (4.2) is

$$
\hat{H}_{\mathrm{Mn}}\left|\psi_{1}\right\rangle+\hat{V}^{(1)}\left|\psi_{0}\right\rangle=E_{1}\left|\psi_{0}\right\rangle+E_{0}\left|\psi_{1}\right\rangle
$$

We multiply $\left\langle l^{\prime}, m^{\prime}\right|$ from the left and get

$$
\begin{aligned}
0 & =\sum_{l^{\prime \prime} m^{\prime \prime}}\left\langle l^{\prime}, m^{\prime}\left|\left(\hat{H}_{\mathrm{Mn}}-E_{0}\right) Q_{l^{\prime \prime} m^{\prime \prime}}^{(1)}\right| l^{\prime \prime}, m^{\prime \prime}\right\rangle+\sum_{m^{\prime \prime}}\left\langle l^{\prime}, m^{\prime}\left|\left(\hat{V}^{(1)}-E_{1}\right) Q_{l m^{\prime \prime}}^{(0)}\right| l, m^{\prime \prime}\right\rangle \\
& =\sum_{l^{\prime \prime} m^{\prime \prime}} \delta_{l^{\prime} l^{\prime \prime}} \delta_{m^{\prime} m^{\prime \prime}}\left(W_{l^{\prime \prime}}-W_{l}\right) Q_{l^{\prime \prime} m^{\prime \prime}}^{(1)}+\sum_{m^{\prime \prime}} Q_{l m^{\prime \prime}}^{(0)}\left\langle l^{\prime}, m^{\prime}\left|\hat{V}^{(1)}\right| l, m^{\prime \prime}\right\rangle-E_{1} \delta_{l l^{\prime}} Q_{l m^{\prime}}^{(0)} \\
& =\left(W_{l^{\prime}}-W_{l}\right) Q_{l^{\prime} m^{\prime}}^{(1)}+\sum_{m^{\prime \prime}} Q_{l m^{\prime \prime}}^{(0)}\left\langle l^{\prime}, m^{\prime}\left|\hat{V}^{(1)}\right| l, m^{\prime \prime}\right\rangle-E_{1} \delta_{l l^{\prime}} Q_{l m^{\prime}}^{(0)} .
\end{aligned}
$$

For $l^{\prime}=l$, this equation yields the eigenvalue equation

$$
\sum_{m^{\prime}}\left[\left\langle l, m\left|\hat{V}^{(1)}\right| l, m^{\prime}\right\rangle-E_{1}\right] Q_{l m^{\prime}}^{(0)}=0 .
$$


The solution of this equation leads to the first-order correction $E_{1}$ of the eigenenergy $E$ as well as the leading order $Q_{l m}^{(0)}$ of the eigenstate coefficients $Q_{l m}$.

The entries of the 5-by-5 matrix $\left(\left\langle l, m\left|\hat{V}^{(1)}\right| l, m^{\prime}\right\rangle\right)_{m m^{\prime}}$ are defined by

$$
\left\langle l, m|\hat{V}| l, m^{\prime}\right\rangle=\int d^{3} r\left|R_{n l}(r)\right|^{2} Y_{l m}^{*}(\theta, \phi) V(\vec{r}) r^{l} Y_{l m^{\prime}}(\theta, \phi) .
$$

For the evaluation of the matrix elements $\left\langle l, m|\hat{V}| l, m^{\prime}\right\rangle$, we write the perturbing potential in real space. It is the superposition of the potential created by the six neighboring, negatively-charged oxygen ions:

$$
V(\vec{r})=V_{x}(\vec{r})+V_{y}(\vec{r})+V_{z}(\vec{r})
$$

with

$$
V_{s}(\vec{r})=\frac{Z e}{\sqrt{r^{2}+a^{2}-2 a s}}+\frac{Z e}{\sqrt{r^{2}+a^{2}+2 a s}} \quad \forall s \in x, y, z .
$$

Here, we defined the distance $a$ between one manganese and one oxygen, the elementary charge $e$, the effective number of charges of the oxygen ion $Z=-2$, and the Cartesian coordinates $(x, y, z)$ of position $\vec{r}$. The denominators in the expression of $V_{s}$ come from the distance measure $\left|\vec{r}-a \vec{e}_{s}\right|=\sqrt{r^{2}+a^{2} \pm 2 a s}$ with the unit vector $\vec{e}_{s}$ in $s$-direction.

We only need to know the potential close to the manganese atom. Hence, we apply a Taylor expansion, which simplifies the potential to

$$
V(\vec{r})=Z e\left[\frac{6}{a}+\frac{35}{4 a^{5}}\left(x^{4}+y^{4}+z^{4}-\frac{3}{5} r^{4}\right)\right] .
$$

Since the $\mathrm{d}$ states are described by $Y_{l m}(\theta, \phi)$, it is reasonable to rewrite the potential using spherical harmonics:

$$
V(\vec{r})=\frac{Z e}{a}\left[6+\frac{\sqrt{\pi} r^{4}}{6 a^{4}}\left(\sqrt{70}\left(Y_{4,4}+Y_{4,-4}\right)+14 Y_{4,0}\right)\right] .
$$

Since the spherical harmonics are a basis for functions dependent on the angles $\theta$ and $\phi$, we can write a product of two spherical harmonics with the same angles as a sum over single spherical harmonics. This leads to the following expression (Edmonds, 1957):

$$
\begin{aligned}
& Y_{l_{1} m_{1}}(\theta, \phi) Y_{l_{2} m_{2}}(\theta, \phi)=\sum_{l m} \sqrt{\frac{\left(2 l_{1}+1\right)\left(2 l_{2}+1\right)(2 l+1)}{4 \pi}} \\
& \quad \times\left\langle l_{1}, l_{2}, m_{1}, m_{2} \mid l_{1}, l_{2}, l, m\right\rangle\left\langle l_{1}, l_{2}, 0,0 \mid l_{1}, l_{2}, l, 0\right\rangle Y_{l m}(\theta, \phi) .
\end{aligned}
$$

In the next step, we use the following property of the Clebsch-Gordon-coefficients (Mathur and Singh, 2009):

$$
\left\langle l_{1}, l_{2}, m_{1}, m_{2} \mid l_{1}, l_{2}, l, m\right\rangle=0 \quad \text { if } m \neq m_{1}+m_{2} .
$$

This leads to

$$
\begin{aligned}
& Y_{l_{1} m_{1}}(\theta, \phi) Y_{l_{2} m_{2}}(\theta, \phi)=\sum_{l m} \sqrt{\frac{\left(2 l_{1}+1\right)\left(2 l_{2}+1\right)(2 l+1)}{4 \pi}} \\
& \quad \times\left\langle l_{1}, l_{2}, m_{1}, m_{2} \mid l_{1}, l_{2}, l, m_{1}+m_{2}\right\rangle\left\langle l_{1}, l_{2}, 0,0 \mid l_{1}, l_{2}, l, 0\right\rangle Y_{l, m_{1}+m_{2}}(\theta, \phi) .
\end{aligned}
$$




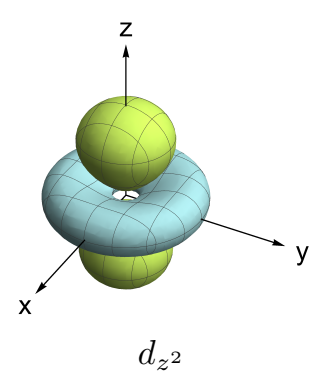

$$
R_{32}(r) Y_{2,0}(\theta, \phi)
$$

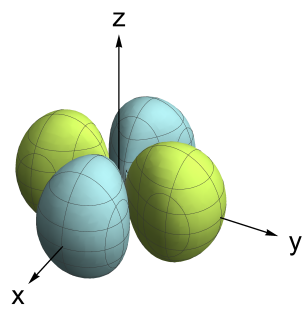

$$
d_{x^{2}-y^{2}}
$$

$R_{32}(r)\left[Y_{2,2}(\theta, \phi)+Y_{2,-2}(\theta, \phi)\right]$

eg states
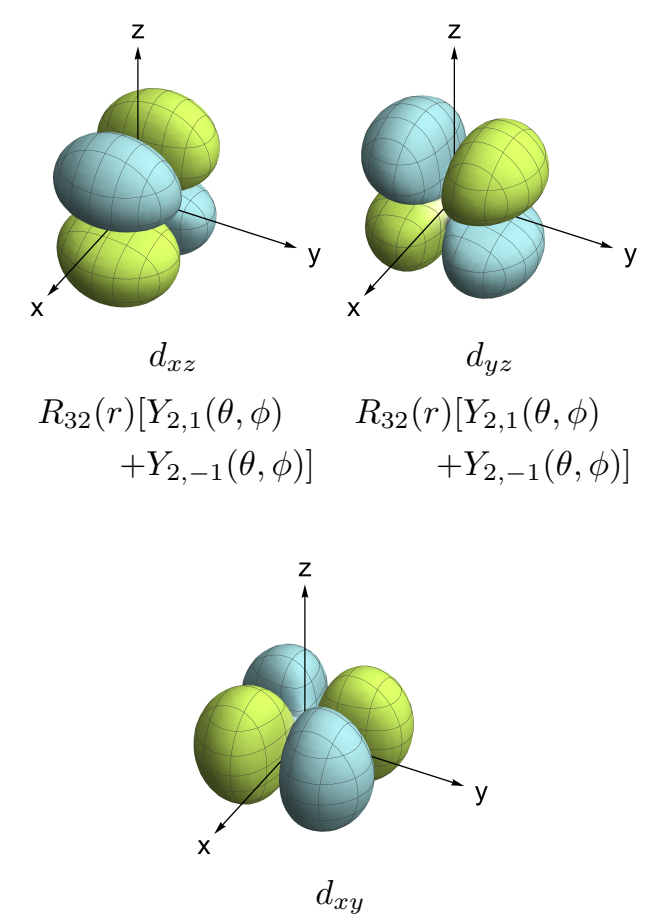

$R_{32}(r)\left[Y_{2,2}(\theta, \phi)-Y_{2,-2}(\theta, \phi)\right]$

$\mathrm{t}_{2 \mathrm{~g}}$ states

Figure 4.2: Illustration of $e_{g}$ and $t_{2 g}$ orbitals. The colors denote different signs of the wavefunction. The designation of the orbital is written below the respective plots. Below them is the formula of the highest-order contribution to the corresponding wavefunction. The $\mathrm{e}_{\mathrm{g}}$ states have a high density in the directions of $x, y$, and $z$, where the adjacent oxygen atoms are located. The $t_{2 \mathrm{~g}}$ states evade them, and thus they have a lower energy because of a smaller Coulomb repulsion with the oxygen electrons. The three-dimensional plots were created using Mathematica. 
Looking up the Clebsch-Gordon coefficients and using Eq. (4.15), Dagotto (2003) arrives at

$$
\left\langle l=2, m|\hat{V}| l=2, m^{\prime}\right\rangle=\frac{Z q C}{105}\left[\begin{array}{ccccc}
1 & 0 & 0 & 0 & 5 \\
0 & -4 & 0 & 0 & 0 \\
0 & 0 & 6 & 0 & 0 \\
0 & 0 & 0 & -4 & 0 \\
5 & 0 & 0 & 0 & 1
\end{array}\right]_{m m^{\prime}},
$$

with $C=\int d r r^{6}\left|R_{3,2}(r)\right|^{2}$. Obviously, one of the eigenvalues is 6 and there is a two-fold degenerate space with eigenvalue -4 . The remaining block matrix

$$
\left[\begin{array}{ll}
1 & 5 \\
5 & 1
\end{array}\right]
$$

has the eigenvalues $1 \pm 5 \in-4,6$. In summary, the eigenvalue 6 is two-fold and the eigenvalue -4 three-fold degenerate. The two-fold-degenerate eigenstates are denoted by $\mathrm{e}_{\mathrm{g}}$ and have a higher energy than the three-fold-degenerate ones, which are denoted by $t_{2 g}$. All five states are visualized in Fig. 4.2, which shows that the $e_{\mathrm{g}}$ states expand into the space in the direction to the oxygen atoms. Therefore the $e_{g}$ states have a larger overlap with the oxygen states than the $t_{2 g}$ states. Hence, the energy elevation of the $\mathrm{e}_{\mathrm{g}}$ states is due to the higher Coulomb repulsion with the oxygen electrons.

\subsubsection{Competing couplings}

In this section, we explain the various couplings of two neighboring manganese atoms. They are the couplings of the $e_{g}$ and $t_{2 g}$ states of neighboring manganese atoms, which are established by the valence orbitals of the oxygen, i.e. the $p$ orbitals. We will show that the $\mathrm{e}_{\mathrm{g}}$ states of the manganese form $\sigma$ bonds with the oxygen $p_{z}$ orbital. Hence, the latter is referred to as the oxygen's $\mathrm{p}_{\sigma}$ orbital. The $t_{2 \mathrm{~g}}$ states form $\pi$ bonds with the remaining $p$ orbitals, $p_{x}$ and $p_{y}$. These two oxygen orbitals are the component parts of the $\mathrm{p}_{\pi}$ orbital of the oxygen. Since manganese and oxygen have different kinds of valence orbitals, we will from now on only use the designations $\mathrm{e}_{\mathrm{g}}, \mathrm{t}_{2 \mathrm{~g}}, \mathrm{p}_{\sigma}$, and $\mathrm{p}_{\pi}$ without mentioning the sort of nuclei they belong to. In the following, we will discuss the three most important types of couplings caused by these valence orbitals.

Hund's coupling: We start with the coupling within the Mn, which is the electronic interaction of its $t_{2 g}$ and its $e_{g}$ states. The $t_{2 g}$ states contain three electrons, which have the same spin due to energy minimization (Hund's rule). The latter also leads to the e state with the same spin being lowered in energy, such that the remaining $e_{g}$ and $t_{2 g}$ states are both higher in energy and not occupied. The electrons in the $e_{g}$ and $t_{2 g}$ interact via electronic exchange, which is called Hund's coupling, and we denote it by $J_{\mathrm{H}}$.

Superexchange: The next coupling is between oxygens and manganese valence orbitals. Therefore, we consider one $\mathrm{Mn}^{4+}-\mathrm{O}^{2-}-\mathrm{Mn}^{3+}$ bridge in $z$-direction. The $\mathrm{e}_{\mathrm{g}}$ orbitals form a $\sigma$ bond with the $\mathrm{p}_{\sigma}$ orbital (i.e. the $\mathrm{p}_{\mathrm{z}}$ ) of the oxygen. 

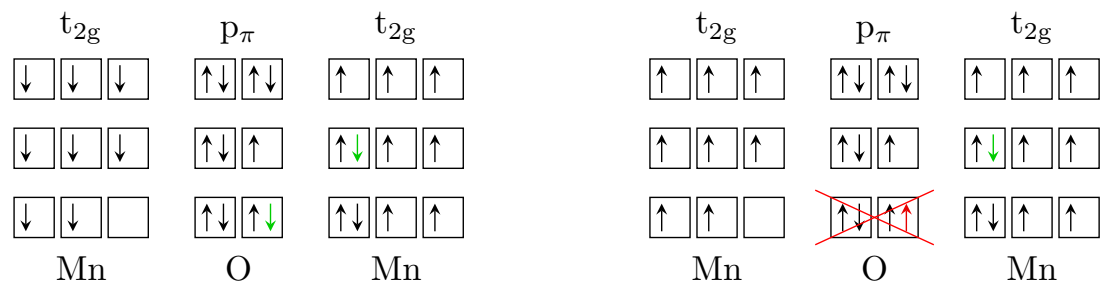

Figure 4.3: Visualization of the superexchange inducing anti-ferromagnetism (see also Li (2008), Fig. 2.10): The left picture shows the possible states of the $\pi$ bond of the fully filled oxygen- $\mathrm{p}_{\pi}$ orbitals and the half-filled $\mathrm{t}_{2 \mathrm{~g}}$ orbitals of the neighboring manganese atoms. In the left part, all the electrons of one $t_{2 g}$ have spin up, and the ones of the other $t_{2 g}$ have spin down. In this scenario, one electron can be virtually transported from one $\mathrm{Mn}$ to the next. On the right, all the $t_{2 g}$ electrons have the same spin. The electrons can only be virtually excited to neighboring $t_{2 g}$ states, but transport from one $t_{2 g}$ to the next is not possible. Although the lower two states are much higher in energy, they are mixed with the upper state and, thus, lower the energy of the upper state. The mechanism is similar to the one of an ionic bond, where there is a bond without electronic transport.

The $t_{2 g}$ orbitals form $\pi$ bonds with the remaining $\mathrm{p}_{\pi}$ orbitals (i.e. $\mathrm{p}_{\mathrm{x}}$ and $\mathrm{p}_{\mathrm{y}}$ ) of the oxygen. Because of their symmetry, the $e_{g}$ cannot form bonds with the $\mathrm{p}_{\pi}$ and the $\mathrm{t}_{2 \mathrm{~g}}$ cannot form bonds with $\mathrm{p}_{\sigma}$, as long as the angle of the $\mathrm{Mn}^{4+}$ $\mathrm{O}^{2-}-\mathrm{Mn}^{3+}$ bridge is $180^{\circ}$. It is important to mention that in real materials, there are distortions that can lower the total energy. Thence, the angle is never exactly $180^{\circ}$, but it is close enough, so that assuming $180^{\circ}$ should lead to a good approximation.

The $t_{2 \mathrm{~g}}$ states are half-filled, which means that they have either three spin up or three spin-down states occupied due to energy minimization of a single Mn atom. Establishing bonds to the neighboring nuclei, one can further lower the energy by mixing in higher states of possible $t_{2 g}-p_{\pi}-t_{2 g}$ configurations. This mechanism is visualized in Fig. 4.3. For the case where two neighboring $t_{2 g}$ orbitals have an antiferromagnetic alignment, the first excited state is created when one electron of the oxygen goes to one of the neighboring manganese. For obtaining the next excited state, one electron of the other manganese fills the hole of the oxygen. The last step is not possible if the $t_{2 g}$ spin of the two manganese nuclei are parallel aligned. This means that the antiferromagnetic alignment is slightly favored by neighboring $t_{2 g}$ orbitals. This effect is called superexchange (Goodenough, 1963; $\mathrm{Li}, 2008$ ). The strength of this coupling is denoted by $J_{\mathrm{AF}}$.

Double-exchange: The last coupling involves the $\sigma$ bond of $\mathrm{e}_{\mathrm{g}}$ and $\mathrm{p}_{\sigma}$. This can also show superexchange, but the leading coupling, which determines the preferred alignment of the $e_{g}$ electrons, is the double-exchange (Dagotto, 2003). It is illustrated in Fig. 4.4. This mechanism can transfer an electron of a $\mathrm{Mn}^{3+}$ to a $\mathrm{Mn}^{4+}$. Hereby, the oxygen bridge is the mediator. It is called double-exchange, 


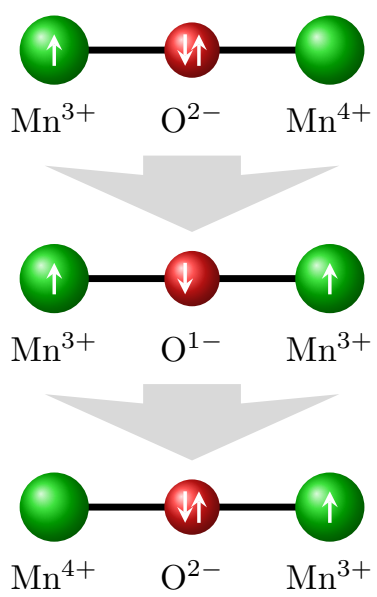

Figure 4.4: Double-exchange. Manganese atoms can exchange eg electrons by the mechanism depicted in this figure. The electron of the oxygen moves to a free $\mathrm{Mn}$; the electron of the $\mathrm{Mn}$, which was occupied in the beginning, moves to the oxygen. Due to this effect, electron transport can be effectively described by a transport from one manganese atom to another (Dagotto, 2003).

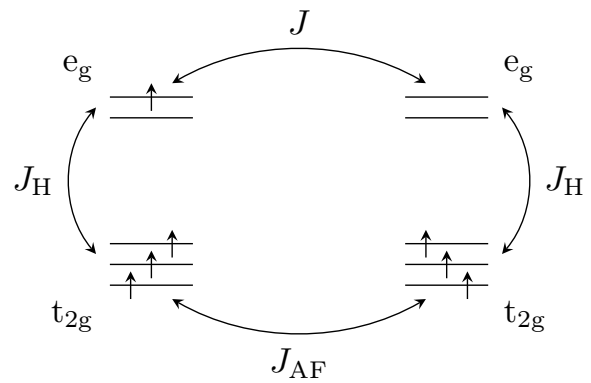

Figure 4.5: Competing couplings. We illustrate the couplings occurring in two neighboring manganese atoms, see Dagotto (2003). The $e_{g}$ states interact via the doubleexchange visualized in Fig. 4.4. It leads to the ferromagnetic hopping $J$. The ferromagnetic Hund's coupling $J_{\mathrm{H}}$ connects the $\mathrm{e}_{\mathrm{g}}$ - and $\mathrm{t}_{2 \mathrm{~g}}$ states of one atom. The neighboring $t_{2 g}$ states interact via a virtual hopping. This coupling is anti-ferromagnetic and denoted by $J_{\mathrm{AF}}$. It leads to frustration in this system of couplings, where all of the other couplings are ferromagnetic.

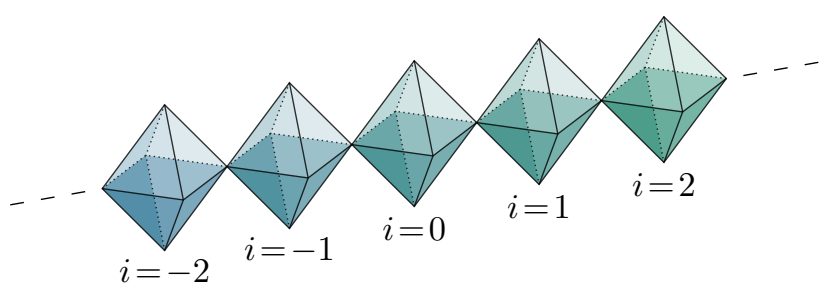

Figure 4.6: Five elements of a one-dimensional manganite chain. At the edges, oxygen atoms are located; at the center are the manganese atoms. 
because the $\mathrm{O}^{2-}$ gives the electron with the spin of the $\mathrm{Mn}^{3+}-\mathrm{e}_{\mathrm{g}}$-electron to $\mathrm{Mn}^{4+}$ and the $\mathrm{Mn}^{3+}$ gives its electron to the oxygen. This effective ferromagnetic hopping is denoted by $J$. The important difference to the superexchange of the $t_{2 g}$ electrons is that the double-exchange makes the $e_{g}$ electrons itinerant.

In this section, we introduced the couplings of neighboring manganese atoms. The Hund's coupling and the double-exchange are ferromagnetic couplings, whereas the superexchange is antiferromagnetic in nature. The difference between superexchange and double-exchange is that the former is a static coupling, whereas the latter involves itinerant electrons. The couplings $J$ and $J_{\mathrm{AF}}$ compete for the nature of the alignment of the neighboring $t_{2 g}$ spin, which can be parallel or anti-parallel. This competition is visualized in Fig. 4.5.

\subsubsection{Magnetic phases and one-dimensional chain}

Due to the competing couplings introduced in the previous section, the $t_{2 \mathrm{~g}}$ orbitals of two neighboring manganese atoms can show different alignments of their total spins. This leads to various magnetic phases depicted in Fig. 4.7, see Dagotto (2003). There can also be more than one phase in one realization of a manganite crystal. Then, phase boundaries occur.

We will consider a one-dimensional $\mathrm{Pr}_{\mathrm{x}} \mathrm{Ca}_{1-\mathrm{x}} \mathrm{MnO}_{3}$ chain, i.e. a chain of octahedra, see Fig. 4.6. Different magnetic phases also occur for different dopings. For our chain, we choose the doping $x=0.5$, where one manganese atom accommodates 3.5 electrons on average. There, the $\mathrm{e}_{\mathrm{g}}$ electrons can only move along bonds of manganese $\mathrm{d}_{\mathrm{z}^{2}}$ and the oxygen $\mathrm{p}_{\mathrm{z}}$ with a hopping amplitude $J$. Rajpurohit and Blöchl (2016) found, that for a certain hopping amplitude $J \approx 0.5 \mathrm{eV}$, which is experimentally reasonable, the total spins of the $\mathrm{t}_{2 \mathrm{~g}}$ orbitals align in an extraordinary way. They alternate between ferromagnetic and anti-ferromagnetic alignment, i.e. $\uparrow \uparrow \downarrow \downarrow \uparrow \uparrow \downarrow \downarrow$ and so on.

\subsubsection{Outline}

We introduced the most important features of manganites for this thesis while using PCMO as an example. Sec. 4.2 establishes an effective model for the manganite phase $\uparrow \uparrow \downarrow \downarrow$. Furthermore, we reshape the corresponding effective Hamiltonian to obtain the single-particle band structure. In Sec. 4.3, we adapt the multi-band Boltzmann equation to the effective model, and linearize it. Moreover, we also discuss the significance of numerous analytically zero eigenvalues of the linearized Boltzmann equation in Sec. 4.3.4. The results of this chapter are discussed in Sec. 4.4. There, we find the thermalization rates of the effective model for different temperatures and investigate $\Delta=4$.

\subsection{Effective model for the magnetic phase $\uparrow \uparrow \downarrow \downarrow$}

Our first intermediate objective is to derive an effective model for the conduction electrons, i.e. the $\mathrm{e}_{\mathrm{g}}$ electrons. Thereby, the spin configuration of the nonconducting $t_{2 \mathrm{~g}}$ orbitals, which is assumed to be static, plays an important role. 


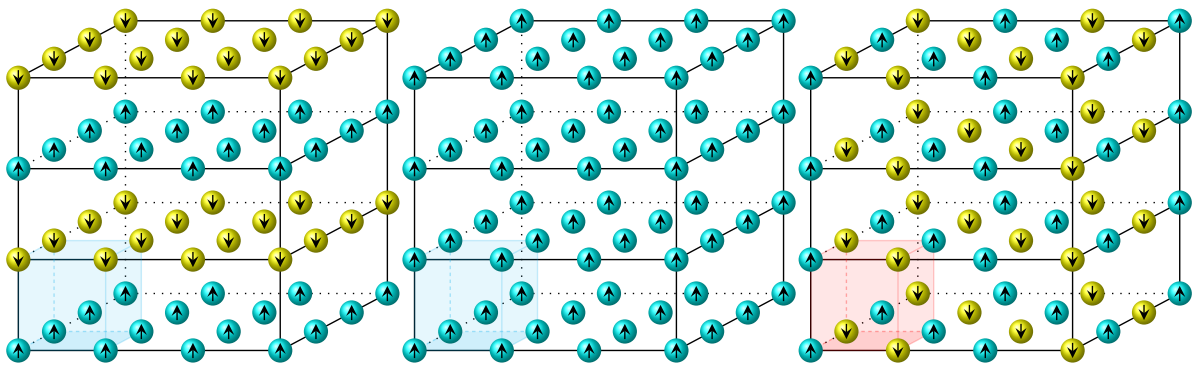

A-type

B-type (FM)

C-type

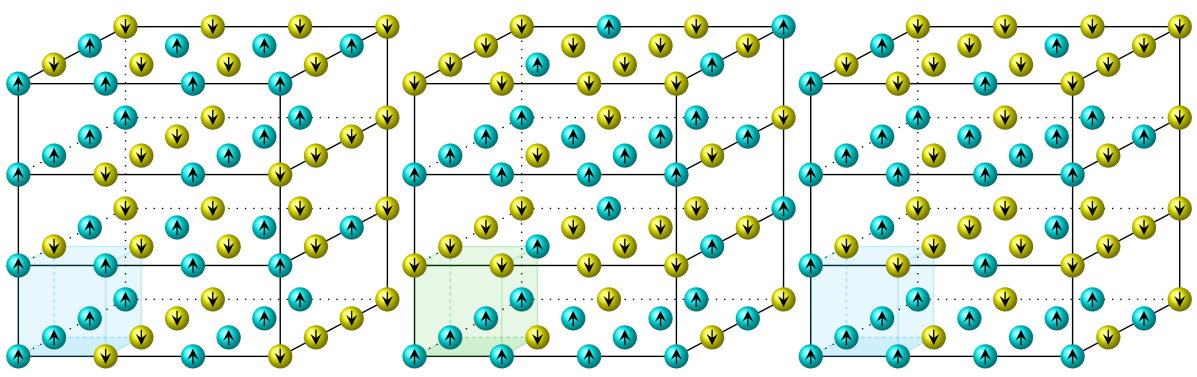

D-type

E-type

F-type

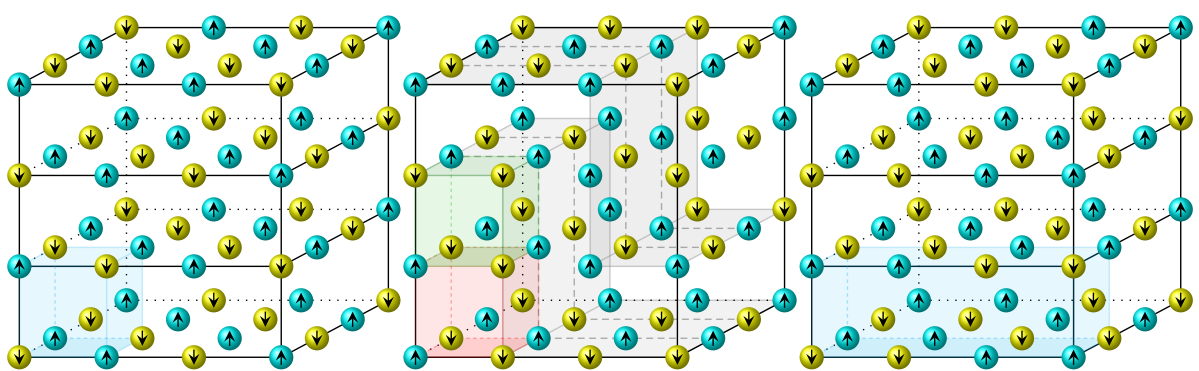

G-type

CE-type

$\mathrm{AF}(2)$

Figure 4.7: Structure types. We display the configurations found by Wollan and Koehler (1955) apart from DE-type and the DF-type. In every picture, we show the magnetization of the manganese atoms. Furthermore, one can see the AF(2)-type defined in Yunoki et al. (2000). Note that these phases also exhibit charge ordering of the manganese cores. There are several $\mathrm{Mn}^{3+}$ and $\mathrm{Mn}^{4+}$ combinations for each phase, which are presented in the work of Wollan and Koehler (1955). This shows how rich the manganite materials can be. They can exhibit ferromagnetism (FM) as well as a lot of antiferromagnetic (AF) configurations. While the B-type is completely ferromagnetic, the A-type has antiferromagnetically coupled ferromagnetic layers. The C-type follows the same principle, only the layers are diagonally aligned. In the D-type, one layer contains ferromagnetic lines, which are antiferromagnatically coupled. The next layer is rotated by $90^{\circ}$, and so on. The E-, F-, and G-type differ from the A-type by regular patterns of single opposite spins in the layers. The CE-type is a combination of the Cand the E-type resulting in a far bigger magnetic unit cell. Essentially, it is a zig-zag pattern of ferromagnetic strings. The $\operatorname{AF}(2)$ has the second biggest magnetic unit. In two directions, the coupling is antiferromagnetic, but in one direction, one sees a pattern alternating in ferromagnetism and antiferromagnetism, i.e. $\uparrow \uparrow \downarrow \downarrow \uparrow \uparrow \downarrow \downarrow \cdots$. 


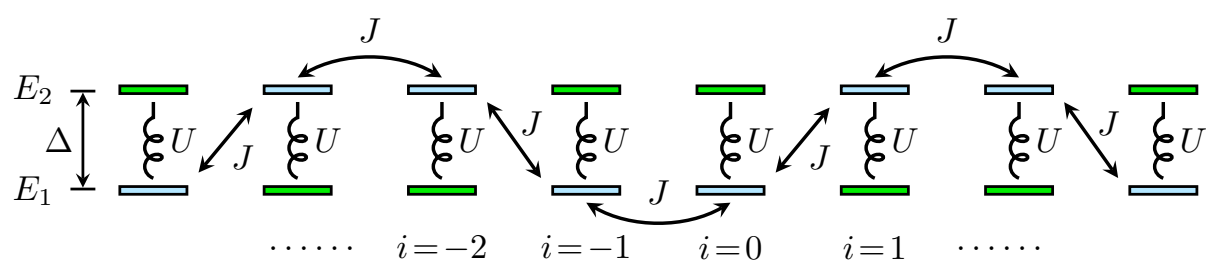

Figure 4.8: Sketch of the effective model describing the electron gas of a manganite chain in a certain magnetic phase. We are only including the $e_{g}$ states, because they are responsible for the electronic conductivity. The effect of the $t_{2 g}$ states is also accounted for. Their electrons have the same spin leading to a magnetic field, which splits the eg states by an energy $\Delta$. At every second atom, the magnetization changes, and so does the splitting of the $e_{g}$ states. The spin- $\uparrow$ states are colored in blue, the spin- $\downarrow$ states in green. The spin- $\uparrow$ electrons can hop along the blue states with a hopping amplitude $J$. They interact with the spin- $\downarrow$ electrons when they occupy the same manganese atom with the interaction strength $U$.

Their total spin splits the conducting $e_{g}$ orbital energetically. The $e_{g}$ state with spin in the direction of the total $t_{2 g}$ spin is lower in energy than the $e_{g}$ state with the spin in the other direction. As mentioned in the last section, the alignment of the $t_{2 \mathrm{~g}}$ orbitals is $\uparrow \uparrow \downarrow \uparrow \uparrow \uparrow \downarrow \downarrow$, which leads to the the effective model sketched in Fig. 4.8. Neighboring states with the same spin are connected via the hopping amplitude $J$. On each site, the $\uparrow$ state and the $\downarrow$ state have an on-site interaction $U$. The energy difference between the $\uparrow$-state energy and the $\downarrow$-state energy is $\Delta$. We measure $\Delta$ in units of $J$.

In Sec. 4.2.1, we will calculate the Hamiltonian of the effective model. Then, we will rewrite the Hamiltonian into a unit cell form, see Sec. 4.2.2. We will then transform it in Sec. 4.2.3 into a new basis where the single particle part of the Hamiltonian is diagonal. With this transformation, we map the interaction part of the Hamiltonian into a form that can be used for the Boltzmann equation, see Sec. 4.3

\subsubsection{Effective Hamiltonian}

One-particle Hamiltonian: In this section, we derive the effective Hamiltonian describing the $e_{g}$ electrons of a one-dimensional manganite chain. It is sketched in Fig. 4.8. First, we write down the quadratic part of the Hamiltonian, i.e. everything short of the on-site interaction $U$. Let the manganese sites be enumerated by $i \in \mathbb{Z}$ and the spin of the electrons denoted by $\sigma \in\{\uparrow, \downarrow\}$. For spin- $\uparrow$, the states of the sites in the set $M_{1}:=4 \mathbb{Z} \cup(4 \mathbb{Z}+3)$ have the energy $E_{1}$, the states of the sites in $M_{2}:=(4 \mathbb{Z}+1) \cup(4 \mathbb{Z}+2)$ have the energy $E_{2}$. Then, the model describing the quadratic part of the Hamiltonian depicted in Fig. 4.8 is

$$
\hat{H}_{0}=-J \sum_{\sigma i} \tilde{a}_{\sigma i}^{\dagger} \tilde{a}_{\sigma, i+1}+H . c .+\sum_{i \in M_{1}}\left[E_{1} \tilde{n}_{\uparrow i}+E_{2} \tilde{n}_{\downarrow i}\right]+\sum_{i \in M_{2}}\left[E_{2} \tilde{n}_{\uparrow i}+E_{1} \tilde{n}_{\downarrow i}\right],
$$


with the creation operator $\tilde{a}_{\sigma i}^{\dagger}$, the annihilation operator $\tilde{a}_{\sigma, i}$, and the number operator $\tilde{n}_{\sigma i}:=\tilde{a}_{\sigma i}^{\dagger} \tilde{a}_{\sigma i}$. The first two terms model the hopping of an electron from one $\mathrm{e}_{\mathrm{g}}$ to the next with a hopping amplitude $J$, which results from the previously explained double-exchange mechanism. The sums over $M_{1}$ and $M_{2}$ describe the staggered field induced by the $t_{2 g}$ spins that are assumed to be static. Moreover, note that $\tilde{a}_{\sigma i}^{\dagger}$ and $\tilde{a}_{\sigma, i}$ have a tilde. Later on, we will transform these operators into new ones, which will be marked by a hat.

In order to get one sum over all sites, we introduce the factor

$$
\zeta_{i}=\sqrt{2} \cos \left(\frac{\pi}{2} i-\frac{\pi}{4}\right)= \begin{cases}1 & \text { for } i \in M_{1} \\ -1 & \text { for } i \in M_{2}\end{cases}
$$

which distinguishes the sites in $M_{1}$ from the sites in $M_{2}$. Hence, the factor $\left(1+\zeta_{j}\right) / 2$ only selects sites in $M_{1}$ and the factor $\left(1-\zeta_{j}\right) / 2$ only sites in $M_{2}$.

$$
\begin{aligned}
& \sum_{i \in M_{1}}\left[E_{1} \tilde{n}_{\uparrow i}+E_{2} \tilde{n}_{\downarrow i}\right]+\sum_{i \in M_{2}}\left[E_{2} \tilde{n}_{\uparrow i}+E_{1} \tilde{n}_{\downarrow i}\right] \\
& =\sum_{i}\left[\left(\frac{1+\zeta_{i}}{2} E_{1}+\frac{1-\zeta_{i}}{2} E_{2}\right) \tilde{n}_{\uparrow i}+\left(\frac{1+\zeta_{i}}{2} E_{2}+\frac{1-\zeta_{i}}{2} E_{1}\right) \tilde{n}_{\downarrow i}\right] \\
& =\sum_{i}\left[\left(\frac{E_{1}+E_{2}}{2}+\zeta_{i} \frac{E_{1}-E_{2}}{2}\right) \tilde{n}_{\uparrow i}+\left(\frac{E_{2}+E_{1}}{2}+\zeta_{i} \frac{E_{2}-E_{1}}{2}\right) \tilde{n}_{\downarrow i}\right] \\
& =\sum_{\sigma i} \frac{E_{1}+E_{2}}{2} \tilde{n}_{\sigma i}+\sum_{i} \zeta_{i} \frac{E_{1}-E_{2}}{2}\left[\tilde{n}_{\uparrow i}-\tilde{n}_{\downarrow i}\right] .
\end{aligned}
$$

This leads to

$$
\hat{H}_{0}=-J \sum_{\sigma i} \tilde{a}_{\sigma i}^{\dagger} \tilde{a}_{\sigma, i+1}+H . c .+\sum_{\sigma i} \frac{E_{1}+E_{2}}{2} \tilde{n}_{\sigma i}+\sum_{i} \frac{E_{1}-E_{2}}{2} \zeta_{i}\left[\tilde{n}_{\uparrow i}-\tilde{n}_{\downarrow i}\right],
$$

where $\zeta_{i}=\sqrt{2} \cos \left(\frac{\pi}{2} i+\frac{\pi}{4}\right)$. Now, in order to give a physical meaning to the terms we derived, we define

$$
\begin{aligned}
\text { the energy difference } \Delta & :=\left(E_{2}-E_{1}\right) / J \\
\text { a spin operator } \tilde{s}_{i}^{\mathrm{Z}} & :=\frac{1}{2}\left(\tilde{n}_{\uparrow i}-\tilde{n}_{\downarrow i}\right) \\
\text { the total number operator } \tilde{N} & :=\sum_{\sigma i} \tilde{n}_{\sigma i} .
\end{aligned}
$$

Note that we defined the spin operator $\tilde{s}_{i}^{\mathrm{z}}$ without $\hbar$ for simplicity. Using all the three definitions, the Hamiltonian becomes

$$
\hat{H}_{0}=-J \sum_{\sigma i} \tilde{a}_{\sigma i}^{\dagger} \tilde{a}_{\sigma, i+1}+H . c .+\frac{E_{1}+E_{2}}{2} \tilde{N}-J \Delta \sum_{i} \zeta_{i} \tilde{s}_{i}^{\mathrm{Z}} .
$$

We see that the $\Delta$ term acts as a magnetic-field term, despite the fact the underlying mechanism is Hund's rule exchange. Now, we shift the energy levels so that the zero energy is halfway between $E_{1}$ and $E_{2}$, i.e. $E_{1}+E_{2}=0$.

$$
\hat{H}_{0}=J\left\{-\sum_{\sigma i} \tilde{a}_{\sigma i}^{\dagger} \tilde{a}_{\sigma, i+1}+H . c .-\Delta \sum_{i} \zeta_{i} \tilde{s}_{i}^{Z}\right\}
$$




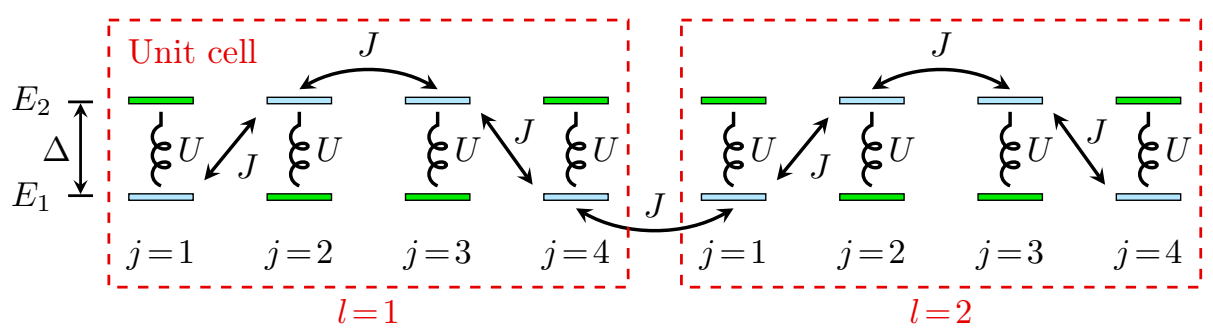

Figure 4.9: Visualization of the unit cell. Here, we show the same model depicted in Fig. 4.8. The difference is that we mark our choice of the unit cell, where the index $l$ numbers the unit cell, and the index $j$ the sites within one unit cell.

Interaction Hamiltonian: We model the interaction of the electrons by the short-ranged interaction term from the Hubbard model:

$$
\hat{H}_{\mathrm{int}}=U \sum_{i \in \mathbb{Z}} \tilde{n}_{\uparrow i} \tilde{n}_{\downarrow i}
$$

This interaction term describes the Coulomb interaction between the $e_{\mathrm{g}}$ electrons. We only take into account on-site interaction assuming that the Coulomb interaction is sufficiently screened by the positively-charged atom cores. Moreover, we define a dimensionless interaction strength $u:=U / J$.

Total Hamiltonian: In summary, the complete effective Hamiltonian is

$$
\begin{aligned}
\hat{H} & =\hat{H}_{0}+\hat{H}_{\mathrm{int}} \\
& =J\left\{-\sum_{\sigma i} \tilde{a}_{\sigma i}^{\dagger} \tilde{a}_{\sigma, i+1}+H . c .-\Delta \sum_{i} \zeta_{i} \tilde{s}_{i}^{z}+u \sum_{j} \tilde{n}_{\uparrow i} \tilde{n}_{\downarrow i}\right\} .
\end{aligned}
$$

\subsubsection{Hamiltonian in unit cell form}

In this section, we rewrite our effective Hamiltonian in such a way that the unit cell becomes apparent.

One-particle Hamiltonian: We start by considering the single-particle Hamiltonian defined in Eq. (4.18). First, we define new coordinates: $l \in \mathbb{Z}$ enumerates the unit cells and $j \in\{1,2,3,4\}$ the 4 sites in the unit cell. Therefore, the original coordinate is recovered by $i=4 l+j$. The next step is labeling the creation operator by the new coordinates, hence $\tilde{b}_{\sigma l j}^{\dagger}:=\tilde{a}_{\sigma, 4 l+j}^{\dagger}$. Thus, Eq. (4.26) becomes

$$
\hat{H}_{0}=\sum_{\sigma l j j^{\prime}}\left[\mathcal{A}_{\sigma j j^{\prime}} \tilde{b}_{\sigma l j}^{\dagger} \tilde{b}_{\sigma l j^{\prime}}+\left(\mathcal{B}_{j j^{\prime}} \tilde{b}_{\sigma l j}^{\dagger} \tilde{b}_{\sigma l+1, j^{\prime}}+H . c .\right)\right],
$$



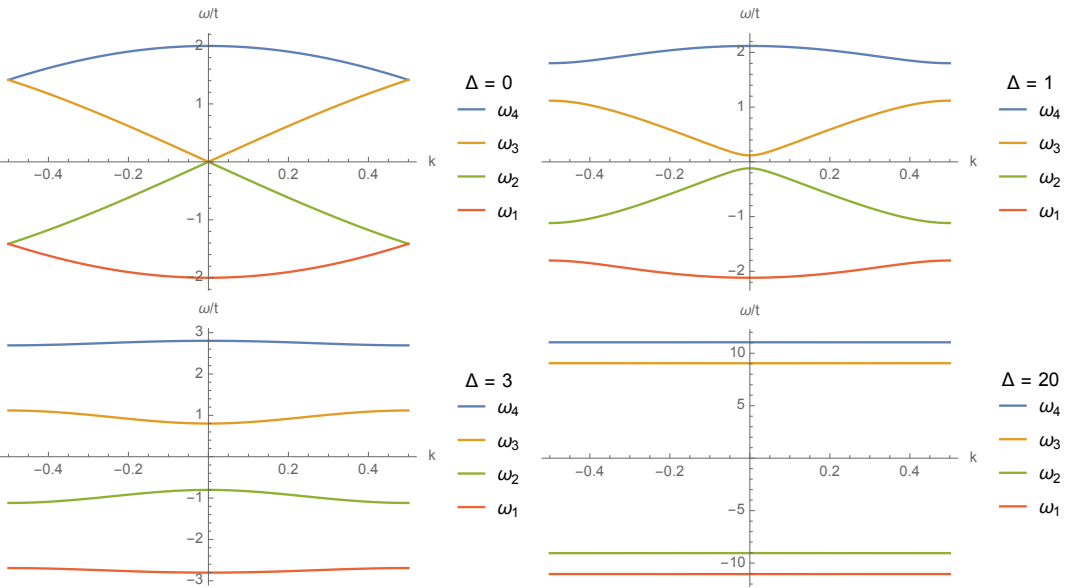

Figure 4.10: Band structure $\omega_{\nu}(k)$ for different $\Delta$ with $E_{2}=-E_{1}=J \Delta / 2$.

with the newly defined $4 \times 4$ matrices

$$
\mathcal{A}_{\uparrow}:=\left[\begin{array}{cccc}
E_{1} & -J & 0 & 0 \\
-J & E_{2} & -J & 0 \\
0 & -J & E_{2} & -J \\
0 & 0 & -J & E_{1}
\end{array}\right], \mathcal{A}_{\downarrow}:=\left[\begin{array}{cccc}
E_{2} & -J & 0 & 0 \\
-J & E_{1} & -J & 0 \\
0 & -J & E_{1} & -J \\
0 & 0 & -J & E_{2}
\end{array}\right], \mathcal{B}:=\left[\begin{array}{cccc}
0 & 0 & 0 & 0 \\
0 & 0 & 0 & 0 \\
0 & 0 & 0 & 0 \\
-J & 0 & 0 & 0
\end{array}\right] .
$$

The matrices $\mathcal{A}_{\sigma}$ describe the energy levels and the hopping within one unit cell. The hopping between two unit cells is described by $\mathcal{B}$. It is important to note that, although $\mathcal{B}$ is not hermitian, the single-particle Hamiltonian $\hat{H}_{0}$ is, because it includes the hermitian conjugate of the term containing $\mathcal{B}$.

Interaction Hamiltonian: The interaction part is brought into unit cell form simply by using the new creation operators and summing over the unit cells and the atoms they contain:

$$
\hat{H}_{\mathrm{int}}=U \sum_{l j} \tilde{b}_{\uparrow l j}^{\dagger} \tilde{b}_{\uparrow l j} \tilde{b}_{\downarrow l j}^{\dagger} \tilde{b}_{\downarrow l j} \cdot
$$

Total Hamiltonian: Combining $\hat{H}_{0}$ and $\hat{H}_{\text {int }}$, we obtain the full Hamiltonian of our effective model, which is

$$
\begin{aligned}
& \hat{H}=\hat{H}_{0}+\hat{H}_{\mathrm{int}} \\
& =\sum_{\sigma l j j^{\prime}}\left[\mathcal{A}_{\sigma j j^{\prime}} \tilde{b}_{\sigma l j}^{\dagger} \tilde{b}_{\sigma l j^{\prime}}+\mathcal{B}_{j j^{\prime}} \tilde{b}_{\sigma l j}^{\dagger} \tilde{b}_{\sigma l+1, j}+\mathcal{B}_{j j^{\prime}}^{\dagger} \tilde{b}_{\sigma l j}^{\dagger} \tilde{b}_{\sigma, l-1, j}\right] \\
& \quad+U \sum_{l j} \tilde{b}_{\uparrow l j}^{\dagger} \tilde{b}_{\uparrow l j} \tilde{b}_{\downarrow l j}^{\dagger} \tilde{b}_{\downarrow l j} .
\end{aligned}
$$




\subsubsection{Hamiltonian in single-particle band form}

Now, we bring the effective Hamiltonian finally in the form that is used to derive the multi-band Boltzmann equation, see Chapter 2. We do this by applying two transformations of the creation and annihilation operators. These two transformations are obtained by diagonalizing the single-particle Hamiltonian.

Single-particle Hamiltonian: The first transformation of the creation and annihilation operator is a Fourier transform of the unit-cell index $l$ :

$$
\tilde{b}_{\sigma l j}=\int d k e^{i 2 \pi k l} \hat{b}_{\sigma j}(k)
$$

This results into the single-particle Hamiltonian

$$
\hat{H}_{0}=\sum_{\sigma j j^{\prime}} \int d k \hat{b}_{\sigma j}^{\dagger}(k) \hat{b}_{\sigma j^{\prime}}(k) \underbrace{\left[\mathcal{A}_{\sigma j j^{\prime}}+\mathcal{B}_{j j^{\prime}} e^{i 2 \pi k}+\mathcal{B}_{j j^{\prime}}^{\dagger} e^{-i 2 \pi k}\right]}_{=: \mathcal{C}_{\sigma j j^{\prime}}(k)} .
$$

Diagonalizing the hermitian 4-by-4 matrices $\mathcal{C}_{\sigma}(k)$, we obtain the single-particle bands labeled by the indices $\nu$ :

$$
\begin{gathered}
T_{\sigma}^{\dagger}(k) \mathcal{C}_{\sigma}(k) T_{\sigma}(k)=\mathcal{D}_{\sigma}(k), \\
\mathcal{D}_{\sigma \nu \nu^{\prime}}(k)=J \delta_{\nu \nu^{\prime}} \omega_{\sigma \nu}(k),
\end{gathered}
$$

where $\omega_{\sigma \nu}(k)$ is defined in such a way that it is dimensionless. Thence, we define the final annihilation operator $\hat{c}_{\sigma \nu}(k)$ by

$$
\hat{b}_{\sigma j}(k)=\sum_{\nu} T_{\sigma j \nu}^{*}(k) \hat{c}_{\sigma \nu}(k) .
$$

Thus, the one-particle part of the Hamiltonian becomes

$$
\begin{aligned}
\hat{H}_{0} & =J \sum_{\sigma j j^{\prime} \nu} \int d k \hat{b}_{\sigma j}^{\dagger}(k) \hat{b}_{\sigma j^{\prime}}(k) T_{\sigma j \nu}(k) \omega_{\sigma \nu}(k) T_{\sigma \nu j^{\prime}}^{*}(k) \\
& =J \sum_{\sigma \nu} \int d k \omega_{\sigma \nu}(k) \hat{n}_{\sigma \nu}(k)
\end{aligned}
$$

where $\hat{n}_{\sigma \nu}(k)=\hat{c}_{\sigma \nu}^{\dagger}(k) \hat{c}_{\sigma \nu}(k)$.

The band structure of $\uparrow$ and $\downarrow$-spin particles are the same. Hence, we label both band structures the same way:

$$
\omega_{\nu}(k)=\omega_{\downarrow \nu}(k)=\omega_{\uparrow \nu}(k) .
$$

Furthermore, we order the bands by the their minimum energy. This leads to the final form of the one-particle part of the Hamiltonian:

$$
\hat{H}_{0}=J \sum_{\sigma \nu} \int d k \omega_{\nu}(k) \hat{n}_{\sigma \nu}(k)
$$


Defining $\Delta:=\left(E_{2}-E_{1}\right) / J$ and having $E_{1}+E_{2}=0$, the bands turn out to be

$$
\omega_{\nu}(k)=\stackrel{+}{ \pm} \frac{1}{2} \sqrt{8+\Delta^{2} \underset{+}{ \pm} 4 \sqrt{2(1+\cos (2 \pi k))+\Delta^{2}}} .
$$

In Fig. 4.10, these bands are plotted for various values of $\Delta$.

In the limit $\Delta=0$, we recover the single band of the tight-binding Hubbard model in the following way: The highest and the lowest bands are $\pm 2 \cos (\pi k / 2)$. The two bands in the middle are $\pm 2 \sin (\pi k / 2)$. Now, we concatenate the bands starting with the lowest. This results in the sequence

$$
\begin{aligned}
& -2 \cos (\pi k / 2), \\
& 2 \sin (\pi(k+1) / 2)=-2 \cos (\pi k / 2), \\
& 2 \cos (\pi(k+2) / 2)=-2 \cos (\pi k / 2), \text { and } \\
& 2 \sin (\pi(k+3) / 2)=-2 \cos (\pi k / 2) .
\end{aligned}
$$

Hence, for $\Delta=0$, we can describe the band structure by one band. Using the momenta $\tilde{k}=k / 4$, we obtain the cosine band of the Hubbard model $-2 \cos (2 \pi \tilde{k})$. The factor 4 resembles the change from a unit cell with four atoms to a unit cell with one atom.

For large $\Delta$, the bands become cosine shaped. This can be seen expanding for large $\Delta$ :

$$
\begin{aligned}
\omega_{\nu}(k) & =\stackrel{+}{ \pm} \frac{1}{2}\left[\Delta \underset{\mp}{ \pm} 2+\frac{2}{\Delta}+\frac{1}{\Delta^{2}}\left(4_{ \pm}^{\mp} 6\right) \stackrel{ \pm}{\mp} \frac{2}{\Delta^{2}} \cos (2 \pi k)+\mathcal{O}\left(\Delta^{-3}\right)\right] \\
& =\omega_{1,2,3,4}^{(0)} \stackrel{ \pm}{ \pm} \frac{1}{\Delta^{2}} \cos (2 \pi k)+\mathcal{O}\left(\Delta^{-3}\right),
\end{aligned}
$$

where we defined the constant $\omega_{1,2,3,4}^{(0)}:= \pm \pm+\frac{1}{2} \sqrt{8+\Delta^{2} \frac{ \pm}{\overline{+}} 4 \sqrt{2+\Delta^{2}}}$. In this case, all the band widths become $2 J / \Delta^{2}$. One obtains very flat, cosine-shaped bands. A flat band $\omega_{\nu}(k)$ means that the group velocity $\omega_{\nu}^{\prime}(k)$ is very small. Consequently, the effective hopping must be very small. For the PCMO chain, this stems from the fact that the probability for an $\uparrow$-electron to tunnel through a dimer of two elevated $\uparrow$ states becomes smaller with increasing level splitting $\Delta$. For $\Delta=\infty$, this tunneling process is not possible any more and the electrons cannot leave from their dimers. Therefore, in this limit, the effective model becomes a chain of disconnected dimers.

This has an important consequence for thermalization: For large (but finite) $\Delta$, the bands become so flat that some inter-band exchanges are not possible any more. In other words, there are exactly conserved quantities, which are certain combinations of the number operator

$$
N_{\nu}:=\int d k n_{\nu}(k),
$$

which counts the number of particles in the band $\nu$.

For two bands, such a conserved quantity occurs when their distance is larger than the maximum of their bandwidths. Numerically, one can easily find that for the lower two bands this happens at $\Delta=\Delta_{1} \approx 1.297$ and for the two bands 
in the middle at $\Delta=\Delta_{2} \approx 2.246$. Thus, already for not too large $\Delta$, certain inter-band exchanges will become impossible. Hence, there can be different chemical potentials for each band

$$
f_{\nu}(k)=\frac{1}{\exp \left\{\beta\left(\omega_{\nu}(k)-\mu_{\nu}\right)\right\}+1},
$$

and they cannot equilibrate to a common value.

Considering all four bands, the symmetry of the band structure still allows for certain inter-band exchanges. For example, a particle can be transferred from the $1^{\text {st }}$ into the $2^{\text {nd }}$ band if another particle is transferred from the $4^{\text {th }}$ into the $3^{\text {rd }}$ band. We denote this process as $(1,4 \rightarrow 2,3)$. Also, processes like $(1,2 \rightarrow 3,4)$ are energetically allowed. We will show that this results in the conserved quantities that we find after the linearization of the Boltzmann equation in Sec. 4.3.4.

Interaction Hamiltonian: Now that we have the transformation that leads to the single-particle bands, we can use them to bring the interaction Hamiltonian in the form that is necessary for setting up the Boltzmann equation. Starting from Eq. (4.29), we deduce:

$$
\begin{aligned}
& \hat{H}_{\mathrm{int}}=U \sum_{l j} \tilde{b}_{\uparrow l j}^{\dagger} \tilde{b}_{\uparrow l j} \tilde{b}_{\downarrow l j}^{\dagger} \tilde{b}_{\downarrow l j} \\
& \stackrel{(4.31)}{=} U \sum_{j} \int d^{4} k \delta\left(\Delta k_{\vec{k}}\right) \hat{b}_{\uparrow j}^{\dagger}\left(k_{1}\right) \hat{b}_{\downarrow j}^{\dagger}\left(k_{2}\right) \hat{b}_{\downarrow j}\left(k_{3}\right) \hat{b}_{\uparrow j}\left(k_{4}\right) \\
& \stackrel{(4.34)}{=} U \sum_{\vec{\nu}} \int d^{4} k \delta\left(\Delta k_{\vec{k}}\right) \Phi_{\vec{\nu}}(\vec{k}) \hat{c}_{\uparrow \nu_{1}}^{\dagger}\left(k_{1}\right) \hat{c}_{\downarrow \nu_{2}}^{\dagger}\left(k_{2}\right) \hat{c}_{\downarrow \nu_{3}}\left(k_{3}\right) \hat{c}_{\uparrow \nu_{4}}\left(k_{4}\right),
\end{aligned}
$$

with the momentum change $\Delta k_{\vec{k}}=k_{1}+k_{2}-k_{3}-k_{4}$ and the interaction weight

$$
\Phi_{\vec{\nu}}(\vec{k})=\sum_{j} T_{\uparrow j, \nu_{1}}^{*}\left(k_{1}\right) T_{\downarrow j, \nu_{2}}^{*}\left(k_{2}\right) T_{\downarrow j, \nu_{3}}\left(k_{3}\right) T_{\uparrow j, \nu_{4}}\left(k_{4}\right) .
$$

Total Hamiltonian: To summarize, we find for the effective Hamiltonian in band form:

$$
\begin{aligned}
\hat{H}= & \hat{H}_{0}+\hat{H}_{\mathrm{int}} \\
= & J \sum_{\sigma \nu} \int d k \omega_{\nu}(k) \hat{n}_{\sigma \nu}(k) \\
& \quad+U \sum_{\vec{\nu}} \int d^{4} k \delta\left(\Delta k_{\vec{k}}\right) \Phi_{\vec{\nu}}(\vec{k}) \hat{c}_{\uparrow \nu_{1}}^{\dagger}\left(k_{1}\right) \hat{c}_{\downarrow \nu_{2}}^{\dagger}\left(k_{2}\right) \hat{c}_{\downarrow \nu_{3}}\left(k_{3}\right) \hat{c}_{\uparrow \nu_{4}}\left(k_{4}\right) .
\end{aligned}
$$

One sees that $\Phi_{\vec{\nu}}(\vec{k})$ is not easily calculated. However, we point out the symmetries of the transformation that $T$ inherits from $\mathcal{C}$ :

$$
\mathcal{C}_{\sigma}=\left(\mathcal{C}_{\sigma}^{R}\right)^{*}
$$


where the $R$ operation reverses the indices: $\left(A^{R}\right)_{j m}:=A_{5-j, 5-m}$. For our choice $\omega_{\uparrow n}=\omega_{\downarrow n}$, this leads to

$$
T_{\sigma, j, n}=T_{\sigma, 5-j, n}^{*} .
$$

Furthermore, the definition

$$
\mathcal{C}_{\downarrow}=\left.\mathcal{C}_{\uparrow}\right|_{E_{1} \leftrightarrow E_{2}}
$$

and the fact that the single-particle bands $\omega_{n}$ are invariant under the change $E_{1} \leftrightarrow E_{2}$, we get

$$
T_{\downarrow}=\left.T_{\uparrow}\right|_{E_{1} \leftrightarrow E_{2}} .
$$

\subsection{Boltzmann equation}

This section introduces the Boltzmann equation we will use to find the time evolution of the quasiparticle momentum distribution $n_{\sigma m}(k)=\left\langle\hat{n}_{\sigma m}(k)\right\rangle$. We will revisit the linearization of the Boltzmann equation, which was elaborately done for the general case in Sec. 2.2. It is important to keep in mind the resulting linear operator and the meaning of its eigenvalues, eigenfunctions, and corresponding states-space operators.

We investigate the model parameter $\Delta=4$, which was a preliminary result of the ab-initio calculation of Rajpurohit and Blöchl (2016). The most realistic parameter for $\operatorname{Pr}_{0.5} \mathrm{Ca}_{0.5} \mathrm{MnO}_{3}$ were investigated by Köhler et al. (2016). By the time these new results were found, this chapter has already be finalized and they were not taken into consideration for this thesis. As we will show, this choice $\Delta=4$ constitutes an interesting case, because the linearized Boltzmann equation contains the maximum number of conserved quantities, which we have seen in our calculations, but $\Delta$ is still not overly large.

Furthermore, every manganite $\mathrm{Mn}^{3+}$ contributes half a valence electron due to the doping of $\operatorname{Pr}_{0.5} \mathrm{Ca}_{0.5} \mathrm{MnO}_{3}$, see Sec. 4.1.3. Therefore, the Fermi level is located between the lowest and the second lowest band. This means that for $T=0$, the lowest band is completely filled and the other three bands are empty.

Following Chapter 2 (or App. B.1), the Boltzmann equation for $n_{\uparrow m}(k)=$ $n_{\downarrow m}(k)$ is

$$
\begin{aligned}
\dot{n}_{\nu_{1}}\left(k_{1}, t\right)=\frac{2 \pi U^{2}}{\hbar J} & \int_{\mathrm{K}^{3}} d k_{2} d k_{3} d k_{4} \delta\left(\operatorname{kmod}\left(\Delta k_{\vec{k}}\right)\right) \sum_{\substack{\nu_{2}, \nu_{3}, \nu_{4} \\
\in\{1, \ldots, 4\}}} \delta\left(\Delta \omega_{\vec{\nu}, \vec{k}}\right)\left|\Phi_{\vec{\nu}, \vec{k}}\right|^{2} \\
\times & \{\underbrace{\left[1-n_{\nu_{1}}\left(k_{1}, t\right)\right]\left[1-n_{\nu_{2}}\left(k_{2}, t\right)\right] n_{\nu_{3}}\left(k_{3}, t\right) n_{\nu_{4}}\left(k_{4}, t\right)}_{\text {gain term }} \\
& \underbrace{-n_{\nu_{1}}\left(k_{1}, t\right) n_{\nu_{2}}\left(k_{2}, t\right)\left[1-n_{\nu_{3}}\left(k_{3}, t\right)\right]\left[1-n_{\nu_{4}}\left(k_{4}, t\right)\right]}_{\text {loss term }}\} .
\end{aligned}
$$

Here, we defined the momentum change $\Delta k_{\vec{k}}=\operatorname{kmod}\left(k_{1}+k_{2}-k_{3}-k_{4}\right)$ and the total energy change $\Delta \omega_{\vec{\nu}, \vec{k}}:=\omega_{\nu_{1}}\left(k_{1}\right)+\omega_{\nu_{2}}\left(k_{2}\right)-\omega_{\nu_{3}}\left(k_{3}\right)-\omega_{\nu_{4}}\left(k_{4}\right)$. This Boltzmann equation treats more than one band in contrast to the single-band 
Boltzmann equation that we used in the case of the Hubbard model, see Chapter 3 .

In order to linearize the Boltzmann equation, we define the perturbation $\phi_{\nu}(k, t)$ of the thermal distribution $f_{\nu}(k)$ so that

$$
\begin{aligned}
n_{\nu}(k, t) & =\frac{1}{\exp \left[\beta\left(\omega_{\nu}(k)-\mu\right)-\phi_{\nu}(k, t)\right]+1} \\
& =f_{\nu}(k)-f_{\nu}(k)\left[1-f_{\nu}(k)\right] \phi_{\nu}(k, t)+\mathcal{O}\left(\phi_{\nu}(k, t)^{2}\right) .
\end{aligned}
$$

This leads to the linearized version of the Boltzmann equation (see App. B.2):

$$
\dot{\phi}_{\nu_{1}}\left(k_{1}, t\right)=\hat{\mathcal{L}}[\phi]_{\nu_{1}}\left(k_{1}, t\right)
$$

with the linearized Boltzmann operator

$$
\begin{aligned}
\hat{\mathcal{L}}[\phi]_{\nu_{1}}\left(k_{1}, t\right)= & \frac{2 \pi U^{2}}{\hbar J} \int_{\mathbb{K}^{3}} d k_{2} d k_{3} d k_{4} \delta\left(\Delta k_{\vec{k}}\right) \sum_{\substack{\nu_{2}, \nu_{3}, \nu_{4} \\
\in\{1, . ., 4\}}} F_{\vec{\nu}, \vec{k}}\left|\Phi_{\vec{\nu}, \vec{k}}\right|^{2} \\
& \times \delta\left(\Delta \omega_{\vec{\nu}, \vec{k}}\right)\left[\phi_{\nu_{1}}\left(k_{1}, t\right)+\phi_{\nu_{2}}\left(k_{2}, t\right)-\phi_{\nu_{3}}\left(k_{3}, t\right)-\phi_{\nu_{4}}\left(k_{4}, t\right)\right] .
\end{aligned}
$$

Hereby, we defined

$$
F_{\vec{\nu}, \vec{k}}=\frac{\left[1-f_{\nu_{2}}\left(k_{2}\right)\right] f_{\nu_{3}}\left(k_{3}\right) f_{\nu_{4}}\left(k_{4}\right)}{f_{\nu_{1}}\left(k_{1}\right)} .
$$

\subsubsection{Eigenvalues and eigenfunctions of the linearized Boltz- mann operator}

In App. B.3, we show that the eigenvalues of $\hat{\mathcal{L}}$ are positive, see Eq. (B.72), and that they are the relaxation rates:

$$
\phi_{\nu}(k, t) \stackrel{(B .76)}{=} \sum_{j} A_{j}(0) e^{-\lambda_{j} t} \chi_{\nu}^{(j)}(k) .
$$

Extending the numerical code used in Chapter 3, we calculate the eigenvalues and eigenfunctions of $\left(\hbar J / 2 \pi U^{2}\right) \hat{\mathcal{L}}$, i.e. the operator $\hat{\mathcal{L}}$ without its prefactor. This prefactor can be rewritten using a time constant $t_{0}:=\hbar / 2 \pi J=0.21 \mathrm{fs}$ and the interaction scalar $u=U / J$.

The eigenfunctions $\chi_{\nu}^{(j)}(k)$ of $\hat{\mathcal{L}}$ give information about the quantities that decay with a relaxation rate that is equal to the corresponding eigenvalue. While the eigenvalues answer the question of how fast these quantities are decaying, the eigenfunctions are key to the question of what is decaying. For example, the quantity corresponding to the eigenfunction $\omega_{\nu}(k)$ is the energy. The energy is not decaying, which is consistent with the fact that the corresponding eigenvalue is zero. 


\subsubsection{Numerical calculation of the linearized Boltzmann operator}

In this section, we describe the numerical evaluation of the linearized Boltzmann operator $\hat{\mathcal{L}}$. The numerical code for obtaining the eigenvalues and eigenfunctions has additional complications for the evaluation of the energy conservation $\delta\left(\Delta \omega_{\vec{\nu}, \vec{k}}\right)$. The band structure we use for this model has to be treated differently than the dispersion relation in Sec. 3.2.3. For the latter, we found that there is a unique solution for a certain parametrization of the energy conservation. However, for the dispersion relations of the effective PCMO model, we do not know how many solutions of $\Delta \omega_{\vec{\nu}, \vec{k}}=0$ we can expect. Therefore, we have to scan every momentum $k_{1}, k_{2}$, and $k_{3}$ over its whole value range (note that $k_{4}$ is fixed by momentum conservation). This not only prolongates the time the evaluation takes, but it also requires an extension of the numerical code in the following way: First, we create a grid in the parameter space. Then, for every point, we calculate the energy change $\Delta \omega_{\vec{\nu}, \vec{k}}$. If the sign changes, the point lies near a zero of $\Delta \omega_{\vec{\nu}, \vec{k}}$. The precise position of the zero is found by a Newton-Raphson root-finding procedure adapted to our problem. In our case, the parameter space is two dimensional: $k_{1}$ is fixed, because it is a parameter of $\hat{\mathcal{L}}$. $k_{4}=k_{1}+k_{2}-k_{3}$ is fixed due to momentum conservation. This leaves us with the two degrees of freedom $k_{2}$ and $k_{3}$. The energy conservation restricts this space to one dimension. We obtain paths in $\left(k_{2}, k_{3}\right)$-space where $\Delta \omega_{\vec{\nu}, \vec{k}}$ is zero. Along these paths $\vec{\gamma}(x)=\left(k_{2}(x), k_{3}(x)\right)$, we calculate the path integral. Of course, we can only find some path points. The number of these points is defined by $N_{\vec{\gamma}}$. The rest of the path is interpolated. In App. D.1, we derive the reformulation of the energy conserving delta using paths. The result is

$$
\begin{aligned}
& \int d k_{2} d k_{3} \delta\left(\Delta \omega_{\vec{\nu}, \vec{k}}\right) G_{\vec{\nu}}(\vec{k}) \\
& =\int d x \frac{\left\|\vec{\gamma}^{\prime}(x)\right\| G_{\vec{\nu}}\left(k_{1}, \gamma_{1}(x), \gamma_{2}(x), k_{1}+\gamma_{1}(x)-\gamma_{2}(x)\right)}{\left\|\vec{\nabla}_{\vec{\gamma}}\left(\Delta \omega_{\vec{\nu}, k_{1}, \gamma_{1}(x), \gamma_{2}(x), k_{1}+\gamma_{1}(x)-\gamma_{2}(x)}\right)\right\|},
\end{aligned}
$$

where $G_{\vec{\nu}}(\vec{k})$ is a placeholder for the rest of the integrand of $\hat{\mathcal{L}}$. This leads to

$$
\begin{aligned}
& \hat{\mathcal{L}}[\phi]_{\nu_{1}}\left(k_{1}, t\right)=\frac{2 \pi U^{2}}{\hbar J} \sum_{\vec{\gamma}} \int d x \frac{\left\|\vec{\gamma}^{\prime}(x)\right\|}{\left\|\vec{\nabla}_{\vec{\gamma}} \Delta \omega_{\vec{\nu}, \vec{k}}\right\|} \sum_{\substack{\nu_{2}, \nu_{3}, \nu_{4} \\
\in\{1, \ldots, 4\}}} F_{\vec{\nu}, \vec{k}}\left|\Phi_{\vec{\nu}, \vec{k}}\right|^{2} \\
& \times\left[\phi_{\nu_{1}}\left(k_{1}, t\right)+\phi_{\nu_{2}}\left(k_{2}, t\right)-\phi_{\nu_{3}}\left(k_{3}, t\right)-\phi_{\nu_{4}}\left(k_{4}, t\right)\right] \mid \begin{array}{l}
\left(k_{2}, k_{3}\right)=\vec{\gamma}(x) \\
k_{4}=k_{1}+k_{2}-k_{3}
\end{array}
\end{aligned} .
$$

The integral over $x$ is discretized by the positions of the zeros, which make up the paths $\vec{\gamma}$. Furthermore, we descretize the perturbation $\phi_{\nu}(k, t)$ by the interpolation scheme in App. B.5, i.e. $\phi_{\nu}(k, t)=\sum_{j} B_{j}(k) \phi_{\nu}\left(k_{j}, t\right)$. Hence, we 
obtain

$$
\begin{aligned}
& \hat{\mathcal{L}}[\phi]_{\nu_{1}}\left(k_{1}, t\right) \approx \frac{2 \pi U^{2}}{\hbar J} \sum_{\vec{\gamma}} \sum_{l=1}^{N_{\vec{\gamma}}} \frac{\Delta x\left\|\vec{\gamma}^{\prime}\left(x_{l}\right)\right\|}{\left\|\vec{\nabla} \vec{\gamma} \Delta \omega_{\vec{\nu}, \vec{k}}\right\|} \sum_{\substack{\nu_{2}, \nu_{3}, \nu_{4} \\
\in\{1, ., 4\}}} F_{\vec{\nu}, \vec{k}}\left|\Phi_{\vec{\nu}, \vec{k}}\right|^{2} \sum_{\nu_{5}, j} \phi_{\nu_{5}, j}(t) \\
& \times\left.\sum_{j}\left[\delta_{\nu_{5} \nu_{1}} B_{j}\left(k_{1}\right)+\delta_{\nu_{5} \nu_{2}} B_{j}\left(k_{2}\right)-\delta_{\nu_{5} \nu_{3}} B_{j}\left(k_{3}\right)-\delta_{\nu_{5} \nu_{4}} B_{j}\left(k_{4}\right)\right]\right|_{\substack{\left(k_{2}, k_{3}\right)=\vec{\gamma}\left(x_{l}\right) \\
k_{4}=k_{1}+k_{2}-k_{3}}},
\end{aligned}
$$

where $\phi_{\nu, j}(t)=\phi_{\nu}\left(k_{j}, t\right)$. The distance $\Delta x$ between the discretized path parameters $x$ is compensated by $\left\|\vec{\gamma}^{\prime}\left(x_{l}\right)\right\|$. We can see this when we plug in the approximation $\vec{\gamma}^{\prime}\left(x_{l}\right)=\frac{1}{\Delta t} \sum_{m=a}^{b} C_{m} \vec{\gamma}\left(x_{l+m}\right)$ from App. D.2. The linear operator becomes

$$
\begin{aligned}
& \hat{\mathcal{L}}[\phi]_{\nu_{1}}\left(k_{1}, t\right) \approx \frac{2 \pi U^{2}}{\hbar J} \sum_{\vec{\gamma}} \sum_{l=1}^{N_{\vec{\gamma}}} \frac{\left\|\sum_{m=a}^{b} C_{m} \vec{\gamma}\left(x_{l+m}\right)\right\|}{\left\|\vec{\nabla} \vec{\gamma} \Delta \omega_{\vec{\nu}, \vec{k}}\right\|} \sum_{\substack{\nu_{2}, \nu_{3}, \nu_{4} \\
\in\{1, ., 4\}}} F_{\vec{\nu}, \vec{k}}\left|\Phi_{\vec{\nu}, \vec{k}}\right|^{2} \sum_{\nu_{5}, j} \phi_{\nu_{5}, j}(t) \\
& \times\left.\left[\delta_{\nu_{5} \nu_{1}} B_{j}\left(k_{1}\right)+\delta_{\nu_{5} \nu_{2}} B_{j}\left(k_{2}\right)-\delta_{\nu_{5} \nu_{3}} B_{j}\left(k_{3}\right)-\delta_{\nu_{5} \nu_{4}} B_{j}\left(k_{4}\right)\right]\right|_{\substack{\left(k_{2}, k_{3}\right)=\vec{\gamma}\left(x_{l}\right) \\
k_{4}=k_{1}+k_{2}-k_{3}}} .
\end{aligned}
$$

From this, we can extract a discretized version of $\hat{\mathcal{L}}$ using

$$
\hat{\mathcal{L}}[\phi]_{\nu_{1}}\left(\kappa_{n}, t\right) \approx \frac{2 \pi U^{2}}{\hbar J} \sum_{\nu_{5}=1}^{4} \sum_{j=1}^{N_{\mathrm{k}}} \mathcal{L}_{\nu_{1}, n ; \nu_{5}, j} \phi_{\nu_{5}, j}(t)
$$

The discretized momenta $\kappa_{n}=-\frac{1}{2}+\frac{n}{N_{\mathrm{k}}} \in \mathbb{D}$ are defined the same as for the Hubbard model, see Eq. (3.86). One finds

$$
\begin{aligned}
& \mathcal{L}_{\nu_{1}, n ; \nu_{5}, j}=\sum_{\vec{\gamma}} \sum_{l=1}^{N_{\vec{\gamma}}} \frac{\left\|\sum_{m=a}^{b} C_{m} \vec{\gamma}\left(x_{l+m}\right)\right\|}{\left\|\vec{\nabla} \vec{\gamma} \Delta \omega_{\vec{\nu}, \vec{k}}\right\|} \sum_{\substack{\nu_{2}, \nu_{3}, \nu_{4} \\
\in\{1, \ldots, 4\}}} F_{\vec{\nu}, \vec{k}}\left|\Phi_{\vec{\nu}, \vec{k}}\right|^{2} \\
& \times\left[\delta_{\nu_{5} \nu_{1}} B_{j}\left(k_{1}\right)+\delta_{\nu_{5} \nu_{2}} B_{j}\left(k_{2}\right)-\delta_{\nu_{5} \nu_{3}} B_{j}\left(k_{3}\right)-\delta_{\nu_{5} \nu_{4}} B_{j}\left(k_{4}\right)\right] \mid \begin{array}{l} 
\\
k_{1}=\kappa_{n} \\
\left(k_{2}, k_{3}\right)=\vec{\gamma}\left(x_{l}\right) \\
k_{4}=k_{1}+k_{2}-k_{3}
\end{array}
\end{aligned}
$$

In order to avoid divergences caused by $\left\|\vec{\nabla}_{\vec{\gamma}} \Delta \omega_{\vec{\nu}, \vec{k}}\right\|$, we use the mollification procedure from Eq. (3.91):

$$
\sqrt{\left\|\vec{\nabla}_{\vec{\gamma}} \Delta \omega_{\vec{\nu}, \vec{k}}\right\|^{2}+\left(5 / N_{\mathrm{k}}\right)^{2}} \stackrel{N_{\mathrm{k}} \rightarrow \infty}{\longrightarrow}\left\|\vec{\nabla}_{\vec{\gamma}} \Delta \omega_{\vec{\nu}, \vec{k}}\right\|
$$


The resulting discretized version of $\hat{\mathcal{L}}$ is

$$
\begin{aligned}
& \mathcal{L}_{\nu, m ; \nu_{5}, n}=\sum_{\vec{\gamma}} \sum_{l=1}^{N_{\vec{\gamma}}} \frac{\left\|\sum_{m=a}^{b} C_{m} \vec{\gamma}\left(x_{l+m}\right)\right\|}{\sqrt{\left\|\vec{\nabla}_{\vec{\gamma}} \Delta \omega_{\vec{\nu}, \vec{k}}\right\|^{2}+\left(5 / N_{\mathrm{k}}\right)^{2}}} \sum_{\substack{\nu_{2}, \nu_{3}, \nu_{4} \\
\in\{1, ., 4\}}} F_{\vec{\nu}, \vec{k}}\left|\Phi_{\vec{\nu}, \vec{k}}\right|^{2} \\
& \times\left[\delta_{\nu_{5} \nu_{1}} B_{j}\left(k_{1}\right)+\delta_{\nu_{5} \nu_{2}} B_{j}\left(k_{2}\right)-\delta_{\nu_{5} \nu_{3}} B_{j}\left(k_{3}\right)-\delta_{\nu_{5} \nu_{4}} B_{j}\left(k_{4}\right)\right] \mid \begin{array}{l} 
\\
k_{1}=\kappa_{n} \\
\left(k_{2}, k_{3}\right)=\vec{\gamma}\left(x_{l}\right) \\
k_{4}=k_{1}+k_{2}-k_{3}
\end{array}
\end{aligned}
$$

We extract the low-lying eigenvalues of $\hat{\mathcal{L}}$ by calculating the spectrum of $\mathcal{L}_{\nu, m ; \nu_{5}, n}$ for various discretizations $N_{\mathrm{k}}$ and extrapolating them to $N_{\mathrm{k}} \rightarrow \infty$.

\subsubsection{Corresponding state-space operators}

Now, we make a connection from the eigenfunctions of $\hat{\mathcal{L}}$ to state-space operators. As shown in Sec. 2.4, we can define operators in the state space, whose expectation values converge exponentially in time, where the convergence rate is one of the eigenvalues of $\hat{\mathcal{L}}$. These operators can help finding a physical interpretation for the eigenfunctions and their decay time.

In order to see this, we first consider the scalar product

$$
\left\langle\phi, \chi^{(i)}\right\rangle_{\mathrm{F}}=\sum_{j} A_{j}(t) e^{-\lambda_{j} t}\left\langle\chi^{(j)}, \chi^{(i)}\right\rangle_{\mathrm{F}} \stackrel{(B .71)}{=}\left\|\chi^{(i)}\right\|_{\mathrm{F}}^{2} A_{i}(t) e^{-\lambda_{i} t}
$$

Using this, we can calculate the expectation values of the observables $\hat{I}_{i}:=$ $\int d k \sum_{\nu} \chi_{\nu}^{(i)}(k) \hat{n}_{n}(k)$ :

$$
\begin{aligned}
& \left\langle\hat{I}_{i}\right\rangle_{t}=\int d k \sum_{\nu} \chi_{\nu}^{(i)}(k) n_{\nu}(k, t) \\
& =\int d k \sum_{n} \chi_{\nu}^{(i)}(k) f_{\nu}(k)+\int d k \sum_{n} \chi_{\nu}^{(i)}(k) f_{\nu}(k)\left[1-f_{\nu}(k)\right] \phi_{\nu}(k, t)+\mathcal{O}\left(\phi^{2}\right) \\
& =\int d k \sum_{n} \chi_{\nu}^{(i)}(k) f_{\nu}(k)+\left\langle\chi^{(i)}, \phi\right\rangle_{\mathrm{F}}+\mathcal{O}\left(\phi^{2}\right) \\
& =\left\|\chi^{(i)}\right\|_{\mathrm{F}}^{2} A_{i}(t) e^{-\lambda_{i} t}+C_{i}+\mathcal{O}\left(\phi^{2}\right),
\end{aligned}
$$

with the time-independent constant $C_{i}:=\int d k \sum_{n} \chi_{\nu}^{(i)}(k) f_{\nu}(k)$. Hence, the time evolution of $\hat{I}_{i}$ resembles the one of the related eigenfunction $\chi_{\nu}^{(i)}(k)$. For example, the operator related to the constant function $\phi_{\nu}(k)=1$ is the total number of particles $\hat{N}=\int d k \sum_{n} \hat{n}_{n}(k)$.

It is important to note that our method cannot distinguish between $\hat{I}_{i}$ and $\hat{I}_{i}+\mathcal{O}(U)$ because of the $U \rightarrow 0$ limit used for the derivation of the Boltzmann equation. The strength of the interaction $U$ only enters in the prefactor of $\mathcal{I}_{\text {coll }}$ and $\hat{\mathcal{L}}$. Thus, the eigenfunctions are not altered when changing $U$. 


\subsubsection{Eigenfunctions with eigenvalue zero and correspond- ing conserved quantities}

Zero (and approximately zero) eigenvalues are special as their corresponding contributions to the perturbation $\phi_{\nu}(k, t)$ do not (or hardly) decay. For the model, we investigate in this chapter, there are a number of exactly zero eigenvalued eigenfunctions. The most obvious ones are the constant function $\chi_{\nu}^{(1)}(k)=1$ and $\chi_{\nu}^{(2)}(k)=\omega_{\nu}(k)$. For the particular choice of $\Delta=4$, there are a couple of additional eigenvalues zero. In total, the functions

$$
\begin{aligned}
& \left(\chi_{\nu}^{(1)}(k)\right)_{\nu=1, . ., 4}=(1,1,1,1) \forall k \\
& \left(\chi_{\nu}^{(2)}(k)\right)_{\nu=1, \ldots, 4}=\left(\omega_{1}(k), \omega_{2}(k), \omega_{3}(k), \omega_{4}(k)\right) \forall k \\
& \left(\chi_{\nu}^{(3)}(k)\right)_{\nu=1, . ., 4}=(1,0,1,0) \forall k \\
& \left(\chi_{\nu}^{(+)}(k)\right)_{\nu=1, . ., 4}=(0,0,1,1) \forall k
\end{aligned}
$$

as well as combinations of them like

$$
\begin{aligned}
& \left(\chi_{\nu}^{(+b)}(k)\right)_{\nu=1, \ldots, 4}:=\left(\chi_{\nu}^{(1)}(k)-\chi_{\nu}^{(+)}(k)\right)_{\nu=1, \ldots, 4}=(1,1,0,0) \forall k \\
& \left(\chi_{\nu}^{(3 b)}(k)\right)_{\nu=1, \ldots, 4}:=\left(\chi_{\nu}^{(+b)}(k)-\chi_{\nu}^{(3)}(k)\right)_{\nu=1, . ., 4}=(0,1,0,1) \forall k \\
& \left(\chi_{\nu}^{(3 c)}(k)\right)_{\nu=1, . .4}:=\left(\chi_{\nu}^{(3)}(k)-\chi_{\nu}^{(+)}(k)\right)_{\nu=1, . ., 4}=(1,0,0,-1) \forall k \\
& \left(\chi_{\nu}^{(3 d)}(k)\right)_{\nu=1, \ldots, 4}:=\left(\chi_{\nu}^{(+b)}(k)-\chi_{\nu}^{(3)}(k)\right)_{\nu=1, \ldots, 4}=(0,1,-1,0) \forall k
\end{aligned}
$$

are in the kernel of $\hat{\mathcal{L}}$, i.e. they are eigenfunctions of $\hat{\mathcal{L}}$ corresponding to eigenvalue zero. Obviously, a 2-band calculation is not able to detect $\chi_{\nu}^{(+)}(k)$, which is why we denoted it with a "+" instead of a number. Hence, the 2 -band version of $\hat{\mathcal{L}}$ has 3 eigenfunctions with eigenvalue zero, and the 3 - and 4 -band calculations have 4 of them. This is confirmed by our numerical evaluation, see Fig. 4.11.

There is one more combination of the eigenfunctions with eigenvalue zero that we would like to mention. Defining the mean values $\bar{\phi}_{n}:=\int d k \phi_{\nu}(k)$, one finds $\bar{\omega}_{1}=-\bar{\omega}_{4}$ and $\bar{\omega}_{2}=-\bar{\omega}_{3}$, since $\omega_{1}(k)$ is the mirror image of $\omega_{4}(k)$ using the mirror axis $\phi(k) \equiv 0$. The same holds for $\omega_{2}(k)$ and $\omega_{3}(k)$. Thus,

$$
\begin{aligned}
\left(\chi_{\nu}^{(2 b)}(k)\right)_{\nu=1, \ldots, 4}: & =\left(\chi_{\nu}^{(2)}(k)-\bar{\omega}_{1} \chi_{\nu}^{(3 c)}(k)-\bar{\omega}_{2} \chi_{\nu}^{(3 d)}(k)\right)_{\nu=1, . .4} \\
& =\left(\omega_{1}(k)-\bar{\omega}_{1}, \omega_{2}(k)-\bar{\omega}_{2}, \omega_{3}(k)-\bar{\omega}_{3}, \omega_{4}(k)-\bar{\omega}_{4}\right) \\
\Rightarrow \chi_{\nu}^{(2 b)}(k) & =\omega_{\nu}(k)-\bar{\omega}_{\nu}
\end{aligned}
$$

also corresponds to a zero eigenvalue.

The corresponding state-space operators $\hat{I}_{i}=\int d k \sum_{n} \chi_{\nu_{n}}^{(i)}\left(k_{n}\right) \hat{n}_{n}(k)$ of the zero eigenvalues are

$$
\begin{aligned}
& \hat{I}_{1}=\hat{N}, \quad \hat{I}_{2}=\hat{H}_{0}, \quad \hat{I}_{2 b}=\hat{H}_{0}-\sum_{j} \bar{\omega}_{j} \hat{N}_{j}, \\
& \hat{I}_{3}=\hat{N}_{1}+\hat{N}_{3}, \quad \hat{I}_{3 b}=\hat{N}_{2}+\hat{N}_{4}, \quad \hat{I}_{3 c}=\hat{N}_{1}-\hat{N}_{4}, \\
& \hat{I}_{3 d}=\hat{N}_{2}-\hat{N}_{3}, \quad \hat{I}_{+}=\hat{N}_{3}+\hat{N}_{4}, \quad \hat{I}_{+b}=\hat{N}_{1}+\hat{N}_{2} \text {, }
\end{aligned}
$$


where $\hat{N}_{\nu}$ is the number of particles in the $\nu^{\text {th }}$ band. Their expectation value is conserved for our choice of the model parameters. Furthermore, we can easily read off their physical interpretation: For example $\hat{I}_{1}$ corresponds to the total number of particles and $\hat{I}_{2}$ to the total energy.

\subsection{Result: The lowest non-zero eigenvalue and its eigenfunction}

With this last part of the chapter, we show the results obtained for the effective PCMO model using the Boltzmann-equation approach. We will discuss the thermalization rates and then consider the eigenfunction of the lowest eigenvalue.

In Fig. 4.11, we can see the lowest relaxation rates of the momentum distribution of the PCMO chain. The effect of the highest bands become apparent at inverse temperatures of $\beta \lesssim 3$. For lower temperatures, the lines lie perfectly on top of each other. Furthermore, it is worth noting that the effect of the higher bands start at the same point for the 3-band and the 4-band calculations. Moreover, the semi-logarithmic diagram shows lines for the eigenvalues for low temperatures. This means that, there, the lowest relaxation rates all decay exponentially in inverse final temperature $\beta$. The plot also shows that the eigenvalues depend very strongly on $\beta$. For example, in the low-temperature region, the inverse temperature $\tilde{\beta}=\beta+5$ has a relaxation rate that is two orders of magnitude smaller than the inverse temperature $\beta$. Hence, for reasonably low temperature, the Boltzmann equation does not lead to thermalization within experimentally accessible time.

To get a feeling for the relaxation times $t_{n}=1 / \lambda_{n}$, we give some examples. For a typical hopping rate $J=0.5 \mathrm{eV}$, the time constant is $t_{0} \approx 0.2 \mathrm{fs}$. Hence, for a typical interaction strength $U=3 J$, on the left hand side, the dotted lines approach $t_{n} \approx(1 / 0.2) \cdot t_{0} / u^{2} \approx 0.1 \mathrm{fs}$, the dashed lines $t_{n} \approx(1 / 0.04) \cdot t_{0} / u^{2} \approx$ $0.6 \mathrm{~ns}$ and the solid ones $t_{n} \approx 10^{3} t_{0} / u^{2} \approx 20 \mathrm{fs}$. At room temperature $(\approx 300 \mathrm{~K})$, the dimensionless inverse temperature is $\beta=J /\left(300 \mathrm{~K} \cdot k_{\mathrm{B}}\right) \approx 23$. In this case, the largest finite relaxation time $t_{4}=10^{12} t_{0} / u^{2} \approx 20 \mathrm{~ms}$ is extremely long.

As for the eigenvalues, the most interesting eigenfunction in terms of thermalization is $\chi_{\nu}^{(4)}(k)$. It corresponds to the lowest non-zero eigenvalue $\lambda_{4}$. Hence, the contribution of $\chi_{\nu}^{(4)}(k)$ to the perturbation $\phi_{\nu}(k)$ lives the longest. Note that, as mentioned before, the 3- and 4-band calculations have an additional eigenfunction $\chi_{\nu}^{(+)}(k)$, with eigenvalue $\lambda_{+}=0$. In the middle of Fig. 4.12, the eigenfunction $\chi_{\nu}^{(4)}(k)$ is plotted for a 4-band calculation. In either case, the discretization of momentum space is $N_{\mathrm{k}}=300$ points.

As one can see in Fig. 4.12, the eigenfunction changes its nature, but only for the 4 -band calculation. By plotting $\chi_{1}^{(4)}(0)$ as a function of $\beta$, we see that there is a phase transition at $\beta \approx 2$. This can also be seen by plotting the maximum position in the range $k \in\left(-\frac{1}{4}, \frac{1}{4}\right)$ in band $n=1$ (black line with crosses). They drop to about 0.5 due to the glitch seen in the middle picture of Fig. 4.12. 

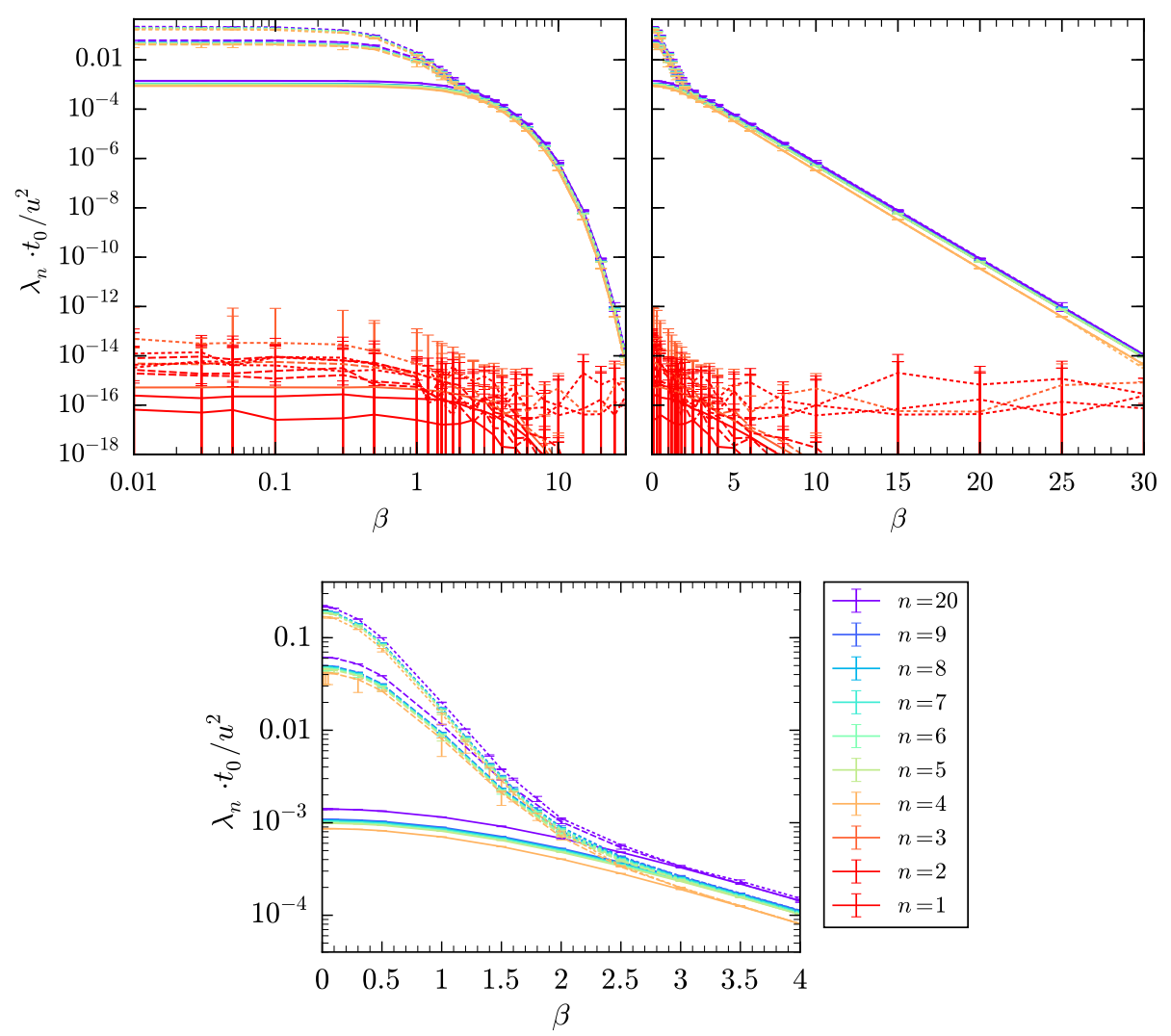

Figure 4.11: Eigenvalues $\left(t_{0} / u^{2}\right) \lambda_{n}$ of $\left(t_{0} / u^{2}\right) \hat{\mathcal{L}}$ as functions of inverse final temperature $\beta$ for $\Delta=4$ with $u=U / J$ and $t_{0}=\hbar /(2 \pi J)$. All three pictures include the results of calculations using 2 bands (solid lines), 3 bands (dashed lines), and 4 bands (dotted lines). The Fermi energy lies between the first and the second band. The figures show the eigenvalues $\lambda_{n}$ with $n \in\{1,2,3,4,5,6,7,8,9,20\}$ as function of $\beta$ and for $N_{\mathrm{b}}=3,4$ the additional eigenvalue $\lambda_{+}$. The red, fluctuating lines describe the eigenvalues $\lambda_{1,2,3}$ and $\lambda_{+}$, which are zero analytically. Their values give our numerical precision and are $\lesssim 10^{-13}$. They give the resolution of the eigenvalues by our numerical calculation. As expected, these eigenvalues are extremely small. Note that $\lambda_{+}$does not appear in the legend, but is added in the plot. The error bars of the eigenvalues have two contributions. The first is determined by the extrapolation error when finding the limit $L \rightarrow \infty$. The second applies only to the non-zero eigenvalues $\lambda_{n} \geqslant 4$ and is given by the numerical resolution $\max _{n \in\{1,2,3,+\}}\left|\lambda_{n}\right|$.

The top-right graph is a semi-logarithmic plot and shows that the eigenvalues decay exponentially as functions of $\beta$. The diagram on the upper left displays the same eigenvalues as a double-logarithmic plot. Here, one can nicely observe the splitting of the eigenvalues for different numbers of bands included in the calculations. The graph on the bottom zooms into this region. There, one can see that the eigenvalues for different numbers of bands lie on top of each other for $\beta \gtrsim 3$. 

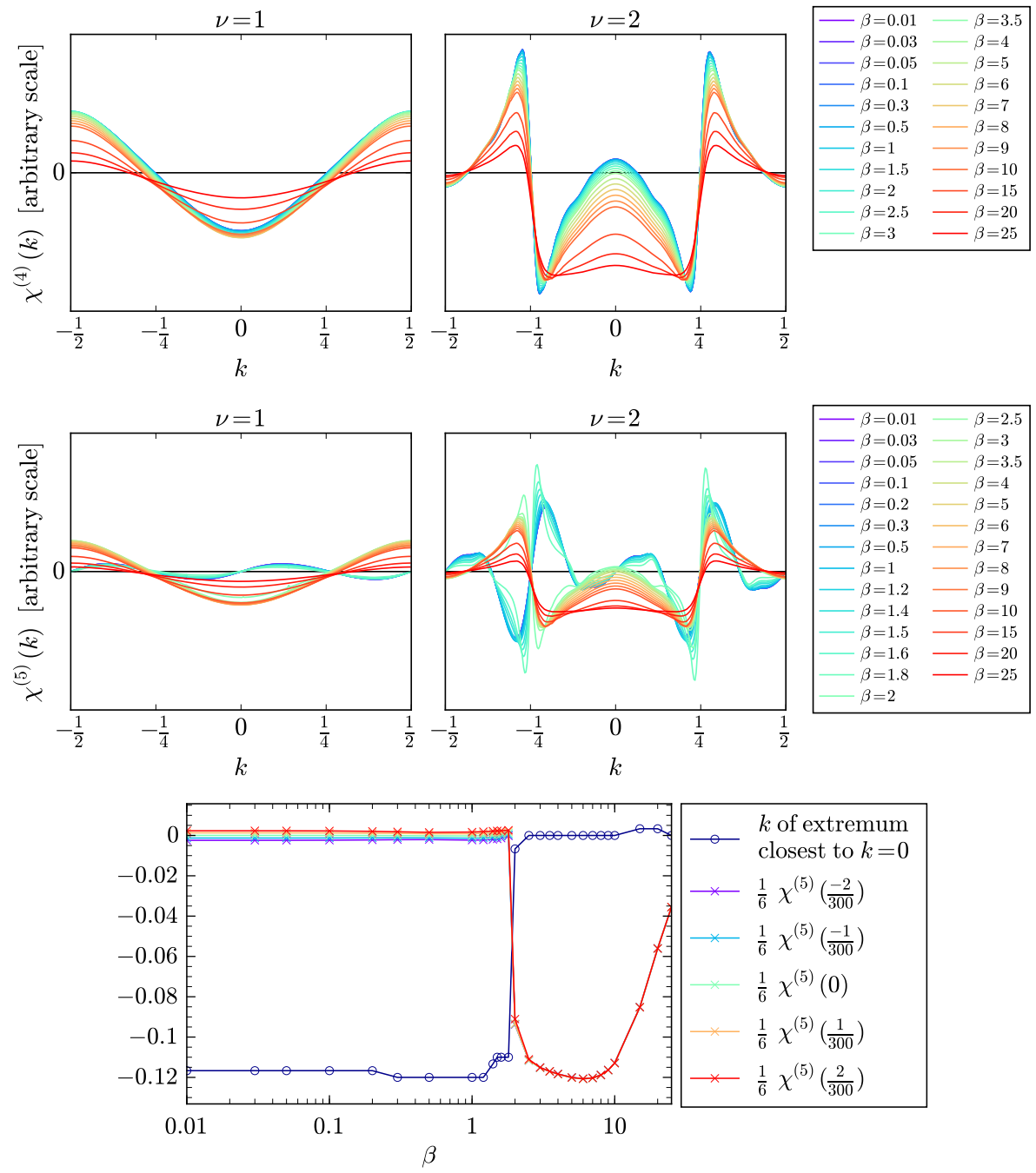

Figure 4.12: Eigenfunctions corresponding to the first non-vanishing eigenvalue for $\Delta=4$ and $N_{\mathrm{k}}=300$. The upper plot shows the result of a 2-band calculation, the middle one the result of a 4-band calculation, where we only show the lower two bands of the eigenfunction, i.e. $\chi_{1}^{(4)}(k)$ and $\chi_{2}^{(4)}(k)$. The eigenfunctions concur for $\beta \gtrsim 2$. In contrast to eigenfunction obtained by the 2 -band calculation, the one obtained by the 4 -band calculation abruptly changes its shape at $\beta \approx 2$. The first-band part quickly goes from having two zeros to having four. Both parts change their symmetry around $k=0$ to an anti-symmetry around $k=0$. This transition is displayed in the bottom picture. It shows the position $k$ of the extremum of $\chi_{1}^{(4)}(k)$ closest to $k=0$, and also $\chi_{1}^{(4)}(k)$ with $k \in\left\{0, \pm 1 / N_{\mathrm{k}}, \pm 2 / N_{\mathrm{k}}\right\}$ as a function of $\beta$. 
The first-band part of $\chi_{1}^{(4)}(0)$ seems to be changing from the total energy current's function $j^{E}(k):=\omega_{1}(k) \omega_{1}^{\prime}(k)$ to the total energy's function $\omega_{1}(k)$, where the $n^{\text {th }}$ band's total energy current operator is

$$
\hat{J}_{n}^{E}=\int d k j^{E}(k) \hat{n}_{n}(k)+\mathcal{O}(U)
$$

and its total energy is $\hat{H}_{n}=\int d k \omega_{n}(k) \hat{n}_{n}(k)+\mathcal{O}(U)$.

The nature of the second band's part $\chi_{2}^{(4)}(0)$ is unknown. This is a good example of how difficult it can be to find a physical meaning for a generic eigenfunction of $\hat{\mathcal{L}}$.

In this chapter, we applied the Boltzmann-equation method to a more realistic model that includes a richer band structure. Thereby, we found the thermalization rates of the quasiparticle momentum distribution of an effective model for a PCMO chain. Furthermore, in Sec. 4.3.4, we gave an interpretation for the eigenvalues that are exactly zero. Their corresponding quantities that are conserved within the Boltzmann equation are linear combinations of the energy and the number operators counting the total number of quasiparticles per band. 


\section{Chapter 5}

\section{Conclusions and outlook}

In this thesis, we aimed for predicting thermalization time scales in one-dimensional many-body quantum systems. As we pointed out in the introduction, thermalization may take a very long time in low dimensional systems. In particular, we investigated the thermalization time of the quasiparticle momentum distribution. The method is a Boltzmann equation and we demonstrated how to derive it. Contrary to common belief, we proved that in one dimension, the Boltzmann equation is able to change the momentum distribution if the dispersion relation is continuous and periodic. In many cases, the Boltzmann equation even leads to thermalization. To be more precise, in the investigation of the Hubbard model we found that the next-to-nearest-neighbor hopping term is responsible for the thermalization in the Boltzmann-equation approach.

To be more specific, we investigated a Hubbard model with nearest-neighbor hopping $J$, next-to-nearest-neighbor hopping $J^{\prime}$, and interaction $U$ in Chapter 3. Thereby, we confirmed the findings of Fürst et al. (2012, 2013b). Specifically, in the integrable case $\left(J^{\prime}=0\right)$, we found that the Boltzmann equation does not lead to thermalization. There, the linearized Boltzmann equation determines all the fixed points of the Boltzmann equation, that were previously identified by Fürst et al. (2012). Furthermore, we also find that in the non-integrable case $\left(J^{\prime} \neq 0\right)$ the additional next-to-nearest-neighbor-hopping term leads to thermalization. Our investigation revealed how the thermalization rate depends on the system parameters $J, J^{\prime}$, and $U$ and the state properties $\beta$ and $\mu$. The final inverse temperature $\beta$ and the final chemical potential $\mu$ are defined so that they describe the thermal state that has the same energy and same amount of electrons as the initial state. We found that

$$
\lambda_{\text {thermalization }}=\tilde{\lambda}_{\text {thermalization }} e^{-\beta(\Gamma+|\mu|)}
$$

for small temperature $1 / \beta$ and the constant $\Gamma \approx 0.86$. It is important to note that the chemical potential $\mu$ is defined so that it is zero in the middle of the single Hubbard band. The prefactor has the form

$$
\tilde{\lambda}_{\text {thermalization }} \propto\left(\frac{U}{J}\right)^{2}\left(\frac{J^{\prime}}{J}\right)^{2}
$$


for small $U / J$ and small $J^{\prime} / J$, see Eq. (3.104). This is consistent with the fact that the Boltzmann equation does neither lead to thermalization in the integrable case $\left(J^{\prime}=0\right)$, nor in the non-interacting case $(U=0)$. Furthermore, we learned that for small temperatures the thermalization is exponentially suppressed in inverse final temperature $\beta$, i.e. the momentum distribution is approximately conserved within the Boltzmann scheme.

Hence, using this linearized Boltzmann equation, we are able to calculate thermalization times analytically. Therefore, it is possible to predict the thermalization times for numerical calculations such as tDMRG, for which it is difficult to unambiguously identify thermalization on the accessible time scales.

An interesting aspect of the linearization method is the ability to distinguish the relaxation times of different contributions of the momentum distribution and link them to operators in the state space. These operators give a physical meaning to their respective relaxation time $1 / \lambda_{n}$, because their expectation values equilibrate at time $t \approx 1 / \lambda_{n}$. In the case of the Hubbard model, e.g., the largest relaxation rate corresponds to the state-space operator

$$
\hat{I}_{3} \approx \hat{K}-\frac{1}{4}\left(\hat{N}_{\mathrm{R}}-\hat{N}_{\mathrm{L}}\right)
$$

which is a combination of the total momentum operator $\hat{K}$ and the number operators of left- and right-moving particles, $\hat{N}_{\mathrm{L}}$ and $\hat{N}_{\mathrm{R}}$ respectively, see Eq. (3.117).

Taking $\hat{I}_{3}$ into account, we found three different relaxation scenarios for small temperatures:

1. Away from half filling: The relaxation rate becomes exponentially small as a function of inverse final temperature $\beta$,

$$
\lambda_{\text {thermalization }} \propto\left(\frac{U}{J}\right)^{2}\left(\frac{J^{\prime}}{J}\right)^{2} e^{-\Gamma_{1} \beta},
$$

with a constant $\Gamma_{1}>0$.

2. Half filling and $\left\langle\hat{I}_{3}\right\rangle \neq 0$ : the relaxation rate is again exponentially small as a function of $\beta$ for low temperature,

$$
\lambda_{\text {thermalization }}=\lambda_{3} \propto\left(\frac{U}{J}\right)^{2}\left(\frac{J^{\prime}}{J}\right)^{2} e^{-\Gamma_{2} \beta},
$$

with another constant $\Gamma_{2}>0$.

3. Half filling and $\left\langle\hat{I}_{3}\right\rangle=0$ : The relaxation rate is hardly dependent on $\beta$. Instead of the exponential in $\beta$, a factor of $\mathcal{O}\left(\beta^{0}\right)$ appears,

$$
\lambda_{\text {thermalization }}=\lambda_{4} \propto\left(\frac{U}{J}\right)^{2}\left(\frac{J^{\prime}}{J}\right)^{2} .
$$

Remarkably, in the last case, the relaxation rate is orders of magnitudes larger than in the other cases. We explain this behavior with Umklapp processes, which are effective at half filling only. Hence, with half filling, there is the largest chance to see thermalization in experiments and numerical investigations. 
For high temperatures, the relaxation rates are barely dependent on $\beta$. However, one can clearly see that the relaxation rates grow with growing temperature $T$. Since $T$ measures the excitation energy in the system, the relaxation is faster the more excitations there are (as long as $T$ is positive). This trend is similar in higher-dimensional systems, see e.g. Mueller and Rethfeld (2013).

We then extended our study to the effective one-dimensional model for the material $\mathrm{Pr}_{\mathrm{x}} \mathrm{Ca}_{1-\mathrm{x}} \mathrm{MnO}_{3}$, with $x=1 / 2$. Our investigation revealed that the thermalization time depends strongly on the band structure. There are conserved quantities arising in the Boltzmann approach when the band gaps are increasing. For instance, if a band gap becomes larger than the bandwidths of a two-band system, two-particle scattering cannot change the particle number in any of the two bands because of energy conservation. In our PCMO model, the quantities conserved within the Boltzmann scheme are combinations of the particle numbers $\hat{N}_{\nu}$ of the four bands $\nu=\in\{1,2,3,4\}$. Furthermore, there are a lot of approximately conserved quantities at small temperature. The corresponding relaxation rates are exponentially suppressed in inverse temperature $\beta$ similar to those of the Hubbard model away from half filling. We found, that at room temperature and for an interaction strength $U=3 \mathrm{~J}$, the thermalization takes about $20 \mathrm{~ms}$, which is extremely long. This time is much larger than the experimentally found relaxation time of excitations in the three-dimensional material (Saucke et al., 2012), which is of the order of nanoseconds. Hence, the reason for our long relaxation time may be the low dimensionality of the system.

For a more realistic prediction of electronic thermalization, one could derive a Boltzmann equation in higher dimensions. There, the evaluation is different, because the higher dimensional Boltzmann equation with $d$ spatial dimensions differs in the following way: The number of integrals in the collision term is $3 \cdot d$, the number of constraints due to energy and momentum conservation is $1+d$. Hence, in $d>1$ dimensions, $N_{I}=2 d-1$ integrals are left after integrating out the conservation laws. Thus, in this case, the constraints are not as important as in one dimension. There will always be enough scattering processes that are able to thermalize the momentum distribution for $d>1$. We are not aware of any counter example. In order to evaluate the Boltzmann equation in the $d>1$ cases, one probably needs to exploit this fact by using smart approximations, which are supposed to reduce the number $N_{I}$ of integrals, which are to be evaluated. For example, Kabanov and Alexandrov (2008) simplify a Boltzmann equation using an approximation, in which the particle-hole excitations have an energy much smaller than the Fermi energy. This results in a rate equation, that is independent of the dispersion relation. Note, however, that since this approach does not take the details of the band structure into account, it does not allow for investigating how the band structure effects thermalization.

Another endeavor one could pursue is adding phonons. This would surely accelerate thermalization, but, of course, it is difficult to estimate by how much. One can again simply consider the first-order contribution in the electron-phonon interaction and apply similar approximations like the ones for deriving the Boltzmann equation used in this thesis. However, if there is no thermalization without the phonons, it is not guaranteed that implementing the leading contribution of the phonon Boltzmann equation automatically leads to thermalization.

Furthermore, it would be quite interesting to derive higher-order contributions 
to the time evolution of the quasiparticle momentum distribution. The next correction describes three-particle collisions, the higher corrections correspond to collisions involving more particles. If the collision term of the Boltzmann equation does not vanish, we do not expect a qualitative change of the thermalization behavior. However, if the collision term does not lead to thermalization, then the higher orders determine the thermalization time. Nonetheless, we expect them to be extremely small.

Many aspects about thermalization in quantum systems are still unknown. Quantum mechanics and physics in general keep on to be surprising and people continue to reveal new and fascinating effects. We hope that our contribution will inspire future research endeavors. 


\section{Acknowledgments}

Finally, I arrive at the part where I can thank all the people that helped me in any way through this work. However, it is something very personal, and I think, there should be a part of the informal me in this thesis. Hence - finally, the last word of the sort thus, hence, thence, therefore -, I will write my acknowledgments in German. I know that everyone in my examination committee, my whole family and most of my colleagues and friends know German, but for everyone, who does not - Ebad -, you have a rough estimate of my gratitude to you by looking for your name and counting the number of words until you reach the next name.

\section{Danksagung}

Ich danke zu allererst meinem Doktorvater, Prof. Stefan Kehrein, der es mir mit viel Geduld und unermüdlichem Glauben an meinen Erfolg ermöglicht hat in diesem Bereich zu promovieren. Seine hohen Anforderungen an seine Mitarbeiter haben dazu geführt, dass ich über mich hinausgewachsen bin, wie ich es niemals für möglich gehalten hätte. Ich möchte mich auch sehr dafür bedanken, dass er mir diesen Laptop zur Verfügung gestellt hat, auf dem ich die gesamte Doktorarbeit geschrieben habe, und dass er die vielen Reisen zu Konferenzen finanziert hat. Besonders dankbar bin ich für die zwei Reisen in die USA zum APS March meeting.

Ich bin sehr traurig darüber, dass mein früherer Zweitbetreuer Prof. Thomas Pruschke, der mir als lebensfroher Mensch, der immer eine offene Tür für seine Studenten hatte, in Erinnerung geblieben ist, viel zu früh gestorben ist. Ich bin sehr dankbar, dass Prof. Peter Blöchl ohne Zögern für ihn eingesprungen ist und mir immer eine gute Ansprechperson war.

Auch der restlichen Prüfungskommission möchte ich für ihre Arbeit danken. Ganz besonderen Dank verdient dabei Salvatore Manmana, der immer einer meiner ersten Anlaufstationen bei physikalischen Problemen war.

Außerdem danke ich Mariya Medvedyeva, die nicht nur durch Ihr ungeheueres Wissen über alle Werke von Landau brilliert, sondern auch durch ihre unfassbare Intelligenz. Des Weiteren wäre das Einleben im fernen Göttingen ohne das bayerische Wesen des immer lächelnden und so vortrefflich genannten Oberreuter Johannes nicht halb so schön gewesen. Thank you Ebad for, you know, talking about, you know, stuff. 
Danke für die gute Atmosphäre im Institut und viele Diskussionen, seien sie physikalischer Natur oder nicht, an Stephan Filor, Thomas Köhler, Lorenzo Cevolani, Anneli Landmesser, Davide Fioretto, I. Homrighausen, Beniamin Lenz, Ehsan Irani \& Zahra (Elham) Mokhtari, Reiner Härtle, Mathias Grob, Florian Sohn, Patrick Haase, Debanjan Basu, Mohammadhosein (Navid) Razbin Khalilabad Iran, Abeling, Herr Schmitt und dem restlichen Institut für theoretische Physik.

Ich bedanke mich auch für die wunderbare technische Unterstützung durch Jürgen Holm und Thomas Köhler. Mit diesen beiden war man nie verlassen, abgesehen natürlich von den schlimmen Zeiten, in denen Herr Holm im Urlaub war, und kurz nach seiner Abreise das Netzwerk streikte.

Danke an meinen Kumpel und neuen Chef Stephan Kramer, der sich oft viel Zeit mit mir nahm und nimmt. Vor allem waren das Analysieren meiner Vortragsweise und seine Verbesserungsvorschläge vor meiner Verteidigung äußerst hilfreich. Immer schön ins Publikum schauen - check!

Natürlich will ich mich auch bei den vielen Korrekturlesern bedanken, nämlich Prof. Kurt Schönhammer, Salvatore Manmana, Ebad Kamil, Timo Fischer, Mathias Grob, Thomas Köhler und Sebastian Paeckel. Ganz spezieller Dank geht hierbei an Isa Köhler, die echt dieses ganze riesige Monster gelesen hat und wirklich jedes einzelne Komma verbessert hat. Und ich schreibe Monster, nicht nur weil dieses Schriftstück so unangenehm lang ist, sondern weil sie sich als Geisteswissenschaftlerin durch diese ganze theoretische Physik pflügen musste. Ich hoffe, dass das Schmunzeln über das elektronische Doping und die Gefühle der Elektronen sie über das Schlimmste hinwegtrösten konnte.

Als nächstes danke ich von ganzen Herzen der ersten Vorsitzenden des FabianBiebl-Fanclubs, Maria Reischl-Weitzel. Sie hat schon an mich geglaubt, als ich noch nicht einmal angefangen hatte Physik zu studieren. Sorry, dass es keinen Nobelpreis gab. Ich hoffe, dass der Fanclub trotzdem weiterhin besteht ;)

Ferner danke ich auch der vierten Herrenfußballmannschaft des SC Weende, die mich so freundlich aufgenommen haben und mir dadurch vor allem eine entspanntere Schlussphase der Dissertation ermöglicht haben, und insbesondere unserer Trainerin und einer meiner besten Freundinnen Antje Amman.

Danke an all meine weiteren Freunde, die sich mein Klagen ohne Murren angehört haben: Fabian \& Katrin Hellmundt, Thomas Dürbeck, Tilo Thomaschek, Stephanie Kolodzeyzik, Olivia Schauwienold \& ihrem Moppi, Joachim Weitzel, Sabine Wieland, Werner Schmidt, Johannes Eberl, und allen anderen die ich vergessen habe.

Vielen Dank auch an all meine Verwandten, die sich mein Klagen anhören mussten. Ganz besonders viel mussten neben meinen Eltern auch meine Oma Antonie Biebl, meine Tante Dani Biebl, mein Onkel Bernd Biebl und mein Stiefvater Andreas Trogisch ertragen.

Meiner Quasischwester Julia Eberl möchte ich speziell danken, da sie mich wie eine Schwester durch das Leben begleitet hat, auch in Zeiten, in denen ich sie sträflich vernachlässigt habe. Ich möchte auf diesem Wege auch ihrem Sohn Lorenz Eberl, der kurz nach meiner Verteidigung das Licht des Lebens erblicken durfte, viel Glück und Gesundheit wünschen. 
Auch meinem Vater, Christian Klepsch, schulde ich viel Dank, da er immer nachgefragt, Mut zugesprochen und Beistand geleistet hat. Ihm konnte und kann ich immer alles anvertrauen.

Zu guter Letzt möchte ich mich ganz besonders herzlich bei meiner lieben Mama, Renate Biebl, bedanken. Mehr muss ich da gar nicht schreiben, denn sie erzählt ja auch nicht viel. Na Schmarrn: Ohne sie hätte ich nach spätestens zwei Jahren aufgegeben. Vielen tausend Dank!!! Sie hat mich zu einer selbständigen Person mit großem Durchhaltevermögen erzogen und mir bei Problemen immer verdeutlich, dass wir das schon irgendwie schaffen werden.

Ich hoffe ich habe niemanden vergessen, dem ich Dank schulde. Beschwerden werde ich unter fabian.biebl@gmx.de entgegennehmen und sie in der nächsten Version berücksichtigen.

Diese Arbeit wurde finanziert mit Mitteln des Sonderforschungsbereichs (SFB) 1073 der deutschen Forschungsgemeinschaft (DFG). 


\section{Appendix A}

\section{Appendix for the introduction}

\section{A.1 One-particle Green function for the grand- canonical ensemble}

In this section, we present the Lehmann representation of the correlator containing a creation and annihilation operator, which is known as one-particle Green function or two-point Green function. We follow the known derivations, which can be found for example in the book of Mahan (1990). The results of this section are used in Sec. 1.2.1.

For fermions, the lesser, greater, retarded, and advanced Green functions are defined by

$$
\begin{aligned}
& G^{<}\left(\vec{r}_{1}, \vec{r}_{2}, t_{1}, t_{2}\right):=i\left\langle\hat{c}_{\vec{r}_{2}}^{\dagger}\left(t_{2}\right) \hat{c}_{\vec{r}_{1}}\left(t_{1}\right)\right\rangle \\
& G^{>}\left(\vec{r}_{1}, \vec{r}_{2}, t_{1}, t_{2}\right):=-i\left\langle\hat{c}_{\vec{r}_{1}}\left(t_{1}\right) \hat{c}_{\vec{r}_{2}}^{\dagger}\left(t_{2}\right)\right\rangle \\
& G^{R}\left(\vec{r}_{1}, \vec{r}_{2}, t_{1}, t_{2}\right):=\theta\left(t_{1}-t_{2}\right)\left[G^{>}\left(\vec{r}_{1}, \vec{r}_{2}, t_{1}, t_{2}\right)-G^{<}\left(\vec{r}_{1}, \vec{r}_{2}, t_{1}, t_{2}\right)\right] \\
& G^{A}\left(\vec{r}_{1}, \vec{r}_{2}, t_{1}, t_{2}\right):=\theta\left(t_{2}-t_{1}\right)\left[G^{<}\left(\vec{r}_{1}, \vec{r}_{2}, t_{1}, t_{2}\right)-G^{>}\left(\vec{r}_{1}, \vec{r}_{2}, t_{1}, t_{2}\right)\right] .
\end{aligned}
$$

In thermal equilibrium, the Green functions are translationally invariant in space. Therefore, in this case, all information is contained in the transformed Green functions

$$
\int d^{3} r e^{-i \vec{k} \cdot \vec{r}} G^{X}\left(\overrightarrow{0}, \vec{r}, t_{1}, t_{2}\right) \quad \text { for } X \in\{<,>, R, A\} .
$$

The lesser Green function, Fourier-transformed in space, can be written as

$$
G^{<}\left(\vec{k}, t_{1}, t_{2}\right)=i\left\langle\hat{c}_{\vec{k}}^{\dagger}\left(t_{2}\right) \hat{c}_{\vec{k}}\left(t_{1}\right)\right\rangle .
$$

Now, we express the expectation value by the thermal density matrix $\exp (-\beta \hat{\mathcal{H}})$ with $\hat{\mathcal{H}}=\hat{H}-\mu \hat{N}$, the Hamiltonian $\hat{H}$, and the number operator $\hat{N}$ :

$$
G^{<}\left(\vec{k}, t_{1}, t_{2}\right)=\frac{i}{Z} \operatorname{tr}\left[e^{-\beta \hat{\mathcal{H}}} \hat{c}_{\vec{k}}^{\dagger}\left(t_{2}\right) \hat{c}_{\vec{k}}\left(t_{1}\right)\right] .
$$


As a next step, we rewrite the trace with the help of the eigenfunctions $|n\rangle$ of $\hat{\mathcal{H}}$ and we insert $\mathbb{1}=\sum_{n}|n\rangle\langle n|$ :

$$
G^{<}\left(\vec{k}, t_{1}, t_{2}\right)=\frac{i}{Z} \sum_{m, n}\left\langle n\left|e^{-\beta \hat{\mathcal{H}}} \hat{c}_{\vec{k}}^{\dagger}\left(t_{2}\right)\right| m\right\rangle\left\langle m\left|\hat{c}_{\vec{k}}\left(t_{1}\right)\right| n\right\rangle
$$

Since $\hat{c}_{\vec{k}}^{\dagger}(t)=e^{i \hat{H} t} \hat{c}_{\vec{k}}^{\dagger} e^{-i \hat{H} t}$, one can pull the time dependence out of the matrix elements:

$$
G^{<}\left(\vec{k}, t_{1}, t_{2}\right)=\frac{i}{Z} \sum_{m, n} e^{-\beta K_{n}}\left|\left\langle m\left|\hat{c}_{\vec{k}}\right| n\right\rangle\right|^{2} e^{i\left(t_{2}-t_{1}\right)\left(E_{n}-E_{m}\right)},
$$

where $K_{n}=E_{n}-\mu N_{n}$ are the eigenvalues of $\hat{\mathcal{H}}, E_{n}$ the energy eigenvalues, and $N_{n}$ the eigenvalues of the number operator. Hence, this Green function is only dependent on the time difference $t_{2}-t_{1}$ and we can define the fully Fourier-transformed, lesser Green function

$$
\begin{aligned}
G^{<}(\vec{k}, \omega) & =\int d t e^{i \omega t} G^{<}(\vec{k}, 0, t) \\
& =\frac{i 2 \pi}{Z} \sum_{m, n} e^{-\beta K_{n}}\left|\left\langle m\left|\hat{c}_{\vec{k}}\right| n\right\rangle\right|^{2} \delta\left(\omega+E_{n}-E_{m}\right) .
\end{aligned}
$$

This is the Lehmann representation of the lesser Green function and we can see that it is purely imaginary.

Analogously, one finds for the retarded Green function

$$
\begin{aligned}
G^{R}\left(\vec{k}, t_{1}, t_{2}\right) & =-i \theta\left(t_{1}-t_{2}\right)\left[\left\langle\hat{c}_{\vec{k}}\left(t_{1}\right) \hat{c}_{\vec{k}}^{\dagger}\left(t_{2}\right)\right\rangle+\left\langle\hat{c}_{\vec{k}}^{\dagger}\left(t_{2}\right) \hat{c}_{\vec{k}}\left(t_{1}\right)\right\rangle\right] \\
& =-\frac{i \theta\left(t_{1}-t_{2}\right)}{Z} \sum_{m, n}\left[e^{-\beta K_{n}}+e^{-\beta K_{m}}\right]\left|\left\langle m\left|\hat{c}_{\vec{k}}\right| n\right\rangle\right|^{2} e^{i\left(t_{2}-t_{1}\right)\left(E_{n}-E_{m}\right)} .
\end{aligned}
$$

Now, we perform the Fourier transformation in time

$$
\begin{aligned}
G^{R}(\vec{k}, \omega+i 0) & =\int d t e^{i(\omega+i 0) t} G^{R}(\vec{k}, 0, t) \\
& =\frac{1}{Z} \sum_{m, n} \frac{\left[e^{-\beta K_{n}}+e^{-\beta K_{m}}\right]\left|\left\langle m\left|\hat{c}_{\vec{k}}\right| n\right\rangle\right|^{2}}{\omega+E_{n}-E_{m}+i 0} \lim _{\delta \rightarrow 0^{+}}[\underbrace{\lim _{T \rightarrow \infty} e^{-T \delta}}_{=0}-e^{i \delta}] .
\end{aligned}
$$

The imaginary part of this can be calculated by making use of the Sokhotski-Plemelj theorem (see e.g. Blanchard and Bruening (2003))

$$
\frac{1}{x \pm i 0}=\mathcal{P} \frac{1}{x} \mp i \pi \delta(x)
$$

with the Cauchy principal value $\mathcal{P}$. This leads to the spectral function of the retarded Green function

$$
\begin{aligned}
A(\vec{k}, \omega) & :=-\frac{1}{\pi} \operatorname{Im} G^{R}(\vec{k}, \omega+i 0) \\
& =\frac{1}{Z} \sum_{m, n}\left[e^{-\beta K_{n}}+e^{-\beta K_{m}}\right]\left|\left\langle m\left|\hat{c}_{\vec{k}}\right| n\right\rangle\right|^{2} \delta\left(\omega+E_{n}-E_{m}\right) .
\end{aligned}
$$


By multiplying the spectral function $A(\vec{k}, \omega)$ with the Fermi-Dirac function $f(\omega)=1 /(1+\exp (\beta \omega-\mu))$, we can establish a connection to the lesser Green function $G^{<}(\vec{k}, \omega)$ :

$f(\omega) A(\vec{k}, \omega)=\frac{1}{Z} \sum_{m, n} e^{-\beta K_{n}} f(\omega)\left[1+e^{\beta\left(K_{n}-K_{m}\right)}\right]\left|\left\langle m\left|\hat{c}_{\vec{k}}\right| n\right\rangle\right|^{2} \delta\left(\omega+E_{n}-E_{m}\right)$.

We make use of the fact that the matrix element $\left\langle m\left|\hat{c}_{\vec{k}}\right| n\right\rangle$ vanishes for $N_{m} \neq$ $N_{n}-1$. Therefore, we get

$$
\begin{aligned}
f(\omega) A(\vec{k}, \omega) & =\frac{1}{Z} \sum_{m, n} e^{-\beta K_{n}} f(\omega)\left[1+e^{\beta\left(E_{n}-E_{m}-\mu\right)}\right]\left|\left\langle m\left|\hat{c}_{\vec{k}}\right| n\right\rangle\right|^{2} \delta\left(\omega+E_{n}-E_{m}\right) \\
& =\frac{1}{Z} \sum_{m, n} e^{-\beta K_{n}}\left|\left\langle m\left|\hat{c}_{\vec{k}}\right| n\right\rangle\right|^{2} \delta\left(\omega+E_{n}-E_{m}\right) \\
& =\frac{1}{i 2 \pi} G^{<}(\vec{k}, \omega) .
\end{aligned}
$$

The spectral function is very valuable in this thesis, because it contains the momentum distribution, which we show in the following formula:

$$
\begin{aligned}
n(\vec{k}) & =\left\langle\hat{c}_{\vec{k}}^{\dagger}(0) \hat{c}_{\vec{k}}(0)\right\rangle=\int d t \delta(t)\left\langle\hat{c}_{\vec{k}}^{\dagger}(t) \hat{c}_{\vec{k}}(0)\right\rangle=\frac{1}{i 2 \pi} \int d \omega \int d t e^{i \omega t} G^{<}(\vec{k}, 0, t) \\
& =\frac{1}{i 2 \pi} \int d \omega G^{<}(\vec{k}, \omega)=\int d \omega f(\omega) A(\vec{k}, \omega) .
\end{aligned}
$$




\section{Appendix B}

\section{Multi-band Boltzmann equation}

\section{B.1 Derivation}

This section contains the derivation of a multi-band Boltzmann equation following Erdös et al. (2004), who derived a one-band Boltzmann equation. Note that here we will not consider a matrix-valued Boltzmann equation like Fürst et al. (2013a) did.

A couple of important assumptions for obtaining the Boltzmann equation, which is used in this thesis, are summarized in Eq. (2.14), which reads

$$
\left\langle\hat{c}_{\sigma \nu}^{\dagger}(k) \hat{c}_{\tau \nu}^{\dagger}(q)\right\rangle_{t}=\delta_{\sigma \tau} \delta_{\nu \nu^{\prime}} \delta_{k q}^{D} n_{\sigma \nu}(k, t),
$$

where we defined the Dirac delta $\delta_{x y}^{D}:=\delta(x-y)$ to keep the notation short. The assumptions that lead to Eq. (B.1) have been explained above Eq. (2.14). As Erdös et al. (2004), we will drag the factor $\delta(0)$ along to cancel it later in Eq. (B.37) with the one in $\delta\left(\Delta k_{\vec{k}}\right)^{2}=\delta(0) \delta\left(\Delta k_{\vec{k}}\right)$, where $\Delta k_{\vec{k}}:=\operatorname{kmod}\left(k_{1}+\right.$ $\left.k_{2}-k_{3}-k_{4}\right)$ is the change of momentum. The kmod function is defined in Eq. (2.3).

\section{B.1.1 Quantum-mechanical time evolution}

This derivation assumes a Hamiltonian of the form

$$
\begin{aligned}
\hat{H} & =\hat{H}_{0}+\hat{H}_{\mathrm{int}}, \\
\hat{H}_{0} & =\int d k \sum_{\sigma \nu} \hbar \Omega_{\nu}(k) \hat{n}_{\sigma \nu}(k), \\
\hat{H}_{\mathrm{int}} & =U \int_{\mathbb{K}^{4}} d^{4} k \delta\left(\operatorname{kmod}\left(\Delta k_{\vec{k}}\right)\right) \sum_{\vec{\nu} \in \mathbb{B}^{4}} \Phi_{\vec{\nu}, \vec{k}} \hat{c}_{\uparrow \nu_{1}}^{\dagger}\left(k_{1}\right) \hat{c}_{\downarrow \nu_{2}}^{\dagger}\left(k_{2}\right) \hat{c}_{\downarrow \nu_{3}}\left(k_{3}\right) \hat{c}_{\uparrow \nu_{4}}\left(k_{4}\right) .
\end{aligned}
$$


In order to keep the notation short, we will not write the time dependence explicitly any more and we will put the function parameter in the index of functions and operators, i.e. $n_{\sigma \nu k}:=n_{\sigma \nu}(k, t)$. Throughout this calculation, we will enumerate the band indices by $\nu$ and $m$, and the corresponding momenta as $k$ and $q$, respectively. We will now start to calculate the Boltzmann equation for the momentum distribution $n_{\sigma \nu k}$. Thus, we need to know its change in time:

$$
\delta(0) \frac{\partial}{\partial t} n_{\sigma \nu k}=\frac{\partial}{\partial t}\left\langle\hat{n}_{\sigma \nu k}\right\rangle_{t}=\frac{i}{\hbar}\left\langle\left[\hat{n}_{\sigma \nu k}, \hat{H}\right]\right\rangle_{t} .
$$

The first part of $\left[\hat{n}_{\sigma \nu k}, \hat{H}\right]$ vanishes:

$$
\left[\hat{n}_{\sigma \nu k}, \hat{H}_{0}\right]=0
$$

Only the second part is left:

$$
\begin{aligned}
{\left[\hat{n}_{\sigma \nu k}, \hat{H}_{\mathrm{int}}\right]=} & \sum_{\vec{m}} \int d^{4} q \Phi_{\vec{m}, \vec{q}} \delta\left(\Delta k_{\vec{q}}\right) \hat{c}_{\uparrow m_{1} q_{1}}^{\dagger} \hat{c}_{\downarrow m_{2} q_{2}}^{\dagger} \hat{c}_{\downarrow m_{3} q_{3}} \hat{c}_{\uparrow m_{4} q_{4}} \\
& \times\left[\delta_{\uparrow \sigma} \delta_{m_{1} \nu} \delta_{q_{1} k}^{D}+\delta_{\downarrow \sigma} \delta_{m_{2} \nu} \delta_{q_{2} k}^{D}-\delta_{\downarrow \sigma} \delta_{m_{3} \nu} \delta_{q_{3} k}^{D}-\delta_{\uparrow \sigma} \delta_{m_{4} \nu} \delta_{q_{4} k}^{D}\right] \\
= & : U \hat{F}_{\sigma \nu k} .
\end{aligned}
$$

Eqs. (B.3) and (B.5) lead to

$$
i \hbar \delta(0) \frac{\partial}{\partial t} n_{\sigma \nu k}(t)=U\left\langle\hat{F}_{\sigma \nu k}\right\rangle_{t}
$$

Let us define the expectation value of the operator $\hat{F}_{\sigma \nu k}$ by

$$
F_{\sigma \nu k}(t):=\left\langle\hat{F}_{\sigma \nu k}\right\rangle_{t}
$$

Hence, the change of the momentum distribution can be written as

$$
i \hbar \delta(0) \frac{\partial}{\partial t} n_{\sigma \nu k}(t)=U F_{\sigma \nu k}(t)
$$

Now we need to find out how $F_{\sigma \nu k}(t)$ evolves in time. First, we define

$$
\hat{\varphi}_{\vec{m}, \vec{q}}:=\hat{c}_{\uparrow m_{1} q_{1}}^{\dagger} \hat{c}_{\downarrow m_{2} q_{2}}^{\dagger} \hat{c}_{\downarrow m_{3} q_{3}} \hat{c}_{\uparrow m_{4} q_{4}} \cdot
$$

This results in

$$
\begin{aligned}
F_{\sigma \nu k}(t)= & \sum_{\vec{m}} \int d^{4} q \Phi_{\vec{m}, \vec{q}} \delta\left(\Delta k_{\vec{q}}\right)\left\langle\hat{\varphi}_{\vec{m}, \vec{q}}\right\rangle_{t} \\
& \times\left[\delta_{\uparrow \sigma} \delta_{m_{1} n} \delta_{q_{1} k}^{D}+\delta_{\downarrow \sigma} \delta_{m_{2} n} \delta_{q_{2} k}^{D}-\delta_{\downarrow \sigma} \delta_{m_{3} n} \delta_{q_{3} k}^{D}-\delta_{\uparrow \sigma} \delta_{m_{4} n} \delta_{q_{4} k}^{D}\right]
\end{aligned}
$$

In order to calculate $F_{\sigma \nu k}(t)$, we need to know

$$
\varphi_{\vec{\nu}, \vec{k}}(t):=\left\langle\hat{\varphi}_{\vec{\nu}, \vec{k}}\right\rangle_{t} .
$$

Similar as for $n_{\sigma \nu k}(t)$, the change of the 4-point function is

$$
i \hbar \frac{\partial}{\partial t} \varphi_{\vec{\nu}, \vec{k}}(t)=\left\langle\left[\hat{H}, \hat{\varphi}_{\vec{\nu}, \vec{k}}\right]\right\rangle_{t} .
$$


The first part of the commutator is

$$
\left[\hat{H}_{0}, \hat{\varphi}_{\vec{\nu}, \vec{k}}\right]=\hbar \Delta \Omega_{\vec{\nu}, \vec{k}} \hat{\varphi}_{\vec{\nu}, \vec{k}},
$$

with $\Delta \Omega_{\vec{\nu}, \vec{k}}=\Omega_{\nu_{1}}\left(k_{1}\right)+\Omega_{\nu_{2}}\left(k_{2}\right)-\Omega_{\nu_{3}}\left(k_{3}\right)-\Omega_{\nu_{4}}\left(k_{4}\right)$.

$$
\Rightarrow\left(i \hbar \frac{\partial}{\partial t}+\hbar \Delta \Omega_{\vec{\nu}, \vec{k}}\right) \varphi_{\vec{\nu}, \vec{k}}(t)=U\left\langle\left[\hat{\Phi}, \hat{\varphi}_{\vec{\nu}, \vec{k}}\right]\right\rangle_{t}
$$

Its formal solution is

$$
\Rightarrow \varphi_{\vec{\nu}, \vec{k}}(t)=\varphi_{\vec{\nu}, \vec{k}}(0) e^{-i t \Delta \Omega_{\vec{\nu}, \vec{k}}}-\frac{i U}{\hbar} \int_{0}^{t} d s e^{-i(t-s) \Delta \Omega_{\vec{\nu}, \vec{k}}}\left\langle\left[\hat{\Phi}, \hat{\varphi}_{\vec{\nu}, \vec{k}}\right]\right\rangle_{s} .
$$

Plugging this into Eq. (B.10), we find

$$
\begin{aligned}
F_{\sigma \nu k}(t)= & \sum_{\vec{m}} \int d^{4} q \Phi_{\vec{m}, \vec{q}} \delta\left(\Delta k_{\vec{q}}\right) \\
& \times\left\{\varphi_{\vec{m}, \vec{q}}(0) e^{-i t \Delta \Omega_{\vec{m}, \vec{q}}}-\frac{i U}{\hbar} \int_{0}^{t} d s e^{\left.-i(t-s) \Delta \Omega_{\vec{m}, \vec{q}}\left\langle\left[\hat{\Phi}, \hat{\varphi}_{\vec{m}, \vec{q}}\right]\right\rangle_{s}\right\}}\right. \\
& \times\left[\delta_{\uparrow \sigma} \delta_{m_{1} \nu} \delta_{q_{1} k}^{D}+\delta_{\downarrow \sigma} \delta_{m_{2} \nu} \delta_{q_{2} k}^{D}-\delta_{\downarrow \sigma} \delta_{m_{3} \nu} \delta_{q_{3} k}^{D}-\delta_{\uparrow \sigma} \delta_{m_{4} \nu} \delta_{q_{4} k}^{D}\right] \\
= & F_{\sigma \nu k}^{(0)}(t)-\frac{i U}{\hbar} \int_{0}^{t} d s\left\langle\left[\hat{\Phi}, \hat{G}_{\sigma \nu k}(t-s)\right]\right\rangle_{s} .
\end{aligned}
$$

Here, we introduced

$$
\begin{aligned}
F_{\sigma \nu k}^{(0)}(t):= & \sum_{\vec{m}} \int d^{4} q \Phi_{\vec{m}, \vec{q}} \delta\left(\Delta k_{\vec{q}}\right) e^{-i t \Delta \Omega_{\vec{m}, \vec{q}}} \varphi_{\vec{m}, \vec{q}}(0) \\
& \times\left[\delta_{\uparrow \sigma} \delta_{m_{1} \nu} \delta_{q_{1} k}^{D}+\delta_{\downarrow \sigma} \delta_{m_{2} \nu} \delta_{q_{2} k}^{D}-\delta_{\downarrow \sigma} \delta_{m_{3} \nu} \delta_{q_{3} k}^{D}-\delta_{\uparrow \sigma} \delta_{m_{4} \nu} \delta_{q_{4} k}^{D}\right], \\
\hat{G}_{\sigma \nu k}(t):= & \sum_{\vec{m}} \int d^{4} q \Phi_{\vec{m}, \vec{q}} \delta\left(\Delta k_{\vec{q}}\right) e^{-i t \Delta \Omega_{\vec{m}, \vec{q}}} \hat{\varphi}_{\vec{m}, \vec{q}} \\
& \times\left[\delta_{\uparrow \sigma} \delta_{m_{1} \nu} \delta_{q_{1} k}^{D}+\delta_{\downarrow \sigma} \delta_{m_{2} \nu} \delta_{q_{2} k}^{D}-\delta_{\downarrow \sigma} \delta_{m_{3} \nu} \delta_{q_{3} k}^{D}-\delta_{\uparrow \sigma} \delta_{m_{4} \nu} \delta_{q_{4} k}^{D}\right] .
\end{aligned}
$$

Plugging Eq. (B.16) into Eq. (B.8), the change of the momentum distribution becomes

$$
\Rightarrow i \hbar \delta(0) \dot{n}_{\sigma \nu k}(t)=U F_{\sigma \nu k}^{(0)}(t)-\frac{i U^{2}}{\hbar} \int_{0}^{t} d s\left\langle\left[\hat{\Phi}, \hat{G}_{\sigma \nu k}(t-s)\right]\right\rangle_{s} .
$$

We introduce the following short notations for indices. First, we define $x:=$ $(\sigma \nu k)$. Next, $\vec{y}$ and $\vec{z}$ are supposed to be summing indices so that $y_{j}=$ $\left(\tau_{j}, m_{j}, q_{j}\right)$ and $z_{j}=\left(\tau_{j}, m_{j}^{\prime}, q_{j}^{\prime}\right)$, where $\vec{\tau}=(\uparrow, \downarrow, \downarrow, \uparrow)$ is fixed. Thus, we have

$$
\sum_{\vec{y}}[\ldots]=\sum_{\vec{m}} \int d^{4} q[\ldots]_{\vec{\sigma}=\vec{\tau}}
$$

Furthermore, if we use $\vec{y}$ as an index, sometimes only the relevant degrees of 
freedom are used, like in $\delta\left(\Delta k_{\vec{y}}\right)=\delta\left(\Delta k_{\vec{q}}\right)$ and $\Phi_{\vec{y}}=\Phi_{\vec{m}, \vec{q}}$.

$$
\begin{aligned}
{\left[\hat{\Phi}, \hat{G}_{\sigma \nu k}(t-s)\right] } & =\left[\hat{\Phi}, \hat{G}_{x}(t-s)\right] \\
& =-\sum_{\vec{y}, \vec{z}} \Phi_{\vec{y}} \delta\left(\Delta k_{\vec{y}}\right)\left[\hat{\varphi}_{\vec{y}}, \hat{\varphi}_{\vec{z}}\right] \Phi_{\vec{z}} \delta\left(\Delta k_{\vec{z}}\right) \delta_{\vec{z}}^{x} e^{-i(t-s) \Delta \Omega_{\vec{z}}} \\
& =\sum_{\vec{y}, \vec{z}} \hat{\varphi}_{\vec{y}} \hat{\varphi}_{\vec{z}}\left[\Phi_{\vec{y}} \delta\left(\Delta k_{\vec{y}}\right) G_{x, \vec{z}}(t-s)-\Phi_{\vec{z}} \delta\left(\Delta k_{\vec{z}}\right) G_{x, \vec{y}}(t-s)\right],
\end{aligned}
$$

where

$$
\begin{aligned}
& \delta_{\vec{y}}^{x}:=\delta_{y_{1} x}+\delta_{y_{2} x}-\delta_{y_{3} x}-\delta_{y_{4} x} \\
& \delta_{y_{j} x}:=\delta_{\tau_{j} \sigma} \delta_{m_{j} n} \delta_{q_{j} k}^{D} \\
& G_{x, \vec{y}}(t):=-\Phi_{\vec{y}} \delta\left(\Delta k_{\vec{y}}\right) e^{-i t \Delta \Omega_{\vec{y}}} \delta_{\vec{y}}^{x} \\
& M_{x}(\vec{y}, \vec{z}, t):=\Phi_{\vec{y}} \delta\left(\Delta k_{\vec{y}}\right) G_{x, \vec{z}}(t)-\Phi_{\vec{z}} \delta\left(\Delta k_{\vec{z}}\right) G_{x, \vec{y}}(t) \\
& \Rightarrow\left\langle\left[\hat{\Phi}, \hat{G}_{x}\left(t^{\prime}\right)\right]\right\rangle_{t}=\sum_{\vec{y}, \vec{z}}\left\langle\hat{\varphi}_{\vec{y}} \hat{\varphi}_{\vec{z}}\right\rangle_{t} M_{x}\left(\vec{y}, \vec{z}, t^{\prime}\right) .
\end{aligned}
$$

\section{B.1.2 Restricted quasi-freeness}

The next assumption we make use of is called restricted quasi-freeness. It means that the four- and eight-point functions fulfill a Wick theorem (see Eq. (23) of Erdös et al. (2004)), i.e.

$$
\left\langle\hat{\varphi}_{\vec{y}}\right\rangle_{t}=\left\langle\hat{c}_{y_{1}}^{\dagger} \hat{c}_{y_{2}}^{\dagger} \hat{c}_{y_{3}} \hat{c}_{y_{4}}\right\rangle_{t}=\left|\begin{array}{ll}
\nu_{y_{1} y_{4}} & \nu_{y_{1} y_{3}} \\
\nu_{y_{2} y_{4}} & \nu_{y_{2} y_{3}}
\end{array}\right|
$$

and

$$
\begin{aligned}
\left\langle\hat{\varphi}_{\vec{y}} \hat{\varphi}_{\vec{z}}\right\rangle_{t} & =\left\langle\hat{c}_{y_{1}}^{\dagger} \hat{c}_{y_{2}}^{\dagger} \hat{c}_{y_{3}} \hat{c}_{y_{4}} \hat{c}_{z_{1}}^{\dagger} \hat{c}_{z_{2}}^{\dagger} \hat{c}_{z_{3}} \hat{c}_{z_{4}}\right\rangle_{t} \\
& =\left|\begin{array}{cccc}
\nu_{y_{1} z_{4}} & \nu_{y_{1} z_{3}} & \nu_{y_{1} y_{4}} & \nu_{y_{1} y_{3}} \\
\nu_{y_{2} z_{4}} & \nu_{y_{2} z_{3}} & \nu_{y_{2} y_{4}} & \nu_{y_{2} y_{3}} \\
\nu_{z_{1} z_{4}} & \nu_{z_{1} z_{3}} & -\bar{\nu}_{z_{1} y_{4}} & -\bar{\nu}_{z_{1} y_{3}} \\
\nu_{z_{2} z_{4}} & \nu_{z_{2} z_{3}} & -\bar{\nu}_{z_{2} y_{4}} & -\bar{\nu}_{z_{2} y_{3}}
\end{array}\right|,
\end{aligned}
$$

with $\nu_{\alpha \beta}=\left\langle\hat{c}_{\alpha}^{\dagger} \hat{c}_{\beta}\right\rangle_{t}$ and $\bar{\nu}_{x y}:=\delta_{x y}-\nu_{x y}$.

The term "restricted quasi-freeness" is explained in appendix A in Erdös et al. (2004). As a first step, they define a quasi-free state $\hat{\rho}$ as a density matrix

$$
\hat{\rho}=\frac{1}{Z} e^{-\tilde{H}_{0}}
$$

Hereby, the hermitian operator $\tilde{H}_{0}$ has the form

$$
\tilde{H}_{0}=\sum_{y z} \hat{c}_{y}^{\dagger} Q_{y z} \hat{c}_{z}
$$

Erdös et al. (2004) show that such states fulfill Eq. (B.23) and Eq. (B.24). The restricted quasi-freeness does not necessarily assume a quasi-free state. It only assumes that Eq. (B.23) and Eq. (B.24) are fulfilled. 
From Eq. (B.23), it follows that

$$
\left\langle\hat{c}_{y_{1}}^{\dagger} \hat{c}_{y_{2}}^{\dagger} \hat{c}_{y_{3}} \hat{c}_{y_{4}}\right\rangle_{t}\left(\delta_{y_{1} x}^{D}+\delta_{y_{2} x}^{D}-\delta_{y_{3} x}^{D}-\delta_{y_{4} x}^{D}\right)=0 \quad \forall x .
$$

Proof:

- Case 1: $x \neq y_{j} \forall j$.

Eq. (B.27) is fulfilled because $\left(\delta_{y_{1} x}+\delta_{y_{2} x}-\delta_{y_{3} x}-\delta_{y_{4} x}\right)=0$.

- Case 2a: $x=y_{1}=y_{2}$ or $x=y_{3}=y_{4}$.

Eq. (B.27) is fulfilled because $\hat{c}_{x}^{\dagger} \hat{c}_{x}^{\dagger}=\hat{c}_{x} \hat{c}_{x}=0$.

- Case $x=y_{1}=y_{3}$ or $x=y_{1}=y_{4}$ or $x=y_{2}=y_{3}$ or $x=y_{2}=y_{4}$ :

W.l.o.g., we consider $x=y_{1}=y_{3}$. Then, the l.h.s. of Eq. (B.27) is reduced to $\left\langle\hat{c}_{x}^{\dagger} \hat{c}_{y_{2}}^{\dagger} \hat{c}_{x} \hat{c}_{y_{4}}\right\rangle_{t}\left(\delta_{y_{2} x}^{D}-\delta_{y_{4} x}^{D}\right)$. The second factor can only be non-zero if $y_{2} \neq y_{4}$, and if at least one of them is equal to $x$. Hence, either $\hat{c}_{x}^{\dagger} \hat{c}_{y_{2}}^{\dagger}=0$ or $\hat{c}_{x} \hat{c}_{y_{4}}=0$, and Eq. (B.27) is true.

- Case 3: $x$ is equal to exactly one of the $y_{j}$.

We assume w.l.o.g. that $x=y_{1}$. Then, we have $y_{1} \neq y_{3}$ and $y_{1} \neq y_{4}$, and therefore $\nu_{y_{1} y_{3}}=\nu_{y_{1} y_{4}}=0$.

All possible configurations of the $y_{j}$ fit in one of these cases. Thus, the proof is done.

The equality Eq. (B.27), which we just proved, leads to

$$
\begin{aligned}
& \varphi_{\vec{y}}(0)\left(\delta_{y_{1} x}^{D}+\delta_{y_{2} x}^{D}-\delta_{y_{3} x}^{D}-\delta_{y_{4} x}^{D}\right)=0 \\
& \Rightarrow F_{\sigma \nu k}^{(0)}(t)=0
\end{aligned}
$$

Therefore, the first term of the l.h.s of Eq. (B.18) drops out and we get

$$
\Rightarrow \delta(0) \frac{\partial}{\partial t} n_{\sigma \nu k}(t)=-\frac{U^{2}}{\hbar^{2}} \int_{0}^{t} d s\left\langle\left[\hat{\Phi}, \hat{G}_{\sigma \nu k}(t-s)\right]\right\rangle_{s} \cdot
$$

Using Eq. (B.1), which states that $\nu_{y z}=\delta_{y z} n_{y}(t)$, the 8-point function becomes

$$
\begin{aligned}
& \left\langle\hat{\varphi}_{\vec{y}} \hat{\varphi}_{z}\right\rangle_{t}=\left\langle\hat{c}_{y_{1}}^{\dagger} \hat{c}_{y_{2}}^{\dagger} \hat{c}_{y_{3}} \hat{c}_{y_{4}} \hat{c}_{z_{1}}^{\dagger} \hat{c}_{z_{2}}^{\dagger} \hat{c}_{z_{3}} \hat{c}_{z_{4}}\right\rangle_{t} \\
& =n_{y_{1}} n_{y_{2}}\left|\begin{array}{cccc}
\delta_{y_{1} z_{4}}^{D} & 0 & \delta_{y_{1} y_{4}}^{D} & 0 \\
0 & \delta_{y_{2} z_{3}}^{D} & 0 & \delta_{y_{2} y_{3}}^{D} \\
\delta_{z_{1} z_{4}}^{D} n_{z_{1}}(t) & 0 & -\delta_{z_{1} y_{4}}^{D} \bar{n}_{y_{4}}(t) & 0 \\
0 & \delta_{z_{2} z_{3}}^{D} n_{z_{2}}(t) & 0 & -\delta_{z_{2} y_{3}}^{D} \bar{n}_{y_{3}}(t)
\end{array}\right| \\
& =n_{y_{1}}(t) n_{y_{2}}(t)\left\{\delta_{y_{1} z_{4}}^{D}\left[\delta_{y_{2} z_{3}}^{D} \delta_{z_{1} y_{4}}^{D} \delta_{z_{2} y_{3}}^{D} \bar{n}_{y_{4}}(t) \bar{n}_{y_{3}}(t)+\delta_{y_{2} y_{3}}^{D} \delta_{z_{1} y_{4}}^{D} \delta_{z_{2} z_{3}}^{D} \bar{n}_{y_{4}}(t) n_{z_{2}}(t)\right]\right. \\
& \left.\quad+\delta_{y_{1} y_{4}}^{D}\left[\delta_{y_{2} y_{3}}^{D} \delta_{z_{1} z_{4}}^{D} \delta_{z_{2} z_{3}}^{D} n_{z_{1}}(t) n_{z_{2}}(t)+\delta_{y_{2} z_{3}}^{D} \delta_{z_{1} z_{4}}^{D} \delta_{z_{2} y_{3}}^{D} n_{z_{1}}(t) \bar{n}_{y_{3}}(t)\right]\right\} .
\end{aligned}
$$

Plugging this into Eq. (B.22), the first of the four term above gives

$$
\begin{aligned}
\rightarrow \sum_{\vec{y}} M_{x}\left(\vec{y} ; \vec{y}_{\mathrm{rev}} ; t^{\prime}\right) n_{y_{1}}(t) n_{y_{2}}(t) \bar{n}_{y_{3}}(t) \bar{n}_{y_{4}}(t)= \\
=\sum_{y_{1} y_{2} y_{4} z_{2}} \Phi_{\vec{y}} \Phi_{\vec{y}_{\mathrm{rev}}} n_{y_{1}}(t) n_{y_{2}}(t) \bar{n}_{y_{3}}(t) \bar{n}_{y_{4}}(t) \delta\left(\Delta k_{\vec{y}}\right) \\
\times \underbrace{\delta\left(\Delta k_{\left.\vec{y}_{\mathrm{rev}}\right)}\right)}_{=\delta\left(\Delta k_{\vec{y}}\right)}\left[\delta_{\vec{y}}^{x} e^{-i t^{\prime} \Delta \Omega_{\vec{y}}}-\delta_{\vec{y}_{\mathrm{rev}}}^{x} e^{-i t^{\prime} \Delta \Omega_{\vec{y}_{\mathrm{rev}}}}\right],
\end{aligned}
$$


with $\vec{y}_{\mathrm{rev}}=\left(y_{4}, y_{3}, y_{2}, y_{1}\right)$. The second terms is

$$
\begin{aligned}
& \rightarrow \sum_{y_{1} y_{2} y_{4} z_{2}} M_{x}\left(y_{1} y_{2} y_{2} y_{4} ; y_{4} z_{2} z_{2} y_{1} ; t^{\prime}\right) n_{y_{1}}(t) n_{y_{2}}(t) \bar{n}_{y_{4}}(t) n_{z_{2}}(t)= \\
&=\sum_{y_{1} y_{2} y_{4} z_{2}} \Phi_{y_{1} y_{2} y_{2} y_{4}} \Phi_{y_{4} z_{2} z_{2} y_{1}} n_{y_{1}}(t) n_{y_{2}}(t) \bar{n}_{y_{4}}(t) n_{z_{2}}(t) \underbrace{\delta\left(\Delta k_{y_{1} y_{2} y_{2} y_{4}}\right)}_{=\delta_{y_{1} y_{4}}^{D}} \\
& \quad \times \underbrace{\delta\left(\Delta k_{y_{4} z_{2} z_{2} y_{1}}\right)}_{=\delta_{y_{4} y_{1}}^{D}}[\underbrace{\delta_{y_{1} y_{2} y_{2} y_{4}}^{x}}_{=\delta_{y_{1} x}^{D}-\delta_{y_{4} x}^{D}} e^{-i t^{\prime} \Delta \Omega_{y_{1} y_{2} y_{2} y_{4}}}-\underbrace{\left.\delta_{y_{4} z_{2} z_{2} y_{1}}^{x} e^{-i t t^{\prime} \Delta \Omega_{y_{4} z_{2} z_{2} y_{1}}}\right]}_{=\delta_{y_{4} x}^{D}-\delta_{y_{1} x}^{D}} \\
&=0,
\end{aligned}
$$

where $e^{-i t \Delta \Omega_{\vec{y}}}=e^{-i t \Delta \Omega_{\vec{y}}}$. The third term gives

$$
\begin{aligned}
& \rightarrow \sum_{y_{1} y_{2} y_{4} z_{2}} M_{x}\left(y_{1} y_{2} y_{2} y_{1} ; z_{1} z_{2} z_{2} z_{1} ; t^{\prime}\right) n_{y_{1}}(t) n_{y_{2}}(t) n_{z_{1}}(t) n_{z_{2}}(t) \\
& =\sum_{y_{1} y_{2} y_{4} z_{2}} \Phi_{y_{1} y_{2} y_{2} y_{1}} \Phi_{z_{1} z_{2} z_{2} z_{1}} n_{y_{1}}(t) n_{y_{2}}(t) n_{z_{1}}(t) n_{z_{2}}(t) \delta\left(\Delta k_{y_{1} y_{2} y_{2} y_{1}}\right) \\
& \times \delta\left(\Delta k_{z_{1} z_{2} z_{2} z_{1}}\right)[\underbrace{\delta_{y_{1} y_{2} y_{2} y_{1}}^{x}}_{=0} e^{-i t^{\prime} \Delta \Omega_{y_{1} y_{2} y_{2} y_{1}}}-\underbrace{\delta_{z_{1} z_{2} z_{2} z_{1}}^{x}}_{=0} e^{-i t^{\prime} \Delta \Omega_{z_{1} z_{2} z_{2} z_{1}}}] \\
& =0 \text {. }
\end{aligned}
$$

The last term results in

$$
\begin{aligned}
\rightarrow \sum_{y_{1} y_{2} y_{4} z_{2}} & M_{x}\left(y_{1} y_{2} y_{2} y_{4} ; z_{1} z_{2} z_{3} z_{1} ; t^{\prime}\right) n_{y_{1}}(t) n_{y_{2}}(t) \bar{n}_{y_{3}}(t) n_{z_{1}}(t) \\
= & \sum_{y_{1} y_{2} y_{4} z_{2}} \Phi_{y_{1} y_{2} y_{2} y_{4}} \Phi_{z_{1} z_{2} z_{3} z_{1}} n_{y_{1}}(t) n_{y_{2}}(t) \bar{n}_{y_{3}}(t) n_{z_{1}}(t) \underbrace{\delta\left(\Delta k_{y_{1} y_{2} y_{2} y_{4}}\right)}_{=\delta_{y_{1} y_{4}}^{D}} \\
& \times \underbrace{\delta\left(\Delta k_{z_{1} z_{2} z_{3} z_{1}}\right)}_{=\delta_{z_{2} z_{3}}^{D}}[\underbrace{\delta_{y_{1} y_{2} y_{2} y_{4}}^{x}}_{=\delta_{y_{1} x}^{D}-\delta_{y_{4} x}^{D}} e^{-i t^{\prime} \Delta \Omega_{y_{1} y_{2} y_{2} y_{4}}}-\underbrace{\left.\delta_{z_{1} z_{2} z_{3} z_{2}}^{x} e^{-i t^{\prime} \Delta \Omega_{z_{1} z_{2} z_{3} z_{1}}}\right]}_{=\delta_{z_{2} x}^{D}-\delta_{z_{3} x}^{D}} \\
=0 . &
\end{aligned}
$$

Hence, only the first term does not vanish. Thus, we have

$$
\begin{aligned}
& \left\langle\left[\hat{\Phi}, \hat{G}_{x}\left(t^{\prime}\right)\right]\right\rangle_{t}= \\
& \quad=\sum_{\vec{y}}\left|\Phi_{\vec{y}}\right|^{2} \delta\left(\Delta k_{\vec{y}}\right) \delta(0)\left[\delta_{\vec{y}}^{x} e^{-i t^{\prime} \Delta \Omega_{\vec{y}}}-\delta_{\vec{y}_{\mathrm{rev}}}^{x} e^{-i t^{\prime} \Delta \Omega_{\vec{y}_{\mathrm{rev}}}}\right] n_{y_{1}}(t) n_{y_{2}}(t) \bar{n}_{y_{3}}(t) \bar{n}_{y_{4}}(t) .
\end{aligned}
$$

Here, we used the fact that $\Phi_{\vec{y}_{\text {rev }}}=\Phi_{\vec{y}}^{*}$. Now, we relabel the second term and define $\Delta n_{\vec{y}}(t):=n_{y_{1}}(t) n_{y_{2}}(t) \bar{n}_{y_{3}}(t) \bar{n}_{y_{4}}(t)-\bar{n}_{y_{1}}(t) \bar{n}_{y_{2}}(t) n_{y_{3}}(t) n_{y_{4}}(t)$ :

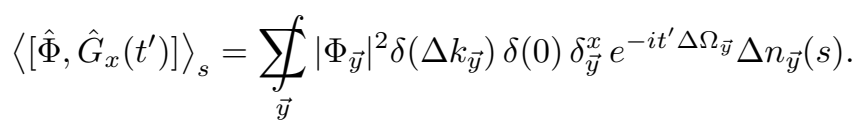


Plugging this into Eq. (B.29), we cancel the factor $\delta(0)$ and find

$$
\Rightarrow \frac{\partial}{\partial t} n_{x}(t)=-\frac{U^{2}}{\hbar^{2}} \sum_{\vec{y}}\left|\Phi_{\vec{y}}\right|^{2} \delta\left(\Delta k_{\vec{y}}\right) \delta_{\vec{y}}^{x} \int_{0}^{t} d s e^{-i(t-s) \Delta \Omega_{\vec{y}}} \Delta n_{\vec{y}}(s) .
$$

\section{B.1.3 Local approximation in time: A zero-interaction limit}

In the following, we show how the phase factor $e^{-i(t-s) \Delta \Omega_{\vec{y}}}$ becomes the energy conservation $\delta\left(\Delta \Omega_{\vec{y}}\right)$ by performing a certain $U \rightarrow 0$ limit. First, we define a non-zero (!) energy scale for the quadratic part of the Hamiltonian. We choose

$$
\hbar \zeta:=\max _{\nu, k} \Omega_{\nu}(k)
$$

because the only way it vanishes would be a trivial dispersion relation $\Omega_{\nu}(k) \equiv 0$. We use this to define dimensionless versions of the dispersion relation:

$$
\Delta \tilde{\Omega}:=\frac{\Delta \Omega}{\zeta} .
$$

The limit of low interaction means that the interaction term is much smaller than the other term in the Hamiltonian. Hence, we have to compare $U$ with $\hbar \zeta$ and define

$$
u:=\frac{U}{\hbar \zeta}
$$

Now, a dimensionless time $\tau$ is defined by

$$
\tau:=u^{2} \zeta t
$$

Here, we added the factor $u^{2}$, because we assume that we operate on times $t=\mathcal{O}\left(U^{-2}\right)$, so that

$$
\tau=\mathcal{O}(1)
$$

This way, we get the following dimensionless version of Eq. (B.37):

$$
\begin{aligned}
\frac{\partial}{\partial \tau} \bar{n}_{x}(\tau) & =\frac{1}{u^{2} \zeta} \frac{\partial}{\partial t} n_{x}(t) \\
& =-\frac{1}{u^{2} \zeta} u^{2} \zeta^{2} \sum_{\vec{y}}\left|\Phi_{\vec{y}}\right|^{2} \delta\left(\Delta k_{\vec{y}}\right) \delta_{\vec{y}}^{x} \int_{0}^{\tau} \frac{d \sigma}{u^{2} \zeta} e^{-\left(i / u^{2} \zeta\right)(\tau-\sigma) \zeta \Delta \tilde{\Omega}_{\vec{y}} \Delta \bar{n}_{\vec{y}}(\sigma)} \\
& =-\frac{1}{u^{2}} \sum_{\vec{y}}\left|\Phi_{\vec{y}}\right|^{2} \delta\left(\Delta k_{\vec{y}}\right) \delta_{\vec{y}}^{x} \int_{0}^{\tau} d \sigma e^{-i(\tau-\sigma) \Delta \tilde{\Omega}_{\vec{y}} / u^{2}} \Delta \bar{n}_{\vec{y}}(\sigma),
\end{aligned}
$$

where $\bar{n}_{y}(\tau):=n_{y}\left(\tau / U^{2} \zeta\right)$. Defining

$$
\begin{aligned}
\beta^{u}(E, x, \tau) & :=\sum_{\vec{y}}\left|\Phi_{\vec{y}}\right|^{2} \delta\left(\Delta k_{\vec{y}}\right) \delta_{\vec{y}}^{x} \frac{1}{u^{2}} e^{i(\tau-\sigma)\left(E-\Delta \tilde{\Omega}_{\vec{y}}\right) / u^{2}} \\
B(E, x, \tau): & =\lim _{u \rightarrow 0} \beta^{u}\left(E, x, \tau / u^{2}\right)
\end{aligned}
$$


we make use of the reasoning of Erdös et al. (2004), p. 377, and get

$$
\begin{aligned}
\frac{\partial}{\partial \tau} \bar{n}_{x}(\tau) & =-\pi B(0, x, \tau) \\
& =-\pi \sum_{\vec{y}}\left|\Phi_{\vec{y}}\right|^{2} \delta\left(\Delta k_{\vec{y}}\right) \delta_{\vec{y}}^{x} \delta\left(\Delta \tilde{\Omega}_{\vec{y}}\right) \Delta \bar{n}_{\vec{y}}(\tau)
\end{aligned}
$$

in leading order in $u$. At this point, the momentum distribution $n_{y}(t)$ has been replaced by the momentum distribution for $U=0$. Plugging the original dimensioned variables back into Eq. (B.45), one obtains

$$
\begin{aligned}
\frac{\partial}{\partial t} n_{x}(t) & =u^{2} \zeta \frac{\partial}{\partial \tau} \bar{n}_{x}(\tau) \\
& =-u^{2} \zeta \pi \sum_{\vec{y}}\left|\Phi_{\vec{y}}\right|^{2} \delta\left(\Delta k_{\vec{y}}\right) \delta_{\vec{y}}^{x} \delta\left(\Delta \Omega_{\vec{y}} / \zeta\right) \Delta n_{\vec{y}}(t) \\
& =-\frac{\pi U^{2}}{\hbar^{2}} \sum_{\vec{y}}\left|\Phi_{\vec{y}}\right|^{2} \delta\left(\Delta k_{\vec{y}}\right) \delta_{\vec{y}}^{x} \delta\left(\Delta \Omega_{\vec{y}}\right) \Delta n_{\vec{y}}(t) .
\end{aligned}
$$

Reducing $\delta_{\vec{y}}^{x}=\delta_{y_{1} x}^{D}+\delta_{y_{2} x}^{D}-\delta_{y_{3} x}^{D}-\delta_{y_{4} x}^{D}$, we gain a factor of two:

$$
\frac{\partial}{\partial t} n_{x}(t)=-\frac{2 \pi U^{2}}{\hbar^{2}} \sum_{\vec{y}}\left[\delta_{y_{1} x}^{D}+\delta_{y_{2} x}^{D}\right]\left|\Phi_{\vec{y}}\right|^{2} \delta\left(\Delta k_{\vec{y}}\right) \delta\left(\Delta \Omega_{\vec{y}}\right) \Delta n_{\vec{y}}(t) .
$$

Remember that $y_{j}=\left(\tau_{j}, n_{j}, k_{j}\right)$ and $\vec{\tau}=(\uparrow, \downarrow, \downarrow, \uparrow)$. Thus, if the multi-index $x$ is equal to $(\uparrow, n . k)$, the term with $\delta_{y_{2} x}^{D}$ vanishes and if it is $(\downarrow, n, k)$, the other term vanishes.

$$
\frac{\partial}{\partial t} n_{\uparrow, n_{1}, k_{1}}(t)=-\frac{2 \pi U^{2}}{\hbar^{2}} \int d k_{2} d k_{3} d k_{4} \delta\left(\Delta k_{\vec{k}}\right) \sum_{\nu_{2}, \nu_{3}, \nu_{4}} \delta\left(\Delta \Omega_{\vec{\nu}, \vec{k}}\right)\left|\Phi_{\vec{\nu}, \vec{k}}\right|^{2} \Delta n_{\vec{\tau}, \vec{\nu}, \vec{k}}(t),
$$

with $\Delta k_{\vec{k}}:=k_{1}+k_{2}-k_{3}-k_{4}$. Obviously, in $\Delta n_{\vec{\tau}, \vec{\nu}, \vec{k}}(t)$, both the $\uparrow-$ and the $\downarrow$-spin densities are involved. We assume a material with zero total spin and no magnetization, i.e. $n_{\uparrow n k}(t)=n_{\downarrow n k}(t)=: n_{\nu}(k, t)$.

$$
\frac{\partial}{\partial t} n_{\nu_{1}}\left(k_{1}, t\right)=-\frac{2 \pi U^{2}}{\hbar^{2}} \int d k_{2} d k_{3} d k_{4} \delta\left(\Delta k_{\vec{k}}\right) \sum_{\nu_{2}, \nu_{3}, \nu_{4}} \delta\left(\Delta \Omega_{\vec{\nu}, \vec{k}}\right)\left|\Phi_{\vec{\nu}, \vec{k}}\right|^{2} \Delta n_{\vec{\nu}}(\vec{k}, t),
$$

with $\vec{\nu}=\left(\nu_{1}, . ., \nu_{4}\right), \vec{k}=\left(k_{1}, \ldots, k_{4}\right)$ and

$$
\begin{aligned}
\Delta n_{\vec{\nu}}(\vec{k}, t):= & n_{\nu_{1}}\left(k_{1}, t\right) n_{\nu_{2}}\left(k_{2}, t\right)\left[1-n_{\nu_{3}}\left(k_{3}, t\right)\right]\left[1-n_{\nu_{4}}\left(k_{4}, t\right)\right] \\
& -\left[1-n_{\nu_{1}}\left(k_{1}, t\right)\right]\left[1-n_{\nu_{2}}\left(k_{2}, t\right)\right] n_{\nu_{3}}\left(k_{3}, t\right) n_{\nu_{4}}\left(k_{4}, t\right) .
\end{aligned}
$$

The limit $U \rightarrow 0$ was taken in such a way that the Boltzmann equation in Eq. (B.49) describes the time evolution of the quasiparticle momentum distribution. We make use of this Boltzmann equation in Eqs. (2.7), (3.9), and (4.50). 


\section{B.2 Linearization}

This section is establishing the scheme, with which we linearize the Boltzmann equation and obtain a linear rate equation. From App. B.1, we know

$$
\begin{aligned}
\dot{n}_{1}= & \mathcal{I}_{\text {coll }}[n]_{1} \\
\mathcal{I}_{\text {coll }}[n]_{1}:= & \frac{2 \pi U^{2}}{\hbar^{2}} \int_{\mathbb{K}^{3}} d k_{2} d k_{3} d k_{4} \delta\left(\Delta k_{\vec{k}}\right) \sum_{\substack{\nu_{2}, \nu_{3}, \nu_{4} \\
\in\{1, ., 4\}}} \delta\left(\Delta \Omega_{\vec{\nu}, \vec{k}}\right)\left|\Phi_{\vec{\nu}, \vec{k}}\right|^{2} \\
& \times\left[\left(1-n_{1}\right)\left(1-n_{2}\right) n_{3} n_{4}-n_{1} n_{2}\left(1-n_{3}\right)\left(1-n_{4}\right)\right] .
\end{aligned}
$$

We want to know the long time behavior of the quasiparticle momentum distribution. Therefore we linearize the quasiparticle momentum distribution and the Boltzmann equation around the thermal distribution, which is the Fermi-Dirac function in our case, i.e.

$$
f_{\nu}(k)=\frac{1}{\exp \left[\beta\left(\Omega_{\nu}-\mu\right)\right]+1} .
$$

The first step towards a linearized rate equation is defining a perturbation $\phi_{\nu}(k, t)$. We use the linearization scheme of Haug and Jauho (1996). The first step is defining the perturbation $\phi_{\nu}(k, t)$ of the quasiparticle momentum distribution by

$$
n_{\nu}(k, t)=\frac{1}{\exp \left[\beta\left(\Omega_{\nu}-\mu\right)-\phi_{\nu}(k)\right]+1} .
$$

The perturbation measures how far $n_{\nu}(k, t)$ is away from the thermal distribution. This can be seen by means of the expansion of $n_{\nu}(k, t)$ in $\phi_{\nu}(k)$ :

$$
n_{\nu}(k, t)=f_{\nu}(k)+f_{\nu}(k)\left[1-f_{\nu}(k)\right] \phi_{\nu}(k, t)+\mathcal{O}\left(\phi^{2}\right) .
$$

The time derivative applied to this equation yields

$$
\dot{n}_{\nu}(k, t)=f_{\nu}(k)\left[1-f_{\nu}(k)\right] \dot{\phi}_{\nu}(k, t)+\mathcal{O}\left(\phi^{2}\right) .
$$

Rearranging this equation leads to

$$
\dot{\phi}_{\nu}(k, t)=\frac{\dot{n}_{\nu}(k, t)}{f_{\nu}(k)\left[1-f_{\nu}(k)\right]}=\frac{\mathcal{I}_{\text {coll }}[n]_{\nu}(k, t)}{f_{\nu}(k)\left[1-f_{\nu}(k)\right]}=: \hat{\mathcal{L}}[\phi]_{\nu}(k, t)
$$

in first order in $\phi$. This defines the linear operator $\hat{\mathcal{L}}$, which maps the perturbation $\phi_{\nu}(k, t)$ to its change in time $\dot{\phi}_{\nu}(k, t)$. Introducing the notations $\bar{f}_{\nu}(k):=1-f_{\nu}(k)$ and $X_{j}=X_{\nu_{j}}\left(k_{j}, t\right)$ for every function $X$, we get

$$
\begin{aligned}
& \hat{\mathcal{L}}[\phi]_{1}=\underbrace{\frac{\mathcal{I}_{\text {coll }}[f]_{1}}{f_{1}\left(1-f_{1}\right)}}_{=0}-\frac{2 \pi U^{2}}{\hbar^{2} f_{1} \bar{f}_{1}} \int_{\mathbb{K}^{3}} d k_{2} d k_{3} d k_{4} \delta\left(\Delta k_{\vec{k}}\right) \sum_{\substack{\nu_{2}, \nu_{3}, \nu_{4} \\
\in\{1, \ldots, 4\}}} \delta\left(\Delta \Omega_{\vec{\nu}, \vec{k}}\right)\left|\Phi_{\vec{\nu}, \vec{k}}\right|^{2} \\
& \times\left[-f_{1} \bar{f}_{1} \phi_{1} f_{2} \bar{f}_{3} \bar{f}_{4}-f_{1} f_{2} \bar{f}_{2} \phi_{2} \bar{f}_{3} \bar{f}_{4}+f_{1} f_{2} \bar{f}_{3} f_{3} \phi_{3} \bar{f}_{4}+f_{1} f_{2} \bar{f}_{3} \bar{f}_{4} f_{4} \phi_{4}\right. \\
& \left.-\bar{f}_{1} f_{1} \phi_{1} \bar{f}_{2} f_{3} f_{4}-\bar{f}_{1} \bar{f}_{2} f_{2} \phi_{2} f_{3} f_{4}+\bar{f}_{1} \bar{f}_{2} f_{3} \bar{f}_{3} \phi_{3} f_{4}+\bar{f}_{1} \bar{f}_{2} f_{3} f_{4} \bar{f}_{4} \phi_{4}\right] \text {. }
\end{aligned}
$$


To do the next step, we consider

$$
\begin{aligned}
& f_{\nu_{1}}\left(k_{1}\right) f_{\nu_{2}}\left(k_{2}\right) \bar{f}_{\nu_{3}}\left(k_{3}\right) \bar{f}_{\nu_{4}}\left(k_{4}\right)= \\
& =\frac{1}{e^{\beta\left(\Omega_{1}(k)-\mu_{1}\right)}+1} \frac{1}{e^{\beta\left(\Omega_{2}(k)-\mu_{2}\right)}+1} \frac{1}{e^{-\beta\left(\Omega_{3}(k)-\mu_{3}\right)}+1} \frac{1}{e^{-\beta\left(\Omega_{4}(k)-\mu_{4}\right)}+1} \\
& =\bar{f}_{\nu_{1}}\left(k_{1}\right) \bar{f}_{\nu_{2}}\left(k_{2}\right) f_{\nu_{3}}\left(k_{3}\right) f_{\nu_{4}}\left(k_{4}\right) e^{-\beta \Delta \Omega_{\vec{\nu}, \vec{k}}+\beta(\mu+\mu-\mu-\mu)} .
\end{aligned}
$$

This is equivalent to

$$
f_{\nu_{1}}\left(k_{1}\right) f_{\nu_{2}}\left(k_{2}\right)\left[1-f_{\nu_{3}}\left(k_{3}\right)\right]\left[1-f_{\nu_{4}}\left(k_{4}\right)\right]-\left[1-f_{\nu_{1}}\left(k_{1}\right)\right]\left[1-f_{\nu_{2}}\left(k_{2}\right)\right] f_{\nu_{3}}\left(k_{3}\right) f_{\nu_{4}}\left(k_{4}\right)
$$

or in short notation

$$
f_{1} f_{2} \bar{f}_{3} \bar{f}_{4}=\bar{f}_{1} \bar{f}_{2} f_{3} f_{4} .
$$

We emphasize that this equation and the following derivation do not hold, if the final momentum distribution has different chemical potentials for each band. This may be the case, if the bands become too flat and particle exchange between bands is no longer possible. We assume a common chemical potential, i.e. $\mu_{n}=\mu$. If particle exchange is not possible, we will still see it in our results, because there will be additional eigenfunctions with zero eigenvalue, which are connected to change of local chemical potentials, see Eq. (4.66).

Eq. (B.59) leads to

$$
\begin{aligned}
\hat{\mathcal{L}}[\phi]_{1}= & \frac{2 \pi U^{2}}{\hbar^{2} f_{1} \bar{f}_{1}} \int_{\mathbb{K}^{3}} d k_{2} d k_{3} d k_{4} \delta\left(\Delta k_{\vec{k}}\right) \sum_{\substack{\nu_{2}, \nu_{3}, \nu_{4} \\
\in\{1, . ., 4\}}} \delta\left(\Delta \Omega_{\vec{\nu}, \vec{k}}\right)\left|\Phi_{\vec{\nu}, \vec{k}}\right|^{2} f_{1} f_{2} \bar{f}_{3} \bar{f}_{4} \\
& \times\left[\bar{f}_{1} \phi_{1}+\bar{f}_{2} \phi_{2}-f_{3} \phi_{3}-f_{4} \phi_{4}+f_{1} \phi_{1}+f_{2} \phi_{2}-\bar{f}_{3} \phi_{3}-\bar{f}_{4} \phi_{4}\right] .
\end{aligned}
$$

Finally, we obtain

$$
\begin{aligned}
\hat{\mathcal{L}}[\phi]_{1}= & \frac{2 \pi U^{2}}{\hbar^{2}} \int_{\mathbb{K}^{3}} d k_{2} d k_{3} d k_{4} \delta\left(\Delta k_{\vec{k}}\right) \sum_{\substack{\nu_{2}, \nu_{3}, \nu_{4} \\
\in\{1, \ldots, 4\}}} \delta\left(\Delta \Omega_{\vec{\nu}, \vec{k}}\right)\left|\Phi_{\vec{\nu}, \vec{k}}\right|^{2} \\
& \times \frac{\left(1-f_{2}\right) f_{3} f_{4}}{f_{1}}\left[\phi_{1}+\phi_{2}-\phi_{3}-\phi_{4}\right] .
\end{aligned}
$$

This linear operator is used for the linearized Boltzmann equations in Eqs. (2.21), (3.12), and (4.52).

\section{B.3 Scalar product, eigenfunctions, eigenvalues, and relaxation rates}

As in the previous section, we follow Haug and Jauho (1996) to find the scalar product and the relaxation rates. We define a scalar product and a corresponding norm using the thermal distribution $f_{\nu}(k)=1 /\left\{\exp \left[\beta\left(\hbar \Omega_{\nu}(k)-\mu\right)\right]+1\right\}$ :

$$
\begin{aligned}
\langle\phi, \psi\rangle_{\mathrm{F}} & :=\int d k \sum_{n} \phi_{\nu}(k) f_{\nu}(k)\left[1-f_{\nu}(k)\right] \psi_{\nu}(k), \\
\|\phi\|_{\mathrm{F}} & :=\sqrt{\langle\phi, \phi\rangle_{\mathrm{F}}} .
\end{aligned}
$$


$\hat{\mathcal{L}}$ is hermitian in this scalar product, i.e. :

$$
\langle\psi, \hat{\mathcal{L}}[\phi]\rangle_{\mathrm{F}}=\langle\hat{\mathcal{L}}[\psi], \phi\rangle_{\mathrm{F}} .
$$

Proof:

First, the 1.h.s. of Eq. (B.64) is considered:

$$
\begin{aligned}
\langle\psi, \hat{\mathcal{L}}[\phi]\rangle_{\mathrm{F}}= & \frac{2 \pi U^{2}}{\hbar^{2}} \int d^{4} k \delta\left(\Delta k_{\vec{k}}\right) \sum_{\vec{n}} \delta\left(\Delta \Omega_{\vec{\nu}, \vec{k}}\right) f_{\nu_{1}}\left(k_{1}\right) f_{\nu_{2}}\left(k_{2}\right) \bar{f}_{\nu_{3}}\left(k_{3}\right) \bar{f}_{\nu_{4}}\left(k_{4}\right) \\
& \times \psi_{\nu_{1}}\left(k_{1}\right)\left[\phi_{\nu_{1}}\left(k_{1}\right)+\phi_{\nu_{2}}\left(k_{2}\right)-\phi_{\nu_{3}}\left(k_{3}\right)-\phi_{\nu_{4}}\left(k_{4}\right)\right] .
\end{aligned}
$$

Using the short notation $X_{j}=X_{\nu_{j}}\left(k_{j}\right)$, we obtain

$$
\langle\psi, \hat{\mathcal{L}}[\phi]\rangle_{\mathrm{F}}=\frac{2 \pi U^{2}}{\hbar^{2}} \int d^{4} k \delta\left(\Delta k_{1234}\right) \sum_{\vec{n}} \delta\left(\Delta \Omega_{1234}\right) f_{1} f_{2} \bar{f}_{3} \bar{f}_{4} \psi_{1}\left[\phi_{1}+\phi_{2}-\phi_{3}-\phi_{4}\right] .
$$

Now, we are relabeling the momenta $k_{j}$ so that all $\phi_{j}$ are changed into $\phi_{1}$ :

$$
\begin{gathered}
\langle\psi, \hat{\mathcal{L}}[\phi]\rangle_{\mathrm{F}}=\frac{2 \pi U^{2}}{\hbar^{2}} \int d^{4} k \sum_{\vec{n}}\left[\delta\left(\Delta k_{1234}\right) \delta\left(\Delta \Omega_{1234}\right) f_{1} f_{2} \bar{f}_{3} \bar{f}_{4}\left(\psi_{1}+\psi_{2}\right)\right. \\
\left.-\delta\left(\Delta k_{3412}\right) \delta\left(\Delta \Omega_{3412}\right) f_{3} f_{4} \bar{f}_{1} \bar{f}_{2}\left(\psi_{3}+\psi_{4}\right)\right] \phi_{1} .
\end{gathered}
$$

Next, the identity $f_{1} f_{2} \bar{f}_{3} \bar{f}_{4}=\bar{f}_{1} \bar{f}_{2} f_{3} f_{4}$ is used, see Eq. (B.59). Moreover, we utilize the fact that $\Delta k_{3412}=\Delta k_{1234}$ and $\Delta \Omega_{3412}=\Delta \Omega_{1234}$ :

$$
\begin{aligned}
\langle\psi, \hat{\mathcal{L}}[\phi]\rangle_{\mathrm{F}} & =\frac{2 \pi U^{2}}{\hbar^{2}} \int d^{4} k \sum_{\vec{n}} \delta\left(\Delta k_{1234}\right) \delta\left(\Delta \Omega_{1234}\right) f_{1} f_{2} \bar{f}_{3} \bar{f}_{4}\left[\psi_{1}+\psi_{2}-\psi_{3}-\psi_{4}\right] \phi_{1} \\
& =\langle\hat{\mathcal{L}}[\psi], \phi\rangle_{\mathrm{F}} .
\end{aligned}
$$

We just proved that operator $\hat{\mathcal{L}}$ is hermitian. Therefore, its eigenfunctions $\chi_{\nu}^{(i)}(k)$ are orthogonal in the scalar product $\langle\cdot, \cdot\rangle_{\mathrm{F}} \cdot$

Proof:

$$
\begin{gathered}
\lambda_{i}\left\langle\chi^{(i)}, \chi^{(j)}\right\rangle_{\mathrm{F}}=\left\langle\hat{\mathcal{L}}\left[\chi^{(i)}\right], \chi^{(j)}\right\rangle_{\mathrm{F}}=\left\langle\chi^{(i)}, \hat{\mathcal{L}}\left[\chi^{(j)}\right]\right\rangle_{\mathrm{F}}=\lambda_{j}\left\langle\chi^{(i)}, \chi^{(j)}\right\rangle_{\mathrm{F}} \\
\Rightarrow\left(\lambda_{i}-\lambda_{j}\right)\left\langle\chi^{(i)}, \chi^{(j)}\right\rangle_{\mathrm{F}}=0 .
\end{gathered}
$$

If $\lambda_{i} \neq \lambda_{j}$, the claim is proven. Otherwise, we can orthogonalize the eigenfunctions, e.g. by the Gram-Schmidt procedure.

In essence, we find

$$
\left\langle\chi^{(i)}, \chi^{(j)}\right\rangle_{\mathrm{F}}=\left\|\chi^{(i)}\right\|_{\mathrm{F}}\left\|\chi^{(j)}\right\|_{\mathrm{F}} \delta_{i j}
$$

As a next step, we show that $\hat{\mathcal{L}}$ is positive semidefinite. 
Proof:

$$
\begin{aligned}
\langle\phi, \hat{\mathcal{L}}[\phi]\rangle_{\mathrm{F}}=\frac{2 \pi U^{2}}{\hbar^{2}} \int d^{4} k \sum_{\vec{n}} \delta\left(\Delta k_{1234}\right) \delta\left(\Delta \Omega_{1234}\right) f_{1} f_{2} \bar{f}_{3} \bar{f}_{4}\left[\phi_{1}+\phi_{2}-\phi_{3}-\phi_{4}\right] \phi_{1} \\
=\frac{2 \pi U^{2}}{\hbar^{2}} \int d^{4} k \sum_{\vec{n}} \delta\left(\Delta k_{1234}\right) \delta\left(\Delta \Omega_{1234}\right) f_{1} f_{2} \bar{f}_{3} \bar{f}_{4} \\
\quad \times\left[\phi_{1}+\phi_{2}-\phi_{3}-\phi_{4}\right] \frac{1}{4}\left[\phi_{1}+\phi_{1}+\phi_{1}+\phi_{1}\right] \\
=\frac{\pi U^{2}}{2 \hbar^{2}} \int d^{4} k \sum_{\vec{n}} \delta\left(\Delta k_{1234}\right) \delta\left(\Delta \Omega_{1234}\right) f_{1} f_{2} \bar{f}_{3} \bar{f}_{4} \\
\quad \times\left\{\left[\phi_{1}+\phi_{2}-\phi_{3}-\phi_{4}\right]\left[\phi_{1}+\phi_{2}\right]+\left[\phi_{3}+\phi_{4}-\phi_{1}-\phi_{2}\right]\left[\phi_{3}+\phi_{4}\right]\right\} \\
=\frac{\pi U^{2}}{2 \hbar^{2}} \int d^{4} k \sum_{\vec{n}} \delta\left(\Delta k_{1234}\right) \delta\left(\Delta \Omega_{1234}\right) \underbrace{f_{1} f_{2} \bar{f}_{3} \bar{f}_{4}}_{\geqslant 0} \underbrace{\left[\phi_{1}+\phi_{2}-\phi_{3}-\phi_{4}\right]^{2} \geqslant 0 .}_{\geqslant 0}
\end{aligned}
$$

Since $\hat{\mathcal{L}}$ is positive semidefinite, the eigenvalues $\lambda_{i}$ of $\hat{\mathcal{L}}$ are positive. Furthermore, they are the relaxation rates. To see this, we expand the perturbation $\phi_{\nu}(k, t)$ in the eigenfunctions $\chi_{\nu}^{(i)}(k)$ of $\hat{\mathcal{L}}$ :

$$
\phi_{\nu}(k, t)=\sum_{i} A_{i}(t) \chi_{\nu}^{(i)}(k)
$$

The time derivative of the r.h.s. is

$$
\sum_{i} \dot{A}_{i}(t) \chi_{\nu}^{(i)}(k)=\dot{\phi}_{\nu}(k, t) \stackrel{(B .62)}{=}-\sum_{i} A_{i}(t) \hat{\mathcal{L}}\left[\chi^{(i)}\right]_{\nu}(k)=-\sum_{i} A_{i}(t) \lambda_{i} \chi_{\nu}^{(i)}(k) .
$$

Hence, we have

$$
A_{j}(t)=A_{j}(0) e^{-\lambda_{j} t}
$$

Thus, one finds

$$
\phi_{\nu}(k, t)=\sum_{i} A_{i}(0) e^{-\lambda_{i} t} \chi_{\nu}^{(i)}(k)
$$

This shows that the eigenvalues $\lambda_{i}$ are the relaxation rates.

\section{B.4 Sommerfeld expansion of the scalar product}

The scalar product $\langle\cdot, \cdot\rangle_{\mathrm{F}}$ defined in Eq. (2.22) is important for the linearization of the Boltzmann equation. It can easily be calculated for small temperatures using a Sommerfeld expansion. The following calculation is for one band only 
for convenience.

$$
\begin{aligned}
\langle\phi, \psi\rangle_{\mathrm{F}} & =\int_{-1 / 2}^{1 / 2} d k \phi(k) f(k)[1-f(k)] \psi(k) \\
& =\int_{0}^{1 / 2} d k f(k)[1-f(k)][\phi(k) \psi(k)+\phi(-k) \psi(-k)] \\
& =-\int_{\omega(0)}^{\omega(1 / 2)} d \omega f(k(\omega))[1-f(k(\omega))] R[\phi, \psi](\omega) .
\end{aligned}
$$

Hereby, we defined

$$
R_{\phi \psi}(\omega):=k^{\prime}(\omega)[\phi(k(\omega)) \psi(k(\omega))+\phi(-k(\omega)) \psi(-k(\omega))]
$$

Assuming $R_{\phi \psi}(\omega)$ to be well behaved in the vicinity of $\omega=\mu$, we can replace it with its Taylor expansion around $\mu$.

$$
R_{\phi \psi}(\omega)=\sum_{n=0}^{\infty} \frac{1}{n !} R_{\phi \psi}^{(n)}(\mu)(\omega-\mu)^{n} .
$$

Plugging this into Eq. (B.77), we obtain

$$
\langle\phi, \psi\rangle_{\mathrm{F}}=\sum_{n=0}^{\infty} \frac{R_{\phi \psi}^{(n)}(\mu)}{(n) !} \int_{\omega(0)}^{\omega(1 / 2)} d \omega \frac{(\omega-\mu)^{n} e^{\beta(\omega-\mu)}}{\left(1+e^{\beta(\omega-\mu)}\right)^{2}}
$$

As long as $\mu$ is far enough away from zero or full filling, the integral over $\omega$ becomes

$$
\begin{aligned}
& \left(\frac{k_{\mathrm{B}} T}{2 J}\right)^{n} \int_{\beta(\omega(0)-\mu)}^{\beta(\omega(1 / 2)-\mu)} d x x^{n} \frac{e^{x}}{\left(1+e^{x}\right)^{2}} \\
& =\left(\frac{k_{\mathrm{B}} T}{2 J}\right)^{n} \int_{\mathbb{R}} d x x^{n} \frac{e^{x}}{\left(1+e^{x}\right)^{2}}+\mathcal{O}\left(e^{-\beta}\right) .
\end{aligned}
$$

The integral vanishes for an asymmetric integrand, i.e. for odd $n$. Thus, we have

$$
\langle\phi, \psi\rangle_{\mathrm{F}}=\sum_{n=0}^{\infty}\left(\frac{k_{\mathrm{B}} T}{2 J}\right)^{2 n+1} \frac{R_{\phi \psi}^{(2 n)}(\mu)}{(2 n) !} a_{n}+\mathcal{O}\left(e^{-\beta}\right)
$$

where $a_{n}=\int_{\mathbb{R}} d x x^{2 n} e^{x}\left(1+e^{x}\right)^{-2}$.

\section{B.5 N-point interpolation}

Here, we explain the interpolation of a function $g(k)$, while we only know the function values at the parameters $\kappa_{1}, \ldots, \kappa_{N_{\mathrm{k}}}$. At those points, $g(k)$ has values $g_{1}, \ldots, g_{N_{\mathrm{k}}}$, respectively. For the interpolation, we use exactly $N_{\mathrm{I}}$ of those points. That is similar to a spline interpolation or a local Lagrange interpolation, see for example Landau et al. (2007). 


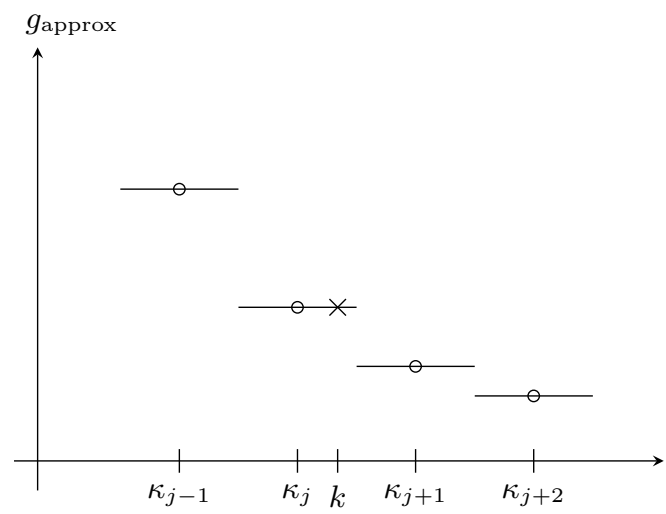

Figure B.1: Here, we see the 1-point-interpolation. It is an interpolation with a piecewise constant function. The local function $x \mapsto a=$ const is determined by one point $\kappa_{j}$ and its function value only. The advantage is that this is the fastest interpolation scheme in a numerical evaluation. The disadvantage is the lack of precision. This scheme can be used for a rough estimate.

Examples: For $N_{\mathrm{I}}=1$, one simply approximates $g(k)$ with $g_{j}$, where $j$ is such that $\left|k-\kappa_{j}\right|$ is minimal. This is depicted in Fig. B.1.

The case $N_{\mathrm{I}}=2$ is a linear interpolation, see Fig. B.2. We choose $j$ so that $\kappa_{j}<k<\kappa_{j+1}$ and so that our approximation becomes $g(k) \approx g_{j}$. One draws a line between the two points $\left(\kappa_{j}, g_{j}\right)$ and $\left(\kappa_{j+1}, g_{j+1}\right)$ with $\kappa_{j}<k<\kappa_{j+1}$. Thus, the interpolation reads

$$
g(k)=\frac{k-\kappa_{j+1}}{\kappa_{j}-\kappa_{j+1}} g_{j}+\frac{k-\kappa_{j}}{\kappa_{j+1}-\kappa_{j}} g_{j+1} .
$$

For $N_{\mathrm{I}}=3$, a parabola is used for interpolation. For larger $N_{\mathrm{I}}$, higher polynomials are used.

Arbitrary number of interpolation points $\mathrm{N}_{\mathrm{I}}$ : For the N-point interpolation of a function $g(k)$, we use those $N_{\mathrm{I}}$ discretization points $\kappa_{j}$ that lie closest to $k$. We denote the corresponding indices with $j_{1}, \ldots, j_{N_{\mathrm{I}}}$. The vector of discretization points, which are used for the interpolation, are denoted by

$$
\vec{v}(k):=\left(\kappa_{j_{1}}, \ldots, \kappa_{j_{N_{\mathrm{I}}}}\right) .
$$

At the beginning of this section, we already established that the function $g(k)$ is only known at the discretization points $\kappa_{j}$. Therefore, also

$$
\tilde{g}_{m}(k):=g\left(v_{m}(k)\right)
$$

is known for all $m=1, \ldots, N_{\mathrm{I}}$. The approximation of $g(k)$ is a linear combination of those known function values:

$$
g_{\text {approx }}(k):=\sum_{n=1}^{N_{\mathrm{I}}} \tilde{B}_{n}(k) \tilde{g}_{n}(k) .
$$




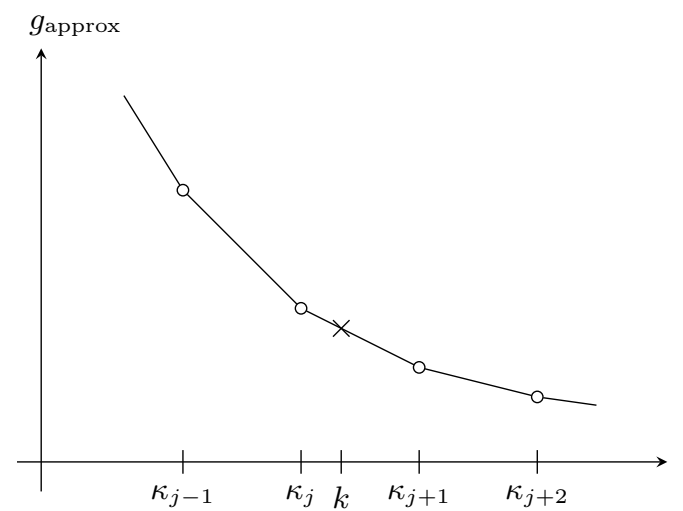

Figure B.2: This figure shows an example of a 2-point interpolation. It is an interpolation using a piecewise linear function. The local functions have the form $x \mapsto a+b x$. The two unknown parameters $a$ and $b$ are determined by the two points $\kappa_{j}$ and $\kappa_{j+1}$ and by their respective function values. We used this scheme for a first version of the numerical code for diagonalizing the linear Boltzmann operator for the single-band Hubbard model. Unfortunately, the precision of the eigenvalues was not high enough for our purposes.

The weights $\tilde{B}_{n}(k)$ are calculated in the following: The first step is a Taylor expansion of $g\left(v_{m}(k)\right)$ around $k$ :

$$
\tilde{g}_{m}(k)=\sum_{l=0}^{\infty} \frac{1}{l !} g^{(l)}\left(v_{m}(k)\right)\left(\kappa_{j}-v_{m}(k)\right)^{l}
$$

Plugging this into Eq. (B.86), we find

$$
\begin{aligned}
& g_{\text {approx }}(k)=\sum_{n=1}^{N_{\mathrm{I}}} \tilde{B}_{n}(k) \tilde{g}_{n}(k)=g(k) \underbrace{\sum_{n=1}^{N_{\mathrm{I}}} \tilde{B}_{n}(k)}_{! !} \\
& +\sum_{l=1}^{N_{\mathrm{I}}-1} \frac{1}{l !} g^{(l)}(k) \underbrace{\sum_{n=1}^{N_{\mathrm{I}}} \tilde{B}_{n}(k)\left(v_{n}(k)-k\right)^{l}}_{\stackrel{!}{=} 0} \\
& +\underbrace{\sum_{l=N_{\mathrm{I}}}^{\infty} \sum_{n=1}^{N_{\mathrm{I}}} \tilde{B}_{n}(k) \frac{1}{l !} g^{(l)}(k)\left(v_{n}(k)-k\right)^{l}}_{\text {error }} .
\end{aligned}
$$


This interpolation scheme assumes that the known points belong to a smooth function. The weights $\tilde{B}_{n}$ are determined by the $N$ equations

$$
\left[\begin{array}{ccc}
1 & \ldots & 1 \\
v_{1}(k)-k & \cdots & v_{N_{\mathrm{I}}}(k)-k \\
\vdots & & \vdots \\
\left(v_{1}(k)-k\right)^{N_{\mathrm{I}}-1} & \ldots & \left(v_{N_{\mathrm{I}}}(k)-k\right)^{N_{\mathrm{I}}-1}
\end{array}\right] \overrightarrow{\tilde{B}}(k)=\left[\begin{array}{c}
1 \\
0 \\
0 \\
\vdots \\
0
\end{array}\right] .
$$

This can be solved by thinking of what properties the $\tilde{B}_{n}$ should have. When $k$ is equal to one of the $v_{m}(k)$, then the weight of $g_{j_{m}}$ should be one and all others have to vanish. Therefore, it is reasonable to expect $\tilde{B}_{n}$ to be

$$
\tilde{B}_{n}(k)=\prod_{\substack{m=1 \\ m \neq n}}^{N_{\mathrm{I}}} \frac{k-v_{m}(k)}{v_{n}(k)-v_{m}(k)}
$$

This is local Lagrange interpolation. The discretized momenta are evenly distributed. Therefore, we can write $v_{n}(k)-v_{m}(k)=(n-m) / N_{\mathrm{k}}$. We use this to simplify Eq. (B.90):

$$
\tilde{B}_{n}(k)=\frac{(-1)^{n} N_{\mathrm{k}}^{N_{\mathrm{I}}-1}}{\left(N_{\mathrm{I}}-n\right) !(n-1) !} \prod_{\substack{m=1 \\ m \neq n}}^{N_{\mathrm{I}}}\left(k-v_{m}(k)\right) .
$$

Thus, the approximation of the function $g(k)$ is

$$
g_{\text {approx }}(k)=\sum_{n=1}^{N_{\mathrm{I}}} \tilde{g}_{n}(k) \frac{(-1)^{n} N_{\mathrm{k}}^{N_{\mathrm{I}}-1}}{\left(N_{\mathrm{I}}-n\right) !(n-1) !} \prod_{\substack{m=1 \\ m \neq n}}^{N_{\mathrm{I}}}\left(k-v_{m}(k)\right)
$$

At last, we define the coefficients $B_{j}(k)$ :

$$
B_{j}(k)= \begin{cases}\tilde{B}_{m}(k) & \text { for } j=j_{m} \\ 0 & \text { else. }\end{cases}
$$

Hence, we can write the approximation of $g(k)$ as

$$
g_{\text {approx }}(k):=\sum_{j=1}^{N_{\mathrm{k}}} B_{j}(k) g_{j}
$$

That form is more useful for calculating the discretized version of the linearized Boltzmann operator $\hat{\mathcal{L}}$ in Eq. (3.96).

Error estimation: We estimate the error by calculating a bound on the first error term. For the sake of simplicity, we only consider an odd number of discretization points $N_{\mathrm{I}}$. 
In order to determine the $v_{m}$, we have chosen those function parameters that lie closest to $k$. The $v_{m}$ have a distance $1 / N_{\mathrm{k}}$. Therefore, one finds

$$
\frac{N_{\mathrm{I}}}{2 N_{\mathrm{k}}}-\frac{m}{N_{\mathrm{k}}} \leqslant k-v_{m} \leqslant \frac{N_{\mathrm{I}}}{2 N_{\mathrm{k}}}-\frac{m}{N_{\mathrm{k}}}+\frac{1}{N_{\mathrm{k}}} .
$$

Moreover, we have

$$
\left|\left(k-v_{m}\right)\left(k-v_{N_{\mathrm{I}}+1-m}\right)\right| \leqslant\left|\frac{N_{\mathrm{I}}+1}{N_{\mathrm{k}}}-\frac{m}{N_{\mathrm{k}}}\right|^{2} .
$$

Thus, for $n \neq \frac{N_{\mathrm{I}}+1}{2}$ we find the bounds

$$
\prod_{\substack{m=1 \\ m \neq n}}^{N_{\mathrm{I}}}\left(k-v_{m}\right) \leqslant \begin{cases}\frac{1}{N_{\mathrm{k}}^{N_{\mathrm{I}}-1}}\left[\frac{N_{\mathrm{I}}-1}{2} !\right]^{2} \frac{\left|N_{\mathrm{I}}-2 n\right|}{\left(N_{\mathrm{I}}+1-2 n\right)^{2}} & \text { for } n \neq \frac{N_{\mathrm{I}}+1}{2} \\ \frac{1}{N_{\mathrm{k}}^{N_{\mathrm{I}}-1}}\left[\frac{N_{\mathrm{I}}-1}{2} !\right]^{2} & \text { for } n=\frac{N_{\mathrm{I}}+1}{2} .\end{cases}
$$

These give bounds for the weights:

$$
\begin{aligned}
\left|\tilde{B}_{n}(k)\right| & \leqslant \begin{cases}\left(\begin{array}{c}
N_{\mathrm{I}}-1 \\
n-1
\end{array}\right) \frac{1}{\left(N_{\mathrm{I}}-1\right) !}\left[\frac{N_{\mathrm{I}}-1}{2} !\right]^{2} \frac{\left|N_{\mathrm{I}}-2 n\right|}{\left(N_{\mathrm{I}}+1-2 n\right)^{2}} \leqslant \frac{1}{4} & \text { for } n \neq \frac{N_{\mathrm{I}}+1}{2} \\
\left(\begin{array}{c}
N_{\mathrm{I}}-1 \\
\left(N_{\mathrm{I}}-1\right) / 2
\end{array}\right) \frac{1}{\left(N_{\mathrm{I}}-1\right) !}\left[\frac{N_{\mathrm{I}}-1}{2} !\right]^{2}=1 & \text { for } n=\frac{N_{\mathrm{I}}+1}{2}\end{cases} \\
& \leqslant 1 .
\end{aligned}
$$

Thence, the error term can be bound by

$$
\begin{aligned}
& \left|\tilde{B}_{n}(k) \frac{1}{N_{\mathrm{I}} !} g^{\left(N_{\mathrm{I}}\right)}(k)\left(k-v_{n}\right)^{N_{\mathrm{I}}}\right| \\
& \leqslant \frac{\left\|g^{\left(N_{\mathrm{I}}\right)}\right\|_{\infty}}{N_{\mathrm{I}} !}\{\sum_{n=1}^{\left(N_{\mathrm{I}}-1\right) / 2}\left|\tilde{B}_{n}(k)\right| \underbrace{\left(k-v_{n}\right.}_{\leqslant\left(N_{\mathrm{I}}+1-2 n\right) / 2 L})^{N_{\mathrm{I}}}+\left|\tilde{B}_{N_{\mathrm{I}}+1-n}(k)\right| \underbrace{v_{N_{\mathrm{I}}+1-n}-k}_{\leqslant\left(N_{\mathrm{I}}+1-2 n\right) / 2 L})^{N_{\mathrm{I}}} \\
& \left.+\left|\tilde{B}_{\left(N_{\mathrm{I}}+1\right) / 2}(k)\right||\underbrace{\mid k-v_{\left(N_{\mathrm{I}}+1\right) / 2}}_{\leqslant 1 / 2 L}|^{N_{\mathrm{I}}}\right\} \\
& \leqslant \frac{\left\|g^{\left(N_{\mathrm{I}}\right)}\right\|_{\infty}}{(2 L)^{N_{\mathrm{I}}} N_{\mathrm{I}} !}\left\{2 \sum_{n=1}^{\left(N_{\mathrm{I}}-1\right) / 2}\left(\begin{array}{c}
N_{\mathrm{I}}-1 \\
n-1
\end{array}\right)\left(\begin{array}{c}
N_{\mathrm{I}}-1 \\
\left(N_{\mathrm{I}}-1\right) / 2
\end{array}\right)^{-1}+1\right\} \\
& =\frac{1}{N_{\mathrm{k}}^{N_{\mathrm{I}}}} \frac{\left\|g^{\left(N_{\mathrm{I}}\right)}\right\|_{\infty}}{2 N_{\mathrm{I}} !\left(\begin{array}{c}
N_{\mathrm{I}}-1 \\
\left(N_{\mathrm{I}}-1\right) / 2
\end{array}\right)} .
\end{aligned}
$$

Even for functions with a relatively large $N_{\mathrm{I}}^{\text {th }}$ derivative, the error is still small, because it is suppressed by $N_{\mathrm{k}}^{-N_{\mathrm{I}}}$. For instance, with $N_{\mathrm{I}}=9$ and $N_{\mathrm{k}}=2000$, this factor becomes $2 \cdot 10^{-30}$, which is extremely small. 


\section{Appendix C}

\section{Calculations for the Hubbard model}

\section{C.1 Band form of the Hubbard Hamiltonian}

In this section, we will show the textbook transformation of the Hubbard Hamiltonian in Eq. (3.3) to its 1-band form in Eq. (3.4). First, we define the transformed creation and annihilation operators:

$$
\begin{aligned}
& \hat{c}_{\sigma}(k)=\sum_{l} e^{-i 2 \pi k j} \hat{c}_{l, \sigma} \\
& \hat{c}_{\sigma}^{\dagger}(k)=\sum_{l} e^{i 2 \pi k j} \hat{c}_{l, \sigma}^{\dagger} .
\end{aligned}
$$

Due to $\delta_{l, 0}=\int d k e^{i 2 \pi k l}$, the back-transforms are

$$
\begin{gathered}
\hat{c}_{l, \sigma}=\int_{-1 / 2}^{1 / 2} d k e^{i 2 \pi k j} \hat{c}_{\sigma}(k) \\
\hat{c}_{l, \sigma}^{\dagger}=\int_{-1 / 2}^{1 / 2} d k e^{-i 2 \pi k j} \hat{c}_{\sigma}^{\dagger}(k) .
\end{gathered}
$$


Using these transformations, we can perform the transformation of the hopping part:

$$
\begin{aligned}
\hat{H}_{0} & =\sum_{l \in \mathbb{Z}} \sum_{\sigma \in\{\uparrow, \downarrow\}}\left[-J \hat{c}_{l, \sigma}^{\dagger} \hat{c}_{l+1, \sigma}-J^{\prime} \hat{c}_{l, \sigma}^{\dagger} \hat{c}_{l+2, \sigma}\right]+H . c . \\
& =-\sum_{l \sigma} \int d k d k^{\prime} \hat{c}_{\sigma}^{\dagger}(k) \hat{c}_{\sigma}\left(k^{\prime}\right)\left[\left(J e^{-i 2 \pi\left(k l-k^{\prime}(l+1)\right)}+J^{\prime} e^{-i 2 \pi\left(k l-k^{\prime}(l+2)\right)}\right)+c . c .\right] \\
& =-\sum_{\sigma} \int d k d k^{\prime} \hat{c}_{\sigma}^{\dagger}(k) \hat{c}_{\sigma}\left(k^{\prime}\right) \delta\left(k-k^{\prime}\right)\left[\left(J e^{-i 2 \pi k}+J^{\prime} e^{-i 4 \pi k}\right)+c . c .\right] \\
& =-\sum_{\sigma} \int d k \hat{c}_{\sigma}^{\dagger}(k) \hat{c}_{\sigma}(k)\left[2 J \cos (2 \pi k)+2 J^{\prime} \cos (4 \pi k)\right] .
\end{aligned}
$$

With the number operator $\hat{n}_{\sigma}(k)=\hat{c}_{\sigma}^{\dagger}(k) \hat{c}_{\sigma}(k)$, the dimensionless dispersion relation $\Omega_{\nu}(k)=\cos (2 \pi k)+\varepsilon \cos (4 \pi k)$, and the dimensionless next-to-nearestneighbor hopping $\varepsilon=J^{\prime} / J$, the last line resembles the 1-band form of $\hat{H}_{0}$ in Eq. (3.4). The interaction part of the Hamiltonian transforms in the following way:

$$
\begin{aligned}
\hat{H}_{\mathrm{int}} & =U \sum_{l \in \mathbb{Z}} \hat{n}_{l, \uparrow} \hat{n}_{l, \downarrow}=U \sum_{l \in \mathbb{Z}} \hat{c}_{l, \uparrow}^{\dagger} \hat{c}_{l, \uparrow} \hat{c}_{l, \downarrow}^{\dagger} \hat{c}_{l, \downarrow}=U \sum_{l \in \mathbb{Z}} \hat{c}_{l, \uparrow}^{\dagger} \hat{c}_{l, \downarrow}^{\dagger} \hat{c}_{l, \downarrow} \hat{c}_{l, \uparrow} \\
& =U \sum_{l} \int d^{4} k \hat{c}_{\uparrow}^{\dagger}\left(k_{1}\right) \hat{c}_{\downarrow}^{\dagger}\left(k_{2}\right) \hat{c}_{\downarrow}\left(k_{3}\right) \hat{c}_{\uparrow}\left(k_{4}\right) e^{-i 2 \pi\left(k_{1} l+k_{2} l-k_{3} l-k_{4} l\right)} \\
& =U \int d^{4} k \delta\left(\operatorname{kmod}\left(k_{1}+k_{2}-k_{3}-k_{4}\right)\right) \hat{c}_{\uparrow}^{\dagger}\left(k_{1}\right) \hat{c}_{\downarrow}^{\dagger}\left(k_{2}\right) \hat{c}_{\downarrow}\left(k_{3}\right) \hat{c}_{\uparrow}\left(k_{4}\right) .
\end{aligned}
$$

This is the interaction Hamiltonian's 1-band form in Eq. (3.4). The kmod function is defined in Eq. (2.3). It comes from the 1-periodicity of the function $k \mapsto \exp (i 2 \pi l k)$ for any $l \in \mathbb{Z}$.

\section{C.2 Monotonicity of the NNN dispersion relation}

The monotonicity of the dispersion relation is important for finding a numerical scheme. This numerical scheme uses a certain expression for the solution of the energy conservation, see Sec. 3.2.3.

The extrema of the dispersion relation are given by

$$
\begin{gathered}
0=\omega^{\prime}(k)=2 \pi \sin (2 \pi k)+4 \pi \varepsilon \sin (4 \pi k)=2 \pi \sin (2 \pi k)[1+4 \varepsilon \cos (2 \pi k)] \\
\Longleftrightarrow \sin (2 \pi k)=0 \vee \cos (2 \pi k)=-\frac{1}{4 \varepsilon} .
\end{gathered}
$$

For $\varepsilon<\frac{1}{4}$, the cosine equation has no solutions. In this case, the only extrema within the Brillouin zone are at $k=0, \pm \frac{1}{2}$. With $k=0$ being a minimum, $\omega(k)$ is monotonically increasing in the region $k \in\left(0, \frac{1}{2}\right)$ and monotonically decreasing for $k \in\left(-\frac{1}{2}, 0\right)$. 


\section{C.3 The inverse dispersion relation and its first two derivatives}

In this section, we calculate the inverse dispersion relation and its first two derivatives, which is used in Apps. C.4 and C.6.1. First, we calculate the inverse $k(\omega)$ of the dispersion relation $k \rightarrow \omega(k)$ with $\left[0, \frac{1}{2}\right] \mapsto \mathbb{R}$ :

$$
\begin{aligned}
& \omega(k)=\omega \\
& \Rightarrow 2 \varepsilon c_{k}^{2}+c_{k}-\varepsilon+\omega=0 \\
& \Rightarrow c_{k}=\frac{-1+\sqrt{1-8 \varepsilon(\omega-\varepsilon)}}{4 \varepsilon}
\end{aligned}
$$

The plus sign solution is not possible for the $\varepsilon$ we chose. We find

$$
\begin{aligned}
k(\omega) & =\arccos \left(\frac{\sqrt{1-8 \varepsilon(\omega-\varepsilon)}-1}{4 \varepsilon}\right) \\
& =\frac{1}{2 \pi} \arccos (\varepsilon-\omega)+\frac{\varepsilon(\omega-\varepsilon)^{2}}{\pi \sqrt{1-(\omega-\varepsilon)^{2}}}+\mathcal{O}\left(\varepsilon^{2}\right) \\
& =\frac{1}{4}+\frac{1}{2 \pi} \arcsin (\omega-\varepsilon)+\frac{\varepsilon(\omega-\varepsilon)^{2}}{\pi \sqrt{1-(\omega-\varepsilon)^{2}}}+\mathcal{O}\left(\varepsilon^{2}\right) .
\end{aligned}
$$

The first derivative has the form

$$
\begin{aligned}
k^{\prime}(\omega) & =\frac{\varepsilon}{\pi} \sqrt{\frac{2}{[1-8 \varepsilon(\omega-\varepsilon)][\sqrt{1-8 \varepsilon(\omega-\varepsilon)}-1+4 \varepsilon(\omega+\varepsilon)]}} \\
& =\frac{1}{2 \pi \sqrt{1-(\omega-\varepsilon)^{2}}}+\frac{\varepsilon(\omega-\varepsilon)\left[2-(\omega-\varepsilon)^{2}\right]}{\pi\left[1-(\omega-\varepsilon)^{2}\right]^{3 / 2}}+\mathcal{O}\left(\varepsilon^{2}\right) .
\end{aligned}
$$

The second derivative is

$$
k^{\prime \prime}(\omega)=\frac{\omega-\varepsilon}{2 \pi \sqrt{1-(\omega-\varepsilon)^{3 / 2}}}+\frac{\varepsilon\left[2+(\omega-\varepsilon)^{2}\right]}{\pi\left[1-(\omega-\varepsilon)^{2}\right]^{5 / 2}}+\mathcal{O}\left(\varepsilon^{2}\right) .
$$

The example seems to be quite generic, because it exhibits the the same features, which were already discovered for the calculation for general $\phi_{0}(k)$.

We can express the derivatives of $\omega\left(k_{\mathrm{F}}\right)$ by the derivatives of $k(\mu)$. For this, we use $\omega^{\prime}\left(k_{\mathrm{F}}\right) k_{\mathrm{F}}^{\prime} \equiv \omega^{\prime}(k(\mu)) k^{\prime}(\mu)=(d / d \mu) \omega(k(\mu))=1$. Thus, we have

$$
\begin{aligned}
\omega^{\prime}\left(k_{\mathrm{F}}\right) & =\frac{1}{k_{\mathrm{F}}^{\prime}} \\
\omega^{\prime \prime}\left(k_{\mathrm{F}}\right) & =-\frac{k_{\mathrm{F}}^{\prime \prime}}{\left(k_{\mathrm{F}}^{\prime}\right)^{2}} .
\end{aligned}
$$

\section{C.4 Small-temperature behavior of certain scalar products}

In this section, we perform the calculations of a certain scalar product of the form $\left\langle\phi, \chi^{(3)}\right\rangle_{\mathrm{F}}$, which are needed for Sec. 3.5.3. In order to perform these 
calculations, we make use the Sommerfeld expansion in App. B.4. Furthermore, we will replace the eigenfunction $\chi^{(3)}(k)$ with its approximation $\tilde{\chi}^{(3)}(k)=k-$ $\frac{1}{4} \operatorname{sgn}(k)$. This approximation is justified in Sec. 3.5.1. Moreover, we denote $k_{\mathrm{F}}^{\prime}:=k^{\prime}(\mu), k_{\mathrm{F}}^{\prime \prime}:=k^{\prime \prime}(\mu)$, and $k_{\mathrm{F}}^{\prime \prime \prime}:=k^{\prime \prime \prime}(\mu)$.

First, we calculate the scalar product of the third eigenfunction and the particle current's function $j^{N}(k)=\Omega^{\prime}(k)$, see Eq. (2.38):

$$
\begin{aligned}
\left\langle\tilde{\chi}^{(3)}, j^{N}\right\rangle_{\mathrm{F}} & =\sum_{n=0}^{\infty}\left(\frac{k_{\mathrm{B}} T}{2 J}\right)^{2 n+1} \frac{1}{(2 n) !} a_{n} \frac{d^{2 n}}{d \mu^{2 n}}\left[k_{\mathrm{F}}-\frac{1}{4} \operatorname{sgn}\left(k_{\mathrm{F}}\right)\right] \\
& =\frac{a_{0} k_{\mathrm{B}} T}{J}\left[k_{\mathrm{F}}-\frac{1}{4}\right]+\frac{a_{1} k_{\mathrm{B}}^{3}}{16 J^{3}} k_{\mathrm{F}}^{\prime \prime} T^{3}+\mathcal{O}\left(T^{5}\right) .
\end{aligned}
$$

Note that the derivative of the sign function is zero, as long as $k_{\mathrm{F}}$ is not near the band edges. With the help of the formulas for $k_{\mathrm{F}}$ and $k_{\mathrm{F}}^{\prime \prime}$ in App. C.3, we calculate

$$
\begin{aligned}
&\left\langle\tilde{\chi}^{(3)}, j^{N}\right\rangle_{\mathrm{F}}=\left\{\begin{array}{lr}
\frac{a_{0} k_{\mathrm{B}}}{2 J}\left[k_{\mathrm{F}}-\frac{1}{4}\right] T+\mathcal{O}\left(T^{3}\right) & \text { for } \varepsilon\left(k_{\mathrm{B}} T\right)^{2} \ll|\mu-\varepsilon| \\
\frac{a_{1} k_{\mathrm{B}}^{3}}{16 J^{3}} k_{\mathrm{F}}^{\prime \prime} T^{3}+\mathcal{O}\left(T^{5}\right) & \text { for }|\mu-\varepsilon| \ll \varepsilon\left(k_{\mathrm{B}} T\right)^{2}
\end{array}\right. \\
&=\left\{\begin{array}{lr}
\frac{a_{0} k_{\mathrm{B}}}{4 \pi J} \arcsin (\mu-\varepsilon) T+\mathcal{O}\left(\varepsilon(\mu-\varepsilon)^{2} T\right)+\mathcal{O}\left(T^{3}\right) \\
\text { for } \varepsilon\left(k_{\mathrm{B}} T\right)^{2} \ll|\mu-\varepsilon| \\
\frac{a_{1} k_{\mathrm{B}}^{3}}{8 \pi J^{3}} \varepsilon T^{3}+\mathcal{O}\left(\varepsilon^{2} T^{3}\right)+\mathcal{O}\left(T^{5}\right) & \text { for }|\mu-\varepsilon| \ll \varepsilon\left(k_{\mathrm{B}} T\right)^{2} .
\end{array}\right.
\end{aligned}
$$

Obviously, this scalar product has a different leading term in different situations. This is used in Eq. (3.129).

Next, we consider the scalar product with the heat current's function $j^{Q}=$ $j^{E}-\mu j^{N}$, where $j^{E}(k)=\Omega^{\prime}(k) \omega(k)$ is the function of the total kinetic energy current, see Eq. (2.38). Using the Sommerfeld expansion in Eq. (B.82), we find

$$
\begin{aligned}
\left\langle\chi^{(3)}, j^{Q}\right\rangle_{\mathrm{F}}= & \sum_{n=0}^{\infty}\left(\frac{k_{\mathrm{B}} T}{2 J}\right)^{2 n+1} \frac{a_{n}}{(2 n) !} \frac{d^{2 n}}{d \tilde{\omega}^{2 n}} 2 k^{\prime}(\tilde{\omega})\left[k(\tilde{\omega})-\frac{1}{4} \operatorname{sgn}(k(\tilde{\omega}))\right] \\
& \times\left.\omega^{\prime}(k(\tilde{\omega}))[\omega(k(\tilde{\omega}))-\mu]\right|_{\tilde{\omega}=\mu}+\mathcal{O}\left(e^{-\beta}\right) .
\end{aligned}
$$

With $\omega^{\prime}(k(\tilde{\omega})) k^{\prime}(\tilde{\omega})$ being equal to one and all the derivatives of $\operatorname{sgn}(k)$ vanishing at $k=k_{\mathrm{F}}$, we obtain

$$
\begin{aligned}
\left\langle\chi^{(3)},\right. & \left.j^{Q}\right\rangle_{\mathrm{F}}=\left.\sum_{n=0}^{\infty}\left(\frac{k_{\mathrm{B}} T}{2 J}\right)^{2 n+1} \frac{2 a_{n}}{(2 n) !} \frac{d^{2 n}}{d \omega^{2 n}}\left[k(\omega)-\frac{1}{4}\right](\omega-\mu)\right|_{\omega=\mu}+\mathcal{O}\left(e^{-\beta}\right) \\
& =\frac{a_{0} k_{\mathrm{B}} T}{J}\left(k_{\mathrm{F}}-\frac{1}{4}\right) \cdot 0+\left(\frac{k_{\mathrm{B}} T}{2 J}\right)^{3} \cdot \frac{2 a_{1}}{2}\left[k_{\mathrm{F}}^{\prime \prime} \cdot 0+k_{\mathrm{F}}^{\prime}+\left(k_{\mathrm{F}}-\frac{1}{4}\right) \cdot 0\right]+\mathcal{O}\left(T^{5}\right) \\
& =\frac{a_{1} k_{\mathrm{B}}^{3}}{8 J^{3}} k_{\mathrm{F}}^{\prime} T^{3}+\mathcal{O}\left(T^{5}\right) \\
& =\frac{a_{1} k_{\mathrm{B}}^{3}}{16 \pi J^{3}} T^{3}+\mathcal{O}\left(\varepsilon(\mu-\varepsilon) T^{3}\right)+\mathcal{O}\left(T^{5}\right)
\end{aligned}
$$


for small temperature $T$. Here is exactly one leading term in contrast to $\left\langle\tilde{\chi}^{(3)}, j^{N}\right\rangle_{\mathrm{F}}$. This leads to Eq. (3.130).

For the calculation of the expectation values $\left\langle\hat{J}^{N}\right\rangle_{t}$ and $\left\langle\hat{J}^{Q}\right\rangle_{t}$ (see Eq. (3.131) and Eq. (3.131), respectively), we additionally need to consider the norm of the approximate eigenfunction:

$$
\begin{aligned}
\left\|\tilde{\chi}^{(3)}\right\|_{\mathrm{F}}^{2}= & \frac{k_{\mathrm{B}} T}{2 J} a_{0} k_{\mathrm{F}}^{\prime}\left[k_{\mathrm{F}}-\frac{1}{4}\right]^{2}+\left(\frac{k_{\mathrm{B}} T}{2 J}\right)^{3} \frac{1}{2} a_{1}\left\{k_{\mathrm{F}}^{\prime \prime \prime}\left[k_{\mathrm{F}}-\frac{1}{4}\right]^{2}\right. \\
& \left.+4 k_{\mathrm{F}}^{\prime \prime} k_{\mathrm{F}}^{\prime}\left[k_{\mathrm{F}}-\frac{1}{4}\right]+2\left(k_{\mathrm{F}}^{\prime}\right)^{3}\right\}+\mathcal{O}\left(e^{-\beta}\right) \\
= & \frac{k_{\mathrm{B}} T}{2 J} a_{0} k_{\mathrm{F}}^{\prime}\left[k_{\mathrm{F}}-\frac{1}{4}\right]^{2}+\frac{a_{1} k_{\mathrm{B}}^{3}}{8 J^{3}}\left(k_{\mathrm{F}}^{\prime}\right)^{3} T^{3}+\mathcal{O}\left(T^{5}\right) .
\end{aligned}
$$

This leads to

$$
\begin{aligned}
\left\|\tilde{\chi}^{(3)}\right\|_{\mathrm{F}}^{2} & = \begin{cases}\frac{a_{0} k_{\mathrm{B}}}{2 J} k_{\mathrm{F}}^{\prime}\left[k_{\mathrm{F}}-\frac{1}{4}\right]^{2} T+\mathcal{O}\left(T^{3}\right) & \text { for }\left(k_{\mathrm{B}} T\right)^{2} \ll|\mu-\varepsilon|^{2} \\
\frac{a_{1} k_{\mathrm{B}}^{3}}{8 J^{3}}\left(k_{\mathrm{F}}^{\prime}\right)^{3} T^{3}+\mathcal{O}\left(T^{5}\right) & \text { for }|\mu-\varepsilon|^{2} \ll\left(k_{\mathrm{B}} T\right)^{2}\end{cases} \\
& = \begin{cases}\frac{a_{0} k_{\mathrm{B}}}{16 \pi^{3} J} \arcsin ^{2}(\varepsilon-\mu) T+\mathcal{O}\left(T^{3}\right) & \text { for }\left(k_{\mathrm{B}} T\right)^{2} \ll|\mu-\varepsilon|^{2} \\
\frac{a_{1} k_{\mathrm{B}}^{3}}{64 \pi^{3} J^{3}} T^{3}+\mathcal{O}\left(T^{5}\right) & \text { for }|\mu-\varepsilon|^{2} \ll\left(k_{\mathrm{B}} T\right)^{2} .\end{cases}
\end{aligned}
$$

We also need to consider the scalar product with the initial perturbation $\phi_{0}(k)$ :

$$
\begin{aligned}
\left\langle\tilde{\chi}^{(3)}, \phi_{0}\right\rangle_{\mathrm{F}} & =\left(\frac{k_{\mathrm{B}} T}{2 J}\right) a_{0} k_{\mathrm{F}}^{\prime}\left[k_{\mathrm{F}}-\frac{1}{4}\right]\left[\phi_{0}\left(k_{\mathrm{F}}\right)-\phi_{0}\left(-k_{\mathrm{F}}\right)\right] \\
+ & \left(\frac{k_{\mathrm{B}} T}{2 J}\right)^{3} \frac{1}{2} a_{1}\left\{k_{\mathrm{F}}^{\prime \prime \prime}\left[k_{\mathrm{F}}-\frac{1}{4}\right]\left[\phi_{0}\left(k_{\mathrm{F}}\right)-\phi_{0}\left(-k_{\mathrm{F}}\right)\right]\right. \\
& +2 k_{\mathrm{F}}^{\prime \prime} k_{\mathrm{F}}^{\prime}\left[\phi_{0}\left(k_{\mathrm{F}}\right)-\phi_{0}\left(-k_{\mathrm{F}}\right)\right]+2 k_{\mathrm{F}}^{\prime \prime}\left[k_{\mathrm{F}}-\frac{1}{4}\right]\left[\phi_{0}^{\prime}\left(k_{\mathrm{F}}\right)+\phi_{0}^{\prime}\left(-k_{\mathrm{F}}\right)\right] k_{\mathrm{F}}^{\prime} \\
& +k_{\mathrm{F}}^{\prime} k_{\mathrm{F}}^{\prime \prime}\left[\phi_{0}\left(k_{\mathrm{F}}\right)-\phi_{0}\left(-k_{\mathrm{F}}\right)\right]+2 k_{\mathrm{F}}^{\prime} k_{\mathrm{F}}^{\prime}\left[\phi_{0}^{\prime}\left(k_{\mathrm{F}}\right)+\phi_{0}^{\prime}\left(-k_{\mathrm{F}}\right)\right] \\
& +k_{\mathrm{F}}^{\prime}\left[k_{\mathrm{F}}-\frac{1}{4}\right]\left[\phi_{0}^{\prime \prime}\left(k_{\mathrm{F}}\right)-\phi_{0}^{\prime \prime}\left(-k_{\mathrm{F}}\right)\right]\left(k_{\mathrm{F}}^{\prime}\right)^{2} \\
& \left.+k_{\mathrm{F}}^{\prime}\left[k_{\mathrm{F}}-\frac{1}{4}\right]\left[\phi_{0}^{\prime}\left(k_{\mathrm{F}}\right)+\phi_{0}^{\prime}\left(-k_{\mathrm{F}}\right)\right] k_{\mathrm{F}}^{\prime \prime}\right\}+\mathcal{O}\left(T^{5}\right) .
\end{aligned}
$$

As in previous scalar products, one can also distinguish between two leading 
terms provided that $\phi_{0}(k)$ has an anti-symmetric part:

$$
\begin{aligned}
& \left\langle\tilde{\chi}^{(3)}, \phi_{0}\right\rangle_{\mathrm{F}}= \\
& = \begin{cases}\frac{a_{0} k_{\mathrm{B}}}{2 J} k_{\mathrm{F}}^{\prime}\left[k_{\mathrm{F}}-\frac{1}{4}\right]\left[\phi_{0}\left(k_{\mathrm{F}}\right)-\phi_{0}\left(-k_{\mathrm{F}}\right)\right] T+\mathcal{O}\left(T^{3}\right) & \text { for }\left(k_{\mathrm{B}} T\right)^{2} \ll|\mu-\varepsilon| \\
\frac{a_{1} k_{\mathrm{B}}^{3}}{8 J^{3}}\left(k_{\mathrm{F}}^{\prime}\right)^{2}\left[\phi_{0}^{\prime}\left(k_{\mathrm{F}}\right)+\phi_{0}^{\prime}\left(-k_{\mathrm{F}}\right)\right] T^{3}+\mathcal{O}\left(T^{5}\right) & \text { for }|\mu-\varepsilon| \ll\left(k_{\mathrm{B}} T\right)^{2}\end{cases} \\
& =\left\{\begin{aligned}
\frac{a_{0} k_{\mathrm{B}}}{8 \pi^{2} J} \arcsin (\mu-\varepsilon)\left[\phi_{0}\left(k_{\mathrm{F}}\right)-\phi_{0}\left(-k_{\mathrm{F}}\right)\right] T+\mathcal{O}\left(\varepsilon(\mu-\varepsilon)^{2} T\right)+\mathcal{O}\left(T^{3}\right) \\
\text { for }\left(k_{\mathrm{B}} T\right)^{2} \ll|\mu-\varepsilon| \\
\frac{a_{1} k_{\mathrm{B}}^{3}}{32 \pi^{2} J^{3}}\left[\phi_{0}^{\prime}\left(k_{\mathrm{F}}\right)+\phi_{0}^{\prime}\left(-k_{\mathrm{F}}\right)\right] T^{3}+\mathcal{O}\left(\varepsilon(\mu-\varepsilon) T^{3}\right)+\mathcal{O}\left(T^{5}\right) \\
\text { for }|\mu-\varepsilon| \ll\left(k_{\mathrm{B}} T\right)^{2} .
\end{aligned}\right.
\end{aligned}
$$

Note that here we assumed $\phi_{0}\left(k_{\mathrm{F}}\right)-\phi_{0}\left(-k_{\mathrm{F}}\right) \neq 0$ and $\phi_{0}^{\prime}\left(k_{\mathrm{F}}\right)+\phi_{0}^{\prime}\left(-k_{\mathrm{F}}\right) \neq 0$. If this is not the case, other terms are the leading ones. Then, another calculation is necessary.

This is the case for our last calculation, where we consider the function $\phi_{0}^{\text {ex.2 }}(k):=$ $\operatorname{sgn}(k) \cdot(\omega(k)-\mu)$, which we find in one of our examples for a physically meaningful initial perturbation, see Eq. (3.84). Using the Sommerfeld expansion in Eq. (B.82), we obtain

$$
\begin{aligned}
\left\langle\chi^{(3)}, \phi_{0}^{\text {ex.2}}\right\rangle_{\mathrm{F}}= & \sum_{n=0}^{\infty}\left(\frac{k_{\mathrm{B}} T}{2 J}\right)^{2 n+1} \frac{a_{n}}{(2 n) !} \frac{d^{2 n}}{d \tilde{\omega}^{2 n}} 2 k^{\prime}(\tilde{\omega})\left[k(\tilde{\omega})-\frac{1}{4} \operatorname{sgn}(k(\tilde{\omega}))\right] \\
& \times\left.\operatorname{sgn}(k(\tilde{\omega}))[\omega(k(\tilde{\omega}))-\mu]\right|_{\tilde{\omega}=\mu}+\mathcal{O}\left(e^{-\beta}\right) .
\end{aligned}
$$

With all the derivatives of $\operatorname{sgn}(k)$ vanishing at $k=k_{\mathrm{F}}$, we obtain

$$
\begin{aligned}
\left\langle\chi^{(3)}, \phi_{0}^{\mathrm{ex} .2}\right\rangle_{\mathrm{F}}=\left.\sum_{n=0}^{\infty}\left(\frac{k_{\mathrm{B}} T}{2 J}\right)^{2 n+1} \frac{2 a_{n}}{(2 n) !} \frac{d^{2 n}}{d \omega^{2 n}} k^{\prime}(\omega)\left[k(\omega)-\frac{1}{4}\right](\omega-\mu)\right|_{\omega=\mu}+\mathcal{O}\left(e^{-\beta}\right) \\
=\frac{a_{0} k_{\mathrm{B}} T}{J} k_{\mathrm{F}}^{\prime}\left(k_{\mathrm{F}}-\frac{1}{4}\right) \cdot 0+\left(\frac{k_{\mathrm{B}} T}{2 J}\right)^{3} \cdot \frac{2 a_{1}}{2}\left[k_{\mathrm{F}}^{\prime \prime \prime}\left(k_{\mathrm{F}}-\frac{1}{4}\right) \cdot 0+k_{\mathrm{F}}^{\prime} k_{\mathrm{F}}^{\prime \prime} \cdot 0\right. \\
\left.\quad+k_{\mathrm{F}}^{\prime}\left(k_{\mathrm{F}}-\frac{1}{4}\right) \cdot 0+k_{\mathrm{F}}^{\prime \prime} k_{\mathrm{F}}^{\prime} \cdot 0+k_{\mathrm{F}}^{\prime \prime}\left(k_{\mathrm{F}}-\frac{1}{4}\right)+k_{\mathrm{F}}^{\prime} k_{\mathrm{F}}^{\prime}\right]+\mathcal{O}\left(T^{5}\right) \\
=\frac{a_{1} k_{\mathrm{B}}^{3}}{8 J^{3}}\left[\left(k_{\mathrm{F}}^{\prime}\right)^{2}+k_{\mathrm{F}}^{\prime \prime}\left(k_{\mathrm{F}}-\frac{1}{4}\right)\right] T^{3}+\mathcal{O}\left(T^{5}\right) \\
=\frac{a_{1} k_{\mathrm{B}}^{3}}{32 \pi^{2} J^{3}}[1+(\mu-\varepsilon) \arcsin (\mu-\varepsilon)] T^{3}+\mathcal{O}\left(\varepsilon(\mu-\varepsilon) T^{3}\right)+\mathcal{O}\left(T^{5}\right) .
\end{aligned}
$$

This is used in Eqs. (C.74) and (C.79).

\section{C.5 Particle and energy current}

We calculate the total particle current $\hat{J}^{N}=\sum_{l} \hat{l}_{l}^{N}$ and the total energy current $\hat{J}^{E}=\sum_{l} \hat{\jmath}_{l}^{E}$. In order to do this, we need to use the well-known continuity 
equation. It links the local particle density $\rho_{l}^{(N)}:=\hat{n}_{l}=\sum_{\sigma} \hat{n}_{\sigma, l}$ to the local particle current $\hat{\jmath}_{l}^{N}$ and the local energy density

$$
\rho_{l}^{(E)}:=\hat{h}_{l}=\hat{h}_{l}^{\mathrm{kin}}+\hat{h}_{l}^{\mathrm{int}}=\left(-J \hat{q}_{\sigma l}^{(1)}-J^{\prime} \hat{q}_{\sigma l}^{(2)}\right)+H . c .+U \hat{n}_{\uparrow, l} \hat{n}_{\downarrow, l}
$$

to the local energy current $\hat{\jmath}_{l}^{E}$. The discretized continuity equations (Essler et al., 2005) read

$$
\frac{d}{d t} \rho_{l}^{(X)}=\hat{\jmath}_{l-1}^{X}-\hat{\jmath}_{l}^{X},
$$

with $X \in\{N, E\}$. Utilizing a known simple trick, we can use this to directly calculate the total currents:

$$
\hat{J}^{X}=\sum_{l} \hat{\jmath}_{l}^{X}[(l+1)-l]=\sum_{l} l\left[\hat{\jmath}_{l-1}^{X}-\hat{\jmath}_{l}^{X}\right]=\sum_{l} \frac{d}{d t} \rho_{l}^{(X)}=\frac{i}{\hbar} \sum_{l} l\left[\rho_{l}^{(X)}, H\right] .
$$

In order to perform the calculation and keep track of what we are doing, we define a short notation:

$$
\begin{aligned}
& \hat{q}_{\sigma l}^{(n)}:=\hat{c}_{\sigma, l+n}^{\dagger} \hat{c}_{\sigma, l}+\hat{c}_{\sigma, l}^{\dagger} \hat{c}_{\sigma, l+n} \\
& \zeta_{\sigma l}^{(n)}:=\hat{c}_{\sigma, l+n}^{\dagger} \hat{c}_{\sigma, l}-\hat{c}_{\sigma, l}^{\dagger} \hat{c}_{\sigma, l+n} .
\end{aligned}
$$

Using $\hat{H}=\sum_{l} \hat{h}_{l}$, the total particle current becomes

$$
\hat{J}^{N}=\frac{i}{\hbar} \sum_{\sigma, l} l\left[\hat{n}_{\sigma, l},-J \hat{q}_{\sigma m}^{(1)}-J^{\prime} \hat{q}_{\sigma m}^{(2)}\right] .
$$

For the evaluation of this equation, we make use of

$$
\left[\hat{c}_{\alpha}^{\dagger} \hat{c}_{\beta}, \hat{c}_{\gamma}^{\dagger} \hat{c}_{\eta}\right]=\hat{c}_{\alpha}^{\dagger} \hat{c}_{\eta} \delta_{\beta \gamma}-\hat{c}_{\gamma}^{\dagger} \hat{c}_{\beta} \delta_{\alpha \eta}
$$

and

$$
\begin{aligned}
{\left[\hat{n}_{\sigma j}, \hat{q}_{\sigma n}^{(a)}\right] } & =\left[\hat{c}_{\sigma, j}^{\dagger} \hat{c}_{\sigma, j}, \hat{c}_{\sigma, n+a}^{\dagger} \hat{c}_{\sigma, n}+\hat{c}_{\sigma, n}^{\dagger} \hat{c}_{\sigma, n+a}\right] \\
& =\hat{c}_{\sigma, j}^{\dagger} \hat{c}_{\sigma, n} \delta_{j, n+a}-\hat{c}_{\sigma, n+a}^{\dagger} \hat{c}_{\sigma, j} \delta_{j n}+\hat{c}_{\sigma, j}^{\dagger} \hat{c}_{\sigma, n+a} \delta_{j n}-\hat{c}_{\sigma, n}^{\dagger} \hat{c}_{\sigma, j} \delta_{j, n+a} \\
& =\zeta_{\sigma n}^{(a)}\left(\delta_{j, n+a}-\delta_{j n}\right) .
\end{aligned}
$$

Hence, Eq. (C.25) becomes

$$
\begin{aligned}
\hat{J}^{N} & =-\frac{i}{\hbar} \sum_{\sigma, l} l\left[J \zeta_{\sigma m}^{(1)}\left(\delta_{l, m+1}-\delta_{l m}\right)+J^{\prime} \zeta_{\sigma m}^{(2)}\left(\delta_{l, m+2}-\delta_{l m}\right)\right] \\
& =-\frac{i}{\hbar} \sum_{\sigma, l} l\left[J\left(\zeta_{\sigma l-1}^{(1)}-\zeta_{\sigma l}^{(1)}\right)+J^{\prime}\left(\zeta_{\sigma l-2}^{(2)}-\zeta_{\sigma l}^{(2)}\right)\right] .
\end{aligned}
$$

Shifting $l$ in the first and third term by 1 and 2, respectively, one obtains

$$
\begin{aligned}
\hat{J}^{N} & =-\frac{i}{\hbar} \sum_{\sigma, l}\left\{J \zeta_{\sigma l}^{(1)}[(l+1)-l]+J^{\prime} \zeta_{\sigma l}^{(2)}[(l+2)-l]\right\} \\
& =-\frac{i}{\hbar} \sum_{\sigma, l}\left(J \zeta_{\sigma l}^{(1)}+2 J^{\prime} \zeta_{\sigma l}^{(2)}\right) .
\end{aligned}
$$


We can express this with the group velocity $\omega^{\prime}(k)=2 \pi[\sin (2 \pi k)+2 \varepsilon \sin (4 \pi k)]$ by transforming the current into momentum space by employing

$$
\begin{aligned}
\sum_{m} \zeta_{\sigma m}^{(a)} & =\sum_{m} c_{m+a}^{\dagger} c_{m}-H . c .=\int d k e^{i 2 \pi k a} \hat{n}(k)-H . c . \\
& =2 i \int d k \sin (2 \pi k a) \hat{n}(k) .
\end{aligned}
$$

This leads to

$$
\hat{J}^{N}=\frac{2}{\hbar} \sum_{\sigma} \int d k\left[J \sin (2 \pi k)+2 J^{\prime} \sin (4 \pi k)\right] \hat{n}(k) .
$$

Now, we can use $\omega^{\prime}(k)$ and finally obtain:

$$
\hat{J}^{N}=\frac{J}{\pi \hbar} \sum_{\sigma} \int d k \omega^{\prime}(k) \hat{n}(k)
$$

The second current, which we will calculate, is the energy current. From Eq. (C.23), we deduce:

$$
\begin{aligned}
\hat{J}^{E} & =\frac{i}{\hbar} \sum_{l m} l\left[\hat{h}_{l}, \hat{h}_{m}\right] \\
= & \frac{i}{\hbar} \sum_{\sigma l m} l\left[-J \hat{q}_{\sigma l}^{(1)}-J^{\prime} \hat{q}_{\sigma l}^{(2)}+U \hat{n}_{\uparrow l} \hat{n}_{\downarrow l},-J \hat{q}_{\sigma m}^{(1)}-J^{\prime} \hat{q}_{\sigma m}^{(2)}+U \hat{n}_{\uparrow m} \hat{n}_{\downarrow m}\right] \\
= & \frac{i}{\hbar} \sum_{\sigma l m} l\left\{J^{2}\left[\hat{q}_{\sigma l}^{(1)}, \hat{q}_{\sigma m}^{(1)}\right]-J J^{\prime}\left(\left[\hat{q}_{\sigma l}^{(1)}, \hat{q}_{\sigma m}^{(2)}\right]+\left[\hat{q}_{\sigma l}^{(2)}, \hat{q}_{\sigma m}^{(1)}\right]\right)+\left(J^{\prime}\right)^{2}\left[\hat{q}_{\sigma l}^{(2)}, \hat{q}_{\sigma m}^{(2)}\right]\right. \\
& -J U\left(\left[\hat{q}_{\sigma l}^{(1)}, \hat{n}_{\uparrow m} \hat{n}_{\downarrow m}\right]+\left[\hat{n}_{\uparrow l} \hat{n}_{\downarrow l}, \hat{q}_{\sigma m}^{(1)}\right]\right)-J U\left(\left[\hat{q}_{\sigma l}^{(2)}, \hat{n}_{\uparrow m} \hat{n}_{\downarrow m}\right]+\left[\hat{n}_{\uparrow l} \hat{n}_{\downarrow l}, \hat{q}_{\sigma m}^{(2)}\right]\right) \\
& \left.+U^{2}\left[\hat{n}_{\uparrow l} \hat{n}_{\downarrow l}, \hat{n}_{\uparrow m} \hat{n}_{\downarrow m}\right]\right\} .
\end{aligned}
$$

The last term vanishes and the remaining interaction terms can be simplified by defining $\bar{\sigma}$, which denotes the opposite spin of $\sigma$ :

$$
\begin{aligned}
\hat{J}^{E} & =\frac{i J^{2}}{\hbar} \sum_{\sigma l m} l\left\{\left[\hat{q}_{\sigma l}^{(1)}, \hat{q}_{\sigma m}^{(1)}\right]+\varepsilon\left(\left[\hat{q}_{\sigma l}^{(1)}, \hat{q}_{\sigma m}^{(2)}\right]+\left[\hat{q}_{\sigma l}^{(2)}, \hat{q}_{\sigma m}^{(1)}\right]\right)+\varepsilon^{2}\left[\hat{q}_{\sigma l}^{(2)}, \hat{q}_{\sigma m}^{(2)}\right]\right. \\
& \left.-u\left(\left[\hat{q}_{\sigma l}^{(1)}, \hat{n}_{\sigma m}\right] \hat{n}_{\bar{\sigma} m}+\left[\hat{n}_{\sigma l}, \hat{q}_{\sigma m}^{(1)}\right] \hat{n}_{\bar{\sigma} l}\right)-\varepsilon u\left(\left[\hat{q}_{\sigma l}^{(2)}, \hat{n}_{\sigma m}\right] \hat{n}_{\bar{\sigma} m}+\left[\hat{n}_{\sigma l}, \hat{q}_{\sigma m}^{(2)}\right] \hat{n}_{\bar{\sigma} l}\right)\right\},
\end{aligned}
$$

where $\varepsilon=J^{\prime} / J$ and $u=U / J$. For this, we need to calculate the missing commutators:

$$
\begin{aligned}
& {\left[\hat{q}_{\sigma j}^{(a)}, \hat{q}_{\sigma n}^{(b)}\right]=\left[\hat{c}_{\sigma, j+a}^{\dagger} \hat{c}_{\sigma, j}+\hat{c}_{\sigma, j}^{\dagger} \hat{c}_{\sigma, j+a}, \hat{c}_{\sigma, n+b}^{\dagger} \hat{c}_{\sigma, n}+H . c .\right]} \\
& =\left(\hat{c}_{\sigma, j+a}^{\dagger} \hat{c}_{\sigma, n} \delta_{j, n+b}-\hat{c}_{\sigma, n+b}^{\dagger} \hat{c}_{\sigma, j} \delta_{j+a, n}+\hat{c}_{\sigma, j}^{\dagger} \hat{c}_{\sigma, n} \delta_{j+a, n+b}-\hat{c}_{\sigma, n+b}^{\dagger} \hat{c}_{\sigma, j+a} \delta_{j n}\right)-H . c . \\
& =\zeta_{\sigma n}^{(a+b)} \delta_{j, n+b}-\zeta_{\sigma j}^{(a+b)} \delta_{j+a, n}+\zeta_{\sigma n}^{(b-a)} \delta_{j+a, n+b}-\zeta_{\sigma, n+a}^{(b-a)} \delta_{j n} .
\end{aligned}
$$


We realize that $\zeta_{\sigma n}^{(0)} \equiv 0$ leads to

$$
\begin{aligned}
& {\left[\hat{q}_{\sigma l}^{(1)}, \hat{q}_{\sigma m}^{(1)}\right]=\zeta_{\sigma m}^{(2)} \delta_{l, m+2}-\zeta_{\sigma l}^{(2)} \delta_{l, m-2}} \\
& {\left[\hat{q}_{\sigma l}^{(2)}, \hat{q}_{\sigma m}^{(2)}\right]=\zeta_{\sigma m}^{(4)} \delta_{l, m+4}-\zeta_{\sigma l}^{(4)} \delta_{l, m-4} .}
\end{aligned}
$$

Furthermore, we get

$$
\left[\hat{q}_{\sigma j}^{(1)}, \hat{q}_{\sigma n}^{(2)}\right]=\zeta_{\sigma n}^{(3)} \delta_{j, n+2}-\zeta_{\sigma j}^{(3)} \delta_{j, n-1}+\zeta_{\sigma n}^{(1)} \delta_{j, n+1}-\zeta_{\sigma, n+1}^{(1)} \delta_{j n} .
$$

Plugging these commutators into Eq. (C.34), it gets the form

$$
\begin{aligned}
\hat{J}^{E}= & \frac{i J^{2}}{\hbar} \sum_{\sigma l m} l\left\{\left(\zeta_{\sigma m}^{(2)} \delta_{l, m+2}-\zeta_{\sigma l}^{(2)} \delta_{l, m-2}\right)+\varepsilon^{2}\left(\zeta_{\sigma m}^{(4)} \delta_{l, m+4}-\zeta_{\sigma l}^{(4)} \delta_{l, m-4}\right)\right. \\
& +\varepsilon\left(\zeta_{\sigma m}^{(3)} \delta_{l, m+2}-\zeta_{\sigma l}^{(3)} \delta_{l, m-1}+\zeta_{\sigma m}^{(1)} \delta_{l, m+1}-\zeta_{\sigma, m+1}^{(1)} \delta_{l m}\right. \\
& \left.-\zeta_{\sigma l}^{(3)} \delta_{m, l+2}+\zeta_{\sigma m}^{(3)} \delta_{m, l-1}-\zeta_{\sigma l}^{(1)} \delta_{m, l+1}+\zeta_{\sigma, l+1}^{(1)} \delta_{m l}\right) \\
& -u\left(-\zeta_{\sigma l}^{(1)}\left(\delta_{m, l+1}-\delta_{m l}\right) \hat{n}_{\bar{\sigma} m}+\zeta_{\sigma m}^{(1)}\left(\delta_{l, m+1}-\delta_{l m}\right) \hat{n}_{\bar{\sigma} l}\right) \\
& \left.-\varepsilon u\left(-\zeta_{\sigma l}^{(2)}\left(\delta_{m, l+2}-\delta_{m l}\right) \hat{n}_{\bar{\sigma} m}+\zeta_{\sigma m}^{(2)}\left(\delta_{l, m+2}-\delta_{l m}\right) \hat{n}_{\bar{\sigma} l}\right)\right\}
\end{aligned}
$$

Now, we perform the sum over $m$ :

$$
\begin{aligned}
\hat{J}^{E}= & \frac{i J^{2}}{\hbar} \sum_{\sigma l} l\left\{\left(\zeta_{\sigma l-2}^{(2)}-\zeta_{\sigma l}^{(2)}\right)+\varepsilon^{2}\left(\zeta_{\sigma l-4}^{(4)}-\zeta_{\sigma l}^{(4)}\right)\right. \\
& -\varepsilon\left(\zeta_{\sigma, l-2}^{(3)}-\zeta_{\sigma l}^{(3)}+\zeta_{\sigma, l-1}^{(1)}-\zeta_{\sigma, l+1}^{(1)}-\zeta_{\sigma l}^{(3)}+\zeta_{\sigma, l-1}^{(3)}-\zeta_{\sigma l}^{(1)}+\zeta_{\sigma, l+1}^{(1)}\right) \\
& -u\left(-\zeta_{\sigma l}^{(1)}\left(\hat{n}_{\bar{\sigma}, l+1}-\hat{\eta}_{\bar{\sigma} l}\right)+\left(\zeta_{\sigma, l-1}^{(1)}-\zeta_{\sigma l}^{(2)}\right) \hat{n}_{\bar{\sigma} l}\right) \\
& \left.-\varepsilon u\left(-\zeta_{\sigma l}^{(2)}\left(\hat{n}_{\bar{\sigma}, l+2}-\hat{\eta}_{\bar{\sigma} l}\right)+\left(\zeta_{\sigma, l-2}^{(2)}-\zeta_{\sigma l}^{(2)}\right) \hat{n}_{\bar{\sigma} l}\right)\right\} .
\end{aligned}
$$

As a next step, we collect like terms by shifting $l$ in some terms by 1 or 2 :

$$
\begin{aligned}
\hat{J}^{E}= & \frac{i J^{2}}{\hbar} \sum_{\sigma l}\left\{\zeta_{\sigma l}^{(2)}[(l+2)-l]+\varepsilon^{2} \zeta_{\sigma l}^{(4)}[(l+4)-l]\right. \\
& -\varepsilon\left\{\zeta_{\sigma l}^{(3)}[(l+2)-l-l+(l+1)]+\zeta_{\sigma l}^{(1)}[(l+1)-l]\right\} \\
& \left.+u \zeta_{\sigma l}^{(1)} \hat{n}_{\bar{\sigma}, l+1}[l-(l+1)]+\varepsilon u \zeta_{\sigma l}^{(2)} \hat{n}_{\bar{\sigma}, l+2}[l-(l+2)]\right\} .
\end{aligned}
$$

This can be simplified to

$$
\hat{J}^{E}=\frac{i J^{2}}{\hbar} \sum_{\sigma l}\left\{2 \zeta_{\sigma l}^{(2)}+4 \varepsilon^{2} \zeta_{\sigma l}^{(4)}+\varepsilon\left(3 \zeta_{\sigma l}^{(3)}+\zeta_{\sigma l}^{(1)}\right)-u \zeta_{\sigma l}^{(1)} \hat{n}_{\bar{\sigma}, l+1}-2 \varepsilon u \zeta_{\sigma l}^{(2)} \hat{n}_{\bar{\sigma}, l+2}\right\} .
$$

The terms, which remain when $u=0$, can be further rewritten with the dispersion relation $\omega(k)$ using the transformation to momentum space in Eq. (C.30):

$$
\begin{aligned}
& \sum_{\sigma l}\left\{2 \zeta_{\sigma l}^{(2)}+4 \varepsilon^{2} \zeta_{\sigma l}^{(4)}+\varepsilon\left(3 \zeta_{\sigma l}^{(3)}+\zeta_{\sigma l}^{(1)}\right)\right\} \\
& =\sum_{\sigma} \int d k\left\{4 i \sin (4 \pi k)+8 i \varepsilon^{2} \sin (8 \pi k)+\varepsilon[6 i \sin (6 \pi k)+2 i \sin (2 \pi k)]\right\} \hat{n}_{\sigma}(k) .
\end{aligned}
$$


We can show that the integrand is proportional to $\omega(k) \omega^{\prime}(k)$ :

$$
\begin{aligned}
& \omega(k) \omega^{\prime}(k)= \\
&=- 2 \pi[\cos (2 \pi k)+\varepsilon \cos (4 \pi k)][\sin (2 \pi k)+2 \varepsilon \sin (4 \pi k)] \\
&=- 2 \pi\left\{\frac{1}{2} \sin (4 \pi k)+4 \varepsilon \sin (2 \pi k) \cos ^{2}(2 \pi k)\right. \\
&\left.\quad+\varepsilon \sin (2 \pi k)\left[2 \cos ^{2}(2 \pi k)-1\right]+\varepsilon^{2} \sin (8 \pi k)\right\} \\
&=-\pi\left\{\sin (4 \pi k)+12 \varepsilon \sin (2 \pi k) \cos ^{2}(2 \pi k)-2 \varepsilon \sin (2 \pi k)+2 \varepsilon^{2} \sin (8 \pi k)\right\} .
\end{aligned}
$$

Using $\sin (6 \pi k)=4 \sin (2 \pi k) \cos ^{2}(2 \pi k)-\sin (2 \pi k)$, we obtain

$$
\omega(k) \omega^{\prime}(k)=-\pi\left[\sin (4 \pi k)+3 \varepsilon \sin (6 \pi k)+\varepsilon \sin (2 \pi k)+2 \varepsilon^{2} \sin (8 \pi k)\right] .
$$

This leads to the final form of the total energy current

$$
\hat{J}^{E}=\frac{J}{\hbar} \sum_{\sigma}\left\{\frac{4 J}{\pi} \int d k \omega(k) \omega^{\prime}(k) \hat{n}_{\sigma}(k)-U \sum_{l}\left[\zeta_{\sigma l}^{(1)} \hat{n}_{\bar{\sigma}, l+1}+2 \varepsilon \zeta_{\sigma l}^{(2)} \hat{n}_{\bar{\sigma}, l+2}\right]\right\} .
$$

\section{C.6 Calculation of the total currents' expecta- tion values}

In this appendix, we calculate the expectation values $\left\langle\hat{J}^{N}\right\rangle_{t}$ and $\left\langle\hat{J}^{Q}\right\rangle_{t}$, see Eq. (3.131) and Eq. (3.131), respectively. In App. C.6.1, we consider a general initial perturbation $\phi_{0}$ and assume that $\phi_{0}\left(k_{\mathrm{F}}\right)-\phi_{0}\left(-k_{\mathrm{F}}\right) \neq 0$ and $\phi_{0}^{\prime}\left(k_{\mathrm{F}}\right)+$ $\phi_{0}^{\prime}\left(-k_{\mathrm{F}}\right) \neq 0$. The last part, App. C.6.2, uses the two examples for a physically meaningful initial perturbation found in Sec. 3.2.5.

\section{C.6.1 General initial perturbation}

First, we consider the expectation value of the total particle current and make use of Eq. (3.128):

$$
\left\langle\hat{J}^{N}\right\rangle_{t}=e^{-\lambda_{3} t} \frac{\left\langle\phi_{0}, \chi^{(3)}\right\rangle_{\mathrm{F}}\left\langle\chi^{(3)}, j^{N}\right\rangle_{\mathrm{F}}}{\left\|\chi^{(3)}\right\|_{\mathrm{F}}^{2}}
$$

As a next step, we use the Sommerfeld expansions of the occurring scalar products calculated in Eqs. (C.14), (C.16), and (C.18):

$$
\begin{aligned}
\left\langle\hat{J}^{N}\right\rangle_{t}=e^{-\lambda_{3} t} & \left\{\frac{a_{0} k_{\mathrm{B}}}{2 J} k_{\mathrm{F}}^{\prime}\left[k_{\mathrm{F}}-\frac{1}{4}\right]\left[\phi_{0}\left(k_{\mathrm{F}}\right)-\phi_{0}\left(-k_{\mathrm{F}}\right)\right] T\right. \\
& \left.+\frac{a_{1} k_{\mathrm{B}}^{3}}{8 J^{3}}\left(k_{\mathrm{F}}^{\prime}\right)^{2}\left[\phi_{0}^{\prime}\left(k_{\mathrm{F}}\right)+\phi_{0}^{\prime}\left(-k_{\mathrm{F}}\right)\right] T^{3}+\mathcal{O}\left(T^{5}\right)\right\} \\
& \times \frac{\left[\frac{a_{0} k_{\mathrm{B}}}{2 J}\left(k_{\mathrm{F}}-\frac{1}{4}\right) T+\frac{a_{1} k_{\mathrm{B}}^{3}}{16 J^{3}} k_{\mathrm{F}}^{\prime \prime} T^{3}+\mathcal{O}\left(T^{5}\right)\right]}{\left[\frac{a_{0} k_{\mathrm{B}}}{2 J} k_{\mathrm{F}}^{\prime}\left[k_{\mathrm{F}}-\frac{1}{4}\right]^{2} T+\frac{a_{1} k_{\mathrm{B}}^{3}}{8 J^{3}}\left(k_{\mathrm{F}}^{\prime}\right)^{3} T^{3}+\mathcal{O}\left(T^{5}\right)\right]} .
\end{aligned}
$$


Furthermore, we obtain the leading terms of the different temperature regimes

$$
\left\langle\hat{J}^{N}\right\rangle_{t}=e^{-\lambda_{3} t}\left\{\begin{array}{l}
\frac{a_{0} k_{\mathrm{B}}}{2 J}\left[\phi_{0}\left(k_{\mathrm{F}}\right)-\phi_{0}\left(-k_{\mathrm{F}}\right)\right] T \\
\text { for }\left(k_{\mathrm{B}} T\right)^{2} \ll|\mu-\varepsilon| \wedge \varepsilon\left(k_{\mathrm{B}} T\right)^{2} \ll|\mu-\varepsilon| \wedge\left(k_{\mathrm{B}} T\right)^{2} \ll|\mu-\varepsilon|^{2} \\
\frac{2 J a_{0}^{2}}{a_{1} k_{\mathrm{B}}}\left(\frac{k_{\mathrm{F}}-\frac{1}{4}}{k_{\mathrm{F}}^{\prime}}\right)^{2}\left[\phi_{0}\left(k_{\mathrm{F}}\right)-\phi_{0}\left(-k_{\mathrm{F}}\right)\right] T^{-1} \\
\text { for }\left(k_{\mathrm{B}} T\right)^{2} \ll|\mu-\varepsilon| \wedge \varepsilon\left(k_{\mathrm{B}} T\right)^{2} \ll|\mu-\varepsilon| \wedge|\mu-\varepsilon|^{2} \ll\left(k_{\mathrm{B}} T\right)^{2} \\
\frac{a_{0} k_{\mathrm{B}}}{2 J} \frac{k_{\mathrm{F}}-\frac{1}{4}}{k_{\mathrm{F}}^{\prime}}\left[\phi_{0}^{\prime}\left(k_{\mathrm{F}}\right)-\phi_{0}^{\prime}\left(-k_{\mathrm{F}}\right)\right] T \\
\text { for }|\mu-\varepsilon| \ll\left(k_{\mathrm{B}} T\right)^{2} \wedge \varepsilon\left(k_{\mathrm{B}} T\right)^{2} \ll|\mu-\varepsilon| \wedge|\mu-\varepsilon|^{2} \ll\left(k_{\mathrm{B}} T\right)^{2} \\
\frac{a_{1} k_{\mathrm{B}}^{3}}{16 J^{3}} \frac{k_{\mathrm{F}}^{\prime \prime}}{k_{\mathrm{F}}^{\prime}}\left[\phi_{0}^{\prime}\left(k_{\mathrm{F}}\right)-\phi_{0}^{\prime}\left(-k_{\mathrm{F}}\right)\right] T^{3} \\
\text { for }|\mu-\varepsilon| \ll\left(k_{\mathrm{B}} T\right)^{2} \wedge|\mu-\varepsilon| \ll \varepsilon\left(k_{\mathrm{B}} T\right)^{2} \wedge|\mu-\varepsilon|^{2} \ll\left(k_{\mathrm{B}} T\right)^{2} .
\end{array}\right.
$$

This is equivalent to

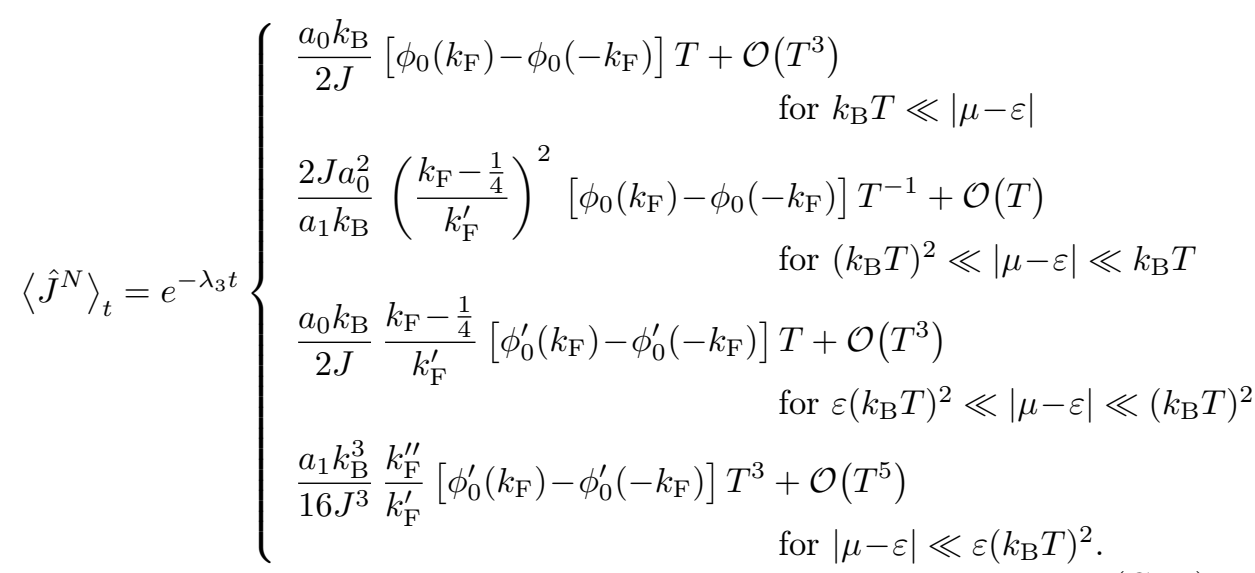

Plugging the formulas for $k_{\mathrm{F}}, k_{\mathrm{F}}^{\prime}$, and $k_{\mathrm{F}}^{\prime \prime}$ from App. C.3 into this equation, we obtain

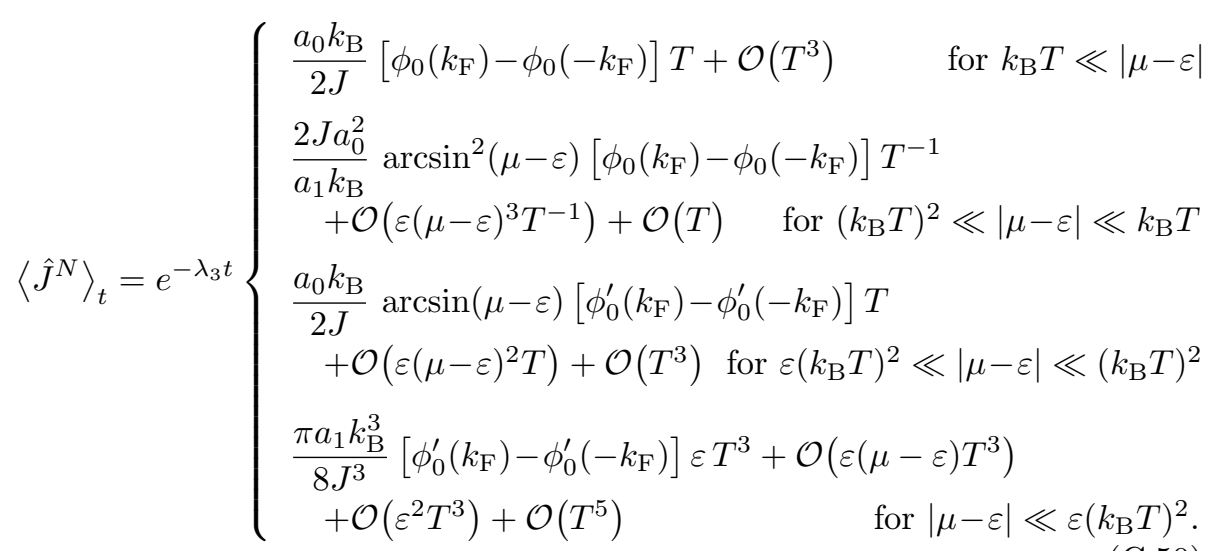

The same computation can be done for the expectation value of the total heat 
current $\hat{J}^{Q}$, but we will not present it here in all detail. From Eq. (3.128), we know that

$$
\left\langle\hat{J}^{Q}\right\rangle_{t}=\left\langle\hat{\Phi}\left[j^{Q}\right]\right\rangle_{t} \stackrel{(3.128)}{=} e^{-\lambda_{3} t} \frac{\left\langle\phi_{0}, \chi^{(3)}\right\rangle_{\mathrm{F}}\left\langle\chi^{(3)}, j^{Q}\right\rangle_{\mathrm{F}}}{\left\|\chi^{(3)}\right\|_{\mathrm{F}}^{2}} .
$$

Furthermore, use the Sommerfeld expansions of the occurring scalar products calculated in Eqs. (C.14), (C.16), and (C.18):

$$
\begin{aligned}
\left\langle\hat{J}^{Q}\right\rangle_{t}=e^{-\lambda_{3} t} & {\left[\frac{a_{0} k_{\mathrm{B}}}{2 J} k_{\mathrm{F}}^{\prime}\left[k_{\mathrm{F}}-\frac{1}{4}\right]\left[\phi_{0}\left(k_{\mathrm{F}}\right)-\phi_{0}\left(-k_{\mathrm{F}}\right)\right] T\right.} \\
& \left.+\frac{a_{1} k_{\mathrm{B}}^{3}}{8 J^{3}}\left(k_{\mathrm{F}}^{\prime}\right)^{2}\left[\phi_{0}^{\prime}\left(k_{\mathrm{F}}\right)+\phi_{0}^{\prime}\left(-k_{\mathrm{F}}\right)\right] T^{3}+\mathcal{O}\left(T^{5}\right)\right] \\
\times & \frac{\left[\frac{a_{1} k_{\mathrm{B}}^{3}}{16 J^{3}} k_{\mathrm{F}}^{\prime} T^{3}+\mathcal{O}\left(T^{5}\right)\right]}{\left[\frac{a_{0} k_{\mathrm{B}}}{2 J} k_{\mathrm{F}}^{\prime}\left[k_{\mathrm{F}}-\frac{1}{4}\right]^{2} T+\frac{a_{1} k_{\mathrm{B}}^{3}}{8 J^{3}}\left(k_{\mathrm{F}}^{\prime}\right)^{3} T^{3}+\mathcal{O}\left(T^{5}\right)\right]} .
\end{aligned}
$$

Now, we distinguish the different regimes that can occur. In these regimes, the expectation value of the heat current exhibits different leading order terms

$$
\left\langle\hat{J}^{Q}\right\rangle_{t}=e^{-\lambda_{3} t}\left\{\begin{array}{r}
\frac{a_{1} k_{\mathrm{B}}^{3}}{16 J^{3}} k_{\mathrm{F}}^{\prime} \frac{\phi_{0}\left(k_{\mathrm{F}}\right)-\phi_{0}\left(-k_{\mathrm{F}}\right)}{\left(k_{\mathrm{F}}-\frac{1}{4}\right)} T^{3}+\mathcal{O}\left(T^{5}\right) \\
\text { for }\left(k_{\mathrm{B}} T\right)^{2} \ll|\mu-\varepsilon| \wedge\left(k_{\mathrm{B}} T\right)^{2} \ll|\mu-\varepsilon|^{2} \\
\frac{a_{0} k_{\mathrm{B}}}{4 J} \frac{k_{\mathrm{F}}-\frac{1}{4}}{k_{\mathrm{F}}^{\prime}}\left[\phi_{0}\left(k_{\mathrm{F}}\right)-\phi_{0}\left(-k_{\mathrm{F}}\right)\right] T+\mathcal{O}\left(T^{3}\right) \\
\text { for }\left(k_{\mathrm{B}} T\right)^{2} \ll|\mu-\varepsilon| \wedge|\mu-\varepsilon|^{2} \ll\left(k_{\mathrm{B}} T\right)^{2} \\
\frac{a_{1} k_{\mathrm{B}}^{3}}{16 J^{3}}\left[\phi_{0}^{\prime}\left(k_{\mathrm{F}}\right)-\phi_{0}^{\prime}\left(-k_{\mathrm{F}}\right)\right] T^{3}+\mathcal{O}\left(T^{5}\right) \\
\text { for }|\mu-\varepsilon| \ll\left(k_{\mathrm{B}} T\right)^{2} \wedge|\mu-\varepsilon|^{2} \ll\left(k_{\mathrm{B}} T\right)^{2} .
\end{array}\right.
$$

This is equivalent to

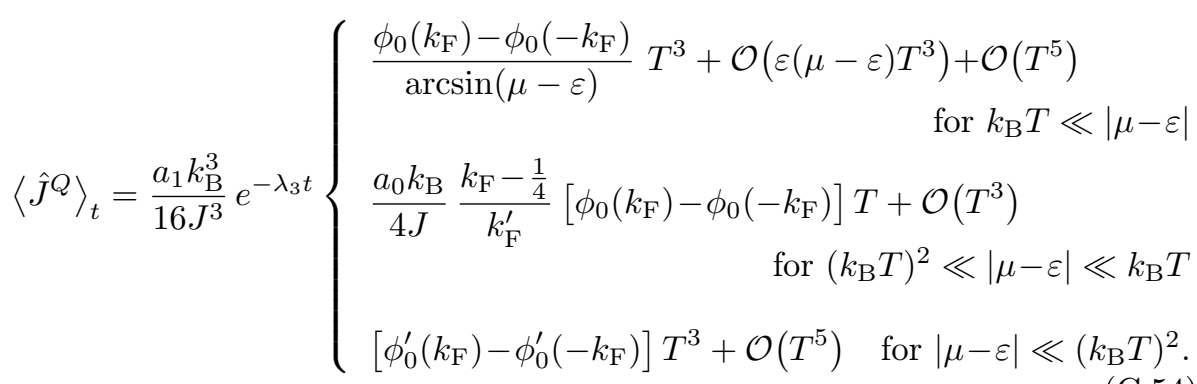

The last step is plugging the formulas for $k_{\mathrm{F}}, k_{\mathrm{F}}^{\prime}$, and $k_{\mathrm{F}}^{\prime \prime}$ from App. C.3 into 
this equation:

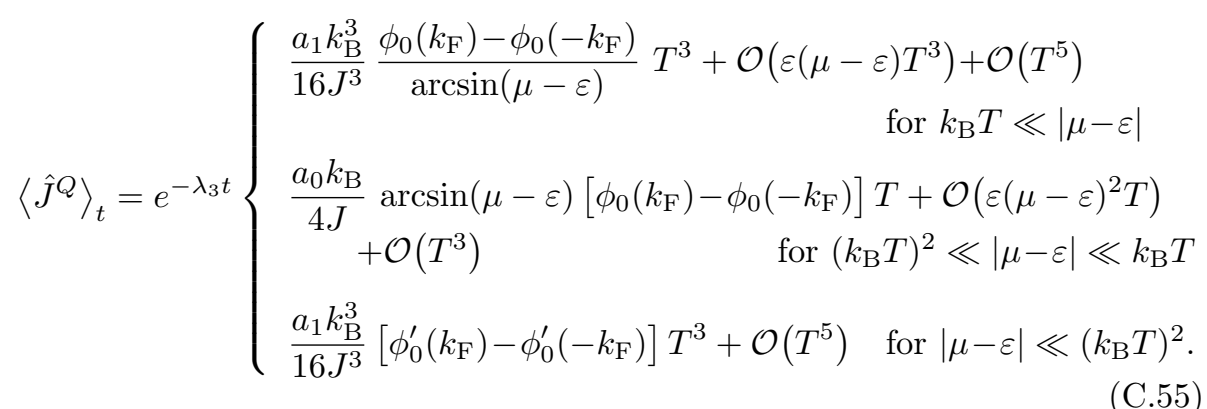

\section{C.6.2 Exemplary initial perturbations}

This section is devoted to calculating the currents which are present in the two exemplary initial perturbations $\phi_{0}(k)$ from Sec. 3.2.5.

Example 1: The first example is $\phi_{0}(k)=-(\beta \eta E / 2 J) j^{N}(k)$, see Eq. (3.80). As a start, the electrical conductivity $\sigma_{0}$ is calculated. We find it by considering the particle current of the initial perturbation:

$$
\left\langle\hat{J}^{N}\right\rangle_{t}=\left\langle j^{N}, \phi_{0}\right\rangle_{\mathrm{F}}=\sigma_{0} E
$$

with

$$
\begin{aligned}
\sigma_{0} & =2 J \beta \eta\left\langle j^{N}, j^{N}\right\rangle_{\mathrm{F}} \\
& =\left.2 J \beta \eta \sum_{n=0}^{\infty}\left(\frac{k_{\mathrm{B}} T}{2 J}\right)^{2 n+1} \frac{a_{n}}{(2 n) !} \frac{d^{2 n}}{d \tilde{\omega}^{2 n}} 2 k^{\prime}(\tilde{\omega})\left[\omega^{\prime}(k(\tilde{\omega}))\right]^{2}\right|_{\tilde{\omega}=\mu}+\mathcal{O}\left(e^{-\beta}\right) .
\end{aligned}
$$

Next, we make use of $1=\omega^{\prime}(k(\tilde{\omega})) k^{\prime}(\tilde{\omega})$ :

$$
\begin{aligned}
\sigma_{0} & =\left.2 J \beta \eta \sum_{n=0}^{\infty}\left(\frac{k_{\mathrm{B}} T}{2 J}\right)^{2 n+1} \frac{a_{n}}{(2 n) !} \frac{d^{2 n}}{d \tilde{\omega}^{2 n}} 2 \omega^{\prime}(k(\tilde{\omega}))\right|_{\tilde{\omega}=\mu}+\mathcal{O}\left(e^{-\beta}\right) \\
& =4 J a_{0} \eta \frac{1}{k_{\mathrm{F}}^{\prime}}+\mathcal{O}\left(T^{3}\right) .
\end{aligned}
$$

Now, we plug in the equation for $k_{\mathrm{F}}^{\prime}$ from App. C.3 and obtain

$$
\sigma_{0}=8 \pi a_{0} J \eta E \sqrt{1-(\mu-\varepsilon)^{2}}+\mathcal{O}(\varepsilon(\mu-\varepsilon))+\mathcal{O}\left(T^{2}\right) .
$$

After a time much larger than $1 / \lambda_{4}$, the particle current decays to

$$
\left\langle\hat{J}^{N}\right\rangle_{t}=e^{-\lambda_{3} t} \beta \eta E \frac{\left\langle\chi^{(3)}, j^{N}\right\rangle_{\mathrm{F}}^{2}}{\left\|\chi^{(3)}\right\|_{\mathrm{F}}^{2}}
$$


Using Eqs. (C.12) and (C.16), one obtains

$$
\left\langle\hat{J}^{N}\right\rangle_{t}=e^{-\lambda_{3} t} \beta \eta E \frac{\left[\frac{a_{0} k_{\mathrm{B}}}{2 J}\left(k_{\mathrm{F}}-\frac{1}{4}\right) T+\frac{a_{1} k_{\mathrm{B}}^{3}}{16 J^{3}} k_{\mathrm{F}}^{\prime \prime} T^{3}+\mathcal{O}\left(T^{5}\right)\right]^{2}}{\left[\frac{a_{0} k_{\mathrm{B}}}{2 J} k_{\mathrm{F}}^{\prime}\left[k_{\mathrm{F}}-\frac{1}{4}\right]^{2} T+\frac{a_{1} k_{\mathrm{B}}^{3}}{8 J^{3}}\left(k_{\mathrm{F}}^{\prime}\right)^{3} T^{3}+\mathcal{O}\left(T^{5}\right)\right]} .
$$

Furthermore, we get the leading terms of the different temperature regimes

$$
\left\langle\hat{J}^{N}\right\rangle_{t}=e^{-\lambda_{3} t} \beta \eta E\left\{\begin{array}{c}
\frac{a_{0} k_{\mathrm{B}}}{2 J} \frac{1}{k_{\mathrm{F}}^{\prime}} T+\mathcal{O}\left(T^{3}\right) \\
\text { for } \varepsilon\left(k_{\mathrm{B}} T\right)^{2} \ll|\mu-\varepsilon| \wedge\left(k_{\mathrm{B}} T\right)^{2} \ll|\mu-\varepsilon|^{2} \\
\frac{2 J a_{0}^{2}}{a_{1} k_{\mathrm{B}}} \frac{\left(k_{\mathrm{F}}-\frac{1}{4}\right)^{2}}{\left(k_{\mathrm{F}}^{\prime}\right)^{3}} T^{-1}+\mathcal{O}(T) \\
\text { for } \varepsilon\left(k_{\mathrm{B}} T\right)^{2} \ll|\mu-\varepsilon| \wedge|\mu-\varepsilon|^{2} \ll\left(k_{\mathrm{B}} T\right)^{2} \\
\frac{a_{1} k_{\mathrm{B}}^{3}}{16 J^{3}} \frac{\left(k_{\mathrm{F}}^{\prime \prime}\right)^{2}}{\left(k_{\mathrm{F}}^{\prime}\right)^{3}} T^{3}+\mathcal{O}\left(T^{5}\right) \\
\text { for }|\mu-\varepsilon| \ll \varepsilon\left(k_{\mathrm{B}} T\right)^{2} \wedge|\mu-\varepsilon|^{2} \ll\left(k_{\mathrm{B}} T\right)^{2} .
\end{array}\right.
$$

This can be simplified to

$$
\left\langle\hat{J}^{N}\right\rangle_{t}=e^{-\lambda_{3} t} \eta E\left\{\begin{array}{lr}
a_{0} \frac{1}{k_{\mathrm{F}}^{\prime}}+\mathcal{O}\left(T^{2}\right) & \text { for } k_{\mathrm{B}} T \ll|\mu-\varepsilon| \\
\frac{4 J^{2} a_{0}^{2}}{a_{1} k_{\mathrm{B}}^{2}} \frac{\left(k_{\mathrm{F}}-\frac{1}{4}\right)^{2}}{\left(k_{\mathrm{F}}^{\prime}\right)^{3}} T^{-2}+\mathcal{O}\left(T^{0}\right) & \text { for } \varepsilon\left(k_{\mathrm{B}} T\right)^{2} \ll|\mu-\varepsilon| \ll k_{\mathrm{B}} T \\
\frac{a_{1} k_{\mathrm{B}}^{2}}{8 J^{2}} \frac{\left(k_{\mathrm{F}}^{\prime \prime}\right)^{2}}{\left(k_{\mathrm{F}}^{\prime}\right)^{3}} T^{2}+\mathcal{O}\left(T^{4}\right) & \text { for }|\mu-\varepsilon| \ll \varepsilon\left(k_{\mathrm{B}} T\right)^{2} .
\end{array}\right.
$$

Plugging the formulas for $k_{\mathrm{F}}, k_{\mathrm{F}}^{\prime}$, and $k_{\mathrm{F}}^{\prime \prime}$ from App. C.3 into this equation, leads to

$$
\left\langle\hat{J}^{N}\right\rangle_{t}=e^{-\lambda_{3} t} \eta E\left\{\begin{array}{rr}
\frac{2 \pi a_{0}}{\sqrt{1-(\mu-\varepsilon)^{2}}}+\mathcal{O}\left(\varepsilon T^{0}\right)+\mathcal{O}\left(T^{2}\right) & \text { for } k_{\mathrm{B}} T \ll|\mu-\varepsilon| \\
\frac{8 \pi J^{2} a_{0}^{2}}{a_{1} k_{\mathrm{B}}^{2}} \arcsin (\mu-\varepsilon)^{2} T^{-2}+\mathcal{O}\left(\varepsilon^{4} T^{3}\right)+\mathcal{O}\left(T^{0}\right) & \text { for } \varepsilon\left(k_{\mathrm{B}} T\right)^{2} \ll|\mu-\varepsilon| \ll k_{\mathrm{B}} T \\
\frac{4 \pi a_{1} k_{\mathrm{B}}^{2}}{J^{2}} \varepsilon^{2} T^{2}+\mathcal{O}\left(\varepsilon(\mu-\varepsilon)^{3} T^{3}\right)+\mathcal{O}\left(T^{4}\right) \\
\text { for }|\mu-\varepsilon| \ll \varepsilon\left(k_{\mathrm{B}} T\right)^{2} .
\end{array}\right.
$$

Now, we consider the total heat current. For $t \ll 1 / \lambda_{4}$, its expectation value becomes

$$
\left\langle\hat{J}^{Q}\right\rangle_{t}=e^{-\lambda_{3} t} \beta \eta E \frac{\left\langle j^{N}, \chi^{(3)}\right\rangle_{\mathrm{F}}\left\langle\chi^{(3)}, j^{Q}\right\rangle_{\mathrm{F}}}{\left\|\chi^{(3)}\right\|_{\mathrm{F}}^{2}} .
$$


Using Eqs. (C.12), (C.14), and (C.16), we find

$$
\left\langle\hat{J}^{Q}\right\rangle_{t}=e^{-\lambda_{3} t} \beta \eta E \frac{\left[\frac{a_{0} k_{\mathrm{B}}}{2 J}\left(k_{\mathrm{F}}-\frac{1}{4}\right) T+\frac{a_{1} k_{\mathrm{B}}^{3}}{16 J^{3}} k_{\mathrm{F}}^{\prime \prime} T^{3}+\mathcal{O}\left(T^{5}\right)\right]\left[\frac{a_{1} k_{\mathrm{B}}^{3}}{16 J^{3}} k_{\mathrm{F}}^{\prime} T^{3}+\mathcal{O}\left(T^{5}\right)\right]}{\left[\frac{a_{0} k_{\mathrm{B}}}{2 J} k_{\mathrm{F}}^{\prime}\left[k_{\mathrm{F}}-\frac{1}{4}\right]^{2} T+\frac{a_{1} k_{\mathrm{B}}^{3}}{8 J^{3}}\left(k_{\mathrm{F}}^{\prime}\right)^{3} T^{3}+\mathcal{O}\left(T^{5}\right)\right]} .
$$

Furthermore, the leading terms of the different temperature regimes is obtained:

$$
\left\langle\hat{J}^{Q}\right\rangle_{t}=e^{-\lambda_{3} t} \beta \eta E\left\{\begin{array}{lr}
\frac{a_{1} k_{\mathrm{B}}^{3}}{16 J^{3}} \frac{1}{k_{\mathrm{F}}-\frac{1}{4}} T^{3}+\mathcal{O}\left(T^{5}\right) & \text { for } k_{\mathrm{B}} T \ll|\mu-\varepsilon| \\
\frac{a_{0} k_{\mathrm{B}}}{4 J} \frac{k_{\mathrm{F}}-\frac{1}{4}}{\left(k_{\mathrm{F}}^{\prime}\right)^{2}} T+\mathcal{O}\left(T^{3}\right) & \text { for } \varepsilon\left(k_{\mathrm{B}} T\right)^{2} \ll|\mu-\varepsilon| \ll k_{\mathrm{B}} T \\
\frac{a_{1} k_{\mathrm{B}}^{3}}{32 J^{3}} \frac{k_{\mathrm{F}}^{\prime \prime}}{\left(k_{\mathrm{F}}^{\prime}\right)^{2}} T^{3}+\mathcal{O}\left(T^{5}\right) & \text { for }|\mu-\varepsilon| \ll \varepsilon\left(k_{\mathrm{B}} T\right)^{2} .
\end{array}\right.
$$

Plugging the formulas for $k_{\mathrm{F}}, k_{\mathrm{F}}^{\prime}$, and $k_{\mathrm{F}}^{\prime \prime}$ from App. C.3 into this equation, we get

$$
\left\langle\hat{J}^{Q}\right\rangle_{t}=e^{-\lambda_{3} t} \eta E\left\{\begin{array}{c}
\frac{\pi a_{1} k_{\mathrm{B}}^{2}}{4 J^{2}} \frac{1}{\arcsin (\mu-\varepsilon)} T^{2}+\mathcal{O}\left(\varepsilon T^{2}\right)+\mathcal{O}\left(T^{4}\right) \\
\quad \text { for } k_{\mathrm{B}} T \ll|\mu-\varepsilon| \\
2 \pi a_{0} \arcsin (\mu-\varepsilon)+\mathcal{O}\left(\varepsilon(\mu-\varepsilon)^{2} T^{0}\right)+\mathcal{O}\left(T^{2}\right) \\
\text { for } \varepsilon\left(k_{\mathrm{B}} T\right)^{2} \ll|\mu-\varepsilon| \ll k_{\mathrm{B}} T \\
\frac{\pi^{3} a_{1} k_{\mathrm{B}}^{3}}{8 J^{2}} \varepsilon T^{2}+\mathcal{O}\left(\varepsilon^{2} T^{2}\right)+\mathcal{O}\left(T^{4}\right) \quad \text { for }|\mu-\varepsilon| \ll \varepsilon\left(k_{\mathrm{B}} T\right)^{2} .
\end{array}\right.
$$

Example 2: The second example is $\phi_{0}(k)=\beta^{2} \Delta T \operatorname{sgn}(k)(\omega(k)-\mu)$, see Eq. (3.84). From this example, we can calculate the thermal conductivity $\kappa_{0}$, because of

$$
\left\langle\hat{J}^{Q}\right\rangle_{t}=\left\langle j^{Q}, \phi_{0}\right\rangle_{\mathrm{F}}=\kappa_{0} \Delta T .
$$

Thus, the thermal conductivity is

$$
\begin{aligned}
\kappa_{0}= & 4 J^{2} \beta^{2}\left\langle j^{Q}, \operatorname{sgn} \cdot(\omega-\mu)\right\rangle_{\mathrm{F}} \\
= & 4 J^{2} \beta^{2} \sum_{n=0}^{\infty}\left(\frac{k_{\mathrm{B}} T}{2 J}\right)^{2 n+1} \frac{a_{n}}{(2 n) !} \\
& \quad \times\left.\frac{d^{2 n}}{d \tilde{\omega}^{2 n}} 2 k^{\prime}(\tilde{\omega}) \omega^{\prime}(k(\tilde{\omega}))[\omega(k(\tilde{\omega}))-\mu]^{2} \operatorname{sgn}(k(\tilde{\omega}))\right|_{\tilde{\omega}=\mu}+\mathcal{O}\left(e^{-\beta}\right) .
\end{aligned}
$$

Now, we use $\omega^{\prime}(k(\tilde{\omega})) k^{\prime}(\tilde{\omega})=1$ and the fact that $\operatorname{sgn}(k)=1$ for $k \in(0,1 / 2)$ :

$$
\kappa_{0}=\left.8 J^{2} \sum_{n=0}^{\infty}\left(\frac{k_{\mathrm{B}} T}{2 J}\right)^{2 n-1} \frac{a_{n}}{(2 n) !} \frac{d^{2 n}}{d \tilde{\omega}^{2 n}}(\tilde{\omega}-\mu)^{2}\right|_{\tilde{\omega}=\mu}+\mathcal{O}\left(e^{-\beta}\right) .
$$


The only series coefficient that is not zero is the one for $n=1$. Thence, one gets

$$
\begin{gathered}
\kappa_{0}=4 J^{2} \beta^{2}\left(\frac{k_{\mathrm{B}} T}{2 J}\right)^{3} \frac{a_{1}}{2} 4+\mathcal{O}\left(e^{-\beta}\right) \\
\Rightarrow \kappa_{0}=4 J a_{1} k_{\mathrm{B}} T \Delta T+\mathcal{O}\left(e^{-\beta}\right) .
\end{gathered}
$$

For $t \gg 1 / \lambda_{4}$, the particle current is

$$
\left\langle\hat{J}^{N}\right\rangle_{t}=e^{-\lambda_{3} t} \beta^{2} \Delta T \frac{\left\langle\operatorname{sgn}(\omega-\mu), \chi^{(3)}\right\rangle_{\mathrm{F}}\left\langle\chi^{(3)}, j^{N}\right\rangle_{\mathrm{F}}}{\left\|\chi^{(3)}\right\|_{\mathrm{F}}^{2}} .
$$

Using Eqs. (C.12), (C.16), and (C.20), we find

$$
\begin{aligned}
\left\langle\hat{J}^{N}\right\rangle_{t}=e^{-\lambda_{3} t} \beta^{2} \Delta T\left[\frac{a_{1} k_{\mathrm{B}}^{3}}{8 J^{3}}\left[\left(k_{\mathrm{F}}^{\prime}\right)^{2}+k_{\mathrm{F}}^{\prime \prime}\left(k_{\mathrm{F}}-\frac{1}{4}\right)\right] T^{3}+\mathcal{O}\left(T^{5}\right)\right] \\
\times \frac{\left[\frac{a_{0} k_{\mathrm{B}}}{2 J}\left(k_{\mathrm{F}}-\frac{1}{4}\right) T+\frac{a_{1} k_{\mathrm{B}}^{3}}{16 J^{3}} k_{\mathrm{F}}^{\prime \prime} T^{3}+\mathcal{O}\left(T^{5}\right)\right]}{\left[\frac{a_{0} k_{\mathrm{B}}}{2 J} k_{\mathrm{F}}^{\prime}\left[k_{\mathrm{F}}-\frac{1}{4}\right]^{2} T+\frac{a_{1} k_{\mathrm{B}}^{3}}{8 J^{3}}\left(k_{\mathrm{F}}^{\prime}\right)^{3} T^{3}+\mathcal{O}\left(T^{5}\right)\right]} .
\end{aligned}
$$

Furthermore, we obtain the leading terms of the different temperature regimes

$$
\left\langle\hat{J}^{N}\right\rangle_{t}=e^{-\lambda_{3} t} \beta^{2} \Delta T\left\{\begin{array}{c}
\frac{a_{1} k_{\mathrm{B}}^{3}}{8 J^{3}} \frac{\left(k_{\mathrm{F}}^{\prime}\right)^{2}+k_{\mathrm{F}}^{\prime \prime}\left(k_{\mathrm{F}}-\frac{1}{4}\right)}{k_{\mathrm{F}}^{\prime}\left(k_{\mathrm{F}}-\frac{1}{4}\right)} T^{3}+\mathcal{O}\left(T^{5}\right) \\
\text { for } k_{\mathrm{B}} T \ll|\mu-\varepsilon| \\
\frac{a_{0} k_{\mathrm{B}}}{2 J} \frac{\left[\left(k_{\mathrm{F}}^{\prime}\right)^{2}+k_{\mathrm{F}}^{\prime \prime}\left(k_{\mathrm{F}}-\frac{1}{4}\right)\right]\left(k_{\mathrm{F}}-\frac{1}{4}\right)}{\left(k_{\mathrm{F}}^{\prime}\right)^{3}} T+\mathcal{O}\left(T^{3}\right) \\
\text { for } \varepsilon\left(k_{\mathrm{B}} T\right)^{2} \ll|\mu-\varepsilon| \ll k_{\mathrm{B}} T \\
\frac{a_{1} k_{\mathrm{B}}^{3}}{16 J^{3}} \frac{\left[\left(k_{\mathrm{F}}^{\prime}\right)^{2}+k_{\mathrm{F}}^{\prime \prime}\left(k_{\mathrm{F}}-\frac{1}{4}\right)\right] k_{\mathrm{F}}^{\prime \prime}}{\left(k_{\mathrm{F}}^{\prime}\right)^{3}} T^{3}+\mathcal{O}\left(T^{5}\right) \\
\text { for }|\mu-\varepsilon| \ll \varepsilon\left(k_{\mathrm{B}} T\right)^{2} .
\end{array}\right.
$$

This leads to

$$
\left\langle\hat{J}^{N}\right\rangle_{t}=e^{-\lambda_{3} t} \Delta T\left\{\begin{array}{lr}
\frac{a_{1} k_{\mathrm{B}}}{2 J} \frac{\left(k_{\mathrm{F}}^{\prime}\right)^{2}+k_{\mathrm{F}}^{\prime \prime}\left(k_{\mathrm{F}}-\frac{1}{4}\right)}{k_{\mathrm{F}}^{\prime}\left(k_{\mathrm{F}}-\frac{1}{4}\right)} T+\mathcal{O}\left(T^{3}\right) & \text { for } k_{\mathrm{B}} T \ll|\mu-\varepsilon| \\
\frac{2 J a_{0}}{k_{\mathrm{B}}} \frac{k_{\mathrm{F}}-\frac{1}{4}}{k_{\mathrm{F}}^{\prime}} T^{-1}+\mathcal{O}(T) & \text { for } \varepsilon\left(k_{\mathrm{B}} T\right)^{2} \ll|\mu-\varepsilon| \ll k_{\mathrm{B}} T \\
\frac{a_{1} k_{\mathrm{B}}}{4 J} \frac{k_{\mathrm{F}}^{\prime \prime}}{k_{\mathrm{F}}^{\prime}} T+\mathcal{O}\left(T^{3}\right) & \text { for }|\mu-\varepsilon| \ll \varepsilon\left(k_{\mathrm{B}} T\right)^{2} .
\end{array}\right.
$$

Plugging the formulas for $k_{\mathrm{F}}, k_{\mathrm{F}}^{\prime}$, and $k_{\mathrm{F}}^{\prime \prime}$ from App. C.3 into this equation, we 
obtain

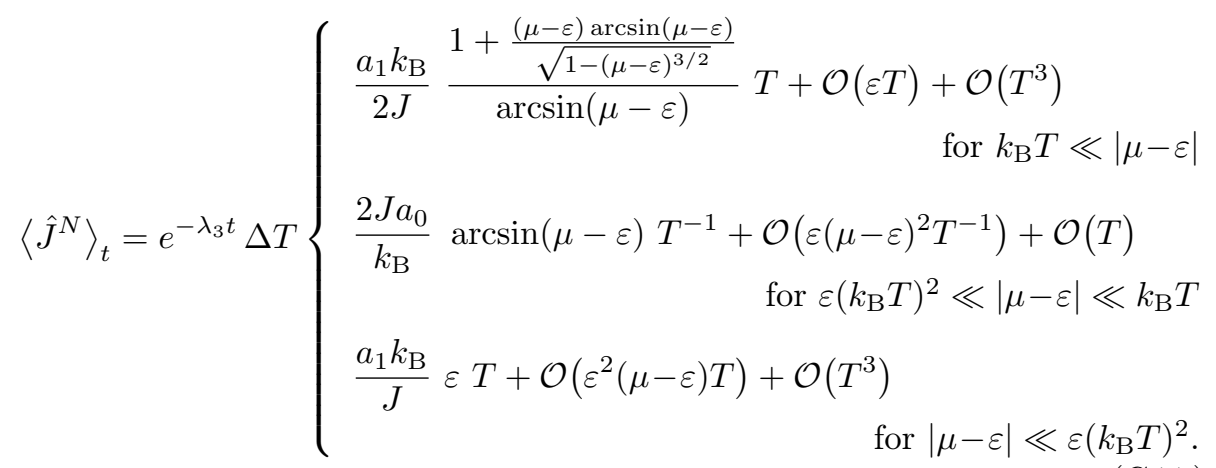

Now, we consider the total heat current. For long times, which are much longer than $1 / \lambda_{4}$, one gets

$$
\left\langle\hat{J}^{Q}\right\rangle_{t}=e^{-\lambda_{3} t} \beta^{2} \Delta T \frac{\left\langle\operatorname{sgn}(\omega-\mu), \chi^{(3)}\right\rangle_{\mathrm{F}}\left\langle\chi^{(3)}, j^{Q}\right\rangle_{\mathrm{F}}}{\left\|\chi^{(3)}\right\|_{\mathrm{F}}^{2}} .
$$

Using Eqs. (C.14), (C.16), and (C.20) leads to

$$
\begin{aligned}
& \left\langle\hat{J}^{Q}\right\rangle_{t}= \\
& =e^{-\lambda_{3} t} \beta^{2} \Delta T \frac{\left[\frac{a_{1} k_{\mathrm{B}}^{3}}{8 J^{3}}\left[\left(k_{\mathrm{F}}^{\prime}\right)^{2}+k_{\mathrm{F}}^{\prime \prime}\left(k_{\mathrm{F}}-\frac{1}{4}\right)\right] T^{3}+\mathcal{O}\left(T^{5}\right)\right]\left[\frac{a_{1} k_{\mathrm{B}}^{3}}{16 J^{3}} k_{\mathrm{F}}^{\prime} T^{3}+\mathcal{O}\left(T^{5}\right)\right]}{\frac{a_{0} k_{\mathrm{B}}}{2 J} k_{\mathrm{F}}^{\prime}\left[k_{\mathrm{F}}-\frac{1}{4}\right]^{2} T+\frac{a_{1} k_{\mathrm{B}}^{3}}{8 J^{3}}\left(k_{\mathrm{F}}^{\prime}\right)^{3} T^{3}+\mathcal{O}\left(T^{5}\right)} \\
& =e^{-\lambda_{3} t} \beta^{2} \Delta T \frac{\frac{1}{2}\left(\frac{a_{1} k_{\mathrm{B}}^{3}}{8 J^{3}}\right)^{2} k_{\mathrm{F}}^{\prime}\left[\left(k_{\mathrm{F}}^{\prime}\right)^{2}+k_{\mathrm{F}}^{\prime \prime}\left(k_{\mathrm{F}}-\frac{1}{4}\right)\right] T^{6}+\mathcal{O}\left(T^{8}\right)}{\frac{a_{0} k_{\mathrm{B}}}{2 J} k_{\mathrm{F}}^{\prime}\left[k_{\mathrm{F}}-\frac{1}{4}\right]^{2} T+\frac{a_{1} k_{\mathrm{B}}^{3}}{8 J^{3}}\left(k_{\mathrm{F}}^{\prime}\right)^{3} T^{3}+\mathcal{O}\left(T^{5}\right)} .
\end{aligned}
$$

Furthermore, we obtain the leading terms of the different temperature regimes

$$
\left\langle\hat{J}^{Q}\right\rangle_{t}=e^{-\lambda_{3} t} \beta^{2} \Delta T\left\{\begin{array}{cc}
\frac{a_{1}^{2} k_{\mathrm{B}}^{5}}{64 a_{0} J^{5}} k_{\mathrm{F}}^{\prime}\left[\left(k_{\mathrm{F}}^{\prime}\right)^{2}+k_{\mathrm{F}}^{\prime \prime}\left(k_{\mathrm{F}}-\frac{1}{4}\right)\right] T^{5}+\mathcal{O}\left(T^{7}\right) & \text { for } k_{\mathrm{B}} T \ll|\mu-\varepsilon| \\
\frac{a_{1} k_{\mathrm{B}}^{3}}{16 J^{3}} \frac{\left(k_{\mathrm{F}}^{\prime}\right)^{2}+k_{\mathrm{F}}^{\prime \prime}\left(k_{\mathrm{F}}-\frac{1}{4}\right)}{\left(k_{\mathrm{F}}^{\prime}\right)^{2}} T^{3}+\mathcal{O}\left(T^{5}\right) & \text { for }|\mu-\varepsilon| \ll k_{\mathrm{B}} T .
\end{array}\right.
$$

The fact that $k_{\mathrm{F}}-\frac{1}{4}=\mathcal{O}(\arcsin (\mu-\varepsilon))$ leads to

$$
\left\langle\hat{J}^{Q}\right\rangle_{t}=e^{-\lambda_{3} t} \Delta T\left\{\begin{array}{rr}
\frac{a_{1}^{2} k_{\mathrm{B}}^{3}}{16 a_{0} J^{3}} k_{\mathrm{F}}^{\prime}\left[\left(k_{\mathrm{F}}^{\prime}\right)^{2}+k_{\mathrm{F}}^{\prime \prime}\left(k_{\mathrm{F}}-\frac{1}{4}\right)\right] T^{3}+\mathcal{O}\left(T^{5}\right) \\
\text { for } k_{\mathrm{B}} T \ll|\mu-\varepsilon| \\
\frac{a_{1} k_{\mathrm{B}}}{4 J} T+\mathcal{O}((\mu-\varepsilon) T)+\mathcal{O}\left(T^{3}\right) & \text { for }|\mu-\varepsilon| \ll k_{\mathrm{B}} T . \\
\text { (C.81) }
\end{array}\right.
$$

Plugging the formulas for $k_{\mathrm{F}}, k_{\mathrm{F}}^{\prime}$, and $k_{\mathrm{F}}^{\prime \prime}$ from App. C.3 into this equation, we 
obtain

$$
\left\langle\hat{J}^{Q}\right\rangle_{t}=e^{-\lambda_{3} t} \Delta T\left\{\begin{array}{cc}
\frac{a_{1}^{2} k_{\mathrm{B}}^{3} T^{3}}{128 \pi^{3} a_{0} J^{3} \sqrt{1-(\mu-\varepsilon)^{2}}}\left[\frac{1}{1-(\mu-\varepsilon)^{2}}+\frac{\arcsin (\mu-\varepsilon)}{\sqrt{1-(\mu-\varepsilon)^{3 / 2}}}\right] \\
+\mathcal{O}\left(T^{5}\right) & \text { for } k_{\mathrm{B}} T \ll|\mu-\varepsilon| \\
\frac{a_{1} k_{\mathrm{B}}}{4 J} T+\mathcal{O}((\mu-\varepsilon) T)+\mathcal{O}\left(T^{3}\right) & \text { for }|\mu-\varepsilon| \ll k_{\mathrm{B}} T .
\end{array}\right.
$$

\section{C.7 Fixed-point Fermi-Dirac distribution}

For any dispersion relation $\omega(k)$, the collision term becomes zero when the Fermi-Dirac distribution is plugged in:

$$
\begin{aligned}
\mathcal{I}_{\text {coll }}[f]= & \int d k_{2} d k_{3} d k_{4} \sum_{m=-1}^{1} \delta\left(\Delta k_{\vec{k}}+m\right) \delta\left(\Delta \omega_{\vec{k}}\right) \\
& \times\left[\left(1-f_{1}\right)\left(1-f_{2}\right) f_{3} f_{4}-f_{1} f_{2}\left(1-f_{3}\right)\left(1-f_{4}\right)\right]
\end{aligned}
$$

It becomes zero, because the last factor $\left(1-f_{1}\right)\left(1-f_{2}\right) f_{3} f_{4}-f_{1} f_{2}\left(1-f_{3}\right)\left(1-f_{4}\right)$ vanishes. The first term of this factor is compensated by the second due to energy conservation:

$$
\begin{aligned}
& \left(1-f_{1}\right)\left(1-f_{2}\right) f_{3} f_{4} \\
& =\frac{1}{e^{-\beta\left(\omega_{1}-\mu\right)}+1} \frac{1}{e^{-\beta\left(\omega_{2}-\mu\right)}+1} \frac{1}{e^{\beta\left(\omega_{3}-\mu\right)}+1} \frac{1}{e^{\beta\left(\omega_{4}-\mu\right)}+1} \\
& =\frac{e^{\beta\left(\omega_{1}-\mu\right)}}{e^{\beta\left(\omega_{1}-\mu\right)}+1} \frac{e^{\beta\left(\omega_{2}-\mu\right)}}{e^{\beta\left(\omega_{2}-\mu\right)}+1} \frac{e^{-\beta\left(\omega_{3}-\mu\right)}}{e^{-\beta\left(\omega_{3}-\mu\right)}+1} \frac{e^{-\beta\left(\omega_{4}-\mu\right)}}{e^{-\beta\left(\omega_{4}-\mu\right)}+1} \\
& =f_{1} f_{2}\left(1-f_{3}\right)\left(1-f_{4}\right) e^{\beta\left(\omega_{1}+\omega_{2}-\omega_{3}-\omega_{4}\right)} .
\end{aligned}
$$

In the last line, the exponential factor is one due to energy conservation $\Delta \omega_{\vec{k}}=$ $\omega_{1}+\omega_{2}-\omega_{3}-\omega_{4}=0$. Thus, we find

$$
\delta\left(\Delta \omega_{\vec{k}}\right)\left[\left(1-f_{1}\right)\left(1-f_{2}\right) f_{3} f_{4}-f_{1} f_{2}\left(1-f_{3}\right)\left(1-f_{4}\right)\right] .
$$

Therefore, one obtains

$$
\mathcal{I}_{\text {coll }}[f]=0 \quad \forall \omega .
$$

\section{C.8 Stationary states for nearest-neighbor hop- ping}

In this section, we consider the stationary states $f^{S}$ for the case of vanishing next-to-nearest-neighbor hopping, i.e. $\varepsilon=0$. These states were described by Fürst et al. (2012) and introduced in Eq. (3.19). We will show that the collision term vanishes for $f^{S}$. 
Plugging $f^{S}$ into the collision term leads to

$$
\begin{aligned}
\mathcal{I}_{\text {coll }}^{\varepsilon=0}\left[f^{S}\right]_{1}= & \int d k_{2} d k_{3} d k_{4} \sum_{m=-1}^{1} \delta\left(\Delta k_{\vec{k}}+m\right) \delta\left(\Delta \omega_{\vec{k}}^{\varepsilon=0}\right) \\
& \times\left[\left(1-f_{1}^{S}\right)\left(1-f_{2}^{S}\right) f_{3}^{S} f_{4}^{S}-f_{1}^{S} f_{2}^{S}\left(1-f_{3}^{S}\right)\left(1-f_{4}^{S}\right)\right] \\
= & \int_{-1 / 2}^{1 / 2} d k_{3}\left[\left(1-f_{1}^{S}\right)\left(1-\bar{f}_{1}^{S}\right) f_{3}^{S} \bar{f}_{3}^{S}-f_{1}^{S} \bar{f}_{1}^{S}\left(1-f_{3}^{S}\right)\left(1-\bar{f}_{3}^{S}\right)\right],
\end{aligned}
$$

with $\bar{f}_{j}^{S}:=f^{S}\left(\frac{1}{2}-k_{j}\right)$. As in App. C.7, where we showed that the Fermi-Dirac distribution is a fixed point of the collision term, we will show that the factor $\left(1-f_{1}^{S}\right)\left(1-\bar{f}_{1}^{S}\right) f_{3}^{S} \bar{f}_{3}^{S}-f_{1}^{S} \bar{f}_{1}^{S}\left(1-f_{3}^{S}\right)\left(1-\bar{f}_{3}^{S}\right)$ vanishes:

$$
\begin{aligned}
\left(1-f_{1}^{S}\right)\left(1-\bar{f}_{1}^{S}\right) f_{3}^{S} \bar{f}_{3}^{S}-f_{1}^{S} \bar{f}_{1}^{S}\left(1-f_{3}^{S}\right)\left(1-\bar{f}_{3}^{S}\right) & \\
= & \frac{1}{e^{-\phi_{1}^{S}+a}+1} \frac{1}{e^{\phi_{1}^{S}+a}+1} \frac{1}{e^{\phi_{3}^{S}-a}+1} \frac{1}{e^{-\phi_{3}^{S}-a}+1} \\
& \quad-\frac{1}{e^{\phi_{1}^{S}-a}+1} \frac{1}{e^{-\phi_{1}^{S}-a}+1} \frac{1}{e^{\phi_{3}^{S}+a}+1} \frac{1}{e^{\phi_{3}^{S}-a}+1} .
\end{aligned}
$$

Here, we used the symmetry of the stationary state, to be specific $\phi^{S}\left(\frac{1}{2}-k\right)=$ $-\phi^{S}(k)$, see Eq. (3.20). Now, the common factors of the two terms is pulled out:

$$
\begin{aligned}
& \left(1-f_{1}^{S}\right)\left(1-\bar{f}_{1}^{S}\right) f_{3}^{S} \bar{f}_{3}^{S}-f_{1}^{S} \bar{f}_{1}^{S}\left(1-f_{3}^{S}\right)\left(1-\bar{f}_{3}^{S}\right) \\
& =\frac{1}{e^{\phi_{1}^{S}-a}+1} \frac{1}{e^{\phi_{1}^{S}+a}+1} \frac{1}{e^{\phi_{3}^{S}-a}+1} \frac{1}{e^{\phi_{3}^{S}+a}+1} \underbrace{\left[e^{\phi_{1}^{S}-a} e^{\phi_{3}^{S}+a}-e^{\phi_{1}^{S}+a} e^{\phi_{3}^{S}-a}\right]}_{=e^{\phi_{1}^{S}+\phi_{3}^{S}}-e^{\phi_{1}^{S}+\phi_{3}^{S}}=0} .
\end{aligned}
$$

Then, we realize that the remains add up to zero independent of $k_{1}, k_{3}$, and the choice of $a \in \mathbb{R}$. Thus, indeed, the quasiparticle momentum distribution $f^{S}(k)$ is stationary for $\varepsilon=0$ :

$$
\mathcal{I}_{\text {coll }}^{\varepsilon=0}\left[f^{S}\right]=0 .
$$

\section{C.9 Further simplification of $\mathcal{L}_{m n}^{0}$}

In this part of the appendix, we record some simplifications that we found while calculating $\mathcal{L}_{m n}^{0}$. This operator is the $\varepsilon \rightarrow 0$ limit of $\mathcal{L}_{m n}^{\varepsilon}=\left\langle\zeta_{m}, \hat{\mathcal{L}}\left[\zeta_{n}\right]\right\rangle_{\mathrm{F}}$, which is defined in Eq. (3.151). In the following, we show how to get rid of the factor $\left|c_{p}-c_{q}\right|$ in the denominator of the integrand of $\mathcal{L}_{m n}^{0}$. This can be useful for numerical evaluations, because $\left|c_{p}-c_{q}\right|$ contains zeros that are eliminated by the denominator.

We start with $\mathcal{L}_{m n}^{0}$ given in Eq. (3.162) from Sec. 3.6.3:

$$
\begin{aligned}
\mathcal{L}_{m n}^{0}=- & \frac{U^{2}}{\hbar J} \vartheta_{m} \vartheta_{n} \Delta_{m+n} \int d p d q \frac{\left(c_{n p}-c_{n q}\right)\left(c_{m p}-c_{m q}\right)}{\left|c_{p}-c_{q}\right|} \\
& \left.\times\left[1-f^{0}\left(\frac{1}{4}+p\right)\right]\left[1-f^{0}\left(\frac{1}{4}\right]^{2}-p\right)\right] f^{0}\left(\frac{1}{4}+q\right) f^{0}\left(\frac{1}{4}-q\right),
\end{aligned}
$$


where $\vartheta_{n \geqslant 0}=1$ and $\vartheta_{n<0}=0$, which was defined in Eq. (3.150). As a first step, we perform the actual elimination of the zeros:

$$
\frac{c_{n p}-c_{n q}}{c_{p}-c_{q}}=\frac{c_{(n-2) p}-c_{(n-2) q}}{c_{p}-c_{q}}+2 c_{(n-1) p}+4 \sum_{l=1}^{n-2} c_{l p} c_{(n-1-l) q}+2 c_{(n-1) q}
$$

For example

$$
\begin{array}{ll}
n=1: & \frac{c_{p}-c_{q}}{c_{p}-c_{q}}=1 \\
n=2: & \frac{c_{2 p}-c_{2 q}}{c_{p}-c_{q}}=2\left(c_{p}+c_{q}\right) .
\end{array}
$$

We can also write this as

$$
\begin{aligned}
& \frac{c_{n p}-c_{n q}}{c_{p}-c_{q}}=J_{n}\left(\frac{p+q}{2}\right) J_{n}\left(\frac{p-q}{2}\right) \\
& J_{n}(x)=c_{n x} / c_{x}=\sum_{l=1}^{n-1} c_{(n-2 l) x} .
\end{aligned}
$$

As we see, the remaining expression is without divergence. Note that it only contains terms of the form $c_{j p} c_{m p}$ where $m+j \in\{n-1, n-3, n-5, \ldots\}$. We notice that in Eq. (3.162) most terms in the $p$ integration are products of cosines of $p$.

However, before we can use this, we need to separate $\left|c_{p}-c_{q}\right|=\left(c_{p}-c_{q}\right) \operatorname{sgn}\left(c_{p}-\right.$ $\left.c_{q}\right)$ :

$$
\int_{\left[-\frac{1}{2}, \frac{1}{2}\right]^{2}} d p d q \operatorname{sgn}\left(c_{p}-c_{q}\right)\{\ldots\}=2 \int_{-1 / 2}^{1 / 2} d q \int_{-|q|}^{|q|} d p\{\ldots\}-\int_{\left[-\frac{1}{2}, \frac{1}{2}\right]^{2}} d p d q\{\ldots\} .
$$

For $\mathcal{L}_{m n}^{0}$, we treat the contribution from $\int_{\left[-\frac{1}{2}, \frac{1}{2}\right]^{2}} d p d q\{\ldots\}$ first. For $m=0$, one kind of Term survives: the terms in $\left(c_{n p}-c_{n q}\right) /\left(c_{p}-c_{q}\right)$, which depend on $q$ only: $2 c_{(n-1) q}, 2 c_{(n-3) q}$, and so on. This leads to

$$
\begin{gathered}
\frac{2}{\pi} \vartheta_{m} \vartheta_{n} \Delta_{m+n} \int_{\left[-\frac{1}{2}, \frac{1}{2}\right]^{2}} d p d q \frac{c_{n p}-c_{n q}}{c_{p}-c_{q}} c_{m p} f^{0}\left(\frac{1}{4}+q\right) f^{0}\left(\frac{1}{4}-q\right) \\
=\frac{2}{\pi} \vartheta_{m} \vartheta_{n} \Delta_{m+n} \int_{\left[-\frac{1}{2}, \frac{1}{2}\right]^{2}} d p d q\left\{\frac{c_{(n-2) p}-c_{(n-2) q}}{c_{p}-c_{q}}+2 c_{(n-1) p}\right. \\
+4 \sum_{l=1}^{n-2} \frac{c_{l p} c_{(n-1-l) q}}{\left(2 c_{(n-1) q}\right\}} c_{m p} f^{0}\left(\frac{1}{4}+q\right) f^{0}\left(\frac{1}{4}-q\right)
\end{gathered}
$$

We reduced the index $n$ in the factor $c_{n p}-c_{n q}$ by two and gained a term with 
$c_{(n-1) q}$. Iterating this procedure we end up with

$$
\begin{aligned}
& \frac{2}{\pi} \vartheta_{m} \vartheta_{n} \Delta_{m+n} \int_{\left[-\frac{1}{2}, \frac{1}{2}\right]^{2}} d p d q \frac{c_{n p}-c_{n q}}{c_{p}-c_{q}} c_{m p} f^{0}\left(\frac{1}{4}+q\right) f^{0}\left(\frac{1}{4}-q\right) \\
& =\frac{4}{\pi} \vartheta_{m} \vartheta_{n} \Delta_{m+n} \int_{-1 / 2}^{1 / 2} d q\left\{c_{(n-1) q}+c_{(n-3) q}+\ldots\right\} f^{0}\left(\frac{1}{4}+q\right) f^{0}\left(\frac{1}{4}-q\right) \int_{-1 / 2}^{1 / 2} d p c_{m p} .
\end{aligned}
$$

The integral over $p$ gives a factor $\delta_{m, 0}$, which makes the factor $\vartheta_{m}$ superfluous.

$$
\begin{aligned}
\frac{2}{\pi} & \vartheta_{m} \vartheta_{n} \Delta_{m+n} \int_{\left[-\frac{1}{2}, \frac{1}{2}\right]^{2}} d p d q \frac{c_{n p}-c_{n q}}{c_{p}-c_{q}} c_{m p} f^{0}\left(\frac{1}{4}+q\right) f^{0}\left(\frac{1}{4}-q\right) \\
& =\frac{4}{\pi} \delta_{m, 0} \vartheta_{n} \delta_{m, 0} \Delta_{n} \int_{-1 / 2}^{1 / 2} d q \sum_{j=1}^{n-1} \Delta_{j+1} c_{j q} f^{0}\left(\frac{1}{4}+q\right) f^{0}\left(\frac{1}{4}-q\right) \\
& =0
\end{aligned}
$$

The sum over $j$ starts at 1 , because of $\Delta_{n}$, which demands that $n$ is even. Therefore, the $c_{j p}$ are antisymmetric around $p=\frac{1}{4}$. With $f^{0}\left(\frac{1}{4} \pm q\right)$ being symmetric around $q=\frac{1}{4}$, this contribution to $\mathcal{L}_{m n}^{0}$ vanishes completely. Hence, we are left with the $\int_{-1 / 2}^{1 / 2} d q \int_{-|q|}^{|q|} d p\{\ldots\}$ part of $\mathcal{L}_{m n}^{0}$ :

$$
\begin{aligned}
\mathcal{L}_{m n}^{0}= & -\frac{4 U^{2}}{\pi \hbar J} \Delta_{m+n} \int_{-1 / 2}^{1 / 2} d q \int_{-|q|}^{|q|} d p\left\{\frac{c_{(n-2) p}-c_{(n-2) q}}{c_{p}-c_{q}}+2 c_{(n-1) p}\right. \\
& \left.+4 \sum_{l=1}^{n-2} c_{l p} c_{(n-1-l) q}+2 c_{(n-1) q}\right\} c_{m p} f^{0}\left(\frac{1}{4}+q\right) f^{0}\left(\frac{1}{4}-q\right) \\
=\vartheta_{n} & \hat{\mathcal{L}}_{m, n-2}-\frac{9 U^{2}}{\pi \hbar J} \vartheta_{m} \vartheta_{n} \Delta_{m+n} \int_{-1 / 2}^{1 / 2} d q\left\{\left[\frac{s_{(n+m-1)|q|}}{l+m}+\frac{s_{(n-m-1)|q|}}{l-m}\right]\right. \\
+ & \left.\frac{1}{\pi} \sum_{l=1}^{n-2}\left[\frac{s_{(l+m)|q|}}{l+m}+\frac{s_{(l-m)|q|}}{l-m}\right] c_{(n-1-l) q}+\delta_{m, 0} c_{(n-1) q}\right\} f^{0}\left(\frac{1}{4}+q\right) f^{0}\left(\frac{1}{4}-q\right) .
\end{aligned}
$$

Since $s_{(l+m)|q|}$ and $f^{0}\left(\frac{1}{4}+q\right) f^{0}\left(\frac{1}{4}-q\right)$ are symmetric around 0 and $\frac{1}{4}$, the integral over $q$ will only be non-zero if $c_{(n-1-l) q}$ is symmetric around 0 and $\frac{1}{4}$, too. Thus, some terms are dropping out:

$$
\begin{aligned}
\mathcal{L}_{m n}^{0}= & \frac{U^{2}}{\hbar J} \vartheta_{n} \mathcal{L}_{m, n-2}^{0}-\frac{32 U^{2}}{\pi \hbar J} \vartheta_{m} \vartheta_{n} \Delta_{m+n} \int_{0}^{1 / 4} d q\left\{\left[\frac{s_{(n+m-1) q}}{n+m-1}+\frac{s_{(n-m-1) q}}{n-m-1}\right]\right. \\
+ & \left.\frac{1}{\pi} \sum_{l=1}^{n-2}\left[\frac{s_{(n+m-l-1) q}}{n+m-l-1}+\frac{s_{(n-m-l-1) q}}{n-m-l-1}\right] \Delta_{l} c_{l q}\right\} f^{0}\left(\frac{1}{4}+q\right) f^{0}\left(\frac{1}{4}-q\right) .
\end{aligned}
$$

We got rid of the denominator $\left|c_{p}-c_{q}\right|$ and arrived at this form of $\mathcal{L}_{m n}^{0}$. 


\section{C.10 Degenerate non-hermitian perturbation the- ory}

In this section, we perform the perturbation theory on the operator $\hat{\mathcal{L}}^{\varepsilon=0}$ (see Sec. 3.6.4). For an introduction to perturbation theory in quantum mechanics, see for example Basdevant and Dalibard (2002).

The right kernel ker $\hat{\mathcal{L}}^{\varepsilon=0}$ consists of the constant function $\zeta_{0}$ and the functions $\zeta_{-|n|}$, which we call the low-lying eigenfunctions. We will find the corrections to their eigenvalues, which are called low-lying eigenvalues.

The other eigenfunctions of $\hat{\mathcal{L}}^{\varepsilon=0}$ we call the high-lying eigenfunctions. They span the remaining space $\left(\operatorname{ker} \hat{\mathcal{L}}^{\varepsilon=0}\right)^{\perp}$. We denote them as $\theta_{\mu}$.

$\hat{\mathcal{L}}$ is hermitian in the scalar product $\langle\cdot, \cdot\rangle_{\mathrm{F}}$, see Eqs. (3.14) and (B.64). Therefore, the left eigenfunctions are not the same as the right eigenfunctions. A left eigenfunction we denote by an upper index $L$. For every eigenfunction $\chi_{n}(k)$, we can find a left eigenfunction by multiplying with the factor $f(k)[1-f(k)]$, which is used in the scalar product:

$$
\chi_{n}^{L}(k)=\chi_{n}(k) f(k)[1-f(k)] .
$$

We introduce the Dirac notation

$$
\chi_{n}(k) \rightarrow\left|\chi_{n}\right\rangle, \quad \chi_{n}^{L}(k) \rightarrow\left\langle\chi_{n}\right| .
$$

Let $\psi^{\varepsilon}$ be a low-lying eigenfunction and $\lambda^{\varepsilon}$ its eigenvalue. Then, we may write

$$
\hat{\mathcal{L}}\left|\psi^{\varepsilon}\right\rangle=\lambda^{\varepsilon}\left|\psi^{\varepsilon}\right\rangle .
$$

Furthermore, we can expand $\left|\psi^{\varepsilon}\right\rangle$ using the eigenfunctions of $\hat{\mathcal{L}}$ :

$$
\left|\psi^{\varepsilon}\right\rangle=\sum_{n=-\infty}^{-1} A_{n}\left|v_{n}\right\rangle+\sum_{\mu} B_{\mu}\left|\theta_{\mu}\right\rangle .
$$

Since we only consider the low-lying eigenvalues, the zeroth order of the eigenvalue vanishes. Thus, one obtains

$$
\begin{array}{ll}
\hat{\mathcal{L}}^{\varepsilon=0}\left|v_{n \leqslant 0}\right\rangle=\lambda^{0}\left|v_{n \leqslant 0}\right\rangle=0 ; & \left\langle v_{n \leqslant-1}\right| \hat{\mathcal{L}}^{\varepsilon=0}=0, \\
\hat{\mathcal{L}}^{\varepsilon=0}\left|\theta_{\mu}\right\rangle=\nu_{\mu}\left|\theta_{\mu}\right\rangle ; & \left\langle\theta_{\mu}\right| \hat{\mathcal{L}}^{\varepsilon=0}=\nu_{\mu}\left\langle\theta_{\mu}\right| ; \quad \nu_{\mu} \in \mathbb{C} .
\end{array}
$$

Firstly, we apply $\left\langle v_{n}\right|$ onto Eq. (C.102) from the right:

$$
\begin{aligned}
& 0=\left\langle v_{n}\left|\hat{\mathcal{L}}-\lambda^{\varepsilon}\right| \psi^{\varepsilon}\right\rangle= \\
& =\underbrace{\left\langle v_{n}\right| \hat{\mathcal{L}}^{\varepsilon=0}-\lambda^{0}}_{=0}\left|\psi^{\varepsilon}\right\rangle+\varepsilon\left\langle v_{n}\left|\hat{\mathcal{L}}_{1}-\tilde{\lambda}_{1}\right| \psi^{\varepsilon}\right\rangle+\varepsilon^{2}\left\langle v_{n}\left|\hat{\mathcal{L}}_{2}-\tilde{\lambda}_{2}\right| \psi^{\varepsilon}\right\rangle+\mathcal{O}\left(\varepsilon^{3}\right) \\
& =\varepsilon \sum_{m} A_{m}\left\langle v_{n}\left|\hat{\mathcal{L}}_{1}\right| v_{m}\right\rangle+\varepsilon \sum_{\mu} B_{\mu}\left\langle v_{n}\left|\hat{\mathcal{L}}_{1}\right| \theta_{\mu}\right\rangle-\varepsilon \tilde{\lambda}_{1} A_{n}+\mathcal{O}\left(\varepsilon^{2}\right)
\end{aligned}
$$


This leads to

$$
\tilde{\lambda}_{1} A_{n}=\sum_{m} A_{m}\left\langle v_{n}\left|\hat{\mathcal{L}}_{1}\right| v_{m}\right\rangle+\sum_{\mu} B_{\mu}\left\langle v_{n}\left|\hat{\mathcal{L}}_{1}\right| \theta_{\mu}\right\rangle+\mathcal{O}(\varepsilon) .
$$

Secondly, we do the same with $\left\langle\theta_{\mu}\right|$ :

$$
\begin{aligned}
0 & =\left\langle\theta_{\mu}\left|\hat{\mathcal{L}}-\lambda^{\varepsilon}\right| \psi^{\varepsilon}\right\rangle \\
& =\left\langle\theta_{\mu}\left|\hat{\mathcal{L}}^{\varepsilon=0}-\lambda^{0}\right| \psi^{\varepsilon}\right\rangle+\varepsilon\left\langle\theta_{\mu}\left|\hat{\mathcal{L}}_{1}-\tilde{\lambda}_{1}\right| \psi^{\varepsilon}\right\rangle+\varepsilon^{2}\left\langle\theta_{\mu}\left|\hat{\mathcal{L}}_{2}-\tilde{\lambda}_{2}\right| \psi^{\varepsilon}\right\rangle+\mathcal{O}\left(\varepsilon^{3}\right) \\
& =\nu_{\mu} B_{\mu}+\varepsilon \sum_{n} A_{n}\left\langle\theta_{\mu}\left|\hat{\mathcal{L}}_{1}\right| v_{n}\right\rangle+\varepsilon \sum_{\mu} B_{\mu}\left\langle\theta_{\mu}\left|\hat{\mathcal{L}}_{1}\right| \theta_{\mu}\right\rangle+\mathcal{O}\left(\varepsilon^{2}\right)
\end{aligned}
$$

For $\nu_{\mu} \neq 0$, we deduce that

$$
B_{\mu}=\mathcal{O}(\varepsilon)
$$

Plugging this into Eq. (C.105) we find.

$$
\tilde{\lambda}_{1} A_{n}=\sum_{m} A_{m}\left\langle v_{n}\left|\hat{\mathcal{L}}_{1}\right| v_{m}\right\rangle+\mathcal{O}(\varepsilon) .
$$

Therefore, the first-order contribution $\tilde{\lambda}_{1}$ to $\lambda^{\varepsilon}$ is defined by the eigenvalue problem

$$
\sum_{n=-\infty}^{-1} \mathcal{L}_{m n}^{(1)} A_{n}=\tilde{\lambda}_{1} A_{m}
$$

with

$$
\mathcal{L}_{m n}^{(1)}=\left\langle v_{n}\left|\hat{\mathcal{L}}_{1}\right| v_{m}\right\rangle=\left\langle\zeta_{n}^{L}, \hat{\mathcal{L}}_{1}\left[\zeta_{m}\right]\right\rangle=\left\langle\zeta_{n}, \hat{\mathcal{L}}_{1}\left[\zeta_{m}\right]\right\rangle_{\mathrm{F}}
$$

This result gives Eq. (3.165).

\section{C.11 Computing the first order correction of $\hat{\mathcal{L}}$}

Here, we execute the first-order perturbation theory calculation from Sec. 3.6.4.

$$
\begin{aligned}
\mathcal{L}_{m n}^{(1)} \equiv\left\langle v_{m}, \hat{\mathcal{L}}_{1}\left[v_{n}\right]\right\rangle_{\mathrm{F}} \equiv\left\langle v_{m}\left|\left[\frac{d}{d \varepsilon} \hat{\mathcal{L}}\right]_{\varepsilon=0}\right| v_{n}\right\rangle=\left[\frac{d}{d \varepsilon} \mathcal{L}_{m n}^{\varepsilon}\right]_{\varepsilon=0} \\
\stackrel{(3.157)}{=}-\frac{U^{2}}{\hbar J} \Delta_{m+n} \int d p d q \frac{\gamma(0)}{\left|c_{p}-c_{q}\right|}\left\{\frac{d}{d \varepsilon} \zeta_{m}\left(\tilde{k}_{p q}^{\varepsilon}+p\right)\right. \\
\times\left[1-f\left(\tilde{k}_{p q}^{\varepsilon}+p\right)\right]\left[1-f\left(\tilde{k}_{p q}^{\varepsilon}-p\right)\right] f\left(\tilde{k}_{p q}^{\varepsilon}+q\right) f\left(\tilde{k}_{p q}^{\varepsilon}-q\right) \\
\left.\times\left[v_{n}\left(\tilde{k}_{p q}^{\varepsilon}+p\right)+v_{n}\left(\tilde{k}_{p q}^{\varepsilon}-p\right)-v_{n}\left(\tilde{k}_{p q}^{\varepsilon}+q\right)-v_{n}\left(\tilde{k}_{p q}^{\varepsilon}-q\right)\right]\right\}_{\varepsilon=0} .
\end{aligned}
$$

This equation is Eq. (3.167) with the difference that we already pulled $\gamma(0)$ out of the derivative. We can do that, because $\gamma$ is an even function and therefore $\frac{d}{d \varepsilon} \gamma\left(\varepsilon r_{p q}\right)=0$. 
First, we expand all other functions up to first order in $\varepsilon$. For this, we make use of $\tilde{k}_{p q}^{\varepsilon}=\frac{1}{4}-\frac{1}{\pi} \varepsilon r_{p q}+\mathcal{O}\left(\varepsilon^{3}\right)$, given in Eq. (3.44). For $\zeta_{n}$, defined in Eq. (3.149), we find

$$
v_{n}\left(\tilde{k}_{p q}^{\varepsilon}+p\right)=\bar{\vartheta}_{n} s_{|n| p}+\vartheta_{n} c_{n p}+\varepsilon n r_{p q}\left[\bar{\vartheta}_{n} c_{|n| p}+\vartheta_{n} s_{n p}\right]+\mathcal{O}\left(\varepsilon^{2}\right) .
$$

For the other factors depending on $\varepsilon$, one obtains

$$
f\left(\tilde{k}_{p q}^{\varepsilon}+p\right)=f^{0}\left(\frac{1}{4}+p\right)+\varepsilon f^{0}\left(\frac{1}{4}+p\right)\left[1-f^{0}\left(\frac{1}{4}+p\right)\right]\left[\frac{d}{d \varepsilon} \beta \omega\left(\tilde{k}_{p q}^{\varepsilon}+p\right)\right]_{\varepsilon=0}+\mathcal{O}\left(\varepsilon^{2}\right) .
$$

Thus, we need to calculate

$$
\begin{aligned}
& {\left[\frac{d}{d \varepsilon} \omega\left(\tilde{k}_{p q}^{\varepsilon}+p\right)\right]_{\varepsilon=0}=\left[\frac{\partial \omega}{\partial \varepsilon}\left(\tilde{k}_{p q}^{\varepsilon}+p\right)+\left.\frac{\partial \omega(\kappa)}{\partial \kappa}\right|_{\kappa=\tilde{k}_{p q}^{\varepsilon}+p} \frac{\partial \tilde{k}_{p q}^{\varepsilon}}{\partial \varepsilon}\right]_{\varepsilon=0}} \\
& =\left[-\tilde{c}_{\tilde{k}_{p q}^{\varepsilon}+p}+2 \pi\left[s_{\tilde{k}_{p q}^{\varepsilon}+p}+2 \varepsilon \tilde{s}_{\tilde{k}_{p q}^{\varepsilon}=p}\right]\left(-\frac{r_{p q}}{\pi}+\mathcal{O}\left(\varepsilon^{2}\right)\right)\right]_{\varepsilon=0} \\
& =\tilde{c}_{p}-2 c_{p} r_{p q} \\
& =-1-2 c_{p} c_{q} .
\end{aligned}
$$

Note that partial derivative with respect to $\varepsilon$ means that only the explicit occurrence of $\varepsilon$ in $\omega(k)$ is taken into account, i.e. $\partial_{\varepsilon} \omega(k)=\partial_{\varepsilon}[-\sin (2 \pi k)-$ $\varepsilon \cos (2 \pi k)]=-\cos (2 \pi k)$. Now, we can utilize C.115 to obtain

$$
f\left(\tilde{k}_{p q}^{\varepsilon}+p\right)=f^{0}\left(\frac{1}{4}+p\right)\left\{1-\beta \varepsilon\left[1-f^{0}\left(\frac{1}{4}+p\right)\right]\left(1+2 c_{p} c_{q}\right)\right\}+\mathcal{O}\left(\varepsilon^{2}\right) .
$$

In order to express the Fermi factor in Eq. (C.112), we need the following product of two Fermi functions:

$$
\begin{aligned}
f\left(\tilde{k}_{p q}^{\varepsilon}+p\right) f\left(\tilde{k}_{p q}^{\varepsilon}-p\right)= & f^{0}\left(\frac{1}{4}+p\right) f^{0}\left(\frac{1}{4}-p\right)\left[1+\beta \varepsilon\left(1+2 c_{p} c_{q}\right)\right. \\
& \left.\times\left[1-f^{0}\left(\frac{1}{4}+p\right)+1-f^{0}\left(\frac{1}{4}-p\right)\right]\right]+\mathcal{O}\left(\varepsilon^{2}\right) .
\end{aligned}
$$

Furthermore, also the calculation of the sum of these Fermi functions is necessary:

$$
\begin{aligned}
f^{0}\left(\frac{1}{4}+p\right)+f^{0}\left(\frac{1}{4}-p\right) & =\frac{1}{1+\exp \left(-\beta c_{\frac{1}{4}+p}-\beta \mu\right)}+\frac{1}{1+\exp \left(-\beta c_{\frac{1}{4}-p}-\beta \mu\right)} \\
& =\frac{1}{1+\exp \left(\beta s_{p}-\beta \mu\right)}+\frac{1}{1+\exp \left(-\beta s_{p}-\beta \mu\right)} \\
& =\frac{2+\exp \left(\beta s_{p}-\beta \mu\right)+\exp \left(-\beta s_{p}-\beta \mu\right)}{1+\exp \left(\beta s_{p}-\beta \mu\right)+\exp \left(-\beta s_{p}-\beta \mu\right)+\exp (-2 \beta \mu)} \\
& =\frac{2+2 e^{-\beta \mu} \cosh \left(\beta s_{p}\right)}{1+e^{-2 \beta \mu}+2 e^{-\beta \mu} \cosh \left(\beta s_{p}\right)} .
\end{aligned}
$$

This function is equal to one for $\mu=0$. To shorten notation, we define the following function, which is one for $\mu=0$ and even in $p$ :

$$
\eta(p, \beta, \beta \mu):=2-f^{0}\left(\frac{1}{4}+p\right)-f^{0}\left(\frac{1}{4}-p\right)=\frac{2 e^{-2 \beta \mu}+2 e^{-\beta \mu} \cosh \left(\beta s_{p}\right)}{1+e^{-2 \beta \mu}+2 e^{-\beta \mu} \cosh \left(\beta s_{p}\right)} .
$$


For the sake of an even shorter notation, we will not explicitly write the parameters $\beta$ and $\beta \mu$. This leads to

$$
f\left(\tilde{k}_{p q}^{\varepsilon}+p\right) f\left(\tilde{k}_{p q}^{\varepsilon}-p\right)=f^{0}\left(\frac{1}{4}+p\right) f^{0}\left(\frac{1}{4}-p\right)\left[1+\beta \varepsilon \eta(p)\left(1+2 c_{p} c_{q}\right)\right]+\mathcal{O}\left(\varepsilon^{2}\right) .
$$

All the factors like Eqs. (C.113) and (C.120) have a linear term in $\varepsilon$. Therefore, they contribute to $\mathcal{L}_{m n}^{(1)}$. Plugging them into Eq. (C.112) yields

$$
\begin{aligned}
\mathcal{L}_{m n}^{(1)}= & -\frac{U^{2} \Delta_{m+n}}{\hbar J} \int_{\left[-\frac{1}{2}, \frac{1}{2}\right]^{2}} d p d q \frac{\gamma(0)}{\left|c_{p}-c_{q}\right|} f_{p+}^{0} f_{p-}^{0} f_{q+}^{0} f_{q-}^{0}\left\{\left[m r_{p q}\left[\bar{\vartheta}_{m} c_{|m| p}+\vartheta_{m} s_{m p}\right]\right.\right. \\
& \left.-\beta\left(\bar{\vartheta}_{m} s_{|m| p}+\vartheta_{m} c_{m p}\right)\left(1+2 c_{p} c_{q}\right)[\eta(p)+\eta(q)]\right] \cdot\left(2 \vartheta_{n} c_{n p}-2 \vartheta_{n} c_{n q}\right) \\
+ & \left.\left(\bar{\vartheta}_{m} s_{|m| p}+\vartheta_{m} c_{m p}\right) \cdot\left(2 n r_{p q} \bar{\vartheta}_{n} c_{n p}-2 n r_{p q} \bar{\vartheta}_{n} c_{n q}\right)\right\}
\end{aligned}
$$

We use $\gamma(0)=1$, see Eq. (3.73), and make some simplifications so that we arrive at

$$
\begin{aligned}
\mathcal{L}_{m n}^{(1)}=- & \frac{2 U^{2} \Delta_{m+n}}{\hbar J} \int_{\left[-\frac{1}{2}, \frac{1}{2}\right]^{2}} d p d q \frac{c_{n p}-c_{n q}}{\left|c_{p}-c_{q}\right|} f_{p+}^{0} f_{p-}^{0} f_{q+}^{0} f_{q-}^{0} \\
& \times\left\{\vartheta _ { n } \left[m r_{p q}\left[\bar{\vartheta}_{m} c_{|m| p}+\vartheta_{m} s_{m p}\right]\right.\right. \\
& \left.-\beta\left(\bar{\vartheta}_{m} s_{|m| p}+\vartheta_{m} c_{m p}\right)\left(1+2 c_{p} c_{q}\right)[\eta(p)+\eta(q)]\right] \\
+ & \left.\bar{\vartheta}_{n} n r_{p q}\left(\bar{\vartheta}_{m} s_{|m| p}+\vartheta_{m} c_{m p}\right)\right\} .
\end{aligned}
$$

The parts of the integrand, which are odd in $p$, drop out. These are all the terms containing a sinus factor:

$$
\begin{aligned}
\mathcal{L}_{m n}^{(1)}=- & \frac{2 U^{2} \Delta_{m+n}}{\hbar J} \int d p d q c_{m p} \frac{c_{n p}-c_{n q}}{\left|c_{p}-c_{q}\right|} f_{\left.p+\frac{1}{2}, \frac{1}{2}\right]^{2}}^{0} f_{p-}^{0} f_{q+}^{0} f_{q-}^{0}\left\{\vartheta _ { n } \left[m r_{p q} \bar{\vartheta}_{m}\right.\right. \\
& \left.\left.-\beta \vartheta_{m}\left(1+2 c_{p} c_{q}\right)[\eta(p)+\eta(q)]\right]+\bar{\vartheta}_{n} n r_{p q} \vartheta_{m}\right\}
\end{aligned}
$$

Now, we consider the transformation $(p, q) \rightarrow\left(\frac{1}{2}-p, \frac{1}{2}-q\right)$. In this transformation, $r_{p q}=c_{p}+c_{q}$ gets a minus sign. The function $\eta(p)$ does not change, because $\cosh \left(\beta s_{p}\right)$ is invariant under this transformation. Furthermore, the factor $\Delta_{m+n} c_{m p}\left(c_{n p}-c_{n q}\right) /\left|c_{p}-c_{q}\right|$ is invariant under this transformation. Consequently, the two terms with $r_{p q}$ vanish:

$$
\begin{aligned}
\mathcal{L}_{m n}^{(1)}= & \frac{2 U^{2} \beta}{\hbar J} \vartheta_{m} \vartheta_{n} \Delta_{m+n} \int d p d q c_{m p} \frac{c_{n p}-c_{n q}}{\left|c_{p}-c_{q}\right|}\left(1+2 c_{p} c_{q}\right) \\
& \times f^{0}\left(\frac{1}{4}+p\right) f^{0}\left(\frac{1}{4}-p\right) f^{0}\left(\frac{1}{4}+q\right) f^{0}\left(\frac{1}{4}-q\right)[\eta(p)+\eta(q)] .
\end{aligned}
$$


We can anti-symmetrize the factor $c_{m p}$, since the rest is antisymmetric under exchange of $p$ and $q$ :

$$
\begin{aligned}
\mathcal{L}_{m n}^{(1)}= & \frac{U^{2} \beta}{\hbar J} \vartheta_{m} \vartheta_{n} \Delta_{m+n} \int_{\left[-\frac{1}{2}, \frac{1}{2}\right]^{2}} d p d q \frac{1+2 c_{p} c_{q}}{\left|c_{p}-c_{q}\right|}\left(c_{m p}-c_{m q}\right)\left(c_{n p}-c_{n q}\right) \\
& \times f^{0}\left(\frac{1}{4}+p\right) f^{0}\left(\frac{1}{4}-p\right) f^{0}\left(\frac{1}{4}+q\right) f^{0}\left(\frac{1}{4}-q\right)[\eta(p)+\eta(q)] .
\end{aligned}
$$

The factor $\vartheta_{n}\left(c_{n p}-c_{n q}\right)$ tells us that for $n \leqslant 0$ the matrix elements $\mathcal{L}_{m n}^{(1)}$ vanish. The same holds for $m \leqslant 0$. This leads to Eq. (3.169) in the main text.

\section{C.12 The relation between Fermi momentum and chemical potential}

The relation between Fermi momentum $k_{\mathrm{F}}$ and chemical potential $\mu$ is defined by

$$
\mu=\omega\left(k_{\mathrm{F}}\right)=-c_{F}-\varepsilon\left(2 c_{F}^{2}-1\right),
$$

with $c_{F}:=\cos \left(2 \pi k_{\mathrm{F}}\right)$. This is how one calculates $\mu$ from $k_{\mathrm{F}}$. However, we want to know the inverse function. Therefore, we reorder Eq. (C.126) to

$$
2 \varepsilon c_{F}^{2}+c_{F}-\varepsilon+\mu=0 .
$$

We use the known solution of quadratic equations and get

$$
c_{F}=\frac{-1 \pm \sqrt{1+8 \varepsilon(\varepsilon-\mu)}}{4 \varepsilon} .
$$

For $\varepsilon<\frac{1}{4}$, the solution is the one with the plus sign. The other solution would lead to the right-hand side exceeding the range of the cosine. Thus, we have

$$
k_{\mathrm{F}}=\frac{1}{2 \pi} \arccos \left[\frac{\sqrt{1+8 \varepsilon(\varepsilon-\mu)}-1}{4 \varepsilon}\right] \text {. }
$$

The Taylor expansion in $\varepsilon$ yields

$$
k_{\mathrm{F}}=\frac{1}{2}-\frac{\arccos (\mu)}{2 \pi}-\frac{1-2 \mu^{2}}{2 \pi \sqrt{1-\mu^{2}}} \varepsilon .
$$

For the case $\mu=0$, which is very often considered in this work, we get

$$
\left.k_{\mathrm{F}}\right|_{\mu=0}=\frac{1}{2 \pi} \arccos \left[\frac{\sqrt{1+8 \varepsilon^{2}}-1}{4 \varepsilon}\right]
$$

and the corresponding Taylor expansion

$$
\left.k_{\mathrm{F}}\right|_{\mu=0}=\frac{1}{4}-\frac{\varepsilon}{2 \pi}+\frac{11 \varepsilon^{3}}{12 \pi}+\mathcal{O}\left(\varepsilon^{5}\right) .
$$

We see that for $\mu=0$ the Fermi momentum $k_{F}$ is shifted below $\frac{1}{4}$ by $\mathcal{O}(\varepsilon)$. 


\section{C.13 The factor containing the Fermi-Dirac func- tions}

In this section, we will be concerned with a certain factor in the definition of the linearized Boltzmann operator $\hat{\mathcal{L}}$, Eq. (3.12):

$$
F_{1234}:=\frac{1-f_{2}}{f_{1}} f_{3} f_{4} .
$$

We will always keep in mind that we have the momentum conservation $\operatorname{kmod}\left(k_{1}+\right.$ $\left.k_{2}-k_{3}-k_{4}\right)=0$ and the energy conservation $\omega_{1}+\omega_{2}-\omega_{3}-\omega_{4}=0$. The latter leads to

$$
\left(1-f_{1}\right)\left(1-f_{2}\right) f_{3} f_{4}=f_{1} f_{2}\left(1-f_{3}\right)\left(1-f_{4}\right),
$$

see Eq. (B.59). Thus, we can rewrite $F_{1234}$ by multiplying it with $1=1-f_{1}+f_{1}$ :

$$
\begin{aligned}
F_{1234} & =\frac{\left(1-f_{1}+f_{1}\right)\left(1-f_{2}\right) f_{3} f_{4}}{f_{1}} \\
& =\frac{\left(1-f_{1}\right)\left(1-f_{2}\right) f_{3} f_{4}}{f_{1}}+\frac{f_{1}\left(1-f_{2}\right) f_{3} f_{4}}{f_{1}} .
\end{aligned}
$$

For the first term, we use Eq. (C.134). In the second term, we simply cancel $f_{1}$ :

$$
\begin{aligned}
F_{1234} & =\frac{f_{1} f_{2}\left(1-f_{3}\right)\left(1-f_{4}\right)}{f_{1}}+\left(1-f_{2}\right) f_{3} f_{4} \\
& =f_{2}\left(1-f_{3}\right)\left(1-f_{4}\right)+\left(1-f_{2}\right) f_{3} f_{4} .
\end{aligned}
$$

With this we found a way to write $F_{1234}$ in such a way that there is no denominator any more. Although $f_{1}$ vanished, $F_{1234}$ is still dependent on $k_{1}$ because of quasi-momentum-conservation and energy conservation. The $T \rightarrow 0$ limit is now very easily found:

$$
F_{1234} \stackrel{T \rightarrow 0}{\longrightarrow} \theta_{2} \bar{\theta}_{3} \bar{\theta}_{4}+\bar{\theta}_{2} \theta_{3} \theta_{4},
$$

where we introduced $\theta_{j}:=\theta\left(-\omega_{j}+\mu\right)$ and $\bar{\theta}_{j}=\theta\left(\omega_{j}-\mu\right)$.

One can also multiply Eq. (C.133) by $1=\theta_{1}+\bar{\theta}_{1}$. This leads to

$$
F_{1234}=\frac{\theta_{1}\left(1-f_{2}\right) f_{3} f_{4}}{f_{1}}+\frac{\bar{\theta}_{1}\left(1-f_{2}\right) f_{3} f_{4}}{f_{1}} .
$$

The first term is not a problem in the limit $T \rightarrow 0$, because as long as $\omega_{1}<\mu$ the Fermi function approaches 1 . However, in the second term, the fact that $\omega_{1}>\mu$ lets the denominator consisting of $f_{1}$ vanish. We rewrite the second term using Eq. Eq. (C.134):

$$
F_{1234}=\frac{\theta_{1}\left(1-f_{2}\right) f_{3} f_{4}}{f_{1}}+\frac{\bar{\theta}_{1} f_{2}\left(1-f_{3}\right)\left(1-f_{4}\right)}{1-f_{1}} .
$$

The $T \rightarrow 0$ limit leads to

$$
F_{1234} \stackrel{T \rightarrow 0}{\longrightarrow} \theta_{1} \bar{\theta}_{2} \theta_{3} \theta_{4}+\bar{\theta}_{1} \theta_{2} \bar{\theta}_{3} \bar{\theta}_{4} .
$$


This seems to be contradictory to Eq. (C.137). The additional factors $\theta_{1}$ and $\bar{\theta}_{1}$ are redundant. For example, the term $\bar{\theta}_{2} \theta_{3} \theta_{4}$ leads to

$$
\omega_{1}=\omega_{3}+\omega_{4}-\omega_{2}<\mu+\mu-\mu=\mu .
$$

This is equivalent to a factor $\theta\left(-\omega_{1}+\mu\right)=\bar{\theta}_{1}$. Hence, both Eqs. (C.137) and (C.140) are valid and equivalent. Summing up the important things leads to

$$
F_{1234}=f_{2}\left(1-f_{3}\right)\left(1-f_{4}\right)+\left(1-f_{2}\right) f_{3} f_{4} \text {, }
$$

and

$$
\begin{aligned}
F_{1234} \stackrel{T \rightarrow 0}{\longrightarrow} \bar{\theta}_{2} \theta_{3} \theta_{4}+\theta_{2} \bar{\theta}_{3} \bar{\theta}_{4} \\
\quad=\theta_{1} \bar{\theta}_{2} \theta_{3} \theta_{4}+\bar{\theta}_{1} \theta_{2} \bar{\theta}_{3} \bar{\theta}_{4} .
\end{aligned}
$$




\section{Appendix D}

\section{Calculations for the PCMO chain}

\section{D.1 Evaluation of the energy-conserving delta- function using paths}

In this section, we show how one can reformulate a delta function of the form $\delta(h(x, y))$ using paths. This is used in the numerical evaluation of the energyconserving delta-function $\Delta \omega_{\vec{\nu}, \vec{k}}$, see Sec. 4.3.2. Since $k_{1}$ is a parameter and $k_{4}$ is set by the momentum conservation $\delta\left(\Delta k_{\vec{k}}\right)$, two parameters are left to integrate over, i.e. $(x, y)=\left(k_{2}, k_{3}\right)$. The problem can be formulated as

$$
\int d x d y \delta(h(x, y)) f(x, y) .
$$

For the beginning, we assume that the function $h(x, y)$ contains one path $\gamma$, where its function value vanishes. Hence, we have

$$
h(\gamma(t))=0 .
$$

This is valid for all path parameters $t$. Assuming that the paths are smooth, we can take the derivative of this equation and find

$$
0=\frac{d}{d t} h(\gamma(t))=\gamma^{\prime}(t) \cdot \nabla_{\gamma} h(\gamma(t))
$$

We now see that $\nabla h(\gamma(t))$ is orthogonal to $\gamma^{\prime}(t)$. Therefore, we can now specify the following reparametrization of the integral near the path $\gamma$ :

$$
(x, y)=\gamma(t)+s \nabla_{\gamma} h(\gamma(t)) .
$$

Thus, Eq. (D.1) takes the form

$$
\begin{aligned}
& \int d x d y \delta(h(x, y)) f(x, y) \\
& =\int d t d s\left|\frac{\partial(x, y)}{\partial(t, s)}\right| \delta\left(h\left(\gamma(t)+s \nabla_{\gamma} h(\gamma(t))\right)\right) f\left(\gamma(t)+s \nabla_{\gamma} h(\gamma(t))\right)
\end{aligned}
$$


The reparametrization includes the Jacobi determinant

$$
\begin{aligned}
\left|\frac{\partial(x, y)}{\partial(t, s)}\right| & =\left|\frac{\partial x}{\partial t} \frac{\partial y}{\partial s}-\frac{\partial x}{\partial s} \frac{\partial y}{\partial t}\right| \\
& =\left|\gamma_{1}^{\prime}(t) \frac{\partial h(\gamma(t))}{\partial y}-\gamma_{2}^{\prime}(t) \frac{\partial h(\gamma(t))}{\partial x}\right| \\
& =\left\|\gamma^{\prime}(t)\right\|\left\|\nabla_{\gamma} h(\gamma(t))\right\| .
\end{aligned}
$$

In the last step, we made use of the orthogonality of $\gamma^{\prime}(t)$ and $\nabla_{\gamma} h(\gamma(t))$, because for two orthogonal functions $v$ and $w$ we have

$$
\begin{aligned}
\|v\|^{2}\|w\|^{2} & =\left(v_{1}^{2}+v_{2}^{2}\right)\left(w_{1}^{2}+w_{2}^{2}\right)=\underbrace{\left(v_{1} w_{1}+v_{2} w_{2}\right)^{2}}_{=0}-2 v_{1} v_{2} w_{1} w_{2}+v_{1}^{2} w_{2}^{2}+v_{2} w_{1}^{2} \\
& =\left(v_{1} w_{2}-v_{2} w_{1}\right)^{2} .
\end{aligned}
$$

Furthermore, for the delta function, we consider the Taylor expansion

$$
\begin{aligned}
& h\left(\gamma(t)+s \nabla_{\gamma} h(\gamma(t))\right) \\
& =\sum_{m, n=0}^{\infty} \frac{s^{m+n}}{m ! n !} \frac{\partial^{m} \partial^{n} h(\gamma(t))}{\partial x^{m} \partial y^{n}}\left[\frac{\partial h(\gamma(t))}{\partial x}\right]^{m}\left[\frac{\partial h(\gamma(t))}{\partial y}\right]^{n} \\
& =\underbrace{h(\gamma(t))}_{=0}+s \nabla_{\gamma} h(\gamma(t)) \cdot \nabla_{\gamma} h(\gamma(t)) \\
& +s^{2} \underbrace{\sum_{\substack{m, n=0 \\
m+1 \geqslant 2}}^{\infty} \frac{s^{m+n-2}}{m ! n !} \frac{\partial^{m} \partial^{n} h(\gamma(t))}{\partial x^{m} \partial y^{n}}\left[\frac{\partial h(\gamma(t))}{\partial x}\right]^{m}\left[\frac{\partial h(\gamma(t))}{\partial y}\right]^{n}}_{=: Q} .
\end{aligned}
$$

This leads to

$$
\begin{aligned}
\delta\left(h\left(\gamma(t)+s \nabla_{\gamma} h(\gamma(t))\right)\right) & =\delta\left(s\left\|\nabla_{\gamma} h(\gamma(t))\right\|^{2}+s^{2} Q\right) \\
& =\frac{\delta(s)}{\left\|\nabla_{\gamma} h(\gamma(t))\right\|^{2}}+\delta\left(\left\|\nabla_{\gamma} h(\gamma(t))\right\|^{2}+s Q\right) .
\end{aligned}
$$

In our case, the second term does not contribute, because we assumed that we have only one path. If there is another path crossing or touching the path, the contributions are taken into account by simply summing over all possible paths.

We obtain for Eq. (D.5) the following result:

$$
\begin{aligned}
& \int d x d y \delta(h(x, y)) f(x, y) \\
& =\int d t d s\left\|\gamma^{\prime}(t)\right\|\left\|\nabla_{\gamma} h(\gamma(t))\right\| \frac{\delta(s)}{\left\|\nabla_{\gamma} h(\gamma(t))\right\|^{2}} f\left(\gamma(t)+s \nabla_{\gamma} h(\gamma(t))\right) \\
& =\int d t \frac{\left\|\gamma^{\prime}(t)\right\|}{\left\|\nabla_{\gamma} h(\gamma(t))\right\|} f(\gamma(t)) .
\end{aligned}
$$

If $h(x, y)$ contains more than one path with $h(\gamma(t))=0$, then we have to sum over all paths. This leads to the final result:

$$
\int d x d y \delta(h(x, y)) f(x, y)=\sum_{\gamma} \int d t \frac{\left\|\gamma^{\prime}(t)\right\|}{\left\|\nabla_{\gamma} h(\gamma(t))\right\|} f(\gamma(t))
$$




\section{D.2 First derivative of the paths of zero energy change}

Here, we calculate the first derivative $\gamma^{\prime}(x)$ of the paths introduced in App. D.1. This is used in Sec. 4.3.2. $\gamma^{\prime}(x)$ can be calculated in a similar manner as the interpolation scheme discussed in App. B.5. However, here we do not need to interpolate between points, we only want to find the derivative at the discretized points $x_{l}$, which simplifies the calculation.

$$
\begin{aligned}
\gamma\left(x_{l}\right) & =\sum_{n=0}^{\infty} \frac{1}{n !} \gamma^{(n)}\left(x_{m}\right)\left(x_{l}-x_{m}\right)^{n} \\
& =\sum_{n=0}^{\infty} \frac{1}{n !} \gamma^{(n)}\left(x_{m}\right)(l-m)^{n} \Delta x^{n}
\end{aligned}
$$

We choose the parametrization so that $x_{l}=l \Delta x, x \in\left[0, N_{t}\right)$. Furthermore, we need to specify which, points we use for approximating $\gamma^{\prime}\left(x_{l}\right)$. The number of the points is $N_{\mathrm{I}}$. We use the indices in $\{l+a, \ldots, l+b\}$ with $b=$ floor $\left(N_{\mathrm{I}} / 2\right)$ and $b-a=N_{\mathrm{I}}-1$.

$$
\begin{aligned}
& \gamma^{\prime}\left(x_{l}\right)=\sum_{m=l+a}^{l+b} \tilde{C}_{m}\left(x_{l}\right) \gamma\left(x_{m}\right)=\sum_{n=0}^{\infty} \gamma^{(n)}\left(x_{l}\right) \frac{\Delta x^{n}}{n !} \sum_{m=l+a}^{l+b} \tilde{C}_{m}\left(x_{l}\right)(m-l)^{n} \\
& =\gamma\left(x_{l}\right) \underbrace{\sum_{m=a}^{b} \tilde{C}_{m}\left(x_{l}\right)}_{\stackrel{!}{=} 0}+\gamma^{\prime}\left(x_{l}\right) \underbrace{\Delta x \sum_{m=a}^{b} \tilde{C}_{l+m}\left(x_{l}\right) m}_{\stackrel{!}{=} 1}+\sum_{n=2}^{\infty} \gamma^{(n)}\left(x_{l}\right) \underbrace{\frac{\Delta x^{n}}{n !} \sum_{m=a}^{b} \tilde{C}_{l+m}\left(x_{l}\right) m^{n}}_{\stackrel{!}{=} 0} .
\end{aligned}
$$

Let us define

$$
C_{m}:=\Delta x \tilde{C}_{l+m}\left(x_{l}\right)
$$

It fulfills

$$
\left[\begin{array}{cccc}
1 & 1 & \ldots & 1 \\
a & a+1 & \ldots & b \\
\vdots & \vdots & & \vdots \\
a^{N_{\mathrm{I}}-1} & (a+1)^{N_{\mathrm{I}}-1} & \ldots & b^{N_{\mathrm{I}}-1}
\end{array}\right] \vec{C}=\left[\begin{array}{c}
0 \\
1 \\
0 \\
\vdots \\
0
\end{array}\right]
$$

We can see that $C_{m}(l)$ is only dependent on the number of points $N_{\mathrm{I}}$. This is very useful for the numerical implementation of the linearized Boltzmann equation, because we only need to calculate $C_{m}$ once and can use it for any choice of $\Delta x$ and number of path points $x_{l}$ of the path $\gamma(x)$.

We can rewrite Eq. (D.15) by defining $\vec{y}:=(a, a+1, \ldots, b)$ :

$$
\left[\begin{array}{cccc}
1 & 1 & \ldots & 1 \\
y_{1} & y_{2} & \ldots & y_{N_{\mathrm{I}}} \\
\vdots & \vdots & & \vdots \\
y_{1}^{N_{\mathrm{I}}-1} & y_{2}^{N_{\mathrm{I}}-1} & \ldots & y_{N_{\mathrm{I}}}^{N_{\mathrm{I}}-1}
\end{array}\right] \vec{C}(k)=\left[\begin{array}{c}
0 \\
1 \\
0 \\
\vdots \\
0
\end{array}\right]
$$


We solved this using Mathematica and found the following principle, which we checked up to the desired $N_{\mathrm{I}}=9$ :

$$
C_{n}=(-1)^{N_{\mathrm{I}}} \frac{\sum_{\substack{l=1 \\ l \neq n}}^{N_{\mathrm{I}}} \prod_{\substack{m=1 \\ m \neq n \\ m \neq l}}^{N_{\mathrm{I}}} y_{m}}{\prod_{\substack{m=1 \\ m \neq n}}^{N_{\mathrm{I}}}\left(y_{n}-y_{m}\right)}=(-1)^{N_{\mathrm{I}}} \frac{\sum_{\substack{l=1 \\ l \neq n}}^{N_{\mathrm{I}}} \prod_{\substack{m=1 \\ m \neq n \\ m \neq l}}^{N_{\mathrm{I}}} y_{m}}{\prod_{\substack{m=1 \\ m \neq n}}^{N_{\mathrm{I}}}(n-m)} .
$$

We implemented this in our numerical code, and checked it against known derivatives of test functions. The latter were combinations of sinusoidal and exponential functions, which have well-defined derivatives. Finally, we have

$$
\gamma^{\prime}\left(x_{l}\right)=\frac{1}{\Delta x} \sum_{m=a}^{b} C_{m} \gamma\left(x_{l+m}\right)
$$

with universal parameters $C_{m}$ for a fixed number $N_{\mathrm{I}}$ of integration points. 


\section{Bibliography}

[Balian 2007] R. Balian. From Microphysics to Macrophysics. Springer, 2007. URL http: //www.springer.com/fr/book/9783540454694.

[Basdevant and Dalibard 2002] J.-L. Basdevant and J. Dalibard. Quantum Mechanics. Springer, 2002. URL http://www.springer.com/us/book/ 9783540277064.

[Benthien et al. 2004] H. Benthien, F. Gebhard, and E. Jeckelmann. Spectral Function of the One-Dimensional Hubbard Model away from Half Filling. Phys. Rev. Lett., 92:256401, Jun 2004. doi: 10.1103/PhysRevLett.92.256401. URL http://link.aps.org/doi/10.1103/PhysRevLett.92.256401.

[Berges et al. 2004] J. Berges, S. Borsányi, and C. Wetterich. Prethermalization. Phys. Rev. Lett., 93:142002, Sep 2004. doi: 10.1103/PhysRevLett.93.142002. URL http://link.aps.org/doi/10.1103/PhysRevLett.93.142002.

[Bertini and Fagotti 2015] B. Bertini and M. Fagotti. Pre-relaxation in weakly interacting models. Journal of Statistical Mechanics: Theory and Experiment, 2015(7):P07012, 2015. URL http://stacks.iop.org/1742-5468/2015/i= $7 / \mathrm{a}=\mathrm{P} 07012$.

[Biebl and Kehrein 2016] F. R. A. Biebl and S. Kehrein. Thermalization rates in the one dimensional Hubbard model with next-to-nearest neighbor hopping. ArXiv e-prints, July 2016. URL http://arxiv.org/abs/1607.07115.

[Blanchard and Bruening 2003] P. Blanchard and E. Bruening. Mathematical Methods in Physics. Birkhäuser Basel, 2003. doi: 10.1007/978-1-4612-0049-9. URL http: //www.springer.com/us/book/9781461265894.

[Bloch et al. 2008] I. Bloch, J. Dalibard, and W. Zwerger. Many-body physics with ultracold gases. Rev. Mod. Phys., 80:885-964, Jul 2008. doi: 10.1103/RevModPhys.80.885. URL http://link.aps.org/doi/10.1103/ RevModPhys.80.885.

[Bloch et al. 2012] I. Bloch, J. Dalibard, and S. Nascimbene. Quantum simulations with ultracold quantum gases. Nat Phys, 8(4):267-276, 04 2012. URL http://dx.doi.org/10.1038/nphys2259.

[Blumenstein et al. 2011] C. Blumenstein, J. Schafer, S. Mietke, S. Meyer, A. Dollinger, M. Lochner, X. Y. Cui, L. Patthey, R. Matzdorf, and 
R. Claessen. Atomically controlled quantum chains hosting a TomonagaLuttinger liquid. Nat Phys, 7(10):776-780, 10 2011. URL http://dx.doi. org/10.1038/nphys2051.

[Claessen et al. 2002] R. Claessen, M. Sing, U. Schwingenschlögl, P. Blaha, M. Dressel, and C. S. Jacobsen. Spectroscopic Signatures of Spin-Charge Separation in the Quasi-One-Dimensional Organic Conductor TTF-TCNQ. Phys. Rev. Lett., 88:096402, Feb 2002. doi: 10.1103/PhysRevLett.88.096402. URL http://link.aps.org/doi/10.1103/PhysRevLett.88.096402.

[Cramer et al. 2008a] M. Cramer, C. M. Dawson, J. Eisert, and T. J. Osborne. Exact Relaxation in a Class of Nonequilibrium Quantum Lattice Systems. Phys. Rev. Lett., 100:030602, Jan 2008a. doi: 10.1103/PhysRevLett. 100.030602. URL http://link.aps.org/doi/10.1103/PhysRevLett.100. 030602.

[Cramer et al. 2008b] M. Cramer, A. Flesch, I. P. McCulloch, U. Schollwöck, and J. Eisert. Exploring Local Quantum Many-Body Relaxation by Atoms in Optical Superlattices. Phys. Rev. Lett., 101:063001, Aug 2008b. doi: 10. 1103/PhysRevLett.101.063001. URL http://link.aps.org/doi/10.1103/ PhysRevLett.101.063001.

[Dagotto 2003] E. Dagotto. Nanoscale Phase Separation and Colossal Magnetoresistance. Springer, 2003. URL http://www.springer.com/de/book/ 9783540432456

[Daley et al. 2004] A. J. Daley, C. Kollath, U. Schollwöck, and G. Vidal. Time-dependent density-matrix renormalization-group using adaptive effective Hilbert spaces. J. Stat. Mech., 2004(04):P04005, 2004. URL http: //stacks.iop.org/1742-5468/2004/i=04/a=P04005.

[Dalibard and Cohen-Tannoudji 1989] J. Dalibard and C. Cohen-Tannoudji. Laser cooling below the Doppler limit by polarization gradients: simple theoretical models. J. Opt. Soc. Am. B, 6(11):2023-2045, Nov 1989. doi: 10. 1364/JOSAB.6.002023. URL http://josab.osa.org/abstract.cfm?URI= josab-6-11-2023.

[Damascelli et al. 2003] A. Damascelli, Z. Hussain, and Z.-X. Shen. Angleresolved photoemission studies of the cuprate superconductors. Rev. Mod. Phys., 75:473-541, Apr 2003. doi: 10.1103/RevModPhys.75.473. URL http: //link.aps.org/doi/10.1103/RevModPhys.75.473.

[DePue et al. 1999] M. T. DePue, C. McCormick, S. L. Winoto, S. Oliver, and D. S. Weiss. Unity Occupation of Sites in a 3D Optical Lattice. Phys. Rev. Lett., 82:2262-2265, Mar 1999. doi: 10.1103/PhysRevLett.82.2262. URL http://link.aps.org/doi/10.1103/PhysRevLett.82.2262.

[Deutsch 1991] J. M. Deutsch. Quantum statistical mechanics in a closed system. Phys. Rev. A, 43:2046-2049, Feb 1991. doi: 10.1103/PhysRevA.43.2046. URL http://link.aps.org/doi/10.1103/PhysRevA.43.2046.

[Devereaux et al. 2004] T. P. Devereaux, T. Cuk, Z.-X. Shen, and N. Nagaosa. Anisotropic Electron-Phonon Interaction in the Cuprates. Phys. Rev. Lett., 
93:117004, Sep 2004. doi: 10.1103/PhysRevLett.93.117004. URL http:// link.aps.org/doi/10.1103/PhysRevLett.93.117004.

[Dolfen 2006] A. Dolfen. Massively parallel exact diagonalization of strongly correlated systems. $\mathrm{PhD}$ thesis, Institut für Festkörperforschung (IFF), Forschungszentrum Jülich, 2006. URL http://www.fz-juelich.de/cae/ servlet/contentblob/1040316/publicationFile/23068/Dolfen.pdf.

[Dressel 2003] M. Dressel. Spin-charge separation in quasi one-dimensional organic conductors. Naturwissenschaften, 90(8):337-344, 2003. ISSN 14321904. doi: 10.1007/s00114-003-0438-z. URL http://dx.doi.org/10.1007/ s00114-003-0438-z.

[Eckstein et al. 2009] M. Eckstein, M. Kollar, and P. Werner. Thermalization after an Interaction Quench in the Hubbard Model. Phys. Rev. Lett., 103: 056403, Jul 2009. doi: 10.1103/PhysRevLett.103.056403. URL http://Iink. aps.org/doi/10.1103/PhysRevLett.103.056403.

[Edmonds 1957] A. R. Edmonds. Angular Momentum in Quantum Mechanics. Princeton University Press, 1957.

[Egger and Gogolin 1998] R. Egger and A. Gogolin. Correlated transport and non-Fermi-liquid behavior in single-wall carbon nanotubes. The European Physical Journal B - Condensed Matter and Complex Systems, 3(3):281-300, 1998. ISSN 1434-6036. doi: 10.1007/s100510050315. URL http://dx.doi . org/10.1007/s100510050315.

[Egger and Gogolin 1997] R. Egger and A. O. Gogolin. Effective Low-Energy Theory for Correlated Carbon Nanotubes. Phys. Rev. Lett., 79:5082-5085, Dec 1997. doi: 10.1103/PhysRevLett.79.5082. URL http://link.aps.org/ doi/10.1103/PhysRevLett.79.5082.

[Einstein 1905] A. Einstein. Über einen die Erzeugung und Verwandlung des Lichtes betreffenden heuristischen Gesichtspunkt. Annalen der Physik, 322 (6):132-148, 1905. ISSN 1521-3889. doi: 10.1002/andp.19053220607. URL http://dx.doi.org/10.1002/andp.19053220607.

[Erdös et al. 2004] L. Erdös, M. Salmhofer, and H.-T. Yau. On the Quantum Boltzmann Equation. Journal of Statistical Physics, 116(1-4):367-380, 2004. ISSN 0022-4715. doi: 10.1023/B:JOSS.0000037224.56191.ed. URL http: //dx.doi.org/10.1023/B\%3AJOSS.0000037224.56191.ed.

[Essler et al. 2005] F. Essler, H. Frahm, F. Göhmann, A. Klümper, and V. Korepin. The One-Dimensional Hubbard Model. Cambridge University Press, 2005. URL http://ebooks . cambridge.org/ebook . jsf?bid=CB09780511534843.

[Essler et al. 2014] F. H. L. Essler, S. Kehrein, S. R. Manmana, and N. J. Robinson. Quench dynamics in a model with tuneable integrability breaking. Phys. Rev. B, 89:165104, Apr 2014. doi: 10.1103/PhysRevB.89.165104. URL http://link.aps.org/doi/10.1103/PhysRevB.89.165104.

[Fagotti 2014] M. Fagotti. On conservation laws, relaxation and pre-relaxation after a quantum quench. Journal of Statistical Mechanics: Theory and Experiment, 2014(3):P03016, 2014. URL http: //stacks . iop.org/1742-5468/ $2014 / i=3 / a=P 03016$. 
[Fano 1961] U. Fano. Effects of Configuration Interaction on Intensities and Phase Shifts. Phys. Rev., 124:1866-1878, Dec 1961. doi: 10.1103/PhysRev. 124.1866. URL http://link.aps.org/doi/10.1103/PhysRev.124.1866.

[Feshbach 1962] H. Feshbach. A unified theory of nuclear reactions. II. Annals of Physics, 19(2):287 - 313, 1962. ISSN 0003-4916. doi: http://dx. doi.org/10.1016/0003-4916(62)90221-X. URL http://www. sciencedirect . com/science/article/pii/000349166290221X.

[Fetter and Walecka 2003] A. Fetter and J. Walecka. Quantum Theory of Manyparticle Systems. Dover Books on Physics. Dover Publications, 2003. ISBN 9780486428277. URL https://books . google.de/books?id=0wekf1s83b0C.

[Friedrich 2006] H. Friedrich. Theoretical Atomic Physics. Springer, 2006.

[Fürst et al. 2012] M. L. R. Fürst, C. B. Mendl, and H. Spohn. Matrix-valued Boltzmann equation for the Hubbard chain. Phys. Rev. E, 86:031122, Sep 2012. doi: 10.1103/PhysRevE.86.031122. URL http://link.aps.org/doi/ 10.1103/PhysRevE.86.031122.

[Fürst et al. 2013a] M. L. R. Fürst, J. Lukkarinen, P. Mei, and H. Spohn. Derivation of a matrix-valued Boltzmann equation for the Hubbard model. Journal of Physics A: Mathematical and Theoretical, 46(48):485002, 2013a. URL http://stacks.iop.org/1751-8121/46/i=48/a=485002.

[Fürst et al. 2013b] M. L. R. Fürst, C. B. Mendl, and H. Spohn. Matrixvalued Boltzmann equation for the nonintegrable Hubbard chain. Phys. Rev. E, 88:012108, Jul 2013b. doi: 10.1103/PhysRevE.88.012108. URL http: //link.aps.org/doi/10.1103/PhysRevE.88.012108.

[Głazek and Wilson 1993] S. D. Głazek and K. G. Wilson. Renormalization of Hamiltonians. Phys. Rev. D, 48:5863-5872, Dec 1993. doi: 10.1103/ PhysRevD.48.5863. URL http://link.aps.org/doi/10.1103/PhysRevD. 48.5863 .

[Głazek and Wilson 1994] S. D. Głazek and K. G. Wilson. Perturbative renormalization group for Hamiltonians. Phys. Rev. D, 49:4214-4218, Apr 1994. doi: 10.1103/PhysRevD.49.4214. URL http://link.aps.org/doi/ 10.1103/PhysRevD. 49.4214.

[Goodenough 1963] J. B. Goodenough. Magnetism and the chemical bond. Interscience Publishers (Wiley), New York - London, 1963.

[Goodman 2009] T. S. Goodman. Strongly Interacting Fermions in Optical Lattices and Superlattices. PhD thesis, University of Michigan, 2009. URL http://hdl. handle.net/2027.42/62290.

[Gottfried and Yan 2003] K. Gottfried and T.-M. Yan. Quantum Mechanics: Fundamentals (Second Edition). Springer, 2003.

[Graf et al. 2011] J. Graf, C. Jozwiak, C. L. Smallwood, H. Eisaki, R. A. Kaindl, D.-H. Lee, and A. Lanzara. Nodal quasiparticle meltdown in ultrahighresolution pump-probe angle-resolved photoemission. Nat Phys, 7(10):805809, 10 2011. URL http://dx.doi.org/10.1038/nphys2027. 
[Greiner et al. 2002a] M. Greiner, O. Mandel, T. Esslinger, T. W. Hänsch, and I. Bloch. Quantum phase transition from a superfluid to a Mott insulator in a gas of ultracold atoms. Nature, 415(6867):39-44, 01 2002a. URL http: //dx.doi.org/10.1038/415039a.

[Greiner et al. 2002b] M. Greiner, O. Mandel, T. W. Hänsch, and I. Bloch. Collapse and revival of the matter wave field of a Bose-Einstein condensate. Nature, 419(6902):51-54, Sept. 2002b. ISSN 0028-0836. URL http://dx. doi.org/10.1038/nature00968.

[Grimm et al. 2000] R. Grimm, M. Weidemüller, and Y. B. Ovchinnikov. Optical Dipole Traps for Neutral Atoms. Advances In Atomic, Molecular, and Optical Physics, 42:95 - 170, 2000. ISSN 1049-250X. doi: http://dx.doi.org/10.1016/ S1049-250X(08)60186-X. URL http://www.sciencedirect.com/science/ article/pii/S1049250X0860186X.

[Gring et al. 2012] M. Gring, M. Kuhnert, T. Langen, T. Kitagawa, B. Rauer, M. Schreitl, I. Mazets, D. A. Smith, E. Demler, and J. Schmiedmayer. Relaxation and Prethermalization in an Isolated Quantum System. Science, 337 (6100):1318-1322, 2012. ISSN 0036-8075. doi: 10.1126/science.1224953. URL http://science. sciencemag. org/content/337/6100/1318.

[Gunnarsson and Schönhammer 1987] O. Gunnarsson and K. Schönhammer. Chapter 64 Many-body formulation of spectra of mixed valence systems. In High Energy Spectroscopy, volume 10 of Handbook on the Physics and Chemistry of Rare Earths, pages 103 - 163. Elsevier, 1987. doi: http://dx. doi.org/10.1016/S0168-1273(87)10006-2. URL http://www . sciencedirect . $\mathrm{com} / \mathrm{science/article/pii/S0168127387100062.}$

[Gutzwiller 1963] M. C. Gutzwiller. Effect of Correlation on the Ferromagnetism of Transition Metals. Phys. Rev. Lett., 10:159-162, Mar 1963. doi: 10.1103/PhysRevLett.10.159. URL http://link.aps.org/doi/10.1103/ PhysRevLett.10.159.

[Hamerla and Uhrig 2013] S. A. Hamerla and G. S. Uhrig. Dynamical transition in interaction quenches of the one-dimensional Hubbard model. Phys. Rev. B, 87:064304, Feb 2013. doi: 10.1103/PhysRevB.87.064304. URL http: //link.aps.org/doi/10.1103/PhysRevB.87.064304.

[Haug and Jauho 1996] H. Haug and A.-P. Jauho. Quantum Kinetics in Transport and Optics of Semiconductors. Springer, 1996. URL http: //www.springer.com/de/book/9783540735618.

[Hedin and Lundqvist 1970] L. Hedin and S. Lundqvist. Effects of ElectronElectron and Electron-Phonon Interactions on the One-Electron States of Solids. In D. T. Frederick Seitz and H. Ehrenreich, editors, Solid State Physics, volume 23, pages 1 - 181. Academic Press, 1970. doi: http://dx. doi.org/10.1016/S0081-1947(08)60615-3. URL http://www . sciencedirect . com/science/article/pii/S0081194708606153.

[Hertz 1887] H. Hertz. Ueber einen Einfluss des ultravioletten Lichtes auf die electrische Entladung. Annalen der Physik, 267(8):983-1000, 1887. ISSN 1521-3889. doi: 10.1002/andp.18872670827. URL http://dx.doi.org/10. 1002/andp. 18872670827. 
[Hofferberth et al. 2007] S. Hofferberth, I. Lesanovsky, B. Fischer, T. Schumm, and J. Schmiedmayer. Non-equilibrium coherence dynamics in onedimensional Bose gases. Nature, 449(7160):324-327, Sept. 2007. ISSN 00280836. URL http://dx.doi.org/10.1038/nature06149.

[Hubbard 1963] J. Hubbard. Electron Correlations in Narrow Energy Bands. Proceedings of the Royal Society of London A: Mathematical, Physical and Engineering Sciences, 276(1365):238-257, 1963. ISSN 0080-4630. doi: 10. 1098/rspa.1963.0204. URL http://rspa.royalsocietypublishing.org/ content/276/1365/238.

[Hubbard 1964a] J. Hubbard. Electron Correlations in Narrow Energy Bands. II. The Degenerate Band Case. Proceedings of the Royal Society of London A: Mathematical, Physical and Engineering Sciences, 277(1369):237259, 1964a. ISSN 0080-4630. doi: 10.1098/rspa.1964.0019. URL http: //rspa.royalsocietypublishing. org/content/277/1369/237.

[Hubbard 1964b] J. Hubbard. Electron Correlations in Narrow Energy Bands. III. An Improved Solution. Proceedings of the Royal Society of London A: Mathematical, Physical and Engineering Sciences, 281(1386):401-419, 1964b. ISSN 0080-4630. doi: 10.1098/rspa.1964.0190. URL http://rspa. royalsocietypublishing.org/content/281/1386/401.

[Ishii et al. 2003] H. Ishii, H. Kataura, H. Shiozawa, H. Yoshioka, H. Otsubo, Y. Takayama, T. Miyahara, S. Suzuki, Y. Achiba, M. Nakatake, T. Narimura, M. Higashiguchi, K. Shimada, H. Namatame, and M. Taniguchi. Direct observation of Tomonaga-Luttinger-liquid state in carbon nanotubes at low temperatures. Nature, 426(6966):540-544, 12 2003. URL http: //dx.doi.org/10.1038/nature02074.

[Jaynes 1957a] E. T. Jaynes. Information Theory and Statistical Mechanics. Phys. Rev., 106:620-630, May 1957a. doi: 10.1103/PhysRev.106.620. URL http://link.aps.org/doi/10.1103/PhysRev.106.620.

[Jaynes 1957b] E. T. Jaynes. Information Theory and Statistical Mechanics. II. Phys. Rev., 108:171-190, Oct 1957b. doi: 10.1103/PhysRev.108.171. URL http://link.aps.org/doi/10.1103/PhysRev.108.171.

[Johnston 1984] D. C. Johnston. Thermodynamics of Charge-Density Waves in Quasi One-Dimensional Conductors. Phys. Rev. Lett., 52:2049-2052, Jun 1984. doi: 10.1103/PhysRevLett.52.2049. URL http://link.aps.org/doi/ 10.1103/PhysRevLett.52.2049.

[Jones et al. 2006] K. M. Jones, E. Tiesinga, P. D. Lett, and P. S. Julienne. Ultracold photoassociation spectroscopy: Long-range molecules and atomic scattering. Rev. Mod. Phys., 78:483-535, May 2006. doi: 10.1103/RevModPhys. 78.483. URL http://link.aps.org/doi/10.1103/RevModPhys.78.483.

[Jérome and Schulz 1982] D. Jérome and H. Schulz. Organic conductors and superconductors. Advances in Physics, 31(4):299-490, 1982. doi: 10.1080/00018738200101398. URL http://dx.doi.org/10.1080/ 00018738200101398 . 
[Jérome, D. et al. 1980] Jérome, D., Mazaud, A., Ribault, M., and Bechgaard, K. Superconductivity in a synthetic organic conductor (TMTSF)2PF 6. J. Physique Lett., 41(4):95-98, 1980. doi: 10.1051/jphyslet:0198000410409500. URL http://dx.doi.org/10.1051/jphyslet:0198000410409500.

[Kabanov and Alexandrov 2008] V. V. Kabanov and A. S. Alexandrov. Electron relaxation in metals: Theory and exact analytical solutions. Phys. Rev. B, 78: 174514, Nov 2008. doi: 10.1103/PhysRevB.78.174514. URL http://link . aps.org/doi/10.1103/PhysRevB.78.174514.

[Kaldis et al. 1989] E. Kaldis, P. Fischer, A. Hewat, E. Hewat, J. Karpinski, and S. Rusiecki. Low temperature anomalies and pressure effects on the structure and Tc of the superconductor $\mathrm{YBa} 2 \mathrm{Cu} 4 \mathrm{O} 8(\mathrm{Tc}=80 \mathrm{~K})$. Physica $C$ : Superconductivity, 159(5):668 - 680, 1989. ISSN 0921-4534. doi: http://dx. doi.org/10.1016/0921-4534(89)91303-8. URL http://www. sciencedirect. com/science/article/pii/0921453489913038.

[Kamenev and Levchenko 2009] A. Kamenev and A. Levchenko. Keldysh technique and non-linear $\sigma$-model: basic principles and applications. Advances in Physics, 58(3):197-319, 2009. doi: 10.1080/00018730902850504. URL http://dx.doi.org/10.1080/00018730902850504.

[Kanamori 1963] J. Kanamori. Electron Correlation and Ferromagnetism of Transition Metals. Progress of Theoretical Physics, 30(3):275-289, 1963. doi: 10.1143/PTP.30.275. URL http://ptp.oxfordjournals.org/content/30/ 3/275. abstract.

[Kane et al. 1997] C. Kane, L. Balents, and M. P. A. Fisher. Coulomb Interactions and Mesoscopic Effects in Carbon Nanotubes. Phys. Rev. Lett., 79:5086-5089, Dec 1997. doi: 10.1103/PhysRevLett.79.5086. URL http: //link.aps.org/doi/10.1103/PhysRevLett.79.5086.

[Karpinski et al. 1988] J. Karpinski, E. Kaldis, E. Jilek, S. Rusiecki, and B. Bucher. Bulk synthesis of the $81-\mathrm{K}$ superconductor $\mathrm{YBa} 2 \mathrm{Cu} 4 \mathrm{O} 8$ at high oxygen pressure. Nature, 336(6200):660-662, 12 1988. URL http: //dx.doi.org/10.1038/336660a0.

[Kehrein 2006] S. Kehrein. The Flow Equation Approach to Many-Particle Systems. Springer Science \& Business Media, 2006. URL http://www . springer. com/us/book/9783540340676.

[Keren et al. 1993] A. Keren, L. P. Le, G. M. Luke, B. J. Sternlieb, W. D. Wu, Y. J. Uemura, S. Tajima, and S. Uchida. Muon-spin-rotation measurements in infinite-layer and infinite-chain cuprate antiferromagnets: $\mathrm{Ca}_{0.86} \mathrm{Sr}_{0.14} \mathrm{CuO}_{2}$ and $\mathrm{Sr}_{2} \mathrm{CuO}_{3}$. Phys. Rev. B, 48:12926-12935, Nov 1993. doi: 10.1103/ PhysRevB.48.12926. URL http://link.aps.org/doi/10.1103/PhysRevB. 48.12926.

[Kim et al. 2006] B. J. Kim, H. Koh, E. Rotenberg, S. J. Oh, H. Eisaki, N. Motoyama, S. Uchida, T. Tohyama, S. Maekawa, Z. X. Shen, and C. Kim. Distinct spinon and holon dispersions in photoemission spectral functions from one-dimensional SrCuO2. Nat Phys, 2(6):397-401, 06 2006. URL http://dx.doi.org/10.1038/nphys316. 
[Kim et al. 1996] C. Kim, A. Y. Matsuura, Z.-X. Shen, N. Motoyama, H. Eisaki, S. Uchida, T. Tohyama, and S. Maekawa. Observation of Spin-Charge Separation in One-Dimensional $\mathrm{SrCuO}_{2}$. Phys. Rev. Lett., 77:4054-4057, Nov 1996. doi: 10.1103/PhysRevLett.77.4054. URL http://link.aps.org/doi/ 10.1103/PhysRevLett.77.4054.

[Kinoshita et al. 2006] T. Kinoshita, T. Wenger, and D. S. Weiss. A quantum Newton's cradle. Nature, 440(7086):900-903, Apr. 2006. ISSN 0028-0836. URL http://dx.doi.org/10.1038/nature04693.

[Köhler et al. 2016] T. Köhler, S. Rajpurohit, O. Schumann, F. R. A. Biebl, M. Sotoudeh, S. C. Kramer, P. E. Blöchl, S. Kehrein, and S. R. Manmana. Photoexcitations in a 1D manganite model: From quasiclassical light absorption to quasiparticle relaxations. ArXiv e-prints, Oct. 2016. URL https://arxiv.org/abs/1610.07246.

[Koitzsch et al. 2006] A. Koitzsch, S. V. Borisenko, J. Geck, V. B. Zabolotnyy, M. Knupfer, J. Fink, P. Ribeiro, B. Büchner, and R. Follath. Current spinonholon description of the one-dimensional charge-transfer insulator $\mathrm{SrCuO}_{2}$ : Angle-resolved photoemission measurements. Phys. Rev. B, 73:201101, May 2006. doi: 10.1103/PhysRevB.73.201101. URL http://link.aps.org/doi/ 10.1103/PhysRevB.73.201101.

[Kojima et al. 1997] K. M. Kojima, Y. Fudamoto, M. Larkin, G. M. Luke, J. Merrin, B. Nachumi, Y. J. Uemura, N. Motoyama, H. Eisaki, S. Uchida, K. Yamada, Y. Endoh, S. Hosoya, B. J. Sternlieb, and G. Shirane. Reduction of Ordered Moment and Néel Temperature of Quasi-One-Dimensional Antiferromagnets $\mathrm{Sr}_{2} \mathrm{CuO}_{3}$ and $\mathrm{Ca}_{2} \mathrm{CuO}_{3}$. Phys. Rev. Lett., 78:1787-1790, Mar 1997. doi: 10.1103/PhysRevLett.78.1787. URL http://link.aps.org/doi/ 10.1103/PhysRevLett.78.1787.

[Kollath et al. 2007] C. Kollath, A. M. Läuchli, and E. Altman. Quench Dynamics and Nonequilibrium Phase Diagram of the Bose-Hubbard Model. Phys. Rev. Lett., 98:180601, Apr 2007. doi: 10.1103/PhysRevLett.98.180601. URL http://link.aps.org/doi/10.1103/PhysRevLett.98.180601.

[Kolobov and Tominaga 2016] A. V. Kolobov and J. Tominaga. TwoDimensional Transition-Metal Dichalcogenides. Springer, 2016. URL http: //www. springer.com/in/book/9783319314495.

[Kondo et al. 2010] T. Kondo, R. Khasanov, J. Karpinski, S. M. Kazakov, N. D. Zhigadlo, Z. Bukowski, M. Shi, A. Bendounan, Y. Sassa, J. Chang, S. Pailhés, J. Mesot, J. Schmalian, H. Keller, and A. Kaminski. Anomalies in the Fermi Surface and Band Dispersion of Quasi-One-Dimensional $\mathrm{CuO}$ Chains in the High-Temperature Superconductor $\mathrm{YBa}_{2} \mathrm{Cu}_{4} \mathrm{O}_{8}$. Phys. Rev. Lett., 105:267003, Dec 2010. doi: 10.1103/PhysRevLett.105.267003. URL http://link.aps.org/doi/10.1103/PhysRevLett.105.267003.

[Landau et al. 2007] R. H. Landau, M. J. Paez, and C. C. Bordelanu. Computational Physics. Wiley, 2007. 
[Langen et al. 2015] T. Langen, S. Erne, R. Geiger, B. Rauer, T. Schweigler, M. Kuhnert, W. Rohringer, I. E. Mazets, T. Gasenzer, and J. Schmiedmayer. Experimental observation of a generalized Gibbs ensemble. Science, 348(6231):207-211, 2015. doi: 10.1126/science.1257026. URL http: //www. sciencemag. org/content/348/6231/207. abstract.

[Letokhov and Minogin 1981] V. Letokhov and V. Minogin. Laser radiation pressure on free atoms. Physics Reports, 73(1):1 - 65, 1981. ISSN 03701573. doi: http://dx.doi.org/10.1016/0370-1573(81)90116-2. URL http:// www. sciencedirect.com/science/article/pii/0370157381901162.

[Levin et al. 2012] K. Levin, A. L. Fetter, and D. M. Stamper-Kurn. Ultracold Bosonic and Fermionic Gases. Elsevier, 2012.

[Lewenstein et al. 2012] M. Lewenstein, A. Sanpera, and V. Ahufinger. Ultracold Atoms in Optical Lattices. Oxford University Press, 2012.

[Li 2008] H. Li. Synthesis of CMR manganites and ordering phenomena in complex transition metal oxides. Forschungszentrum Jülich GmbH, 2008. URL http://hdl.handle.net/2128/3141.

[Liu et al. 2013] H. Y. Liu, I. Gierz, J. C. Petersen, S. Kaiser, A. Simoncig, A. L. Cavalieri, C. Cacho, I. C. E. Turcu, E. Springate, F. Frassetto, L. Poletto, S. S. Dhesi, Z.-A. Xu, T. Cuk, R. Merlin, and A. Cavalleri. Possible observation of parametrically amplified coherent phasons in $\mathrm{K}_{0.3} \mathrm{MoO}_{3}$ using time-resolved extreme-ultraviolet angle-resolved photoemission spectroscopy. Phys. Rev. B, 88:045104, Jul 2013. doi: 10.1103/PhysRevB.88.045104. URL http://link. aps.org/doi/10.1103/PhysRevB.88.045104.

[Mahan 1990] G. D. Mahan. Many-Particle Physics. Plenum Press, 1990.

[Manmana et al. 2005] S. R. Manmana, A. Muramatsu, and R. M. Noack. Time evolution of one-dimensional Quantum Many Body Systems. AIP Conference Proceedings, 789(1):269-278, 2005. doi: http://dx.doi.org/10.1063/ 1.2080353. URL http://scitation.aip.org/content/aip/proceeding/ aipcp/10.1063/1.2080353.

[Manmana et al. 2007] S. R. Manmana, S. Wessel, R. M. Noack, and A. Muramatsu. Strongly Correlated Fermions after a Quantum Quench. Phys. Rev. Lett., 98:210405, May 2007. doi: 10.1103/PhysRevLett.98.210405. URL http://link.aps.org/doi/10.1103/PhysRevLett.98.210405.

[Mathur and Singh 2009] V. S. Mathur and S. Singh. Concepts in Quantum Mechanics. CRC Press, 2009.

[Moeckel and Kehrein 2008] M. Moeckel and S. Kehrein. Interaction Quench in the Hubbard Model. Phys. Rev. Lett., 100:175702, May 2008. doi: 10. 1103/PhysRevLett.100.175702. URL http://link.aps.org/doi/10.1103/ PhysRevLett.100.175702.

[Motoyama et al. 1996] N. Motoyama, H. Eisaki, and S. Uchida. Magnetic Susceptibility of Ideal Spin $1 / 2$ Heisenberg Antiferromagnetic Chain Systems, $\mathrm{Sr}_{2} \mathrm{CuO}_{3}$ and $\mathrm{SrCuO}_{2}$. Phys. Rev. Lett., 76:3212-3215, Apr 1996. doi: 10.1103/PhysRevLett.76.3212. URL http://link.aps.org/doi/10.1103/ PhysRevLett.76.3212. 
[Mueller and Rethfeld 2013] B. Y. Mueller and B. Rethfeld. Relaxation dynamics in laser-excited metals under nonequilibrium conditions. Phys. Rev. B, 87: 035139, Jan 2013. doi: 10.1103/PhysRevB.87.035139. URL http://link. aps.org/doi/10.1103/PhysRevB.87.035139.

[Ni et al. 2012] H. Ni, Z. Yue, K. Zhao, W. Xiang, S. Zhao, A. Wang, Y.-C. Kong, and H.-K. Wong. Magnetical and electrical tuning of transient photovoltaic effects in manganite-based heterojunctions. Opt. Express, 20(S3):A406-A411, May 2012. doi: 10.1364/OE.20.00A406. URL http://www.opticsexpress . org/abstract . cfm?URI=oe-20-103-A406.

[Perfetti et al. 2002] L. Perfetti, S. Mitrovic, G. Margaritondo, M. Grioni, L. Forró, L. Degiorgi, and H. Höchst. Mobile small polarons and the Peierls transition in the quasi-one-dimensional conductor $\mathrm{K}_{0.3} \mathrm{MoO}_{3}$. Phys. Rev. B, 66:075107, Aug 2002. doi: 10.1103/PhysRevB.66.075107. URL http://link.aps.org/doi/10.1103/PhysRevB.66.075107.

[Perfetti et al. 2007] L. Perfetti, P. A. Loukakos, M. Lisowski, U. Bovensiepen, H. Eisaki, and M. Wolf. Ultrafast Electron Relaxation in Superconducting $\mathrm{Bi}_{2} \mathrm{Sr}_{2} \mathrm{CaCu}_{2} \mathrm{O}_{8+\delta}$ by Time-Resolved Photoelectron Spectroscopy. Phys. Rev. Lett., 99:197001, Nov 2007. doi: 10.1103/PhysRevLett.99.197001. URL http: //link.aps.org/doi/10.1103/PhysRevLett.99.197001.

[Pethick and Smith 2008] C. J. Pethick and H. Smith. Bose-Einstein Condensation in Dilute Gases. Cambridge University Press, 2008.

[Phillips 1998] W. D. Phillips. Nobel Lecture: Laser cooling and trapping of neutral atoms. Rev. Mod. Phys., 70:721-741, Jul 1998. doi: 10.1103/RevModPhys.70.721. URL http://link.aps.org/doi/10.1103/ RevModPhys.70.721.

[Polkovnikov et al. 2011] A. Polkovnikov, K. Sengupta, A. Silva, and M. Vengalattore. Colloquium : Nonequilibrium dynamics of closed interacting quantum systems. Rev. Mod. Phys., 83:863-883, Aug 2011. doi: 10.1103/RevModPhys.83.863. URL http://link.aps.org/doi/10.1103/ RevModPhys. 83.863.

[Popescu et al. 2006] S. Popescu, A. J. Short, and A. Winter. Entanglement and the foundations of statistical mechanics. Nat Phys, 2(11):754-758, Nov. 2006. ISSN 1745-2473. URL http://dx.doi.org/10.1038/nphys444.

[Rajpurohit and Blöchl 2016] S. Rajpurohit and P. Blöchl. Private communication, Mar. 2016.

[Rammer and Smith 1986] J. Rammer and H. Smith. Quantum field-theoretical methods in transport theory of metals. Rev. Mod. Phys., 58:323-359, Apr 1986. doi: 10.1103/RevModPhys.58.323. URL http://link.aps.org/doi/ 10.1103/RevModPhys.58.323.

[Rettig et al. 2012] L. Rettig, R. Cortés, S. Thirupathaiah, P. Gegenwart, H. S. Jeevan, M. Wolf, J. Fink, and U. Bovensiepen. Ultrafast MomentumDependent Response of Electrons in Antiferromagnetic $\mathrm{EuFe}_{2} \mathrm{As}_{2}$ Driven by Optical Excitation. Phys. Rev. Lett., 108:097002, Feb 2012. doi: 10. 
1103/PhysRevLett.108.097002. URL http://link.aps.org/doi/10.1103/ PhysRevLett.108.097002.

[Rigol 2009] M. Rigol. Breakdown of Thermalization in Finite One-Dimensional Systems. Phys. Rev. Lett., 103:100403, Sep 2009. doi: 10.1103/PhysRevLett. 103.100403. URL http://link.aps.org/doi/10.1103/PhysRevLett.103. 100403.

[Rigol and Srednicki 2012] M. Rigol and M. Srednicki. Alternatives to Eigenstate Thermalization. Phys. Rev. Lett., 108:110601, Mar 2012. doi: 10. 1103/PhysRevLett.108.110601. URL http://link.aps.org/doi/10.1103/ PhysRevLett.108.110601.

[Rigol et al. 2007] M. Rigol, V. Dunjko, V. Yurovsky, and M. Olshanii. Relaxation in a Completely Integrable Many-Body Quantum System: An $A b$ Initio Study of the Dynamics of the Highly Excited States of 1D Lattice Hard-Core Bosons. Phys. Rev. Lett., 98:050405, Feb 2007. doi: 10. 1103/PhysRevLett.98.050405. URL http://link.aps.org/doi/10.1103/ PhysRevLett.98.050405.

[Rigol et al. 2008] M. Rigol, V. Dunjko, and M. Olshanii. Thermalization and its mechanism for generic isolated quantum systems. Nature, 452(7189): 854-858, Apr. 2008. ISSN 0028-0836. URL http://dx.doi.org/10.1038/ nature06838.

[Rohwer et al. 2011] T. Rohwer, S. Hellmann, M. Wiesenmayer, C. Sohrt, A. Stange, B. Slomski, A. Carr, Y. Liu, L. M. Avila, M. Kallane, S. Mathias, L. Kipp, K. Rossnagel, and M. Bauer. Collapse of long-range charge order tracked by time-resolved photoemission at high momenta. Nature, 471(7339): 490-493, 03 2011. URL http://dx.doi.org/10.1038/nature09829.

[Roux 2009] G. Roux. Quenches in quantum many-body systems: Onedimensional Bose-Hubbard model reexamined. Phys. Rev. A, 79:021608, Feb 2009. doi: 10.1103/PhysRevA.79.021608. URL http://link.aps.org/doi/ 10.1103/PhysRevA.79.021608.

[Sakurai 1994] J. J. Sakurai. Modern Quantum Mechanics, revised edition. Addison-Wesley, 1994.

[Santos and Rigol 2010] L. F. Santos and M. Rigol. Onset of quantum chaos in one-dimensional bosonic and fermionic systems and its relation to thermalization. Phys. Rev. E, 81:036206, Mar 2010. doi: 10.1103/PhysRevE.81.036206. URL http://link.aps.org/doi/10.1103/PhysRevE.81.036206.

[Saucke et al. 2012] G. Saucke, J. Norpoth, C. Jooss, D. Su, and Y. Zhu. Polaron absorption for photovoltaic energy conversion in a manganite-titanate $p n$ heterojunction. Phys. Rev. B, 85:165315, Apr 2012. doi: 10.1103/PhysRevB.85. 165315. URL http://link.aps.org/doi/10.1103/PhysRevB.85.165315.

[Schmitt et al. 2008] F. Schmitt, P. S. Kirchmann, U. Bovensiepen, R. G. Moore, L. Rettig, M. Krenz, J.-H. Chu, N. Ru, L. Perfetti, D. H. Lu, M. Wolf, I. R. Fisher, and Z.-X. Shen. Transient Electronic Structure and Melting of a Charge Density Wave in TbTe3. Science, 321(5896): 
1649-1652, 2008. ISSN 0036-8075. doi: 10.1126/science.1160778. URL http://science.sciencemag.org/content/321/5896/1649.

[Schollwöck 2005] U. Schollwöck. The density-matrix renormalization group. Rev. Mod. Phys., 77:259-315, Apr 2005. doi: 10.1103/RevModPhys.77.259. URL http://link .aps .org/doi/10.1103/RevModPhys .77.259.

[Schollwöck 2011] U. Schollwöck. The density-matrix renormalization group in the age of matrix product states. Annals of Physics, 326(1):96 192, 2011. ISSN 0003-4916. doi: http://dx.doi.org/10.1016/j.aop.2010. 09.012. URL http://www.sciencedirect.com/science/article/pii/ S0003491610001752. January 2011 Special Issue.

[Schreiber et al. 2015] M. Schreiber, S. S. Hodgman, P. Bordia, H. P. Lüschen, M. H. Fischer, R. Vosk, E. Altman, U. Schneider, and I. Bloch. Observation of many-body localization of interacting fermions in a quasirandom optical lattice. Science, 349(6250):842-845, 2015. ISSN 0036-8075. doi: 10.1126/science. aaa7432. URL http://science. sciencemag.org/content/349/6250/842.

[Schrödinger 1926a] E. Schrödinger. Quantisierung als Eigenwertproblem. Annalen der Physik, 384(4):361-376, 1926a. ISSN 1521-3889. doi: 10.1002/andp. 19263840404. URL http://dx.doi.org/10.1002/andp.19263840404.

[Schrödinger 1926b] E. Schrödinger. Quantisierung als Eigenwertproblem. Annalen der Physik, 384(6):489-527, 1926b. ISSN 1521-3889. doi: 10.1002/ andp.19263840602. URL http://dx.doi.org/10.1002/andp. 19263840602.

[Schrödinger 1926c] E. Schrödinger. Quantisierung als Eigenwertproblem. Annalen der Physik, 385(13):437-490, 1926c. ISSN 1521-3889. doi: 10.1002/ andp.19263851302. URL http://dx.doi.org/10.1002/andp.19263851302.

[Schrödinger 1926d] E. Schrödinger. Quantisierung als Eigenwertproblem. Annalen der Physik, 386(18):109-139, 1926d. ISSN 1521-3889. doi: 10.1002/ andp.19263861802. URL http://dx.doi.org/10.1002/andp.19263861802.

[Seabra et al. 2014] L. Seabra, F. H. L. Essler, F. Pollmann, I. Schneider, and T. Veness. Real-time dynamics in the one-dimensional Hubbard model. Phys. Rev. B, 90:245127, Dec 2014. doi: 10.1103/PhysRevB.90.245127. URL http: //link.aps.org/doi/10.1103/PhysRevB.90.245127.

[Sesko et al. 1989] D. Sesko, T. Walker, C. Monroe, A. Gallagher, and C. Wieman. Collisional losses from a light-force atom trap. Phys. Rev. Lett., 63:961-964, Aug 1989. doi: 10.1103/PhysRevLett.63.961. URL http: //link.aps.org/doi/10.1103/PhysRevLett.63.961.

[Sing et al. 2003] M. Sing, U. Schwingenschlögl, R. Claessen, P. Blaha, J. M. P. Carmelo, L. M. Martelo, P. D. Sacramento, M. Dressel, and C. S. Jacobsen. Electronic structure of the quasi-one-dimensional organic conductor TTFTCNQ. Phys. Rev. B, 68:125111, Sep 2003. doi: 10.1103/PhysRevB.68. 125111. URL http://link.aps.org/doi/10.1103/PhysRevB.68.125111.

[Smallwood et al. 2012] C. L. Smallwood, J. P. Hinton, C. Jozwiak, W. Zhang, J. D. Koralek, H. Eisaki, D.-H. Lee, J. Orenstein, and A. Lanzara. Tracking Cooper Pairs in a Cuprate Superconductor by Ultrafast Angle-Resolved 
Photoemission. Science, 336(6085):1137-1139, 2012. ISSN 0036-8075. doi: 10.1126/science.1217423. URL http://science.sciencemag.org/content/ 336/6085/1137.

[Spohn 2014] H. Spohn. Private communication, July 2014.

[Srednicki 1994] M. Srednicki. Chaos and quantum thermalization. Phys. Rev. E, 50:888-901, Aug 1994. doi: 10.1103/PhysRevE.50.888. URL http:// link.aps.org/doi/10.1103/PhysRevE.50.888.

[Stenholm 1986] S. Stenholm. The semiclassical theory of laser cooling. Rev. Mod. Phys., 58:699-739, Jul 1986. doi: 10.1103/RevModPhys.58.699. URL http://link.aps.org/doi/10.1103/RevModPhys.58.699.

[Stoof et al. 2009] H. T. Stoof, K. B. Gubbels, and D. B. Dickerscheid. Ultracold Quantum Fields. Springer, 2009.

[Stwalley 1976] W. C. Stwalley. Stability of Spin-Aligned Hydrogen at Low Temperatures and High Magnetic Fields: New Field-Dependent Scattering Resonances and Predissociations. Phys. Rev. Lett., 37:1628-1631, Dec 1976. doi: 10.1103/PhysRevLett.37.1628. URL http://link.aps.org/doi/10.1103/ PhysRevLett. 37.1628.

[Travaglini et al. 1981] G. Travaglini, P. Wachter, J. Marcus, and C. Schlenker. The blue bronze $\mathrm{K}_{0.3} \mathrm{MoO}_{3}$ : A new one-dimensional conductor. Solid State Communications, 37(7):599 - 603, 1981. ISSN 0038-1098. doi: http://dx.doi. org/10.1016/0038-1098(81)90143-5. URL http://www.sciencedirect.com/ science/article/pii/0038109881901435.

[Wegner 1994] F. Wegner. Flow equations for Hamiltonians. Ann. Physik, 3: 77, 1994. doi: 10.1002/andp.19945060203. URL https://dx.doi.org/10. 1002/andp. 19945060203.

[Weiner 2003] J. Weiner. Cold and Ultracold Collisions in Quantum Microscopic and Mesoscopic Systems. Cambridge University Press, 2003.

[White 1992] S. R. White. Density matrix formulation for quantum renormalization groups. Phys. Rev. Lett., 69:2863-2866, Nov 1992. doi: 10.1103/PhysRevLett.69.2863. URL http://link.aps.org/doi/10.1103/ PhysRevLett.69.2863.

[White 1993] S. R. White. Density-matrix algorithms for quantum renormalization groups. Phys. Rev. B, 48:10345-10356, Oct 1993. doi: 10.1103/ PhysRevB.48.10345. URL http://link.aps.org/doi/10.1103/PhysRevB . 48.10345.

[Wineland and Itano 1979] D. J. Wineland and W. M. Itano. Laser cooling of atoms. Phys. Rev. A, 20:1521-1540, Oct 1979. doi: 10.1103/PhysRevA.20. 1521. URL http://link.aps.org/doi/10.1103/PhysRevA.20.1521.

[Wollan and Koehler 1955] E. O. Wollan and W. C. Koehler. Neutron Diffraction Study of the Magnetic Properties of the Series of Perovskite-Type Compounds $[(1-x) \mathrm{La}, x \mathrm{Ca}] \mathrm{MnO}_{3}$. Phys. Rev., 100:545-563, Oct 1955. doi: 10.1103/ PhysRev.100.545. URL http://link. aps.org/doi/10.1103/PhysRev. 100. 545. 
[Yunoki et al. 2000] S. Yunoki, T. Hotta, and E. Dagotto. Ferromagnetic, $A$-Type, and Charge-Ordered CE-Type States in Doped Manganites Using Jahn-Teller Phonons. Phys. Rev. Lett., 84:3714-3717, Apr 2000. doi: 10.1103/PhysRevLett.84.3714. URL http://link.aps.org/doi/10.1103/ PhysRevLett.84.3714.

[Zhou and Goodenough 1996] J. S. Zhou and J. B. Goodenough. Pressure dependence of thermopower in $\mathrm{YBa}_{2} \mathrm{Cu}_{3} \mathrm{O}_{6.96}$ and $\mathrm{YBa}_{2} \mathrm{Cu}_{4} \mathrm{O}_{8}$. Phys. Rev. B, 53:R11976-R11979, May 1996. doi: 10.1103/PhysRevB.53.R11976. URL http://link.aps.org/doi/10.1103/PhysRevB.53.R11976.

[Zwick et al. 1997] F. Zwick, S. Brown, G. Margaritondo, C. Merlic, M. Onellion, J. Voit, and M. Grioni. Absence of Quasiparticles in the Photoemission Spectra of Quasi-one-dimensional Bechgaard Salts. Phys. Rev. Lett., 79:3982-3985, Nov 1997. doi: 10.1103/PhysRevLett.79.3982. URL http: //link.aps.org/doi/10.1103/PhysRevLett.79.3982. 


\section{Curriculum Vitae}

\section{Personal Details}

$\begin{array}{ll}\text { Surname } & \text { Biebl } \\ \text { First names } & \text { Fabian Ralf Anton } \\ \text { Date of birth } & 12^{\text {th }} \text { of March 1987 } \\ \text { Place of birth } & \text { Munich, Germany } \\ \text { Nationality } & \text { German }\end{array}$

\section{Scientific career / PHD / Studies}

11.2016 - today Post-doctoral position

School of Computer Science \& Informatics

University of Cardiff

Group of Prof. David Walker

(High Performance Computing)

Finite elements programming using CUDA for Magnetic Induction Tomography

$12.2012-12.2016$

Doctorate

Institute for theoretical condensed matter physics

University of Göttingen, Germany

Group of Prof. Dr. Stefan Kehrein

(Condensed matter theory)

10.2007 - 11.2012 Diploma degree program in general physics

Technical University of Munich, Germany

Final grade: 1.4 ("very good")

Specializations:

Computational physics

Advanced theoretical physics

Diploma thesis:

Interference contrast in one-dimensional Bose-gases

Group of Wilhelm Zwerger

(Condensed matter \& many-body theory)

\section{School / Civil service}

09.2006 - 06.2007 Transport services for elderly citizens at the ArbeiterSamariter-Bund in Munich

$09.1997-06.2006 \quad$ Advanced secondary school in Munich

Abitur (graduation examination): 2.2 ("good") 


\section{Talks}

17.03.2015 "Thermalization rates in a 1d Fermi-Hubbard model with slightly broken integrability for various fillings"

DPG Spring Meeting, Berlin, Germany

03.04.2014 "Thermalization timescales in a 1d Hubbard model with slightly broken integrability"

DPG Spring Meeting, Dresden, Germany

07.03.2014 "Thermalization timescales in a 1d Hubbard model with slightly broken integrability"

APS March Meeting, Denver, Colorado, USA

\section{Publications}

23.10 .2016

Photoexcitations in a 1D manganite model: From quasiclassical light absorption to quasiparticle relaxations https://arxiv.org/abs/1610.07246

25.07.2016 Thermalization rates in the one dimensional Hubbard model with next-to-nearest neighbor hopping

https://arxiv.org/abs/1607.07115 